OAK RIDGE

ORNL/TM-2008/131

NATIONAL LABORATORY

MANAGED BY UT-BATTELLE FOR

THE DEPARTMENT OF ENERGY

\title{
Reliability of Current Biokinetic and Dosimetric Models for Radionuclides: A Pilot Study
}

August 2008

Prepared by

R. W. Leggett ${ }^{a}$

K. F. Eckerman ${ }^{a}$

R. A. Meck ${ }^{\mathrm{b}}$

aak Ridge National Laboratory

Oak Ridge, Tennessee 37831

${ }^{b}$ Office of Nuclear Regulatory Research

U.S. Nuclear Regulatory Commission 


\section{DOCUMENT AVAILABILITY}

Reports produced after January 1, 1996, are generally available free via the U.S. Department of Energy (DOE) Information Bridge:

Web site: http://www.osti.gov/bridge

Reports produced before January 1, 1996, may be purchased by members of the public from the following source:

National Technical Information Service

5285 Port Royal Road

Springfield, VA 22161

Telephone: 703-605-6000 (1-800-553-6847)

TDD: 703-487-4639

Fax: 703-605-6900

E-mail: info@ntis.fedworld.gov

Web site: http://www.ntis.gov/support/ordernowabout.htm

Reports are available to DOE employees, DOE contractors, Energy Technology Data Exchange (ETDE) representatives, and International Nuclear Information System (INIS) representatives from the following source:

Office of Scientific and Technical Information

P.O. Box 62

Oak Ridge, TN 37831

Telephone: 865-576-8401

Fax: 865-576-5728

E-mail: reports@adonis.osti.gov

Web site: http://www.osti.gov/contact.html

This report was prepared as an account of work sponsored by an agency of the United States Government. Neither the United States government nor any agency thereof, nor any of their employees, makes any warranty, express or implied, or assumes any legal liability or responsibility for the accuracy, completeness, or usefulness of any information, apparatus, product, or process disclosed, or represents that its use would not infringe privately owned rights. Reference herein to any specific commercial product, process, or service by trade name, trademark, manufacturer, or otherwise, does not necessarily constitute or imply its endorsement, recommendation, or favoring by the United States Government or any agency thereof. The views and opinions of authors expressed herein do not necessarily state or reflect those of the United States Government or any agency thereof.

The work described in this report was performed at Oak Ridge National Laboratory for the U.S. Nuclear Regulatory Commission, under Interagency Agreement 1886-N697-OY with the USDOE, under contract DEAC05-00OR22725 with UT-Battelle. 


\title{
Reliability of Current Biokinetic and Dosimetric Models for Radionuclides: A Pilot Study
}

\author{
Authors: \\ R. W. Leggett \\ K. F. Eckerman \\ R. A. Meck
}

Date Published: August 2008

\author{
Published by \\ OAK RIDGE NATIONAL LABORATORY \\ Oak Ridge, Tennessee 37831 \\ Managed by UT-Battelle, LLC, for the \\ U. S. DEPARTMENT OF ENERGY \\ under contract DE-AC05-00OR227
}




\section{CONTENTS}

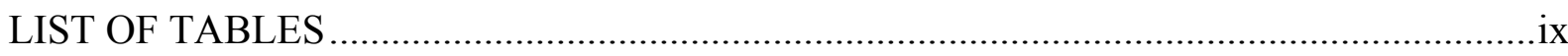

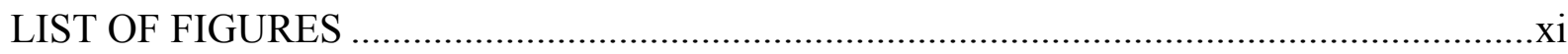

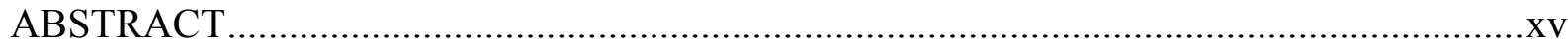

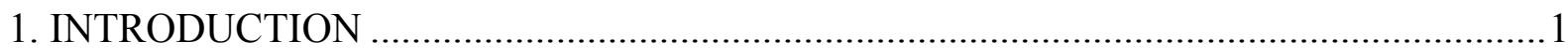

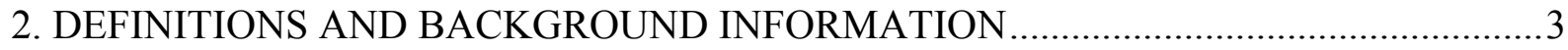

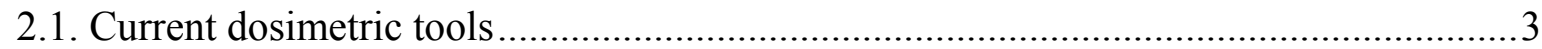

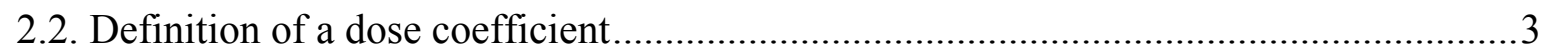

2.3. Definitions of uncertainty and variability .......................................................... 4

2.4. Ambiguity of the term "uncertainty in a dose coefficient" for internal emitters ............5

2.5. Uncertainty in an external dose coefficient.......................................................... 6

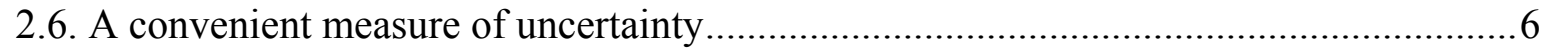

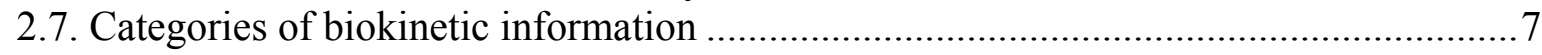

3. RESPIRATORY TRACT MODEL: UNDERLYING DATA AND INITIAL ASSESSMENTS OF VARIABILITY AND UNCERTAINTY …....................... 8

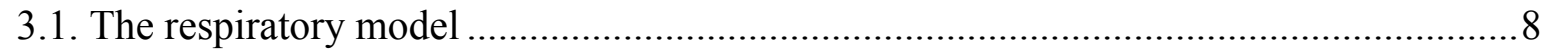

3.2. Basis for generic parameter values of the HRTM .............................................. 11

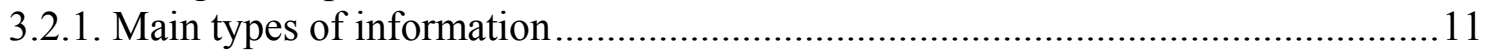

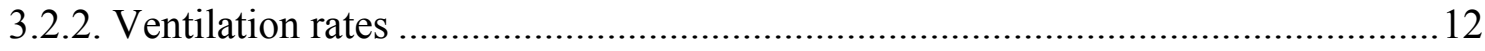

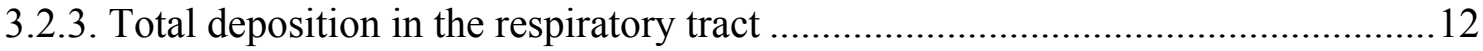

3.2.4. Initial division between the thoracic and extrathoracic regions ......................... 13

3.2.5. Regional deposition in the lungs ............................................................ 14

3.2.6. Clearance of material from the lungs ......................................................... 16

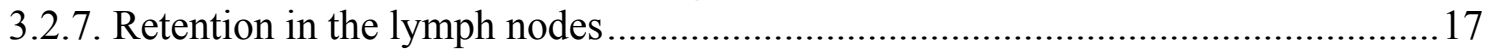

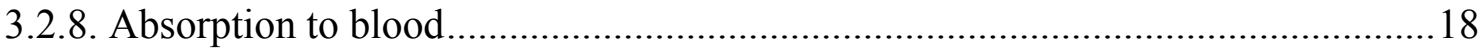

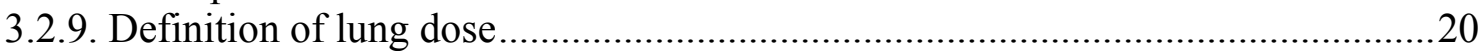

3.3. Existing evaluations of potential errors and uncertainties in HRTM components

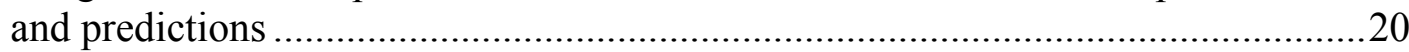

3.3.1. Expert judgments by the developers of the HRTM..........................................20

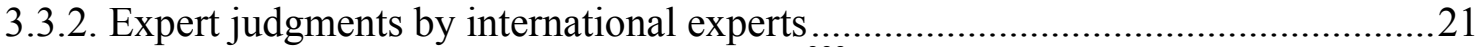

3.3.3. Parameter uncertainty analysis applied to ${ }^{222} \mathrm{Rn}$ daughter dosimetry ..................21

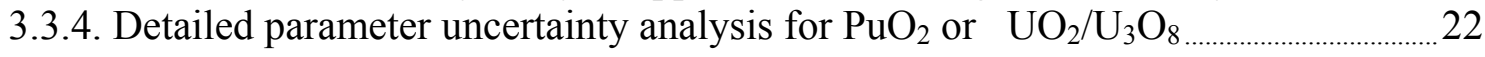

3.3.5. A modification of the analysis of Bolch and coworkers for inhaled $\mathrm{PuO}_{2} \quad \ldots \ldots \ldots .23$

3.3.6. Comparison with an alternate ventilation model...........................................23

3.3.7. Comparison with alternate parameter values for inhaled mercury vapor ............24

3.3.8. Comparison with the NCRP respiratory model.............................................26

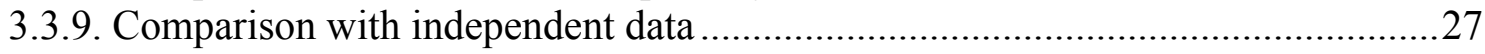




\section{CONTENTS (cont'd)}

4. GASTROINTESTINAL TRACT MODEL: UNDERLYING DATA AND INITIAL ASSESSMENTS OF VARIABILITY AND UNCERTAINTY .................................... 31

4.1. The ICRP's gastrointestinal tract model used to derive current dose coefficients ........31

4.2. The ICRP's updated GI tract model............................................................................32

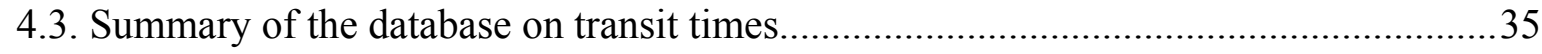

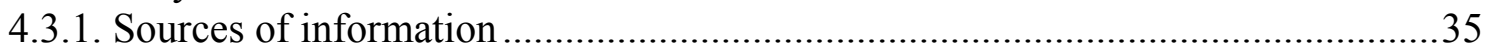

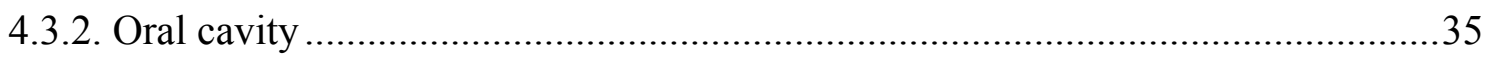

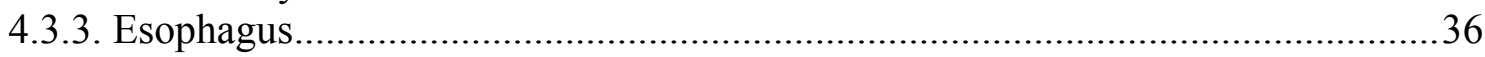

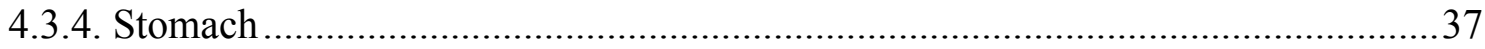

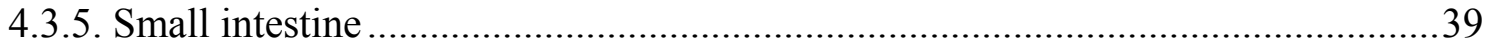

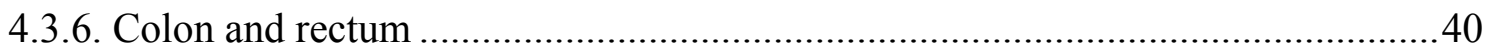

4.4. Contents of segments of the tract and fecal excretion rates .........................................42

4.5. Basis for the dosimetric model of the human alimentary tract ....................................43

4.5.1. Limitations of the GI tract model of ICRP Publication 30 .................................43

4.5.2. A more direct approach taken in the HATM.....................................................43

4.5.3. Illustration of dosimetric data and assumptions: Small intestine ...........................43

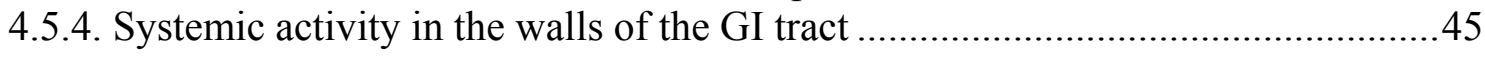

4.5.5. Implications of the HATM for alpha emitters.....................................................46

4.6. Expert judgments of potential errors in HATM features and predictions......................46

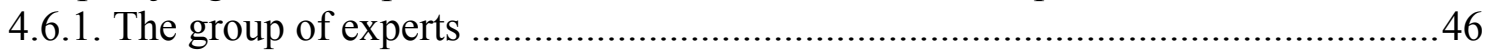

4.6.2. Potential errors associated with limitations in model structure...............................46

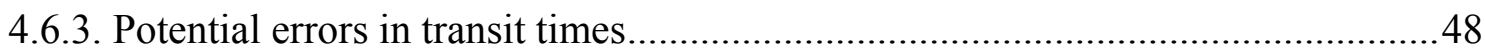

4.6.4. Simplifications of anatomical features of the alimentary tract..............................49

4.6.5. Uncertainties in the location of target regions for cancer induction ......................49

4.6.6. Potential errors in element-specific parameter values of the HATM.....................50

\section{DEMONSTRATION OF DIFFERENT LEVELS OF KNOWLEDGE OF THE} BIOKINETICS AND DOSE PER UNIT INTAKE FOR SPECIFIC

RADIONUCLIDES ………………………………….................................52

5.1. Cesium-137: Level 1 (Highest level of information) .................................................52

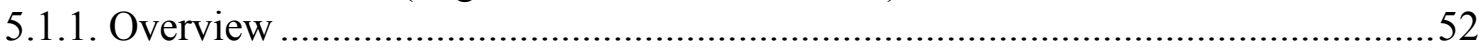

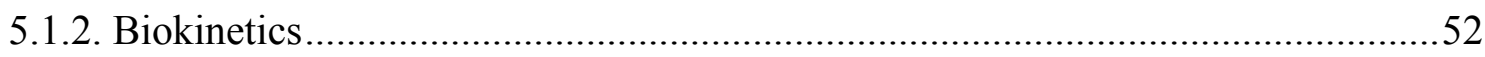

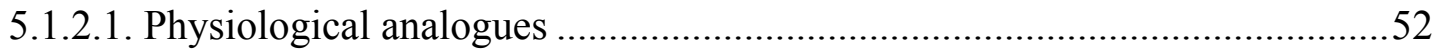

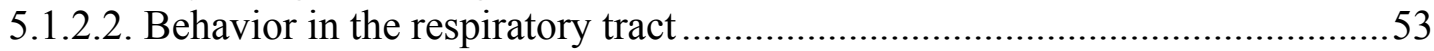

5.1.2.3. Fractional uptake from the gastrointestinal tract..........................................53

5.1.2.4. Systemic biokinetics ..............................................................................54

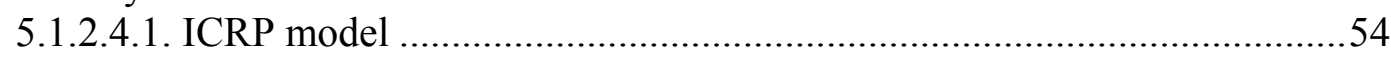

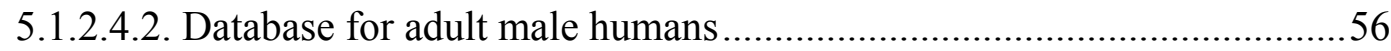

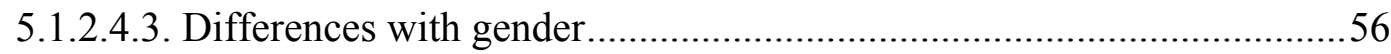

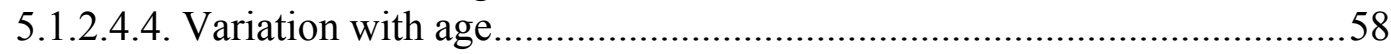

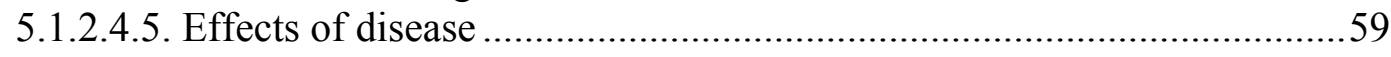

5.1.2.4.6. Behavior of radioactive progeny …………….....................................59 


\section{CONTENTS (cont'd)}

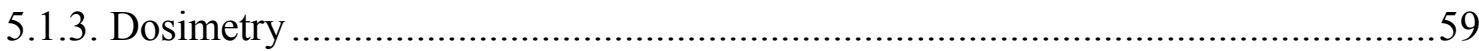

5.1.4. Variability of dose per unit intake or per unit exposure........................................60

5.1.5. Existing uncertainty assessments for intake of ${ }^{137} \mathrm{Cs}$..........................................60

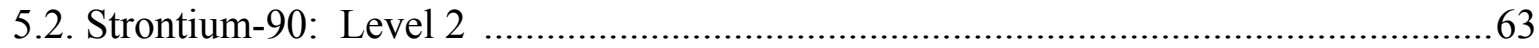

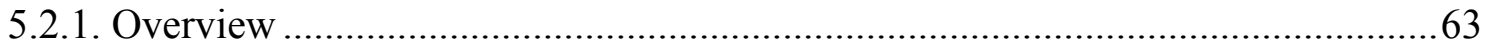

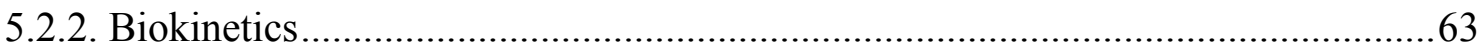

5.2.2.1. Physiological analogues .........................................................................63

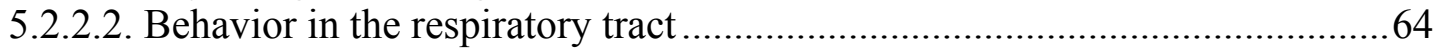

5.2.2.3. Fractional uptake from the gastrointestinal tract...........................................64

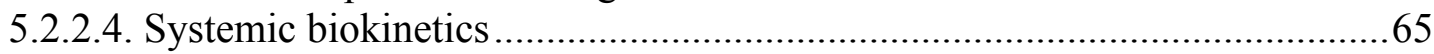

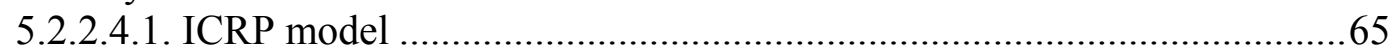

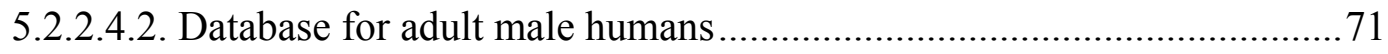

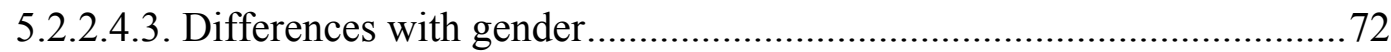

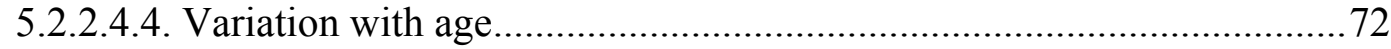

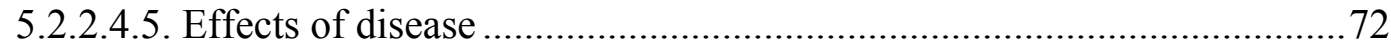

5.2.2.4.6. Biokinetics of radioactive progeny ………..........................................73

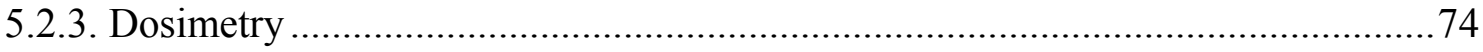

5.2.4. Variability of dose per unit intake or per unit exposure .......................................74

5.2.5. Existing uncertainty assessments for intake of ${ }^{90} \mathrm{Sr}$...........................................74

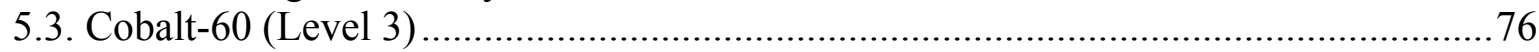

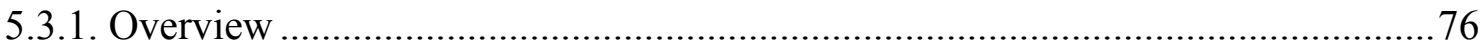

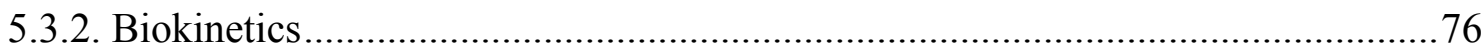

5.3.2.1. Physiological analogues ......................................................................76

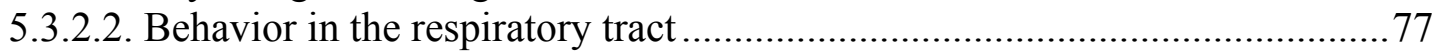

5.3.2.3. Fractional uptake from the gastrointestinal tract ....................................... 78

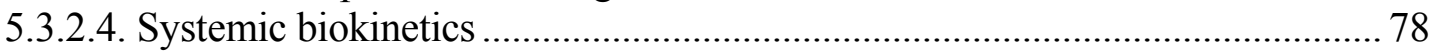

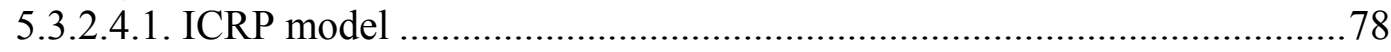

5.3.2.4.2. Summary of biokinetic data on absorbed cobalt....................................79

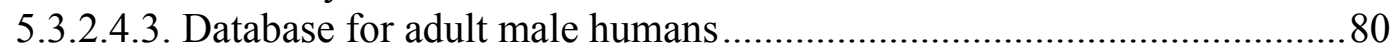

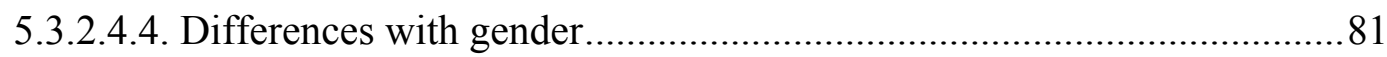

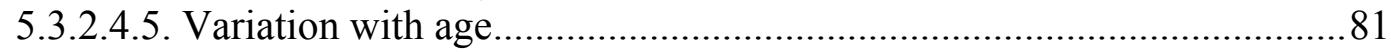

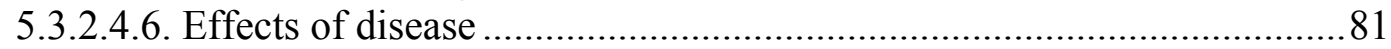

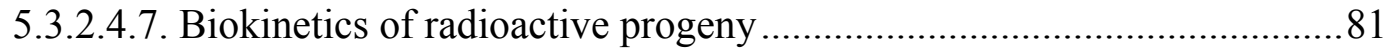

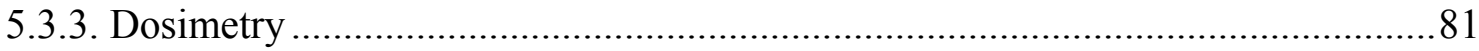

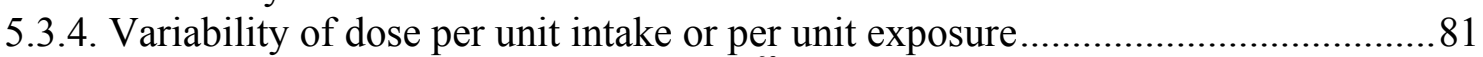

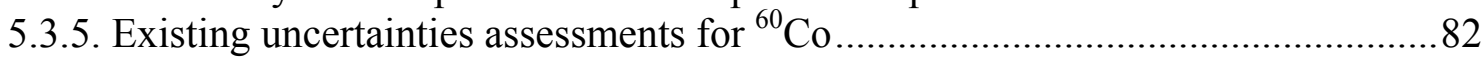

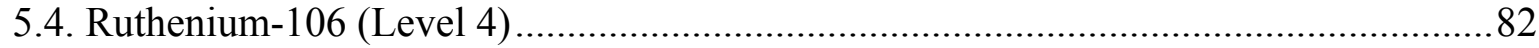

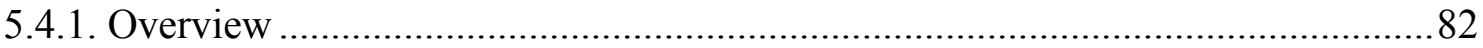

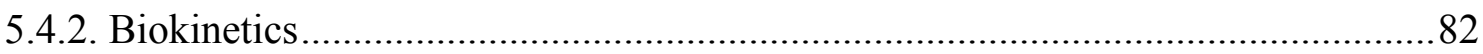

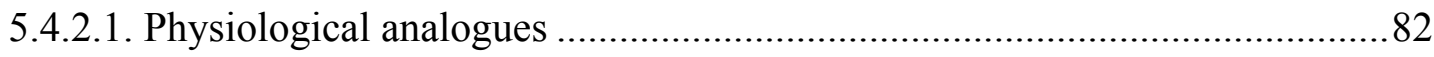

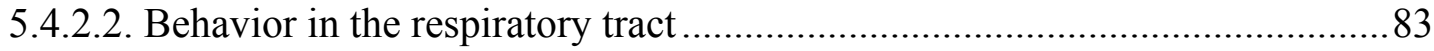

5.4.2.3. Fractional uptake from the gastrointestinal tract............................................83

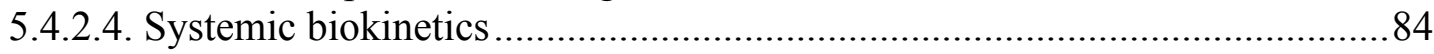




\section{CONTENTS (cont'd)}

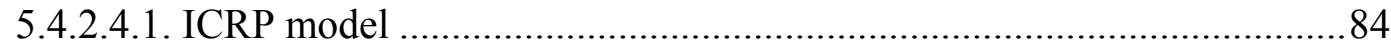

5.4.2.4.2. Summary of biokinetic data on absorbed ruthenium..............................84

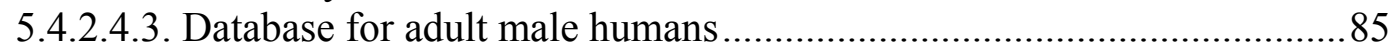

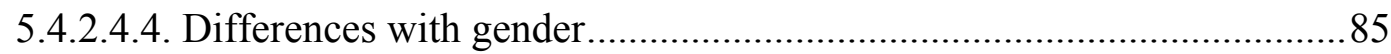

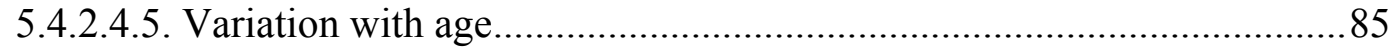

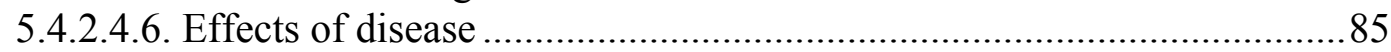

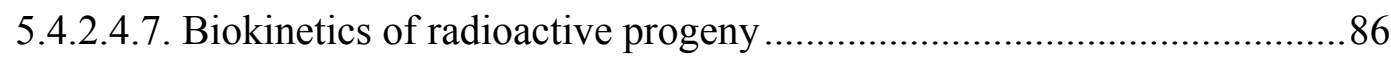

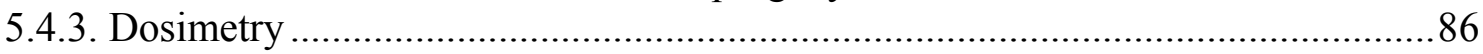

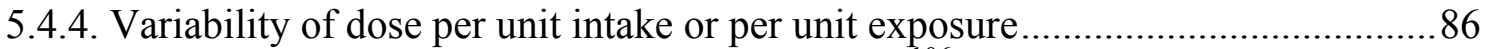

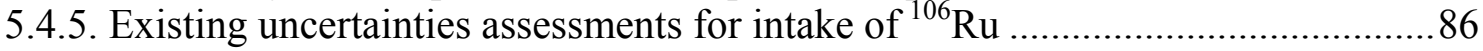

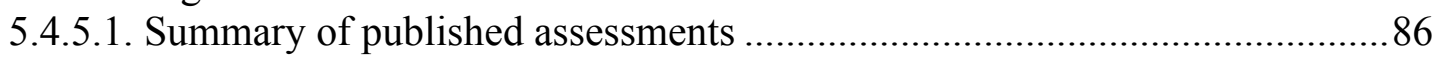

5.4.5.2. A preliminary assessment based on comparison of alternate models ............86

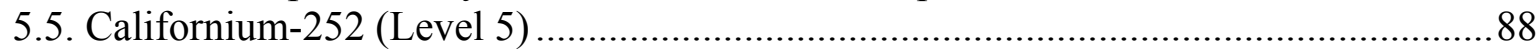

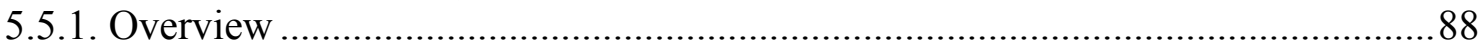

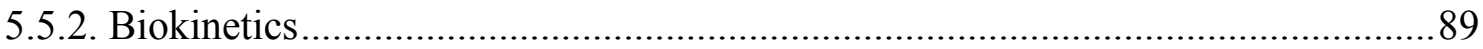

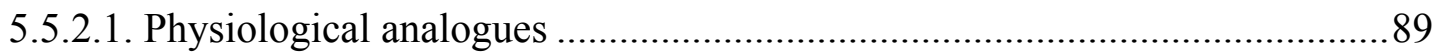

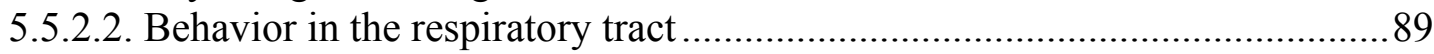

5.5.2.3. Fractional uptake from the gastrointestinal tract...........................................8

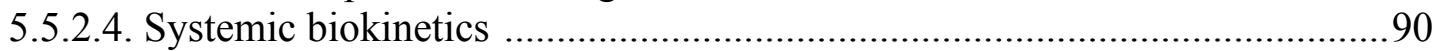

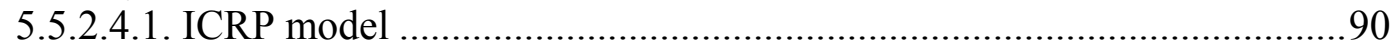

5.5.2.4.2. Summary of biokinetic data on absorbed californium............................99

5.5.2.4.3. Database for adult male humans.......................................................92

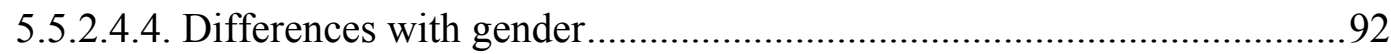

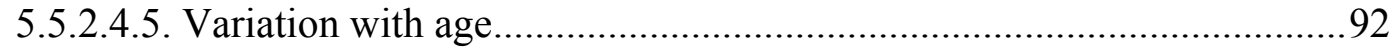

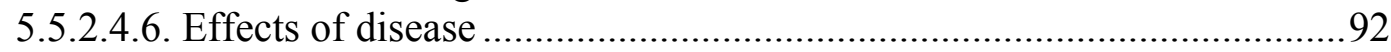

5.4.2.4.7. Biokinetics of radioactive progeny ..................................................92

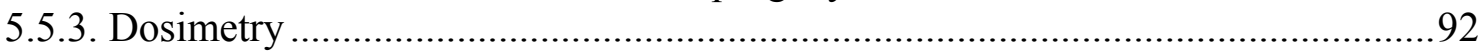

5.5.4. Variability of dose per unit intake or per unit exposure ......................................94

5.5.5. Existing uncertainties assessments for intake of ${ }^{252} \mathrm{Cf}$........................................94

5.5.5.1. Published assessments ..............................................................................94

5.5.5.2. Comparison with an alternate model ............................................................94

\section{DEMONSTRATION OF DIFFERENT LEVELS OF POTENTIAL ERROR IN}

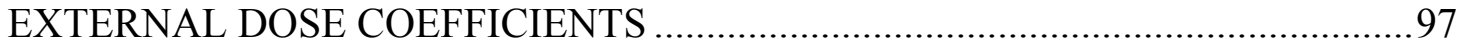

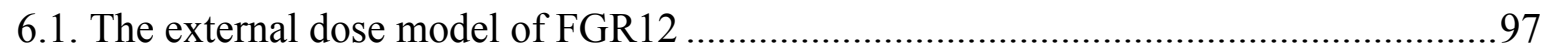

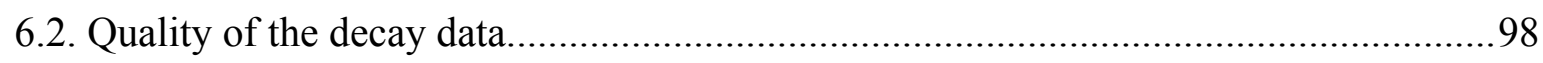

6.3. Potential errors in external dose coefficients for selected radionuclides ......................98

6.4. Variation of external dose with body size................................................................99

\section{DEMONSTRTION OF LEVELS OF POTENTIAL ERROR IN NUCLEAR DECAY} DATA USED IN COMPUTATION OF DOSE COEFFICIENTS …………………....103

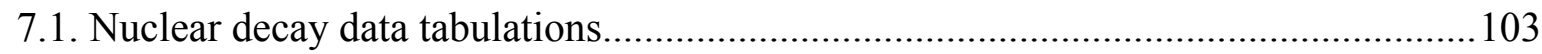

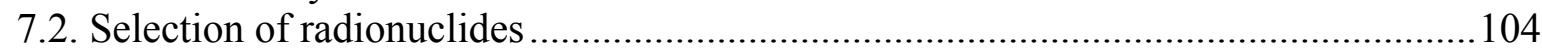

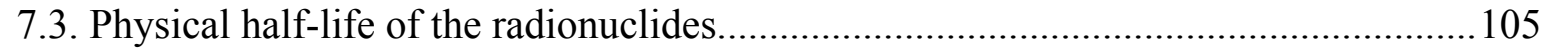




\section{CONTENTS (cont'd)}

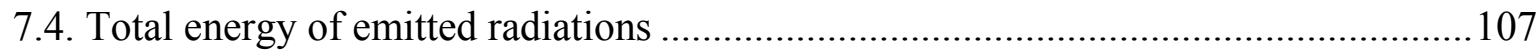

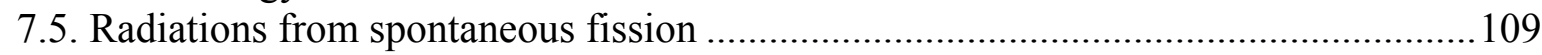

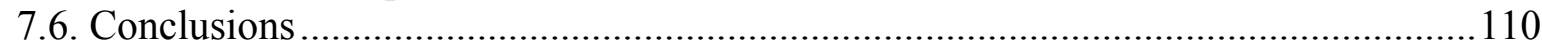

8. RECOMMENDATIONS FOR A COMPREHENSIVE STUDY ….............................111

8.1. Definitions and practical bounds for a comprehensive study ............................... 111

8.1.1. Definitions of uncertainty and variability ................................................. 111

8.1.2. Potential error compared with uncertainty and variability .............................. 111

8.1.3. The need to avoid new dosimetric frontiers ............................................... 112

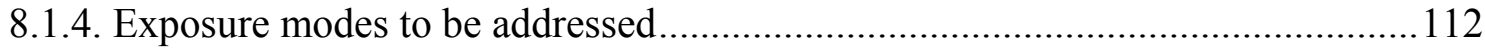

8.1.5. Radionuclides to be addressed ................................................................... 112

8.2. Proposed methods of assessment of potential error, uncertainty, and variability ....... 114

8.2.1. Inhalation or ingestion of radionuclides ................................................ 114

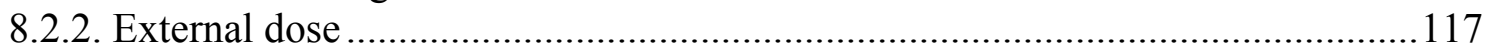

8.3. Estimates of resources required for a comprehensive project................................ 118

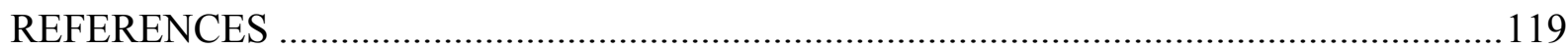




\section{LIST OF TABLES}

Table 3.1. Variation with age in total and regional deposition fractions as a function of age, as predicted by HRTM for inhalation of $1-\mu \mathrm{m}$ particles (AMAD)

Table 3.2. Comparison of dose coefficients for inhalation of mercury vapor by a worker, based on the HRTM with current ICRP parameter values for mercury vapor (ICRP, 1994b) and parameter values proposed by Leggett et al. (2001)....

Table 3.3. Comparison of 50-y committed equivalent dose coefficients for inhalation of ${ }^{234} \mathrm{U}$ based on models for Type $\mathrm{M}$ and Type $\mathrm{S}$ and the $\mathrm{UAl}_{\mathrm{X}}$ model described in this report

Table 3.4. Comparison of effective dose estimates derived with Type M or Type S parameter values with estimates derived from $\mathrm{UAl}_{\mathrm{x}}$ - specific parameter values developed by ORNL

Table 4.1. HATM default transit times for luminal contents of the regions of the gastrointestinal tract

Table 4.2. Reference values for contents of segments of the alimentary tract and daily fecal excretion

Table 4.3. Target cell depths and masses for regions of the HATM for adult males

Table 4.4. Differences in dose coefficients for the colon, compared to the default case, resulting from considerations of target depth in the mucosa, considering ingestion by adult males

Table 5.1. Typical age- and gender-specific long-term retention half-times for cesium in the human body

Table 5.2. Expected differences with age and gender in dose from ingestion of ${ }^{137} \mathrm{Cs}$

Table 5.3. Estimated effective dose from ingestion of $1 \mathrm{~Bq}$ of ${ }^{90} \mathrm{Sr}$ for different groups, relative to the estimated value for a young adult male

Table 5.4. Comparison of injection dose coefficients for ${ }^{106} \mathrm{Ru}$ (adult male) based on three different biokinetic models for ruthenium described in the text

Table 5.5. Species differences in the early distribution of ${ }^{252} \mathrm{Cf}$ injected as citrate

Table 6.1. Comparative external dose coefficients from FGR12 for exposure to contaminated ground surface 


\section{LIST OF TABLES (cont'd)}

Table 7.1. Radionuclides for which updated half-lives are substantially different from

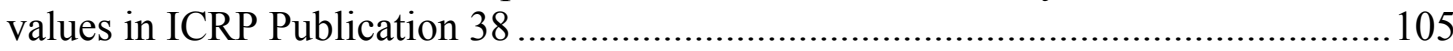

Table 7.2. Comparison of half-lives given in ICRP Publication 38 with more recent measurements by NIST

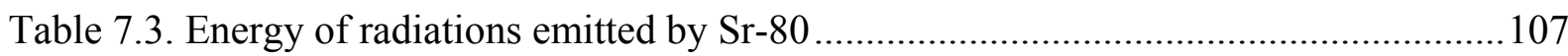

Table 8.1. Primary radionuclides and exposure modes associated with the Chernobyl accident

Table 8.2. Central estimates and plausible ranges of 50-y-committed doses per unit intake of ${ }^{137} \mathrm{Cs}$

Table 8.3. Variability of biokinetics of $\mathrm{Sr}$ in healthy young adult males 


\section{LIST OF FIGURES}

Figure 2.1. Schematic of ICRP methodology for calculation of dose coefficients for inhalation or ingestion of radionuclides

Figure 3.1. Structure of the ICRP's respiratory tract model.

Figure 3.2. Schematic of treatment of time-dependent dissolution of inhaled particles in the HRTM

Figure 3.3. Variation with age and gender in 24-h average air intake, as estimated by Layton (1993) on the basis of the body's energy requirements

Figure 3.4. Comparison of predictions of the ventilation submodel of the HRTM with values derived from an independent model based on energy requirement.

Figure 3.5. Urinary excretion data for a worker exposed to airborne $\mathrm{UAl}_{\mathrm{x}}$, compared with predictions of the HRTM based on Type M or Type S aerosols

Figure 3.6. Comparison of the predictions of the modified HRTM with urinary $U$ data for a worker who was heavily exposed to $\mathrm{UAl}_{\mathrm{x}}$

Figure 4.1. Structure of the gastrointestinal tract model used by the ICRP since the late 1970s

Figure 4.2. Structure of the HATM .32

Figure 4.3. Effects of age and food type on residence times in the mouth 35

Figure 4.4. Differences with age, food type, and body position in transit times through the lumen of the esophagus.

Figure 4.5. Comparison of reported gastric half-emptying times for solids, a variety of test liquids, and non-caloric liquids in healthy adults.....

Figure 4.6. Comparison of gastric half-emptying times of solids in adult male and female subjects in nine studies.

Figure 4.7. Reported small intestinal transit times in subjects without gastrointestinal disorders.

Figure 4.8. Ranges and overall means of reported colonic transit times for normal children, adult males, and adult females 


\section{LIST OF FIGURES (cont'd)}

Figure 4.9. Summary of reported transit times in right colon, left colon, and rectosigmoid in normal human subjects

Figure 4.10. Schematic of the geometric model used to estimate energy deposition in radiosensitive cells in the walls of tubular regions of the alimentary tract, from decay of radionuclides in the lumen

Figure 5.1. Structure of ICRP's current systemic biokinetic model for cesium

Figure 5.2. Structure of a physiologically based biokinetic model for Cs in the human body

Figure 5.3. Whole-body retention of ingested cesium as predicted by the proposed cesium model update and measured in several adult male humans followed for an extended period

Figure 5.4. Mean long-term half-times for total-body retention of cesium in 14 studies involving healthy adult male subjects

Figure 5.5. Comparative total-body half-times of cesium in adult males and adult females in eight studies

Figure 5.6. Measured ${ }^{137} \mathrm{Cs}$ whole-body retention half-times at different ages. 58

Figure 5.7. ICRP's generic model structure for calcium-like elements, introduced in ICRP Publication 67 and applied in that document or subsequent ICRP documents to calcium, strontium, barium, radium, lead, and uranium

Figure 5.8. Retention of strontium in soft tissues as a function of time after intravenous injection of radiostrontium into human subjects

Figure 5.9. Division of activity between bone and soft tissues as a function of time after intravenous injection of radiostrontium into human subjects

Figure 5.10. Differences with age in whole-body retention of strontium

Figure 5.11. Differences with age in the concentration of ${ }^{90} \mathrm{Sr}$ in human vertebrae during a period of elevated fallout

Figure 5.12. Species comparison of the size of the long-term retention component for inorganic cobalt

Figure 5.13. Species comparison of long-term retention of ruthenium in the total body .85 


\section{LIST OF FIGURES (cont'd)}

Figure 5.14. Systemic biokinetic for ruthenium developed by Runkle et al. (1980) based on data for rats exposed to ${ }^{106} \mathrm{RuO}_{4}$ by inhalation or ingestion ....................................87

Figure 5.15. Observed patterns of urinary excretion of ${ }^{252} \mathrm{Cf}$ following acute inhalation.........90

Figure 5.16. Structure of the proposed model for californium ..............................................95

Figure 5.17. Comparison of predictions of urinary californium based on the systemic biokinetic model for californium given in ICRP Publication 68 and Publication 78 (1997) and a proposed model, assuming intravenous injection of californium at time 0

Figure 6.1.Age-specific equivalent dose to the stomach from monoenergetic photons incident on the body

Figure 6.2. The equivalent dose to the liver of an adult male or female for a mononergetic photon source uniformly distributed in the air or on the ground surface.

Figure 6.3. Age-specific values of the effective dose per unit air kerma for monoenergetic photons incident on the front of the body and on the right side of the body

Figure 6.4. Age-specific values of the effective dose per unit air kerma for monoenergetic photons incident on the body in an isotropic geometry

Figure 7.1. Decay scheme for ${ }^{80} \mathrm{Sr}$ given in ICRP Publication 38 compared with JAERI 1347 
xiv 


\begin{abstract}
This report describes the results of a pilot study of the reliability of the biokinetic and dosimetric models currently used by the U.S. Nuclear Regulatory Commission (NRC) as predictors of dose per unit internal or external exposure to radionuclides. The study examines the feasibility of critically evaluating the accuracy of these models for a comprehensive set of radionuclides of concern to the NRC. Each critical evaluation would include: identification of discrepancies between the models and current databases; characterization of uncertainties in model predictions of dose per unit intake or unit external exposure; characterization of variability in dose per unit intake or unit external exposure; and evaluation of prospects for development of more accurate models. Uncertainty refers here to the level of knowledge of a central value for a population, and variability refers to quantitative differences between different members of a population. This pilot study provides a critical assessment of models for selected radionuclides representing different levels of knowledge of dose per unit exposure. The main conclusions of this study are as follows:
\end{abstract}

(1) To optimize the use of available NRC resources, the full study should focus on radionuclides most frequently encountered in the workplace or environment. A list of 50 radionuclides is proposed.

(2) The reliability of a dose coefficient for inhalation or ingestion of a radionuclide (i.e., an estimate of dose per unit intake) may depend strongly on the specific application. Multiple characterizations of the uncertainty in a dose coefficient for inhalation or ingestion of a radionuclide may be needed for different forms of the radionuclide and different levels of information of that form available to the dose analyst.

(3) A meaningful characterization of variability in dose per unit intake of a radionuclide requires detailed information on the biokinetics of the radionuclide and hence is not feasible for many infrequently studied radionuclides.

(4) The biokinetics of a radionuclide in the human body typically represents the greatest source of uncertainty or variability in dose per unit intake.

(5) Characterization of uncertainty in dose per unit exposure is generally a more straightforward problem for external exposure than for intake of a radionuclide.

(6) For many radionuclides the most important outcome of a large-scale critical evaluation of databases and biokinetic models for radionuclides is expected to be the improvement of current models. Many of the current models do not fully or accurately reflect available radiobiological or physiological information, either because the models are outdated or because they were based on selective or uncritical use of data or inadequate model structures. In such cases the models should be replaced with physiologically realistic models that incorporate a wider spectrum of information. 



\subsection{INTRODUCTION}

This report describes the results of a pilot study of the reliability of the biokinetic and dosimetric models currently used by the U.S. Nuclear Regulatory Commission (NRC) as predictors of dose per unit internal or external exposure to radionuclides. The study examines the feasibility of critically evaluating the accuracy of these models for a comprehensive set of radionuclides of concern to the NRC. Each critical evaluation would include: identification of discrepancies between the models and current databases; characterization of uncertainties in model predictions of dose per unit intake or unit external exposure; characterization of variability in dose per unit intake or unit external exposure; and evaluation of prospects for development of more accurate models. Uncertainty refers here to the level of knowledge of a central value for a population, and variability refers to quantitative differences between different members of a population.

This pilot study provides a critical assessment of biokinetic and dosimetric models for selected radionuclides representing different levels of knowledge of dose per unit exposure. Estimates are made of the resources needed to implement a large-scale analysis.

The main body of the report is divided as follows. Section 2 gives definitions and background information used in subsequent discussions of different data types, uncertainties, variability, and different approaches to uncertainty analysis. Sections 3 and 4 provide extensive illustrations of the type and quality of data underlying the two main "generic" models used by the International Commission on Radiological Protection (ICRP) in dose calculations for internal emitters: the respiratory tract model (Section 3), and the gastrointestinal tract model (Section 4). The respiratory and gastrointestinal models are referred to as generic models because the bases, features, and predictions of these models are in many respects independent of the radionuclide. The basis for generic features of each of these two models is discussed at length due to the importance of these two regions as interfaces between the environment and interior of the human body, and because estimated doses to tissues in these two regions based on current models often dominate the effective dose or projected risk from intake of radionuclides. Section 5 demonstrates different levels of information of the biokinetics in humans and dose per unit intake for specific radionuclides, summarizes information on the variability of biokinetics and dose per unit intake or per unit exposure in each case, and critically evaluates uncertainty assessments on those cases previously addressed in the literature. Five main radionuclides are considered in Section 5. These are listed below in order of decreasing direct knowledge of their behavior in humans.

${ }^{137} \mathrm{Cs}$ : Level 1 - The database contains extensive, high quality data on the behavior of cesium in human subjects, supported by numerous observations on the behavior of cesium in laboratory animals. There is also extensive information on the biokinetics of physiological analogues of cesium in humans and laboratory animals. A revised model described in Section 5 based on physiological considerations provides a good fit to the data. 
${ }^{90} \mathrm{Sr}$ : Level 2 - Extensive data are available for human subjects and laboratory animals, but data for strontium are more variable and less easily interpreted overall than data for cesium. For ${ }^{90} \mathrm{Sr}$ there is increased reliance on indirect information, particularly data for physiological analogues. Conversion from activity to dose involves only small errors for most tissues but somewhat larger errors for the most highly irradiated tissues, bone surfaces and red marrow.

${ }^{60}$ Co: Level 3 - Human data include external measurements of retention and also excretion data from a few controlled studies. The internal distribution of cobalt has been studied in laboratory animals but extrapolation to humans is complicated by apparent differences between species and the chemical form administered. Cobalt has no close physiological analogues. Conversion from activity to dose is straightforward for ${ }^{60} \mathrm{Co}$.

${ }^{106} \mathrm{Ru}$ : Level 4 - Modeling of ruthenium biokinetics is based on data for laboratory animals, supplemented with limited information for man. Suspected physiological analogues of ruthenium also have sparse databases. Conversion from activity to dose appears to introduce relatively small errors.

${ }^{252} \mathrm{Cf}$ : Level 5 - The biokinetics of californium must be modeled on the basis of animal data, including information on the comparative behavior of californium and other transuranic elements. There are also important potential errors associated with the dosimetric model used to convert from ${ }^{252} \mathrm{Cf}$ activity to equivalent dose to tissues. These potential errors arise in part from a simplistic and somewhat uncertain representation of dose due to spontaneous fission and in part from uncertainties regarding the radiobiological effectiveness (RBE) of alpha particles and neutrons arising from decay of ${ }^{252} \mathrm{Cf}$.

Section 6 discusses the basis for external dose coefficients and potential errors associated with applications of those coefficients for selected radionuclides. The nuclear decay data used in the development of dosimetric models are discussed briefly in Sections 3-6 and are addressed in more detail in Section 7. Although nuclear decay data generally represent only a minor source of error in dose estimates, examples are given in Section 7 to illustrate that these data can be an important source of error in dose estimates for some infrequently studied radionuclides. Section 8 proposes a general approach for assessing and in many cases reducing potential errors in dose estimates for a selected set of important radionuclides. A list of 50 radionuclides is proposed for consideration, based on their importance as indicated by past experience or the potential for elevated exposures in the future. Estimates of the time, funds, and other resources required to implement a larger project are provided. 


\section{DEFINITIONS AND BACKGROUND INFORMATION}

This section defines the "current dosimetric tools" addressed in this report, the term "dose coefficient" for intake of a radionuclide or external exposure to a radionuclide in a given medium, and the terms " uncertainty and variability" as used in this report. Examples are given to show that the commonly used term, "uncertainty in a dose coefficient", is ambiguous for the case of ingestion or inhalation of a radionuclide, without specific information or assumptions concerning the form of the radionuclide taken into the body. A measure of uncertainty in biokinetic or dosimetric quantities is described. Different categories of biokinetic data are defined and assigned abbreviated names for use in later sections.

\subsection{Current dosimetric tools}

Unless otherwise indicated, the current dosimetric tools referred to in this report are the tools for internal dosimetry currently recommended by the ICRP, including biokinetic and dosimetric models as well as dose coefficients, and the external dose coefficients given in Federal Guidance Report No. 12 (FGR12) (EPA, 1993).

\subsection{Definition of a dose coefficient}

To place all ionizing radiations on a common scale with regard to their potential health detriment, the ICRP uses quantities called the equivalent dose and the effective dose. The equivalent dose is the absorbed dose averaged over an organ or tissue and multiplied by a radiation weighting factor, $\mathrm{W}_{\mathrm{R}}$, that reflects the relative biological effectiveness of the type and energy of radiation causing the dose. The effective dose takes into account that the relationship between equivalent dose and the probability of radiogenic effects depends on the organ or tissue irradiated. The effective dose is a weighted sum of equivalent doses to radiosensitive tissues, with the tissue weighting factors, $\mathrm{w}_{\mathrm{T}}$, representing the relative contribution of the different tissues to the total detriment for the case of uniform irradiation of the whole body.

For an internally deposited radionuclide, the time integral of the equivalent-dose rate, beginning at intake, is called the committed equivalent dose. If not otherwise specified, the integration period is $50 \mathrm{y}$ for intake by adults and to age $70 \mathrm{y}$ for intake by children. The committed effective dose is the weighted sum of the committed equivalent doses to radiosensitive tissues. A committed tissue equivalent dose per unit intake or a committed effective dose per unit intake for a specified radionuclide, exposure mode, and population (e.g., a certain age group) is referred to as a dose coefficient.

The steps involved in the calculation of dose coefficients for inhalation or ingestion of radionuclides are indicated in the flow chart in Figure 2.1. The estimation of absorbed dose to radiosensitive tissues involves application of models, commonly referred to as biokinetic models, that describe the biological behavior of activity entering the body and a second set of models, referred to in this report as "dosimetric models", that describe 
radiation transport from the point of decay of radionuclides and resulting deposition of ionizing energy in tissues. Once the absorbed doses have been calculated, the effective dose is computed as an arithmetic combination of the absorbed doses, radiationweighting factors $\left(\mathrm{w}_{\mathrm{R}}\right)$ that represent the radiobiological effectiveness $(\mathrm{RBE})$ of alpha particles, fission fragments, or neutrons relative to gamma or beta radiation, and tissueweighting factors $\left(\mathrm{w}_{\mathrm{T}}\right)$ that represent relative detriment to different tissues from uniform whole-body irradiation.

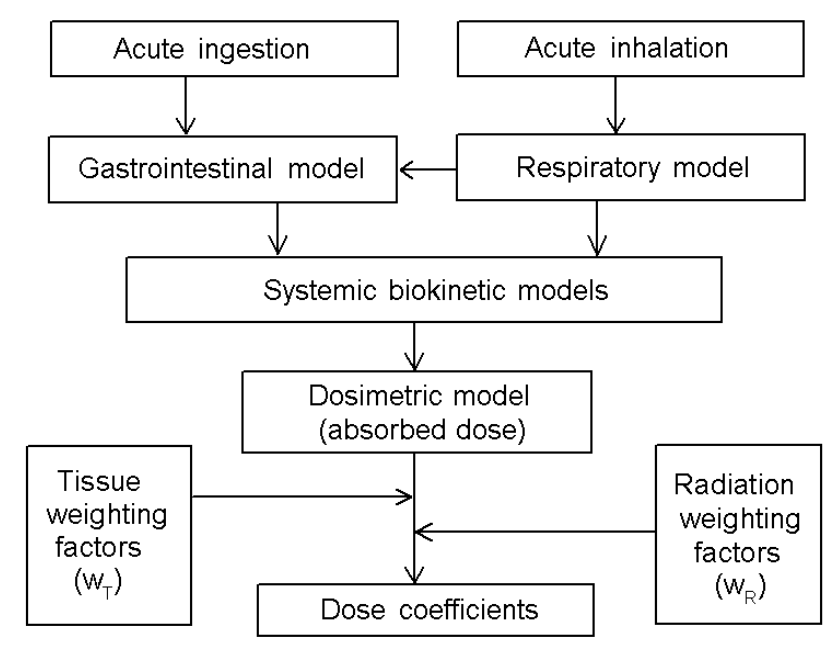

Figure 2.1. Schematic of ICRP methodology for calculation of dose coefficients for inhalation or ingestion of radionuclides

An external dose coefficient is a reference value for dose rate to a tissue or effective dose rate per unit concentration of the radionuclide in an environmental medium. The external dose coefficients given in FGR12 (EPA, 1993) are based on idealized exposure geometries and a mathematical phantom representing a reference adult male standing in an upright position at the air-ground interface. In FGR 12, dose coefficients are provided for radionuclides in different environmental media including: air submersion; exposure to contaminated ground surface; and exposure to soil contaminated to a depth of $1 \mathrm{~cm}, 5$ $\mathrm{cm}, 15 \mathrm{~cm}$, or infinite depth.

\subsection{Definitions of uncertainty and variability}

The biokinetic and dosimetric models used in ICRP publications and the dose coefficients derived from those models are intended to represent typical or "reference" members of the population. The biological behavior of some radionuclides is reasonably well established but in other cases must be predicted on the basis of sparse or inconsistent data. Additionally, some components of the ICRP's dosimetric models involve considerably simplified anatomical structures and assumptions concerning energy deposition, leading to an uncertain characterization of the absorbed dose to the tissues at 
risk. Thus, there is some uncertainty as to whether the components of the biokinetic and dosimetric models and the dose coefficients derived from those models accurately represent typical members of the population, and the level of uncertainty varies from one radionuclide to another.

In this report, "uncertainty" refers to the level of confidence that can be placed in a given biokinetic or dosimetric quantity as an estimate of the central value. Usually, the central value is taken to be an arithmetic or geometric mean for the population.

The uncertainty in the central value of a quantity in the population should not be confused with the "variability" of that feature in the population. Variability refers to quantitative differences between different members of a population under similar conditions (inter-individual variability) or within an individual under different conditions (intra-individual variability). For example, the transit time of material through the colon may differ between two persons of the same size, race, age, and gender and having identical diets (inter-individual variability) or may differ in the same person at different times due to changes in diet, state of health, or other conditions (intra-individual variability).

\subsection{Ambiguity of the term "uncertainty in a dose coefficient" for internal emitters}

It is common to refer to "the uncertainty in a dose coefficient", and a number of investigators have addressed this topic for specific radionuclides and intake modes. The published papers generally do not clearly define what is meant by "uncertainty". In many cases, it appears from the analytical methods that the reference may be to an unknown individual.

Whether the reference is to a central estimate or an individual, the problem arises that "the uncertainty in a dose coefficient" is not a well defined concept for internal emitters. The difficulty is that the level of confidence that can be placed in a dose coefficient may vary considerably from one application to another, depending on the form of the radionuclide and the level of knowledge concerning that form. Suppose, for example, that an inhalation dose coefficient for ${ }^{90} \mathrm{Sr}$, Type $\mathrm{F}$, is used to estimate tissue doses to a worker exposed for a known period to a known concentration of ${ }^{90} \mathrm{Sr}$ in air. Uncertainties in the derived doses would be relatively small if the physical and chemical form of airborne ${ }^{90} \mathrm{Sr}$ are known and indicated by the literature to be consistent with the ICRP's model for Type F. The uncertainties would be larger if available information is weak but suggestive of Type $\mathrm{F}$ material and still larger if Type $\mathrm{F}$ is applied as a typical absorption type for ${ }^{90} \mathrm{Sr}$ in the absence of any specific information on the form of inhaled material.

One could define the uncertainty in a dose coefficient in terms of a confidence level for arbitrary use of that value, but that would substantially overstate the uncertainties associated with dose estimates in many cases. For example, a later section discusses the relatively high level of confidence that can be placed in ingestion dose coefficients for ${ }^{137} \mathrm{Cs}$ in most situations but not all situations. Most forms of ${ }^{137} \mathrm{Cs}$ are readily absorbed from the gastrointestinal tract to blood, and high confidence can be placed in the dose 
coefficients when applied to these forms. It seems misleading to assign a large uncertainty to the dose coefficient for ingested ${ }^{137} \mathrm{Cs}$ simply because that coefficient may occasionally be applied to a form of ${ }^{137} \mathrm{Cs}$ for which the level of absorption to blood is highly uncertain.

A more workable solution would be to provide uncertainty statements for a few different levels of knowledge of the form of the radionuclide. For example, an uncertainty statement could be provided for an ingestion dose coefficient for ${ }^{137} \mathrm{Cs}$ under the assumption that the radionuclide is incorporated in food or ingested in soluble inorganic form, and an alternate uncertainty statement could be derived for arbitrary use of the dose coefficient.

\subsection{Uncertainty in an external dose coefficient}

The "uncertainty in a dose coefficient" is easier to define for an external dose coefficient than for an inhalation or ingestion dose coefficient, because the physical or chemical form of the radionuclide generally is not relevant to the case of external exposure. The problem of estimating external dose rates from contaminated air, soil, or ground surfaces was divided into two steps: (1) the calculation of the radiation field incident on the surface of the body and (2) calculation of organ dose rates due to a body surface source. The total uncertainty in an external dose coefficient for a specific radionuclide, exposure scenario (e.g., standing on a contaminated ground surface), and tissue would reflect the uncertainty in the radiation field incident upon the body and uncertainties in dose rates to specific tissues due to a body surface source.

\subsection{A convenient measure of uncertainty}

It is often convenient to use a shorthand description to summarize conclusions concerning the current level of knowledge of specific biokinetic and dosimetric quantities. The approach used here is consistent with a method used in Federal Guidance Report No. 13 (FGR13) (EPA, 1999). That is, judgments concerning uncertainties in quantities are sometimes summarized in terms of a subjective confidence interval [A,B] or an associated uncertainty factor (UF). The uncertainty in a given quantity is expressed in terms of a subjective confidence interval, that is, an interval of positive values, [A,B], such that the true but unknown value is judged with reasonable confidence to lie between A and B. Here, "reasonable confidence" is defined as a subjective confidence level of $90 \%$. That is, it is judged that there is only a small probability (about $5 \%$ ) that the true value is less than A and only a small probability (about 5\%) that it is greater than B. For purposes of comparing levels of uncertainty of model components that are expressed in different terms or have different orders of magnitude, it is sometimes convenient to apply the concept of an uncertainty factor (UF). An uncertainty factor for a quantity with subjective confidence interval $[\mathrm{A}, \mathrm{B}]$ is defined as $(\mathrm{B} / \mathrm{A})^{1 / 2}$. The quantity is considered to be known within a factor of $(B / A)^{1 / 2}$ in the sense that all values in the interval are within a factor of $(\mathrm{B} / \mathrm{A})^{1 / 2}$ of the geometric mean of $\mathrm{A}$ and $\mathrm{B}$. 
The description of uncertainties in terms of uncertainty factors is a convenient way of summarizing conclusions regarding uncertainties in model components. It has no implications with regard to the central value or the distribution of possible values of a model component.

\subsection{Categories of biokinetic information}

Throughout, the following abbreviations are used for different types of biokinetic data used in the construction of biokinetic models for an element or compound. The "substance of interest" referred to in these abbreviations is usually but not always a specific element or compound. For example, for studies of fractional deposition of inhaled particulates in the respiratory tract, the "substance of interest" is essentially any particulate of specified dimensions, such as AMAD.

- H1: direct information on humans, i.e., quantitative measurements of the substance of interest in human subjects.

- H2: observations of the behavior of chemically similar or physiologically related substances in human subjects.

- A1: observations of the behavior of the substance of interest in non-human species.

- A2: observations of the behavior of chemically similar or physiologically related substances in non-human species.

- P: Physiological or anatomical information, e.g., the turnover rate of water in the body as one estimate of the retention time of tritium taken into the body as tritiated water, or the turnover rate of cortical or trabecular bone as an estimate of the retention time of plutonium in bone of that type.

- in vitro data

Although $\mathrm{H} 1$ is the preferred type of information for construction of biokinetic models for man, direct observations of an element in human subjects often has one or more of the following limitations: small study groups, coupled with potentially large inter-subject variability in the biokinetics of an element; short observation periods, coupled with potentially large intra-subject variability; use of unhealthy subjects whose diseases may alter the biokinetics of the element; paucity of observations for women and children; collection of small, potentially non-representative samples of tissue; inaccuracies in measurement techniques; uncertainty in the pattern or level of intake of the element; atypical study conditions; and inconsistency in reported values. For such reasons, development of biokinetic models for many elements relies heavily and sometimes completely on indirect sources of information (H2, A1, A2, P, and in vitro). Information on chemical families as a whole (H2 or A2) is particularly important for purposes of building a model structure, because we rely heavily on collective information from a set of physiological analogues as well as comparisons with essential elements. 


\section{RESPIRATORY TRACT MODEL: UNDERLYING DATA AND INITIAL ASSESSMENTS OF VARIABILITY AND UNCERTAINTY}

This section describes the structure of the ICRP's Human Respiratory Tract Model (HRTM) and the basis for default parameter values of the model. Several examples of potential errors or uncertainties in HRTM predictions are given. The examples address:

0 uncertainties in components of the HRTM as assessed by its developers

0 uncertainties in predictions of the HRTM as assessed in an expert elicitation study

0 uncertainties in predictions of the HRTM for three cases ( inhaled ${ }^{222} \mathrm{Rn}$ daughters, $\mathrm{PuO}_{2}$, or $\mathrm{UO}_{2} / \mathrm{U}_{3} \mathrm{O}_{8}$ ) as assessed by standard parameter uncertainty analysis

o uncertainties in HRTM default values for 24-hour average ventilation rate as revealed by comparison with an alternate model

0 uncertainties in predictions of the HRTM as indicated by comparison with predictions of an alternate respiratory model developed by a committee of the National Council on Radiation Protection and Measurements (NCRP)

o reduction in potential errors of the HRTM for specific materials through critical evaluations of the database as illustrated by a recently published review of information on inhaled mercury vapor

o reduction in potential errors of the HRTM for specific materials by use of sitespecific information as illustrated by a recently published case study of inhalation of $\mathrm{UAl}_{\mathrm{x}}$ by workers

\subsection{The respiratory model}

Current understanding of the behavior of inhaled radionuclides in the human respiratory tract and of resulting radiation doses to the tissues of the tract is discussed here within the framework of the ICRP's HRTM. The information used to develop the HTRM is described at length in ICRP Publication 66 (1994a). Guidance on application of the HRTM is given in Publication 66 and in more recent open literature publications and an ICRP guidance document (ICRP, 2002a).

The database underlying the HRTM is large, and a prohibitive amount of time and effort would be required to provide a reasonably complete bibliography in a letter report. The reader is referred to the bibliographies given in ICRP documents (1994a, 2002a) and in papers by Bailey and coworkers $(1994,2003)$. Original references are listed in this section only when not cited in those reports and papers or when very specific information from a given study is used here.

The structure of the HRTM is shown in Figure 3.1. The HRTM Task Group that developed this model incorporated as many anatomically and physiologically realistic features as feasible. Nevertheless, the model includes some empirically based components. For example, the alveolar region is arbitrarily divided into three compartments to describe different phases of clearance from this region. Thus, 
compartments within the AI region are defined on a kinetic basis rather than an anatomical or physiological basis.

The HRTM divides the respiratory system into extrathoracic (ET) and thoracic tissues. The airways of the ET region are further divided into the anterior nasal passages in which deposits are removed by extrinsic means such as nose blowing and the posterior nasal passages (nasopharynx, oropharynx, and the larynx) from which deposits are swallowed or absorbed to blood. The airways of the thorax include the bronchi (BB), bronchioles (bb), and alveolar interstitium (AI). Material deposited in the thoracic airways is cleared into blood by absorption, to the gastrointestinal tract by mechanical processes (i.e., transported upward and swallowed), and to the regional lymph nodes via lymphatic channels. In Figure 3.1, reference values for particle transport rate constants are shown beside the arrows and are in units of $\mathrm{d}^{-1}$.

For an inhaled compound, the mechanical clearances of particles indicated in Figure 3.1 are in competition with dissolution rates and absorption to blood, which depend on the element and the chemical and physical form in which it is inhaled. Material-specific dissolution rates have been developed for some radionuclides, usually on the basis of in vitro dissolution studies or interpretation of measured clearance times from the lungs in laboratory animals (ICRP, 2002a).

In most applications of the HRTM, a radionuclide inhaled as a particulate is assigned to one of three default absorption types: Type F (fast dissolution and a high level of absorption to blood), Type $\mathrm{M}$ (an intermediate rate of dissolution and an intermediate level of absorption to blood), and Type $\mathrm{S}$ (slow dissolution and a low level of absorption to blood). The user selects Type F, M, or S based on ICRP recommendations or independent interpretation of available element-specific information. Sufficient information often is available in the literature to associate one of the three default absorption types to a given form of a radionuclide, but information usually is not sufficient to develop material-specific parameter values.

A time-dependent dissolution rate in the respiratory tract is modeled by using one set of compartments representing an initial dissolution rate and a parallel set (not shown in Figure 3.1) representing a final dissolution rate. As indicated in Figure 3.2, material deposited in a compartment representing the initial state dissolves and is absorbed to blood at a constant rate $s_{\mathrm{p}}$ but is simultaneously transferred at a rate $\mathrm{s}_{\mathrm{pt}}$ to a corresponding compartment representing a transformed state. Particles in the transformed state have a dissolution rate $s_{t}$. With this system, the initial dissolution rate is approximately $s_{\mathrm{p}}$ and the final dissolution rate is approximately $\mathrm{s}_{\mathrm{t}}$.

It is assumed that transformed particles are cleared from a compartment at the same rate as the particles in initial state. More generally, all of the particle transport rates indicated in Figure 3.1 (e.g., $0.0001 \mathrm{~d}^{-1}$ from $\mathrm{AI}_{3}$ to $\mathrm{bb}_{1}$ ) are assumed to be independent of the material, although the structure of the model enables particle transport rates to be modified when information is available. As discussed later, the assumption of material- 
independent mechanical transport rates has a physiological basis or empirical support for some but not all regions of the respiratory tract.

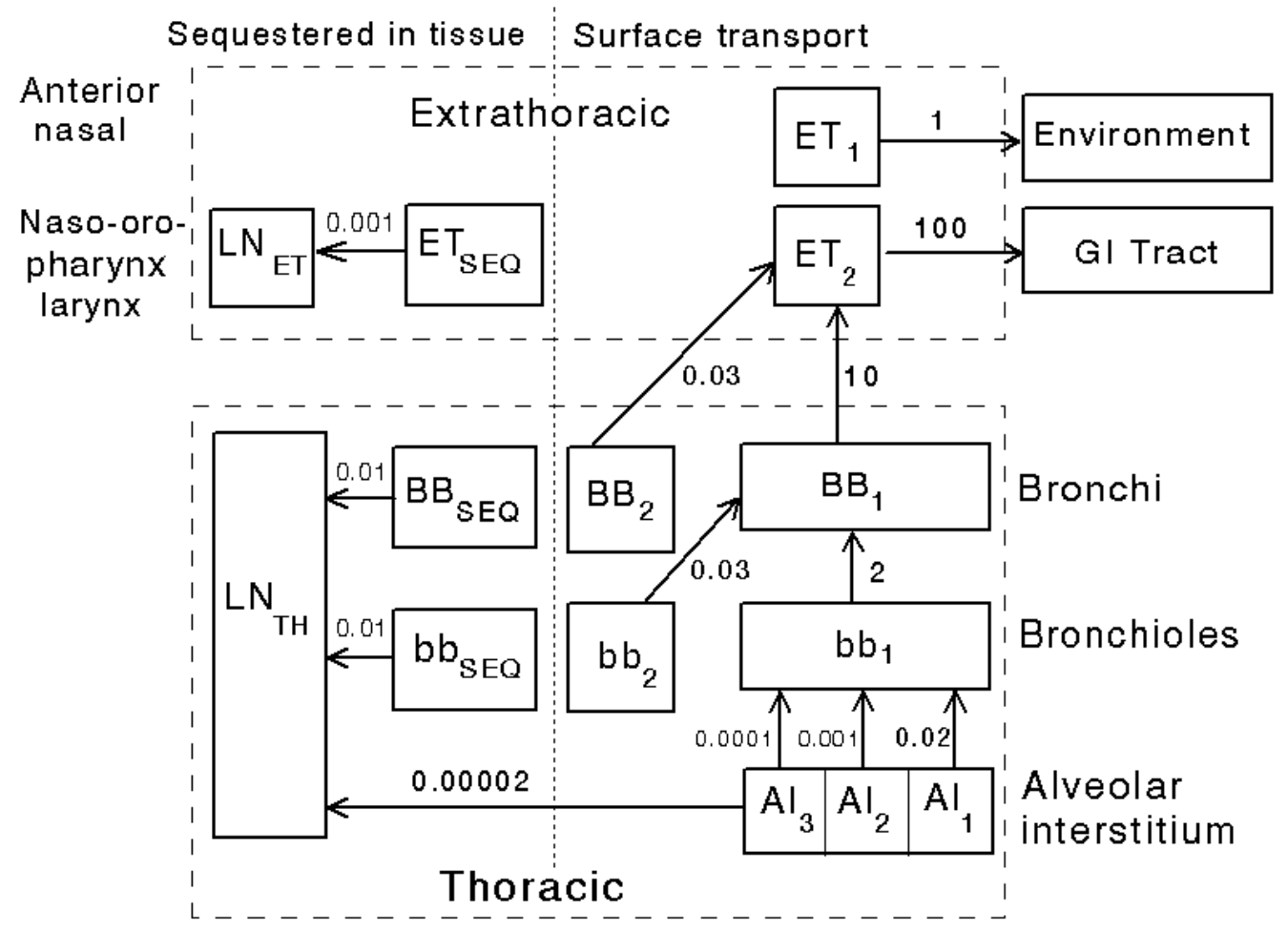

Figure 3.1. Structure of the ICRP's respiratory tract model (ICRP, 1994a) (numbers beside or above the arrows indicate particle transport rates $\left(d^{-1}\right) ; A I=$ alveolar interstitium, $B B=$ bronchi, $b b=$ bronchioles, $\mathrm{ET}=$ extrathoracic, $\mathrm{LN}=$ lymph nodes, $\mathrm{SEQ}=$ sequestered, $\mathrm{TH}$ $=$ thoracic)

The system indicated in Figure 3.2 can be used to depict an increasing rate of dissolution but generally has been used to describe a decreasing dissolution rate with a rapid phase of dissolution immediately after intake. The latter pattern is predicted by the ICRP's default parameter values for fast $(\mathrm{F})$, moderate $(\mathrm{M})$, and slow $(\mathrm{S})$ dissolution.

Deposition of inhaled material in the respiratory tract is defined as the portion of inhaled material remaining after exhalation, including any that is instantaneously absorbed to blood. For radionuclides inhaled as solid or liquid particles, regional deposition is assumed to be determined only by the size distribution of the aerosol particles. For inhaled gases and vapors, deposition in the respiratory tract is assumed to depend entirely on the chemical form, which is consistent with results of studies indicating that regional deposition of a gas or vapor depends on its solubility and reactivity. 


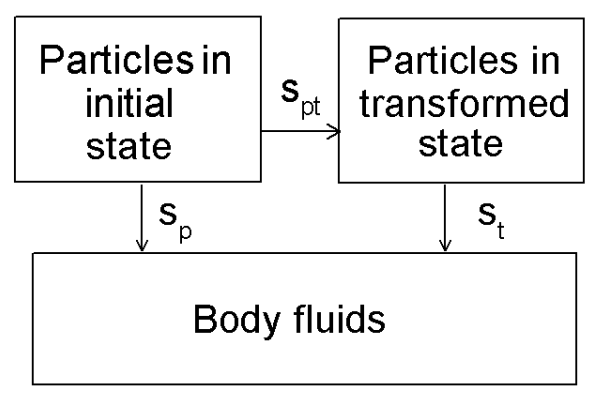

Figure 3.2. Schematic of treatment of time-dependent dissolution of inhaled particles in the HRTM (material deposited in a compartment is absorbed to blood at a rate $s_{p}$ and simultaneously transferred at a rate $s_{p t}$ to a parallel compartment representing a transformed state; transformed particles are absorbed to blood at a rate $s_{t}$ )

As a default approach, the HRTM assigns gases and vapors to one of three solubilityreactivity (SR) classes on the basis of the initial pattern of deposition in the respiratory tract. Class SR-0 represents insoluble and non-reactive gases or vapors, with deposition assumed to be negligible. Class SR-1 represents soluble or reactive gases or vapors, with deposition potentially occurring throughout the respiratory tract; the default assumption is $100 \%$ deposition, with $10 \%$ assigned to $\mathrm{ET}_{1}, 20 \%$ to $\mathrm{ET}_{2}, 10 \%$ to $\mathrm{BB}, 20 \%$ to bb, and $40 \%$ to AI. Class SR-2 represents highly soluble or reactive gases; in this case, $100 \%$ of inhaled activity is assumed to deposit in the extrathoracic airways $\left(\mathrm{ET}_{2}\right)$. If specific information is available for a gas or vapor, that information is used either to assign the material to one of the three solubility-reactivity classes or to develop material-specific parameter values.

\subsection{Basis for generic parameter values of the HRTM}

\subsubsection{Main types of information}

Except where otherwise indicated, the summaries of information on various parameter values are based on material given in ICRP Publication 66 or a recent ICRP (2002a) guidance document. However, the critical evaluations of the merits of the data are largely those of the authors of the present report.

Generic parameter values for the HRTM are based largely on data for human subjects (H1), either from controlled studies or follow-up studies of exposed workers. In some cases, gaps in the information are filled with data for laboratory animals (A1). H1 data underlying most parameter values of the HRTM are mainly for adult males. For some parameter values such as the ventilation rate, however, available information provides insight into variability among adult males and differences with age, gender, state of health, and other factors. 


\subsubsection{Ventilation rates}

There is considerable age- and gender-specific information on breathing rate and tidal volume (volume of inspired air) in individuals at rest or performing selected tasks of varying physical intensity. For any given age, gender, and level of activity, there is reasonable agreement in ventilation rates (tidal volume times breathing frequency) determined in different studies. While ethnic variability exists in lung volumes, flow rates, and tidal volume, minute ventilation at rest and at various levels of exercise does not appear to be very different between the ethnic groups that have been studied.

The average ventilation rate was estimated in ICRP Publication 66 (1994a) as a time-weighted average of ventilation rates for rest periods and periods of light and heavy activity. The least certain aspect of the calculation may be the characterization of a normal daily mix of activities. A normal mix of daily activities is more difficult to define for young children than for adults. The "time budgets" used in Publication 66 were based mainly on surveys conducted in the 1970s and 1980s and involving thousands of men, women, and children from a few different countries. Estimated average ventilation rates for adult males based on time budgets usually fall in the range 13-17 $\mathrm{L} \mathrm{min}^{-1}$.

Layton (1993) proposed a considerably different approach for estimating average ventilation rates based on the body's oxygen requirements. The rationale is that total air intake must be metabolically consistent with reasonably well established age- and gender-specific energy expenditure. Variability with age and gender as estimated by Layton is indicated in Figure 3.3. Layton's method yields 24-h average ventilation rates of about 10 (5.5-13) $\mathrm{L} \mathrm{min}^{-1}$ for adult males and 7 (5-9) $\mathrm{L} \mathrm{min}^{-1}$ for adult females, compared with the ICRP's values of $15.4 \mathrm{~L} \mathrm{~min}^{-1}$ and $12.6 \mathrm{~L} \mathrm{~min}^{-1}$, respectively. The HRTM method and the Layton method yield more consistent results for infants, children, and adolescents. For example, Layton derived the value 5.5 (3.5-7.5) $\mathrm{L} \mathrm{min}^{-1}$ for age $5 \mathrm{y}$, compared with the ICRP's value of $6.1 \mathrm{~L} \mathrm{~min}^{-1}$. The values in parentheses after Layton's central estimates indicate differences between relatively inactive and relative active subgroups of the population, rather than uncertainty in the central value.

\subsubsection{Total deposition in the respiratory tract}

Total deposition of inspired material in the respiratory tract has been studied extensively in human subjects. Most studies have involved adult Caucasian males and particle sizes of 1-10 $\mu \mathrm{m}$ aerodynamic diameter. While the sparseness of age- and gender specific data introduces some uncertainty into estimates for the general population, these uncertainties do not appear to be large for three reasons: (1) age- and gender-specific deposition can be estimated using a sophisticated theoretical deposition model based on well established physical principles; (2) there are some scattered age- and gender-specific data that allow spot checks of this model; and (3) the adult male respiratory tract appears to be a reasonably good surrogate in most respects for respiratory tracts in women and children. Gaps in human data on deposition of particles of aerodynamic diameter $<1 \mu \mathrm{m}$ have also 
been filled to some extent with theoretical models and with experiments involving physical models, particularly anatomically realistic hollow casts of nasal passages, naso-oropharynx, and larynx.

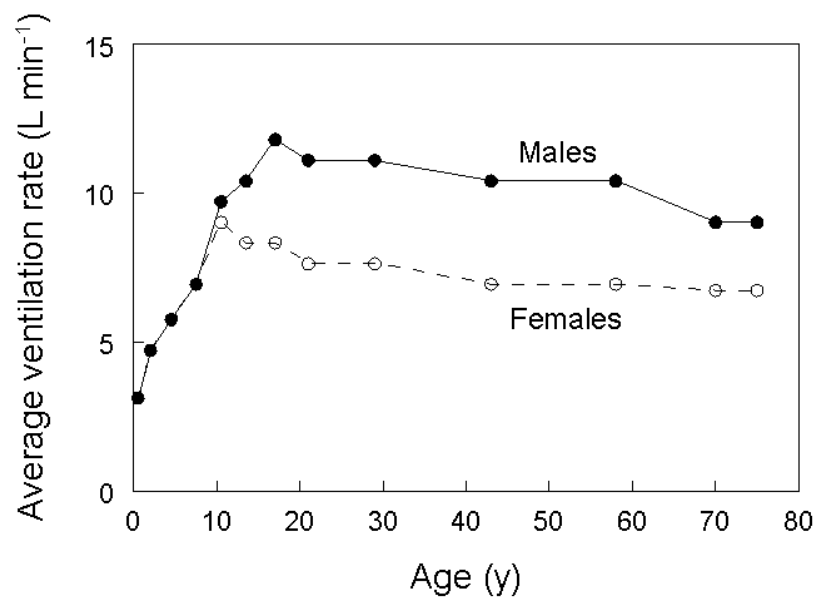

Figure 3.3. Variation with age and gender in 24-h average air intake, as estimated by Layton (1993) on the basis of the body's energy requirements

Because the filtration efficiencies of the nose and mouth are different, a subject's breathing habit, defined as the ratio of nose breathing to mouth breathing, affects the amount of inhaled material that deposits in the lungs. There is considerable variability in breathing habits, even among healthy persons of the same age and gender. Estimates of total deposition of inhaled particles generally are made by combining experimental data for mouth breathing with separately derived information for deposition in the extrathoracic regions.

\subsubsection{Initial division between the thoracic and extrathoracic regions}

Deposition in the extrathoracic region is generally estimated in parts, that is, as the sum of deposition fractions determined separately for the nose and oropharyngeal-laryngeal regions. Because processes that govern deposition of particles in the extrathoracic region depend strongly on particle size, different measurement techniques have been developed to measure different ranges of particle size. Particles with an aerodynamic diameter larger than about $0.5 \mu \mathrm{m}$ are deposited primarily by aerodynamic transport processes of inertial motion (impaction) and gravitational settling. For very large particles and fibers, interception with surfaces in the extrathoracic airways also contributes to deposition. Particles with an equivalent physical diameter less than a few tenths of a micrometer are deposited primarily by the thermodynamic transport process of Brownian diffusion.

Aerodynamic deposition in the nose is much better documented than aerodynamic deposition in other parts of the respiratory tract because this is the most easily observed 
portion of the tract. Measurement techniques include determination of the change in particle concentration when an aerosol is drawn in through the nose and out through the mouth during breath holding, external detection of deposited radioactivity after inhalation of labeled particles, and unfolding of the efficiencies of nasal deposition after measurement of the total respiratory tract deposition under different breathing maneuvers (nose breathing, pure mouth breathing, nose-in mouth-out, and mouth-in nose-out breathing). Most measurements have been for particle sizes of $1 \mu \mathrm{m}$ or greater. Perhaps the best available information on nasal deposition of smaller particles comes from measurements of deposition in anatomically accurate hollow casts of nasal passages, naso-oropharynx, and larynx. The casts were made from MRI scans of two adult males (one postmortem) and a 1.5-y-old child.

Most experimental studies of aerodynamic deposition in the oropharyngeal-laryngeal region have been performed with mouth breathing through a tube, since this is a convenient method for aerosol administration. Oral deposition is measured by repeated mouth washings directly after inhalation. The remainder of the extrathoracic deposition (oropharynx and larynx) is measured by external gamma counting. In some cases, activity in the stomach has also been measured within a short time of deposition and assumed to represent transfer of material from extrathoracic airways to stomach. The results of the studies are weakened by their apparent dependency on the structure and positioning of the mouthpiece and evidence that normal mouth breathing gives higher extrathoracic deposition than breathing through a mouthpiece. Also, determination of typical deposition in the oropharyngeal-laryngeal region is complicated by a large intersubject variability in extrathoracic deposition. This variability may be due mainly to differences in the morphology and physiology of the region, especially the larynx and vocal cords. Thus, the experimental results are not sufficient to model aerodynamic deposition in the mouth and lower extrathoracic regions with much accuracy. However, there is enough information to conclude that for particles less than about $10 \mu \mathrm{m}$ aerodynamic diameter, the bulk of the extrathoracic deposition during mouth breathing occurs in the larynx.

Deposition of submicron particles in the oropharyngeal-laryngeal airways has not been studied systematically in vivo in human subjects. As is the case for nasal deposition of small particles, reliance must be placed on particle deposition in hollow airway casts. Limited experiments using hollow casts indicate slightly higher deposition in the oropharyngeal-laryngeal airways than in the nasal passageway of the same individual, but there is some question concerning the anatomical realism in the hollow casts. For example, the positioning of the tongue during open-mouth breathing could influence deposition in ways not revealed by the hollow casts.

\subsubsection{Regional deposition in the lungs}

Although there have been several studies of regional deposition of material in the lungs of human subjects, the experimental data are not sufficiently complete or consistent to define the division between the tracheobronchial and alveolar regions in the adult male or the effects of different airway size in other groups. Estimates of regional deposition as a 
function of age and gender must be based mainly on theoretical models of the lungs. The theoretical model used in ICRP Publication 66 (1994a) evaluates the combined effects of convective and diffusive gas transport and aerosol loss processes within the airways of the lungs. Predictions of the theoretical model do not differ substantially from measured values of total lung deposition over a wide range of particle sizes.

The published experimental determinations of regional deposition in the respiratory tract are generally interpreted in terms of assumptions or models that may not be entirely correct. For example, the data as presented in the literature generally rely on the premise that particles deposited in distinct anatomical regions (e.g., the ciliated tracheobronchial airways or the respiratory airways of the alveolar interstitium) are cleared with distinctly different rates. In experimental studies involving human subjects, two distinct phases of particle clearance from the lungs are usually observed: an initial rapid phase succeeded by a slow phase with a fitted half-time of several tens of days. Results of some studies indicate, however, that a substantial fraction of particles deposited in the tracheobronchial region may have been cleared at a slow rate that was indistinguishable from clearance from the alveolar-interstitial airways. Thus, the fundamental premise of uniformly rapid ciliary clearance may not be valid.

There does not appear to be any information on regional lung deposition in children, but total thoracic deposition was measured in a group of children of average age about $10 \mathrm{y}$ and in another group of average age about $5 \mathrm{y}$. The data agree reasonably well with the theoretical deposition model, but only a small range of particle sizes was tested. Thus, total lung deposition is less well determined in children than in adults.

Variability of total or regional deposition of inhaled material in the respiratory tract in the population is not established. Predictions of the HRTM indicate little variation with age in deposition (Table 3.1), but in the case of regional deposition these predictions are based on assumptions and models rather than data.

Table 3.1. Variation with age in total and regional deposition fractions as a function of age, as predicted by HRTM for inhalation of $1-\mu \mathrm{m}$ particles (AMAD)

\begin{tabular}{cccccccc}
\hline & \multicolumn{7}{c}{ Age } \\
\cline { 2 - 7 } Region & $100 \mathrm{~d}$ & $1 \mathrm{y}$ & $5 \mathrm{y}$ & $10 \mathrm{y}$ & $15 \mathrm{y}$ & $\begin{array}{c}\text { Adult male } \\
\text { member of } \\
\text { public }\end{array}$ & $\begin{array}{c}\text { Adult } \\
\text { male } \\
\text { (worker) }\end{array}$ \\
\hline Al & 0.0856 & 0.0964 & 0.0985 & 0.0951 & 0.107 & 0.115 & 0.107 \\
Bbe & 0.0204 & 0.0171 & 0.0185 & 0.017 & 0.020 & 0.0195 & 0.0165 \\
BBi & 0.0104 & 0.0104 & 0.0104 & 0.0117 & 0.0169 & 0.0129 & 0.0124 \\
ET & 0.482 & 0.484 & 0.397 & 0.406 & 0.320 & 0.339 & 0.376 \\
Total & 0.598 & 0.608 & 0.524 & 0.530 & 0.464 & 0.486 & 0.512 \\
\hline
\end{tabular}




\subsubsection{Clearance of material from the lungs}

Particle clearance from the tracheobronchial region has been studied extensively. There is general agreement that mucociliary transport is the principal mechanism of particle removal from this region, the main flow is towards the pharynx, and mucous velocities decrease distally. There is much less agreement about the rate at which the decrease occurs or the precise pattern of mucous flow. Central values for tracheal mucociliary transport rates reported for different studies vary from about 4 to about $25 \mathrm{~mm} \mathrm{~min}^{-1}$. Measurements indicate that mucociliary clearance associated with deposition in the upper 8-10 airway generations is complete in a few hours. For the bronchioles, large discrepancies (factor of 40 or more) are seen in reported mucous velocities. Also, a relatively slow phase of clearance from the tracheobronchial region is indicated, but the fraction of the deposited amount associated with this phase and the rate of clearance are poorly understood.

There appears to be some retention in the airway walls in portions of the tracheobronchial region, based on studies of several species, including humans (ICRP, 1994a). Retention has been best quantified for the rat trachea, where about $0.7 \%$ of the deposit remained and was cleared with an estimated half-time of $88 \mathrm{~d}$. These results form the basis of the reference values describing retention in the tracheobronchial airway walls. Studies completed since the appearance of ICRP Publication 66 (1994a) and involving other animal species indicate that the HRTM may overestimate retention in the airway walls (M. Bailey, private communication).

Effects of different host factors or personal habits on lung mucociliary clearance have been investigated in a number of studies. Some permanent factors that can have a significant effect on lung mucociliary clearance are age (older persons may have slower clearance), possibly gender (women could have faster clearance), and cigarette smoking (slower clearance). There is no information on factors affecting either the magnitude or duration of slow tracheobronchial clearance, or of particle retention in the airways of humans.

Retention of insoluble particles in the alveolar region has been reasonably well characterized in controlled experimental studies covering periods up to $300 \mathrm{~d}$ after intake, at least in healthy adult males. Estimates of subsequent retention must be based on data for accidental intakes by human subjects or experimental intakes by laboratory animals. The data for accidental intakes by humans fall into two categories: in vivo measurements, usually of radioactive materials, over the thoracic region; and postmortem measurements of the amount of material in the lungs, which in some cases have been compared with the estimated intakes.

The experimental studies involving human subjects indicate that about $80 \%$ (range of central estimates, 56-93\%) of the initial alveolar deposit remains at $50 \mathrm{~d}$ after intake, $70 \%$ $(34-83 \%)$ at $100 \mathrm{~d}$, and $50 \%(11-64 \%)$ at $300 \mathrm{~d}$. This conclusion is based mainly on results of 10 experimental studies on human subjects. In four studies, both smokers and non-smokers were involved and were studied separately. The studies involve five 
different materials of differing solubility that would have tended to increase the variability compared to uniformly soluble materials.

Measurements of accidentally exposed persons at times remote from the primary exposure(s) indicate that $10 \%$ or more of deposited material may be retained in the lungs at 3000-10,000 d after exposure. The data are generally based on external measurements over the chest and, depending on the energy of the photon emission and knowledge of the biokinetics, may not clearly distinguish between activity in the lungs, lymph nodes, and thoracic bone. These external measurements serve mainly as upper-bound estimates on lung retention.

More direct evidence of very long-term retention of material in the lungs comes from postmortem measurements on occupationally exposed persons. For example, the distribution of actinides in former workers has been estimated on the basis of concentrations in tissues taken at autopsy, 30 years or more after exposure. The measurements have revealed that lung tissues may contain up to half of total-body activity, indicating a long retention time for a substantial portion of the inhaled material in the lungs. Much additional information on long-term retention of radioactivity in the lungs of workers, particularly plutonium workers in the U.S. and at the Mayak plant in Russia, has become available since the completion of the HRTM (e.g., Khokhryakov et al., 2005).

Evidence of long-term retention of inhaled material in the lungs also comes from studies on laboratory animals. For example, studies of inhaled $\mathrm{PuO}_{2}$ in dogs and baboons indicate that the half-time of particle clearance increases with time for years after intake and that several percent of the initial alveolar deposit may be retained in the lungs for many years after exposure.

\subsubsection{Retention in the lymph nodes}

Much information has been collected on retention of inhaled material in human lymph nodes. Interpretation of the data is complicated by uncertainties in the time-course and composition of the intakes, the high variability among lymph nodes of the same person, and the small portion of lymph nodes generally sampled. Nevertheless, the collective data provide an indication of the typical relative contents of lungs and lymph nodes at times remote from intake. Material is consistently found in both tissues, even many years after exposure has ceased.

Information on retention of inhaled material in human lymph nodes can be divided into four main categories: environmental exposure to nuclear weapons fallout, particularly plutonium and cerium; environmental exposure to natural long-lived radionuclides, particularly thorium; occupational exposure to actinides, particularly plutonium; and occupational exposure to mine dusts. Concentration ratios for tracheobronchial lymph nodes to lungs were 24 for cerium in non-occupationally exposed persons (one study); 1.9-15.0 (median 6.5) for plutonium in non-occupationally exposed persons (several studies); 1.5-20 (median 10.4) for thorium and uranium in non-occupationally exposed 
persons (several studies); 1.3-34.4 (median 9.7) for plutonium, thorium, uranium, and americium in occupationally exposed persons (several studies); 0.1-2.5 (median 1.8) for uranium in lungs of occupationally exposed persons (several studies). Relatively low concentrations were determined for dust concentrations in hilar lymph nodes of coal miners (two studies).

\subsubsection{Absorption to blood}

Absorption of inhaled material from the respiratory tract to blood is viewed as a two-stage process: (1) dissociation of the particles, and (2) uptake of the dissociated material. Absorption rates tend to change with time because the process of dissociation changes the physicochemical form of the material. There is insufficient information to model the factors involved in dissolution and uptake, in order to predict the rate of absorption into blood for specific materials.

Theoretically, absorption to blood can be estimated as the difference between lung retention and particle transport from the lungs. This method assumes that particle transport is not the dominant mechanism of removal from lungs. Particle transport is often the observed dominant mechanism for very insoluble particles. It is also often the dominant mechanism in studies with small rodents (rats, hamsters, mice), which have relatively high particle transport rates from the alveolar region.

In practice, estimates of absorption into blood are usually based on observations of urinary excretion or accumulation of material in systemic organs. This approach requires a reasonably good systemic biokinetic model and information on gastrointestinal uptake. If such observations are not available for human subjects, it is preferable to use data for medium to large animal species, such as baboons or dogs.

The absorption rate of a given compound may vary with such factors as particle size, method and temperature of formation, isotopic composition, and presence of other metals. Particles encountered in practice may be complex mixtures of ill-defined compounds, and the radionuclide of concern may be present as a minor constituent of the material. The first stage in absorption, particle dissolution, is assumed to be determined by the particle matrix, rather than by the radionuclide itself.

Uptake of dissolved material to blood is thought to be rapid for most elements. In some cases, however, a significant fraction of the dissociated material is absorbed slowly as a result of binding to respiratory tract components. For example, such binding has been observed for cadmium.

Even with relatively insoluble materials, a rapid phase of uptake to blood is often observed immediately after inhalation. This may be due to some combination of the following factors: a soluble component of the inhaled material, an unstable particle surface, presence of a portion of the activity in small particles with a relatively high specific surface area, particles small enough to pass directly to blood, and transformation of the deposited material into a form that is less readily absorbed. As discussed in a later 
section, however, the rapid phase of absorption may be absent for some materials, and the default absorption types used with the HRTM do not take this possibility into account.

Dissolution rates in the respiratory tract may differ according to whether the particles are in the liquid lining the epithelium, or phagocytosed by macrophages. The phagosomes have a relatively low $\mathrm{pH}$ (4-5), but $\mathrm{pH}$ alone is not sufficient to account for observed differences in dissolution rates in alveolar macrophages and saline.

Several simplifying assumptions were made in the development of the HRTM to model clearance kinetics for the wide range of materials considered by the ICRP:

(1) It is assumed that clearance kinetics may be expressed in terms of fractional clearance rates. This same assumption is made in most biokinetic models used by the ICRP.

(2) Clearance rates due to particle transport and absorption are assumed to be independent. The limited supporting evidence for this assumption includes two interspecies comparisons involving inhalation of monodisperse ${ }^{57} \mathrm{Co}$-labeled cobaltic oxide. The first study involved human volunteers, baboons, dogs, guinea pigs, hamsters, and three strains of rats. The second study involved baboons, dogs, and one strain of rats. Particle sizes were 0.8-1.7 $\mu \mathrm{m}$. The pattern of particle transport rates over time in the various species was qualitatively different from that of the absorption rates. Initial absorption rates for the $0.8 \mu \mathrm{m}$ particles were higher than those of 0.9 and $1.7 \mu \mathrm{m}$ particle sizes in each species, whereas there was no apparent effect of particle size on particle transport rates. During the first three months, the absorption rate remained constant or initially increased with time in each group, while the particle transport rate decreased monotonically. There was no correlation between the interspecies differences in absorption and particle transport rates. Both rates were high in rats and low in humans and baboons. Dogs showed rapid absorption of cobalt to blood but very slow particle transport.

(3) Particle transport rates are assumed to be the same for all materials. This assumption is not strongly supported by available data but seems reasonable in lieu of material-specific data, at least for mucociliary transport. Less is known about factors influencing particle transport from the AI region to the bb region.

(4) The rate of absorption of a material to blood is assumed to be the same in all regions of the respiratory tract, including the regional lymph nodes. This is an assumption of convenience that may be particularly inaccurate for the time immediately after deposition, when the rate of absorption of dissolved material might be higher than average in the alveolar region where the air-blood barrier is thinnest. In a study involving 12 different drugs with widely varying properties such as molecular size, lipid solubility, degree of ionization, and chemical structure, absorption to blood was about twice as rapid following administration by inhalation than following intratracheal injection (Schanker et al., 1986). It is possible that absorption was substantially more rapid from the alveolar region than from the 
tracheobronchial region. The assumption of a uniform absorption rate in the respiratory tract may be more reasonable at times later than a few hours after inhalation, when most particles are likely to be within macrophages in all regions of the lungs, and dissolution in macrophages might be the rate-determining step in the absorption of activity into blood.

(5) It is assumed that material deposited in the anterior nasal passage is not absorbed to blood. This is based on the structure of the epithelium; the structure is much like that of skin, which shows low (but not zero) permeability by most materials.

\subsubsection{Definition of lung dose}

Lung dose is defined in ICRP Publication 66 as a weighted average of the calculated doses to three radiosensitive regions of the lungs: the bronchial region (BB), the bronchiolar region (bb), and the alveolar-interstitial (AI) region. The ICRP recommends the use of equal weights, or detriment apportionment factors, for these three regions but points out the possibility, in view of the regional distribution of spontaneous lung cancers in the general non-smoking population, that uniform irradiation of the lungs may be more likely to lead to the induction of cancer in the bronchial region than in the alveolar and bronchiolar regions.

Estimates of lung dose are relatively insensitive to the choice of regional apportionment factors for radionuclides that emit penetrating radiations because the three regions are in close proximity to one another and there is cross-irradiation from activity within these regions. On the other hand, dose estimates may depend strongly on the choice of regional apportionment factors for some radionuclides with relatively non-penetrating radiations, i.e., radionuclides that emit mainly alpha or low-energy beta particles or discrete electrons.

\subsection{Existing evaluations of potential errors and uncertainties in HRTM components and predictions}

\subsubsection{Expert judgments by the developers of the HRTM}

ICRP Publication 66 (1994a) gives brief summaries of uncertainties associated with many of the parameter values of the HRTM, representing the expert judgments of the ICRP task group that developed the model. For example, it is pointed out that reference values describing particle clearance

"are based on indirect information and therefore involve a considerable amount of judgment. Some rates are based entirely on animal studies, and extrapolating results to humans involves a possible systematic error, which could be large. For example, the alveolar clearance rate at $100 \mathrm{~d}$ after inhalation in humans is about 5 times greater than in dogs, and 5 times lower than in mice... Maximum mucociliary transport rates in the trachea ranged from $2 \mathrm{~mm} \mathrm{~min}^{-1}$ in rats to $10 \mathrm{~mm} \mathrm{~min}^{-1}$ in dogs. These are all, however, within a factor of 2.5 of the [reference value for humans] (5 
$\left.\mathrm{mm} \min ^{-1}\right) \ldots$ Thus the combination of a high degree of intersubject variation and possible systematic errors means that the uncertainties in the reference values are large and difficult to quantify. It is ... assumed that the uncertainty in the reference value is lognormally distributed and ...that there is a $95 \%$ probability that the true mean value lies within a factor of $\Phi_{u}$ of the true value..."

The HRTM Task Group concluded that the two examples of interspecies differences suggest that a factor of 3 is reasonable whenever a rate is based entirely on animal data. With regard to estimated rates of clearance from the alveolar region, which are based mainly on data for human subjects, they indicate that the mean clearance rate up to $100 \mathrm{~d}$ may be known within about 20\%, the rate at 200-300 d within a factor of 2 , and the long-term removal rate from the AI region to the bronchioles $\left(0.0001 \mathrm{~d}^{-1}\right.$, corresponding to a half-time of almost $7000 \mathrm{~d}$ ) within a factor of 3. They do not address uncertainties associated with model structure, e.g., the use of three retention components to describe behavior of material in the deep lungs.

\subsubsection{Expert judgments by international experts}

In the mid-1990s a joint project was conducted by the NRC and the Commission of the European Communities (CEC) for the purpose of developing traceable uncertainty distributions for input variables to their probabilistic accident consequence codes (NRCCEC, 1997). A procedure was devised to elicit expert judgment regarding uncertainties in input to that code. Elicitation questions were developed, tested, and clarified. Internationally recognized experts were selected. Probability training exercises were conducted to establish ground rules for the experts' preparation of material and for the formal elicitation process. Experts developed their uncertainty distributions and rationales independently. A meeting was held in which the experts discussed their independent conclusions and rationales and were given the opportunity to change their conclusions before final, private solicitation interviews with individual experts.

Several of the elicitation questions were directly related to parameters of the HRTM, e.g., ventilation rates, deposition fractions in different regions of the respiratory tract, and retention in different regions of the tract at different times. The aggregated results generally gave surprisingly wide confidence intervals (5-95\%), perhaps reflecting the fact that the participants were actually experts in a variety of different areas related to the radiation dosimetry and had much different levels of familiarity with behavior and dosimetry of inhaled radionuclides. That is, the uncertainties expressed by several of the participants may have reflected their understanding of information partly outside their real areas of expertise, rather than the true state of knowledge of the behavior of inhaled radionuclides.

\subsubsection{Parameter uncertainty analysis applied to ${ }^{222} \mathrm{Rn}$ daughter dosimetry}

Marsh et al. (2002) applied standard methods of parameter uncertainty analysis to examine the uncertainty in dose to an adult from inhalation of ${ }^{222} \mathrm{Rn}$ progeny in the home. This was a slight variation of an earlier analysis by Birchall and James (1994) of the 
uncertainty in dose from inhalation of ${ }^{222} \mathrm{Rn}$ progeny by a miner. The main purpose was to examine whether uncertainties in selected parameter values of the HRTM as applied to

${ }^{222} \mathrm{Rn}$ progeny could explain the discrepancy between the epidemiologically based estimate and the roughly three-fold greater HRTM-derived estimate of risk associated with unit exposure to radon daughters. It was assumed that the HRTM is a realistic representation of the physical and biological processes, and that the parameter values are uncertain. Uncertainties associated with the ICRP's radiation weighting factor for alpha particles (20) were not considered, and the ICRP's tissue weighting factor for the lung (0.12) was left fixed. The investigators concluded that the uncertainties in the HRTM parameters, at least those that were considered in these two analyses, cannot totally account for the discrepancy between the dosimetric and epidemiological approaches.

The rationale behind the analyses by Marsh, Birchall, and coworkers is not clear. The discrepancies between HRTM predictions and epidemiological data seem modest in view of the sizable uncertainties in the miner data and in components of the HRTM-based dose estimates not addressed in the analysis. For example, the actual intakes of ${ }^{222} \mathrm{Rn}$ progeny by uranium miners through about 1967 were based on sparse and often biased information (e.g., company records) and in many cases could be in error by an order of magnitude. An example of uncertain input to dose estimates not addressed by Marsh, Birchall, and coworkers is the radiation weighting factor of 20 for alpha particles. Also, the investigators may have overstated current understanding of the biokinetics of ${ }^{222} \mathrm{Rn}$ daughters in the respiratory tract, such as the time course of absorption of ${ }^{214} \mathrm{~Pb}$ and ${ }^{214} \mathrm{Bi}$ to blood.

\subsubsection{Detailed parameter uncertainty analysis for inhaled $\mathrm{PuO}_{2}$ or $\mathrm{UO}_{2} / \mathrm{U}_{3} \mathrm{O}_{8}$}

Bolch and coworkers (Bolch et al., 2001, 2003; Farfan et al., 2003; Huston et al., 2003) published a series of papers addressing uncertainties associated with predictions of the HRTM as discerned from parameter uncertainty analysis. They reviewed the original data on which 69 default parameter values of the HRTM were based, selected subjective distributions that they referred to as "probability distributions" for these parameter values, and propagated these distributions through the HRTM to derive distributions of lung dose. Uncertainties in the following model predictions were addressed in separate papers: particle deposition (Bolch et al., 2001); particle clearance for the specific cases of inhaled ${ }^{239} \mathrm{PuO}_{2}$ or ${ }^{238} \mathrm{UO}_{2} /{ }^{238} \mathrm{U}_{3} \mathrm{O}_{8}$ (Bolch et al., 2003); regional tissue doses for inhaled ${ }^{239} \mathrm{PuO}_{2}$ or ${ }^{238} \mathrm{UO}_{2}{ }^{238} \mathrm{U}_{3} \mathrm{O}_{8}$ (Farfan et al., 2003); and identification of the input parameters to which their dose estimates for inhaled ${ }^{239} \mathrm{PuO}_{2}$ were most sensitive (Huston et al., 2003). Conclusions of Bolch and coworkers regarding the level of uncertainty in lung dose from inhaled ${ }^{239} \mathrm{PuO}_{2}$ or ${ }^{238} \mathrm{UO}_{2} /{ }^{238} \mathrm{U}_{3} \mathrm{O}_{8}$ varied markedly with particle size (Farfan et al., 2003). For "typical exposure scenarios" involving these radionuclides, the ratio of their derived $95 \%$ to $5 \%$ confidence levels was on the order of 8-10 for particles diameters $0.1-1.0 \mu \mathrm{m}$. They concluded that the uncertainty in lung dose increases by factors of 50 or more over the particle size range from 0.1 to $20 \mu \mathrm{m}$.

In the papers by Bolch et al., the term "uncertainty" refers to "both (1) a lack of knowledge of the model parameters, and (2) any biological variability these parameters 
would exhibit within the population of interest" (Bolch et al., 2001). This suggests that the results should be interpreted as reflecting the investigators' uncertainty in a model estimate for a healthy individual, based on the assumption that model parameter values represent the only sources of uncertainty. This differs from the use of the term "uncertainty" in the present report, which refers to a confidence level regarding a central value for a population.

For the case of inhaled ${ }^{239} \mathrm{PuO}_{2}$, Huston et al. (2003) concluded that the following parameter values dominated the dose estimate: the ventilation rate, fractional deposition in the AI region, the clearance rate constant for slow-phase absorption, and a clearance rate constant for particle transport from one of the AI compartments. This result cannot be carried over to inhalation of radionuclides in general because the dominant parameters will depend on factors such as half-life of the radionuclide, mode of decay, energies of emitted radiations, particle size, and solubility.

\subsubsection{A modification of the analysis of Bolch and coworkers for inhaled $\mathrm{PuO}_{2}$}

Aden and Scott (2003) modified the analysis by Huston et al. (2003) for inhaled $\mathrm{PuO}_{2}$ in an effort to improve the characterization of uncertainty in dose per unit intake as applied to a hypothetical population of nuclear workers engaged in light work-related exercise. Their parameter uncertainty distributions were similar to those selected by Huston and coworkers, with the main exception that polydisperse rather than monodisperse particles were considered. Aden and Scott concluded that the HRTM overestimates deposition for most of the adult male population when aerodynamic particle sizes are greater than 10 $\mu \mathrm{m}$, especially for deposition in bb and AI.

\subsubsection{Comparison with an alternate ventilation model}

As discussed earlier, the ICRP modeled the ventilation rate by applying "time budgets" to ventilation rates observed in human subjects at different levels of activity. Layton (1993) developed an alternate, independent model of ventilation based on energy requirements that is at odds with the ICRP model, particularly for adults (Figure 3.4).

Layton's ventilation model also involves uncertainties, but the potential underestimate does not seem large enough to explain the discrepancies between that model and the HRTM. Layton's estimates are derived using the equation $\mathrm{VE}=\mathrm{E} \times \mathrm{H} \times \mathrm{VQ}$, where $\mathrm{VE}$ is the ventilation rate $\left(\mathrm{L} \mathrm{min}^{-1}\right), \mathrm{E}$ is the average rate of energy expenditure (kilojoules $\left.\min ^{-1}\right), \mathrm{H}$ is the volume of oxygen (at standard temperature and pressure) consumed in the production of 1 kilojoule of energy, and VQ is the ratio of ventilation rate to oxygen uptake rate. The value $\mathrm{H}$ has been determined within narrow bounds. Comparisons of different food consumption surveys for the U.S. population give reasonably consistent results, when biases in the data are taken into account as was done by Layton. The largest potential error in the method may lie in the parameter VQ. Layton concluded that VQ is nearly independent of the ventilation rate and proposed the value VQ $=27$ for all ages and activity levels and for both genders. This value is based on data for adult humans (almost all male subjects, a large portion of which were highly trained athletes) and data 
from two studies on newborns. Little information is available for adult females, but results of a study on children of age 7-17 y (Zapletal et al., 1987) give a mean value for VQ of about 36 and suggest a slight increase with age, from about 35 at age $7 \mathrm{y}$ to about 37 at age $17 \mathrm{y}$.

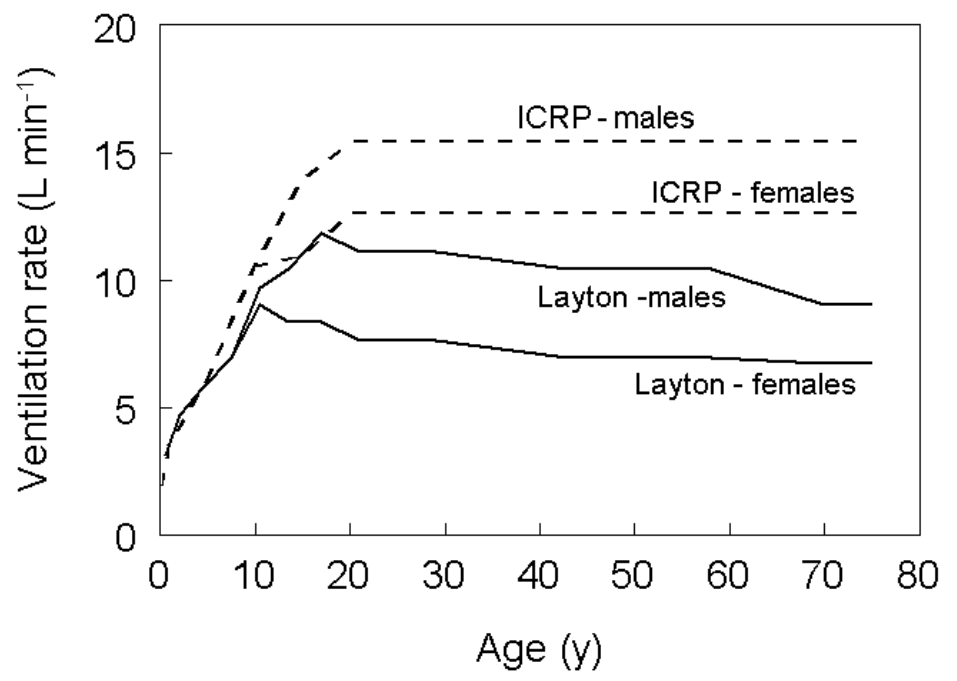

Figure 3.4. Comparison of predictions of the ventilation submodel of the HRTM with values derived from an independent model based on energy requirement (Layton, 1993)

Thus, the Layton model involves some uncertainties, but it seems at least as defensible as the ICRP model of ventilation. The discrepancies between the two models indicate a larger uncertainty in the ICRP's ventilation model than might be assigned by assessing the data underlying the parameters of the ICRP model. This illustrates how comparison of alternate models can provide insight into potential modeling errors that may not be revealed with parameter uncertainty analysis.

\subsubsection{Comparison with alternate parameter values for inhaled mercury vapor}

This example is concerned with potential errors in a specific application of the HRTM, in this case to inhaled mercury vapor, rather than in generic features of the HRTM. The ICRP's application of the HRTM to mercury vapor is compared with an alternate application based on revised parameter values developed from a critical review of the literature.

The ICRP's model for inhaled mercury vapor was introduced in ICRP Publication 30, Part 2 (1980) but later recast in the context of the HRTM (ICRP Publication 68, 1994b). According to the original and updated versions of the model, $70 \%$ of inhaled mercury vapor is deposited in the lungs, and all of the deposited mercury is absorbed to blood with 
a half-time of $1.7 \mathrm{~d}$. In the original version, mercury vapor is assumed, in effect, to be distributed uniformly in the lungs. In the updated version applied in ICRP Publication 68 (ICRP, 1994b), 10\% is assigned to the bound compartment of the large bronchi (BB), $20 \%$ to the bound compartment of the small bronchi (bb), and $40 \%$ to the bound compartment of the alveolar-interstitial region (AI). The bound compartments represent deeply penetrated material assumed not to be subject to removal by particle transport processes but cleared only to blood.

A critical review of the literature on the biokinetics of inhaled mercury vapor was performed recently as part of an accident analysis for the Spallation Neutron Source (SNS) at ORNL (Leggett et al., 2001). The SNS uses stable mercury as a target. Radioisotopes of mercury build up during irradiation of the target by neutrons and high energy protons and could be released as vapor during an accident, particularly a fire. It was concluded from the review that the ICRP's model for mercury vapor does not accurately represent current information, or even information available at the time the parameter values were developed for use in ICRP Publication 30. The evidence indicates that inhaled mercury vapor deposits largely in the AI region, most of the deposited mercury is nearly instantaneously absorbed to blood, and the remainder may have multiple components of retention in the lungs

Parameter values of the HRTM were modified for agreement with reevaluated and updated bioassay data (Leggett et al., 2001). In the modified version:

1. Total retention in the respiratory tract is increased from $70 \%$ to $80 \%$.

2. $2 \%$ of inhaled activity is assigned to the extrathoracic region $\left(\mathrm{ET}_{2}\right) 1 \%$ to the large bronchi (BB), $2 \%$ to small bronchi (bb), $75 \%$ to AI, and $20 \%$ is exhaled. As in the ICRP model, mercury atoms are assumed to be instantaneously transferred to the "bound" compartments in each region.

3. A portion of activity deposited in AI, equivalent to $70 \%$ of the deposition in the respiratory tract (that is, $0.7 \times 80 \%=56 \%$ of inhaled activity), is absorbed to blood with a half-time of $1 \mathrm{~min} ; 80 \%$ of the remaining activity in all regions is absorbed with a half-time of $8 \mathrm{~h}$; and $20 \%$ is absorbed with a half-time of $5 \mathrm{~d}$.

Dose estimates based on the ICRP model and the proposed revision are compared in Table 3.2. In each case, the ICRP's current systemic biokinetic model for mercury was used.

The revised model reduces the effective dose coefficients for ${ }^{203} \mathrm{Hg}$ and ${ }^{197} \mathrm{Hg}$ by factors of 4 and 10, respectively, due to the decreased mean residence time of these short-lived isotopes in the lungs. The effective dose coefficient is increased slightly for the longerlived isotope ${ }^{194} \mathrm{Hg}$, for which a small, long-term component of retention in the updated model becomes a more important factor. 


\begin{tabular}{|c|c|c|c|c|}
\hline \multicolumn{5}{|c|}{$\begin{array}{l}\text { Table 3.2. Comparison of dose coefficients for inhalation of mercury } \\
\text { vapor by a worker, based on the HRTM with current ICRP parameter } \\
\text { values for mercury vapor (ICRP, 1994b) and parameter values } \\
\text { proposed by Leggett et al. (2001) }\end{array}$} \\
\hline \multirow{2}{*}{ Mercury isotope } & \multirow{2}{*}{ Half-life } & \multicolumn{3}{|c|}{$\begin{array}{l}\text { Ratio of dose coefficients B:A based on } \\
\text { HRTM with (A) current and (B) proposed } \\
\text { parameter values }\end{array}$} \\
\hline & & Lung & Kidneys & Effective dose \\
\hline $\mathrm{Hg}-203$ & $46.6 \mathrm{~d}$ & 0.09 & 1.2 & 0.26 \\
\hline $\mathrm{Hg}-197$ & $64.1 \mathrm{~h}$ & 0.06 & 1.7 & 0.10 \\
\hline $\mathrm{Hg}-194$ & $260 y$ & 0.74 & 1.1 & 1.1 \\
\hline
\end{tabular}

\subsubsection{Comparison with the NCRP respiratory model}

At the time of development of the HRTM by the ICRP, a committee of the NCRP was also developing a respiratory tract model (NCRP, 1997). The ICRP and NCRP models are compared in NCRP Commentary No. 15 (1998) on the reliability of models used in radiation dose assessment, where it is pointed out that the differences in these two models offer a unique opportunity for assessing uncertainties about lung retention and dosimetry.

The HRTM Task Group and NCRP committee considered essentially the same information but chose different model structures, made different assumptions, and sometimes emphasized different parts of the database. According to the authors of NCRP Commentary No. 15, predictions of the two models are generally consistent where information is fairly clear but make different predictions where evidence is ambiguous or missing. With regard to retention and uptake of activity inhaled in insoluble form, the HRTM and NCRP model both move away from the lung model of ICRP Publication 30 in the same general direction. For example, both models predict substantially lower absorption from relatively insoluble materials than is predicted by the lung model of ICRP Publication 30. Predictions of total deposition are reasonably consistent, but noticeable differences in predictions are seen for regional depositions, particularly in the extrathoracic and tracheobronchial regions. Comparisons are complicated by the fact that they are scenario dependent. For example, regional depositions of inhaled material were compared using two different scenarios, one based on the ICRP's default parameter values for breathing and aerosols and one based on corresponding parameter values taken from the NCRP report. Good agreement between the ICRP and NCRP models was obtained in model predictions of deposition in all regions of the respiratory tract when the NCRP scenario was used. When the ICRP scenario was used, good agreement was found for the alveolar-interstitial region, moderately good agreement (difference of 32\%) was obtained for the tracheobronchial region, and a difference of $76 \%$ was observed for the extrathoracic region.

We agree with the conclusion in NCRP Commentary No. 15 that comparison of the ICRP and NCRP respiratory models offers a unique opportunity for assessing uncertainties 
about lung retention and dosimetry. We believe that a detailed comparison of these two models should be performed as one of the initial tasks in a comprehensive assessment of potential errors in dose estimates for inhaled radionuclides.

\subsubsection{Comparison with independent data}

This section illustrates potential errors in the application of default absorption types used with the HRTM. It is well known, of course, that the default absorption types may not provide close fits to data for specific materials, and the ICRP recommends that materialspecific parameter values be developed when information allows. Case studies involving inhalation of certain forms of enriched $\mathrm{U}$ or ${ }^{238} \mathrm{Pu}$ show that the general pattern of monotonically declining dissolution rates depicted by all three default absorption types can differ radically from the real situation.

In the late 1960s a group of workers was exposed over several months to high concentrations of airborne uranium aluminide $\left(\mathrm{UAl}_{\mathrm{x}}\right)$, a material used in fuels for research and test reactors. Exposure to $\mathrm{UAl}_{\mathrm{x}}$ occurred mainly in a work area where $\mathrm{UAl}_{\mathrm{x}}$ powder was formed and pressed into a compact to be used in the core of a fuel plate. Monitoring of urinary data over the first few months of the program did not indicate unusually high exposures to uranium. Several months after the start of the $\mathrm{UAl}_{\mathrm{x}}$ fuel fabrication program, however, it became evident that urinary uranium lagged far behind $\mathrm{UAl}_{\mathrm{x}}$ intake. For example, in workers who had been removed from exposure, the rate of urinary excretion of uranium continued to rise over a period of months, reached a peak, and then decreased sharply (Figure 3.5). High lung burdens in several workers had resulted before the problem was recognized. Retrospective estimates of lung dose based on revised parameter values for the ICRP respiratory model (based on intake and excretion data for the aluminide workers) were in the range $0.5-2 \mathrm{~Sv}$ for 18 of the workers and $3.5 \mathrm{~Sv}$ for the most highly exposed worker.

As illustrated in Figure 3.5 for Type $\mathrm{M}$ and Type $\mathrm{S}$ aerosols, the urinary data are inconsistent with the ICRP's default absorption types for inhaled particulates. Each of the three sets of default parameter values (F, M, or S) depicts a monotonically decreasing rate of dissolution and absorption to blood following acute intake. When combined with the ICRP's systemic biokinetic models for specific elements, the modeled pattern of dissolution and absorption is reflected in the urinary excretion rate over time. That is, the ICRP models with default parameter values predict a decreasing rate of urinary excretion as a function of time following acute intake, or a rise and fall in the excretion rate reflecting changing exposure levels during chronic exposure.

The authors developed parameter values for the HRTM to describe the behavior of $\mathrm{UAl}_{\mathrm{x}}$ seen in these workers, using parameter values for Type $\mathrm{S}$ as a starting point. The parameter values that were adjusted included those representing the dissolution rate as well as those representing mechanical clearance rates from the AI region. While it was the intention of the HRTM Task Group that the parameters $s_{p}, s_{p t}$, and $s_{t}$ (Figure 3.2) be modified to fit material-specific information, they considered the particle clearance rates to be fixed. The assumption that the particle clearance rates from AI are the same for the 
initially deposited material and transformed material appears to be a convenient assumption with little or no physiological basis.

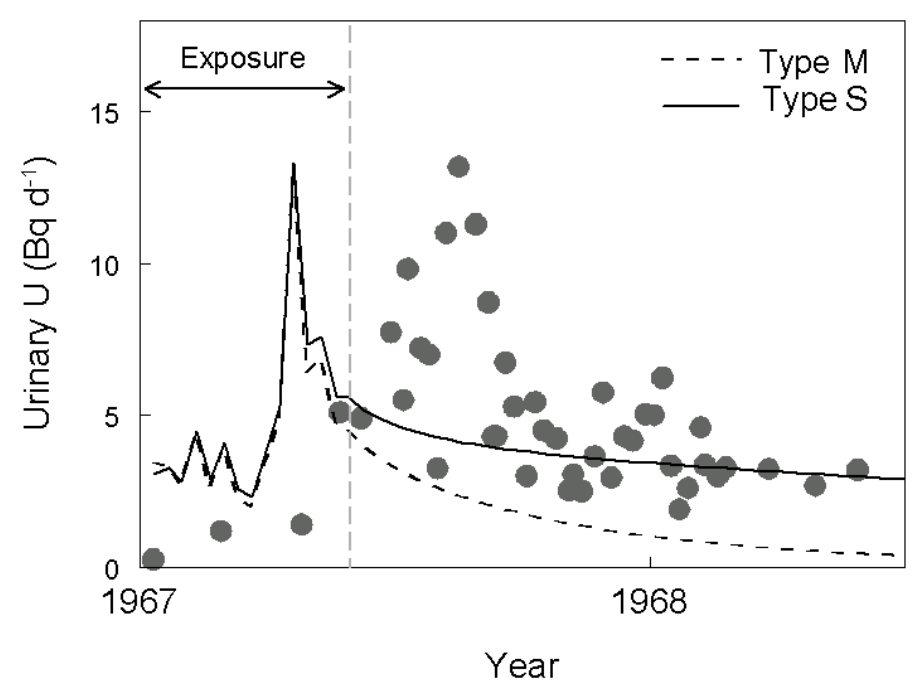

Figure 3.5. Urinary excretion data for a worker exposed to airborne $\mathrm{UAl}_{\mathrm{x}}$, compared with predictions of the HRTM based on Type $M$ or Type $S$ aerosols; rise and fall of the model predictions during the exposure period reflect the change in measured air concentrations of $\mathrm{UAl}_{\mathrm{x}}$ during that period

The fit of the HRTM with parameter values adjusted by the present authors to account for a different dissolution kinetics model is illustrated in Figure 3.6 for one of the most heavily exposed workers.

As illustrated in Table 3.3, dose coefficients for inhalation of ${ }^{234} U$ based on the $\mathrm{UAl}_{\mathrm{x}}-$ specific parameter values differ substantially from those assuming Type $M$ or Type $S$ for some tissues. However, the effective dose for all three cases is largely determined by the lung dose, which does not differ greatly for these cases. The effective dose for "Type $\mathrm{UAl}_{\mathrm{x}}$ " is slightly less than that for Type $\mathrm{S}$ and about 2.5 times that for Type M.

Particularly large uncertainties in predictions of the HRTM with default parameter values arise in dose reconstructions based on urinary data. Table 3.4 compares back calculations of effective dose based on parameter values for Type $\mathrm{M}$ or Type $\mathrm{S}$ with calculations based on the $\mathrm{UAl}_{\mathrm{x}}$ specific parameter values developed by the authors (labeled as ORNL estimates). The comparisons are made for acute inhalation of $\mathrm{UAl}_{\mathrm{x}}$, assuming that a single urinary $U$ measurement is available at $7 \mathrm{~d}, 15 \mathrm{~d}, 30 \mathrm{~d}$, or $90 \mathrm{~d}$ after intake. Of course, there are uncertainties in the dose estimates based on ORNL's material-specific parameter values. Nevertheless, the material-specific parameter values are consistent with a large amount of urinary and fecal data for these workers. 


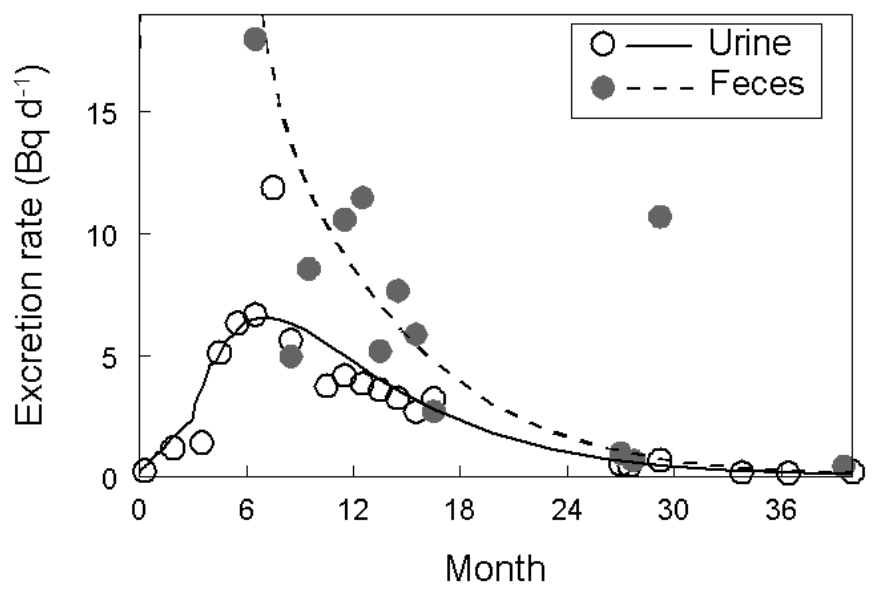

Figure 3.6. Comparison of the predictions of the modified HRTM with urinary $U$ data for a worker who was heavily exposed to $\mathrm{UAl}_{\mathbf{x}}$

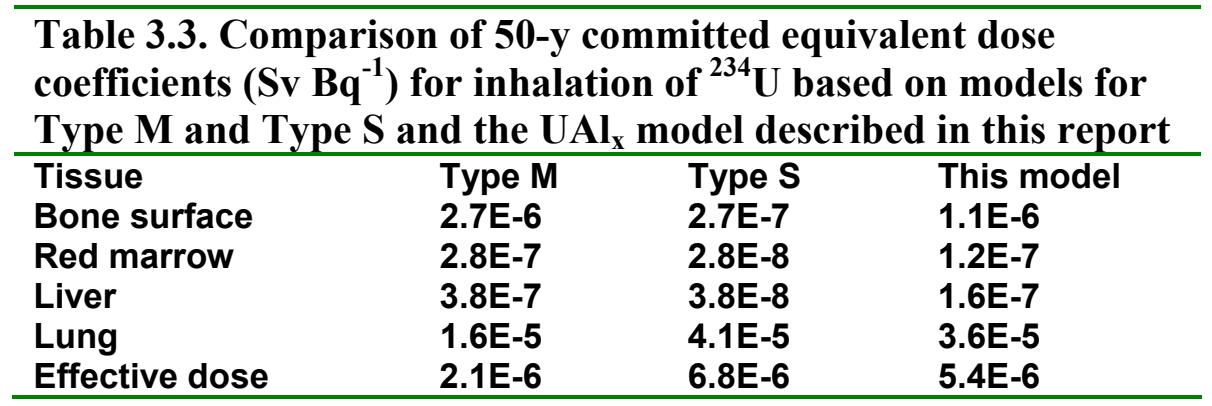

A review of the literature revealed that the general pattern of delayed excretion seen for the workers exposed to $\mathrm{UAl}_{\mathrm{x}}$ has been observed for several actinide elements including plutonium, americium, and californium (Healy, 1957; King, 1980; Wood and Sheehan, 1971; Rundo and Sedlet, 1973). For example, Wood and Sheehan (1971) reported follow-up measurements on five workers exposed to an accidental release of ${ }^{238} \mathrm{Pu}$ oxide. The assay of initial urine samples indicated some uptake of plutonium but did not indicate, on the basis of the monotonically declining model predictions available at the time, that any of the intakes had exceeded $10 \%$ of the limit in place at the time. The rate of excretion of plutonium in urine continued to increase over a period of months in all five subjects. In each of the subjects, urinary excretion rates peaked about 6-8 mo after the incident and then declined with a half-time of about 8 mo. It belatedly became apparent that intake limits had been exceeded in some workers. 


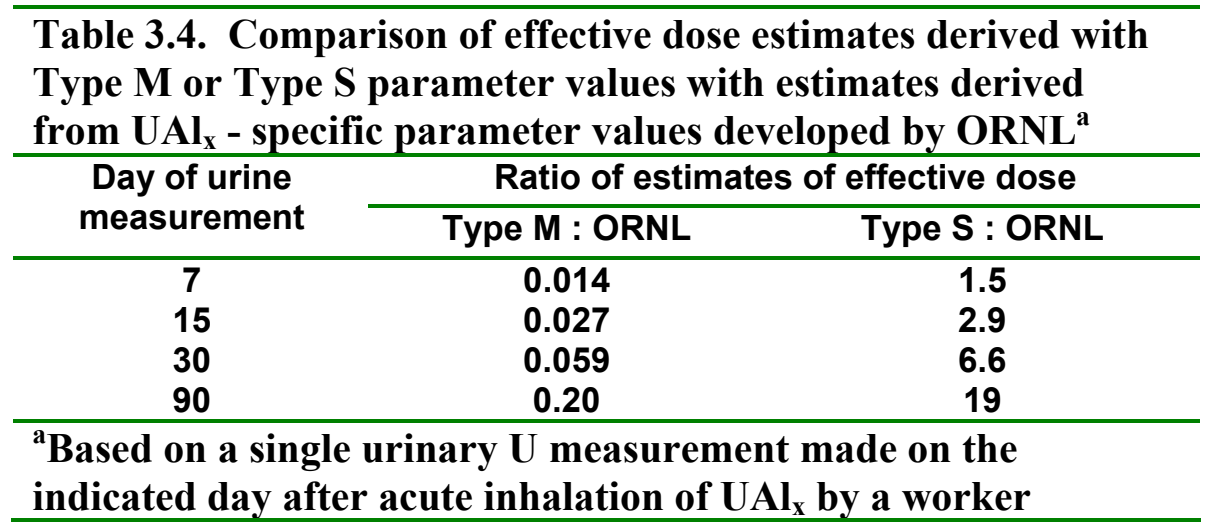

In addition, on the basis of a study of the fate of inhaled ${ }^{238} \mathrm{PuO}_{2}$ in beagle dogs, Mewhinney and Diel (1983) concluded that eventual fragmentation of ${ }^{238} \mathrm{PuO}_{2}$ particles deposited in the lung leads to enhanced dissolution, translocation and excretion of ${ }^{238} \mathrm{Pu}$ compared with ${ }^{239} \mathrm{PuO}_{2}$ as measured in previous studies. Mewhinney and Diel listed several inferences that may be drawn from their results in evaluations of cases of human inhalation of ${ }^{238} \mathrm{PuO}_{2}$ : (1) During the first 2-3 months after exposure the behavior of inhaled ${ }^{238} \mathrm{PuO}_{2}$ in the lungs is expected to be similar to the reasonably well established behavior of inhaled ${ }^{239} \mathrm{PuO}_{2}$. (2) At roughly $100 \mathrm{~d}$ after exposure, the dissolution rate of ${ }^{238} \mathrm{PuO}_{2}$ may start to increase substantially, leading to rapid decrease of the lung burden and a concurrent increase in urinary excretion of ${ }^{238} \mathrm{Pu}$. (3) Increased bioassay sampling may be appropriate during this period. (4) Depending on the level of urinary ${ }^{238} \mathrm{Pu}$, chelation therapy might be initiated after about $100 \mathrm{~d}$ and continued for several hundred days to reduce uptake and retention of ${ }^{238} \mathrm{Pu}$ in the liver and skeleton. (5) Translocation of ${ }^{238} \mathrm{Pu}$ from the lungs and tracheobronchial lymph nodes may alter interpretation of data from in vivo chest counting.

The cases of human exposure to $\mathrm{UAl}_{\mathrm{x}}$ and ${ }^{238} \mathrm{PuO}_{2}$ and the study of inhaled ${ }^{238} \mathrm{PuO}_{2}$ in dogs illustrate the potential error in the HRTM default assumption of a monotonically declining rate of absorption of material from the respiratory tract. The study on dogs also illustrates the value of animal studies in illuminating the processes controlling the biokinetics of a radionuclide in the body. Such information is required for development of process models that reflect interactions of the physiochemical forms of the radionuclides on biokinetics. Taking this information into account is an important step toward reducing potential errors associated with predictions of the biokinetics of a radionuclide in the body. 


\section{GASTROINTESTINAL TRACT MODEL: UNDERLYING DATA AND INITIAL ASSESSMENTS OF VARIABILITY AND UNCERTAINTY}

This section addresses data and methods used by the ICRP to model the biokinetics of radionuclides in the gastrointestinal tract and resulting doses to tissues of the tract. The gastrointestinal model used by the ICRP since the late 1970s is described briefly. A new "alimentary tract model" (i.e., including all segments of the alimentary tract) to be used in future ICRP documents is described in greater detail. The basis for the new alimentary tract model is discussed, uncertainties in model components and predictions are examined, and variability in rates of transfer of material through different segments of the tract is assessed on the basis of published measurements.

\subsection{The ICRP's gastrointestinal tract model used to derive current dose coefficients}

The gastrointestinal (GI) transit model used in the derivation of current ICRP dose coefficients (Figure 4.1) was adopted by the ICRP in Publication 30 (ICRP, 1979). That model was developed to calculate doses from occupational exposure. It divides the GI tract into four segments or compartments: stomach (St), small intestine (SI), upper large intestine (ULI), and lower large intestine (LLI), and depicts first-order transfer of material from one segment to the next. Material is assumed to transfer from St to SI at the fractional rate of $24 \mathrm{~d}^{-1}$, from SI to ULI at $6 \mathrm{~d}^{-1}$, from ULI to LLI at $1.8 \mathrm{~d}^{-1}$, and from LLI to Feces at $1 \mathrm{~d}^{-1}$. With a few exceptions, absorption is assumed to occur only from SI.

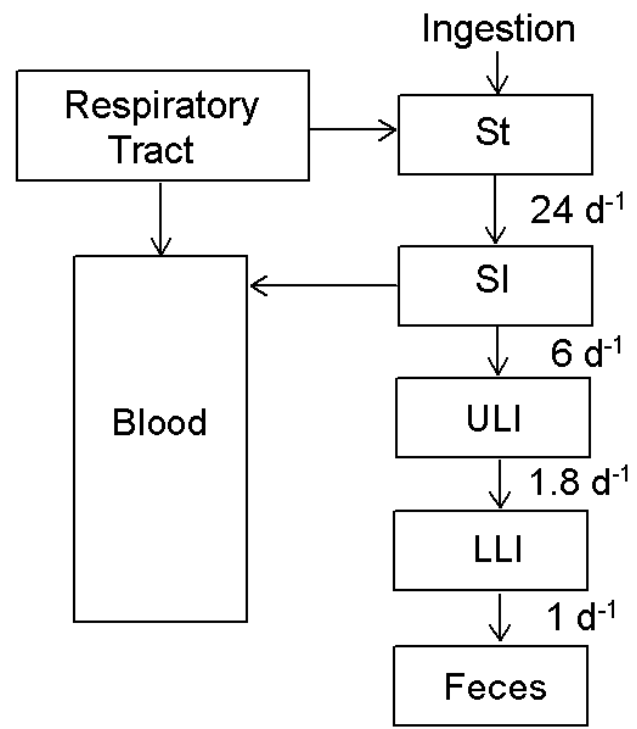

Figure 4.1. Structure of the gastrointestinal tract model used by the ICRP since the late 1970s (ICRP, 1979) 


\subsection{The ICRP's updated GI tract model}

An updated model of the GI tract, called the Human Alimentary Tract Model (HATM), has just been completed by an ICRP task group and adopted by the ICRP. The structure of the HATM is shown in Figure 4.2. The HATM involves greater detail and physiological realism than the ICRP's longstanding GI model but is less complicated than it may at first appear. The organs and fluids represented in Figure 4.2 by dashed boxes are not part of the HATM but are included in the schematic to show connections between the GI model and the respiratory tract model or systemic biokinetic models. Also, many of the compartments and directions of movement of activity were included to address special situations and would not be used in standard applications.

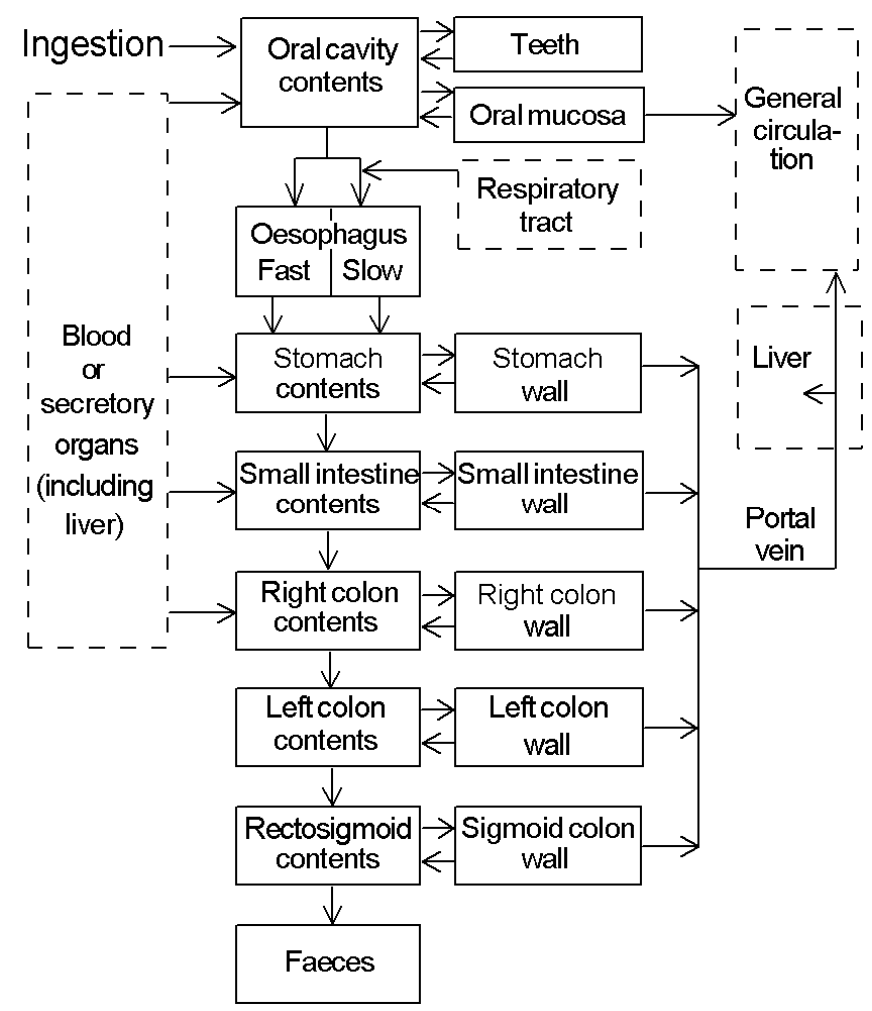

Figure 4.2. Structure of the HATM (dashed boxes included to show connections between the HATM and the respiratory model or systemic biokinetic models) 
In its entirety, the HATM depicts the following processes:

1. entry of a radionuclide into the oral cavity by ingestion or into the esophagus after mechanical clearance from the respiratory tract;

2. sequential transfer through the contents of the oral cavity, esophagus, stomach, small intestine, and segments of the colon, followed by emptying in feces;

3. radionuclide deposition and retention on or between teeth and return to the oral cavity; deposition and retention in oral mucosa or stomach and intestinal walls;

4. transfer from the oral mucosa or walls of the stomach and intestines back into the lumenal contents or into blood (absorption);

5. transfer from various secretory organs or blood into the contents of certain segments of the gastrointestinal tract (secretion).

The HATM divides the colon into the right colon, left colon, and rectosigmoid. The right colon is defined as the cecum, ascending colon, and proximal half of the transverse colon; the left colon is the distal half of the transverse colon plus the descending colon; and the rectosigmoid is the sigmoid colon plus the rectum. This division is often used for diagnostic and experimental examinations of colonic transit, and considerable information is available on transit times through each of these three segments.

First-order kinetics is assumed. This is a considerable simplification of transfer kinetics through the GI tract that obviously does not match the actual discontinuous pattern of fecal excretion of swallowed activity. As discussed later, however, this simplification of the actual kinetics of material in the GI tract does not appear to be a source of major errors in dose estimates for the segments of the tract considered in the HATM.

For computational purposes, each parameter value of the model is represented by a transfer coefficient, also called a rate coefficient or rate constant. A transfer coefficient describes the rate of outflow of a substance from a compartment and is defined as the instantaneous fraction of the contained substance leaving the compartment per unit time.

Application of the model to specific radionuclides requires both generic parameter values and element-specific values. The generic parameter values are those describing bulk flow of material through the lumen of the gastrointestinal tract and are provided in the HATM report. Element-specific parameter values are those describing retention in tissues of the gastrointestinal tract, the rate and location(s) of absorption to blood, and secretion from systemic organs or blood into the lumen of tract. Recommended baseline parameter values for individual elements will be provided in ICRP documents in which the HATM is applied. In some applications it may be feasible to replace generic or element-specific parameter values to reflect case-specific information.

An element-specific transfer coefficient is assumed to be zero if information is not available to support and select a non-zero transfer. As a minimum, information generally is available to derive non-zero transfer coefficients describing total absorption to blood. In the common situation in which absorption is assumed to occur only in the small intestine and there is assumed to be no retention in the wall of the small intestine, the parameters can be calculated from an assumed absorption fraction in the same way as in 
current ICRP documents. The HATM report gives examples of elements for which there is known to be absorption in segments of the tract other than the small intestine or retention or secretion in some segments of the tract.

The HATM report provides baseline regional transit times (Table 4.1). These are central estimates, usually mean values, derived from collected age- and gender-specific data for a given type of material (e.g., solids, liquids, caloric liquids, or non-caloric liquids).

Transit of material through each of the major segments of the tract shows considerable inter- and intra-subject variability even under normal conditions. Large deviations from the norm may result from such factors as unusual diet, constipation, diarrhea, pharmaceuticals, physical activity, stress, and a variety of diseases that affect the nervous system or increase energy requirements. This means that the default transit times given in the HATM document may not be appropriate for specific applications, such as interpretation of fecal monitoring results following accidental exposure.

\begin{tabular}{|c|c|c|c|c|c|}
\hline \multirow{2}{*}{ Segment and material } & \multicolumn{5}{|c|}{ Age group } \\
\hline & Newborn & $1 y$ & $5-15$ y & $\begin{array}{l}\text { Adult } \\
\text { males }\end{array}$ & $\begin{array}{c}\text { Adult } \\
\text { females }\end{array}$ \\
\hline $\begin{array}{l}\text { Mouth } \\
\text { Solids } \\
\text { Liquids } \\
\text { Total diet }\end{array}$ & $\begin{array}{l}-- \\
2 \mathrm{~s}\end{array}$ & $\begin{array}{l}15 \mathrm{~s} \\
2 \mathrm{~s} \\
12 \mathrm{~s}\end{array}$ & $\begin{array}{l}15 s \\
2 s \\
12 s\end{array}$ & $\begin{array}{l}15 s \\
2 s \\
12 s\end{array}$ & $\begin{array}{l}15 \mathrm{~s} \\
2 \mathrm{~s} \\
12 \mathrm{~s}\end{array}$ \\
\hline $\begin{array}{l}\text { Esophagus-fast (90\%) } \\
\text { Solids } \\
\text { Liquids } \\
\text { Total diet }\end{array}$ & $\begin{array}{l}-- \\
4 \mathrm{~s} \\
4 \mathrm{~s}\end{array}$ & $\begin{array}{l}8 \mathrm{~s} \\
5 \mathrm{~s} \\
7 \mathrm{~s}\end{array}$ & $\begin{array}{l}8 \mathrm{~s} \\
5 \mathrm{~s} \\
7 \mathrm{~s}\end{array}$ & $\begin{array}{l}8 \mathrm{~s} \\
5 \mathrm{~s} \\
7 \mathrm{~s}\end{array}$ & $\begin{array}{l}8 \mathrm{~s} \\
5 \mathrm{~s} \\
7 \mathrm{~s}\end{array}$ \\
\hline $\begin{array}{l}\text { Esophagus-residual (10 } \\
\text { Solids } \\
\text { Liquids } \\
\text { Total diet }\end{array}$ & $30 \mathrm{~s}$ & $\begin{array}{l}45 \mathrm{~s} \\
30 \mathrm{~s} \\
40 \mathrm{~s}\end{array}$ & $\begin{array}{l}45 s \\
30 s \\
40 s\end{array}$ & $\begin{array}{l}45 \mathrm{~s} \\
30 \mathrm{~s} \\
40 \mathrm{~s}\end{array}$ & $\begin{array}{l}45 \mathrm{~s} \\
30 \mathrm{~s} \\
40 \mathrm{~s}\end{array}$ \\
\hline $\begin{array}{l}\text { Stomach } \\
\text { Solids } \\
\text { Liquids: caloric } \\
\text { Liquids: non-caloric } \\
\text { Total diet }\end{array}$ & $\begin{array}{l}-- \\
75 \mathrm{~m} \\
10 \mathrm{~m} \\
75 \mathrm{~m}\end{array}$ & $\begin{array}{l}75 \mathrm{~m} \\
45 \mathrm{~m} \\
30 \mathrm{~m} \\
70 \mathrm{~m}\end{array}$ & $\begin{array}{l}75 \mathrm{~m} \\
45 \mathrm{~m} \\
30 \mathrm{~m} \\
70 \mathrm{~m}\end{array}$ & $\begin{array}{l}75 \mathrm{~m} \\
45 \mathrm{~m} \\
30 \mathrm{~m} \\
70 \mathrm{~m}\end{array}$ & $\begin{array}{l}105 \mathrm{~m} \\
60 \mathrm{~m} \\
30 \mathrm{~m} \\
95 \mathrm{~m}\end{array}$ \\
\hline Small intestine ${ }^{a}$ & $4 \mathrm{~h}$ & $4 \mathrm{~h}$ & $4 \mathrm{~h}$ & $4 \mathrm{~h}$ & $4 \mathrm{~h}$ \\
\hline Right colon ${ }^{a}$ & $8 \mathrm{~h}$ & $10 \mathrm{~h}$ & $11 \mathrm{~h}$ & $12 \mathrm{~h}$ & $16 \mathrm{~h}$ \\
\hline Left colon $^{a}$ & $8 \mathrm{~h}$ & $10 \mathrm{~h}$ & $11 \mathrm{~h}$ & $12 \mathrm{~h}$ & $16 \mathrm{~h}$ \\
\hline Rectosigmoid $^{a}$ & $12 \mathrm{~h}$ & $12 \mathrm{~h}$ & $12 \mathrm{~h}$ & $12 \mathrm{~h}$ & $16 \mathrm{~h}$ \\
\hline
\end{tabular}




\subsection{Summary of the database on transit times}

\subsubsection{Sources of information}

The following summary of information on the rate of transit of material through the gastrointestinal tract is a condensation of a chapter and an annex prepared by one of the authors (Leggett) for the HATM report. The extensive bibliography of sources of information used to determine reference values is available from the authors.

\subsubsection{Oral cavity}

The oral cavity was included in the HATM for consideration of special cases, such as ingestion of radionuclides that tend to deposit on the teeth or between the teeth and gums, or ingestion of ultra-short-lived radionuclides. Typical transit times of radionuclides in the mouth are of little importance for most applications of the model.

The length of time that different foods are held in the mouth has been measured in many human subjects, but the data are difficult to interpret in terms of a mean transit time. The time between intake and swallowing of a liquid usually is about 1-3 s, but liquids with a pleasing or interesting taste are sometimes held longer. The time that food is held in the mouth varies from a few seconds to 30 seconds or more, depending on age, the composition and texture of the food, the level of hunger, personal habits, customs, and other factors (Figure 4.3).

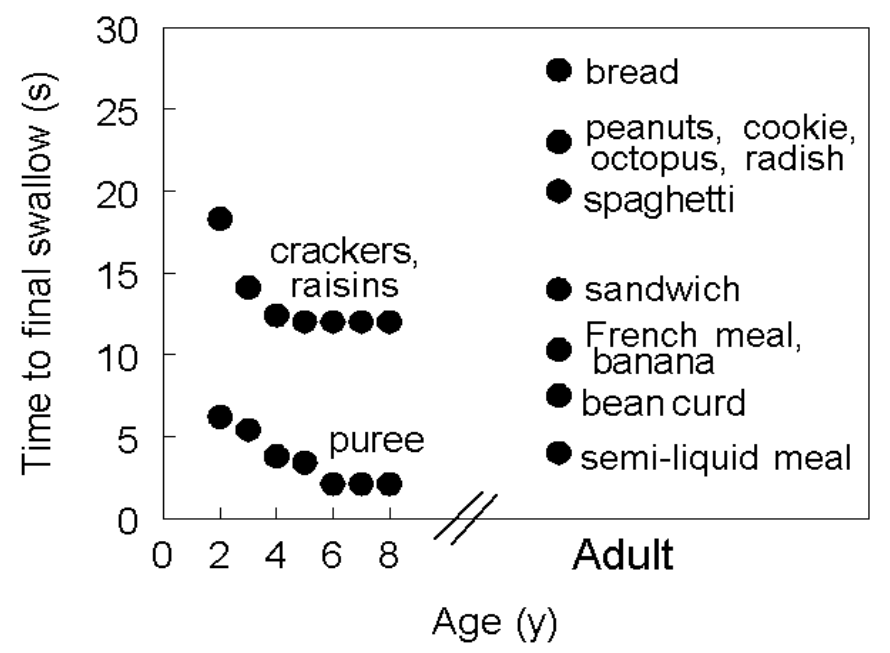

Figure 4.3. Effects of age and food type on residence times in the mouth 


\subsubsection{Esophagus}

When material is swallowed, a coordinated and sequential set of peristaltic contractions produces a zone of pressure that moves down the esophagus with the bolus in front of it. As the bolus approaches the lower esophageal sphincter, the sphincter relaxes, allowing the bolus to enter the stomach. The rate of movement of a bolus varies with its composition and location within the esophagus and the position of the body. Velocities ranging from about $40 \mathrm{~cm} / \mathrm{sec}$ to about $1 \mathrm{~cm} / \mathrm{sec}$ have been reported for different segments of the esophagus and different conditions. Because many people suffer from abnormalities in esophageal transit of swallowed food related either to peristaltic contractions or the esophageal sphincter, the rate of movement of different materials through the esophagus has been investigated in numerous studies involving unhealthy persons and control subjects.

In the HATM, esophageal transit is assumed to have two components, one representing relatively fast transfer of most of the swallowed amount and one representing relatively slow transfer of a small fraction of residual material. Mucus and associated material escalated from the respiratory tract enter the esophagus via the oropharynx. It is assumed that the slow esophageal transit times for the second component of ingested material apply to the transit of all material escalated from the respiratory tract.

For normal subjects, transit times for liquids are generally in the range 3-12 s for the fast component. Esophageal transit of solids varies considerably with the composition of the ingested material and the amount of fluid ingested with the solid. Transit is particularly slow for relatively dry, non-viscous solids. The portion of swallowed material remaining after the initial peristaltic wave and the time that the residual material remains in the esophagus are both highly variable. Measurements involving liquids or semi-solids swallowed by supine or sitting subjects suggest that, on average, residual material may represent about $8-10 \%$ of the swallowed amount. Residual material usually is cleared completely within 30-45 sec and often within a much shorter time, but capsules or solid food ingested without water may remain in the esophagus for several minutes or even hours. Transit times are increased by cold fluids and decreased by warm fluids. Esophageal transit is faster in the upright than the supine position, particularly for solids. Changes with age in esophageal transit in healthy persons appear to be modest.

Esophageal transit can be seriously affected by a hiatal hernia, esophageal reflux, esophageal spasm, and esophageal diverticulosis. Transit is particularly slow in persons with achalasia, a condition in which the lower esophageal sphincter fails to relax during the swallowing mechanism. In achalasia, the residual component often represents half or more of the swallowed material. When achalasia becomes severe, the esophagus may not empty swallowed food for many hours. 


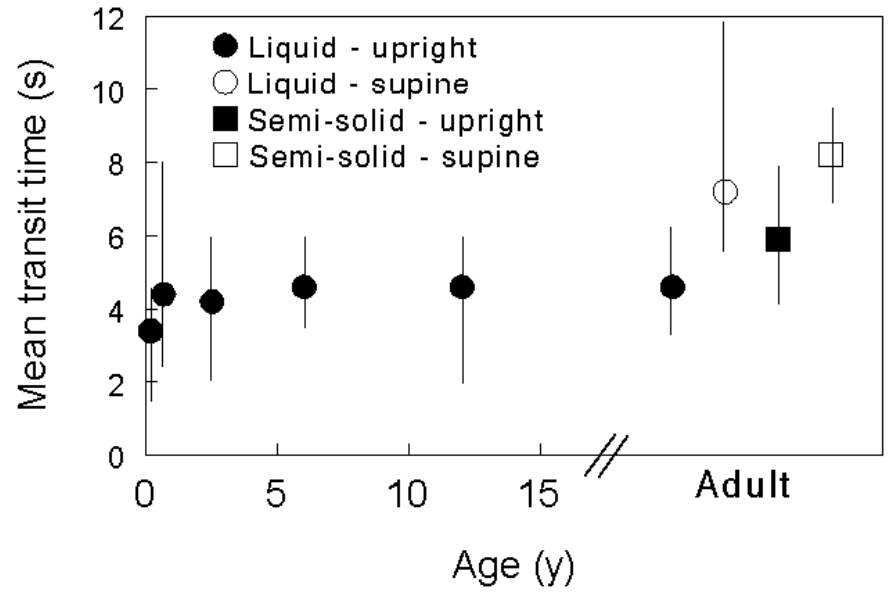

Figure 4.4. Differences with age, food type, and body position in transit times through the lumen of the esophagus, excluding a "slow component" representing a few percent of swallowed material that may reside in the esophagus for periods from about $30 \mathrm{~s}$ to a few hours (vertical lines represent the range of observed values for the fast component)

\subsubsection{Stomach}

The kinetics of gastric emptying is affected by a variety of factors, including composition of the ingested material, gender, and age. Typically, liquids are emptied from the stomach faster than digestible solids, and digestible solids are emptied faster than nondigestible solids. Gastric emptying of liquids usually begins within 1-3 min of their arrival in the stomach and can be described reasonably well by a mono-exponential function, although a lag-phase of several minutes has been reported for liquids of high caloric density. Removal of the solid component typically consists of an initial lag-phase of several minutes in which there is relatively slow emptying, followed by an extended phase of nearly linear emptying. Nondigestible solids are retained in the stomach until the digestible solids have been evacuated. During the latter stages of gastric emptying, a series of migrating motor complexes occurring at regular intervals move distally through the stomach, effectively sweeping the nondigestible solids into the small intestine.

Normally, food is largely removed from the stomach in 2-3 $\mathrm{h}$ and completely removed in $5 \mathrm{~h}$, but the emptying time can be affected by alcohol consumption or other factors.

The rate of emptying of material from the stomach is often reported as a half-emptying time, which refers to the time required for $50 \%$ of the ingested material to be removed from the stomach. Reported central estimates of half-emptying times for solids, all liquids, and non-caloric liquids are summarized in Figure 4.5. The data points indicated as "All liquids" represent primarily measurements of caloric liquids but also include collected data for non-caloric and unspecified liquids. 


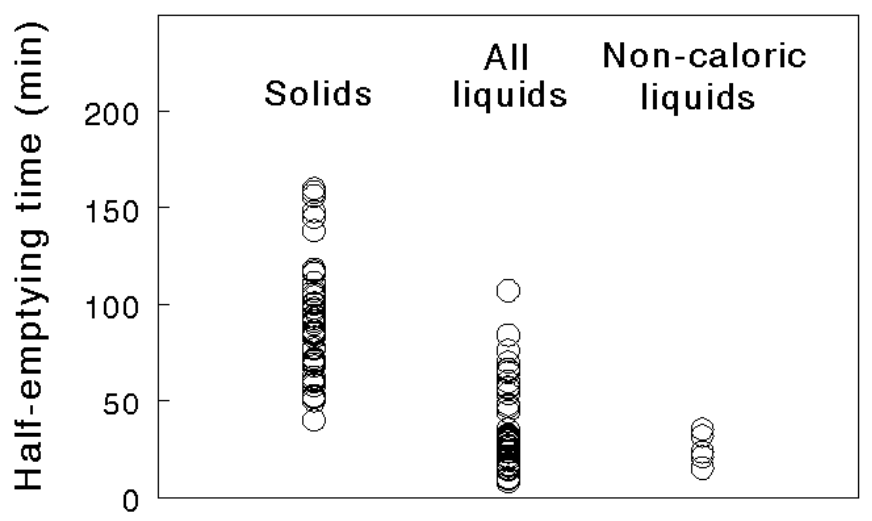

Figure 4.5. Comparison of reported gastric half-emptying times for solids, a variety of test liquids, and non-caloric liquids in healthy adults (circles represent reported central values, usually means, for different study groups)

For healthy adult subjects, reported central values for observed gastric half-emptying times range from 40 to 160 min for solids and from 8 to 107 min for liquids. The means and standard deviations of the collected central values are $91 \pm 30$ min for solids and $36 \pm$ 22 min for liquids.

Reported gastric half-emptying times for meals in infants vary with the type of milk ingested, the maturity of the infant, and the measurement technique. Reported values range from 15 to $100 \mathrm{~min}$ and average about 50-55 min. Estimated half-emptying times of water in healthy infants average about 6 min. Reported gastric emptying times for toddlers, young children, and adolescents generally are within the range of values determined for adults. Gastric emptying appears to be slower in elderly persons than in young and middle-aged adults, but this has not been firmly established.

The rate of gastric emptying of ingested material depends strongly on its composition and the level of nutrients in the jejunum. The emptying time increases nearly linearly with the caloric content of the meal and also is increased substantially by fat.

The emptying time of either solids or caloric liquids is substantially greater on average in women than in men (Figure 4.6). Based on various measures of central tendency including the median, mean, and weighted mean (i.e., weighted by the number of subjects in a study), a typical or central half-emptying time for solids is about 75-80 min in adult males and 100-110 min in adult females. For caloric liquids, a typical half-emptying time is 30-35 min in males and 40-45 min in females. For non-caloric liquids, a typical halfemptying time for either gender may be about 20-25 min. Differences with gender in 
gastric emptying appear to diminish with aging due to an increase in the emptying rate in females, perhaps beginning after menopause.

Men Women

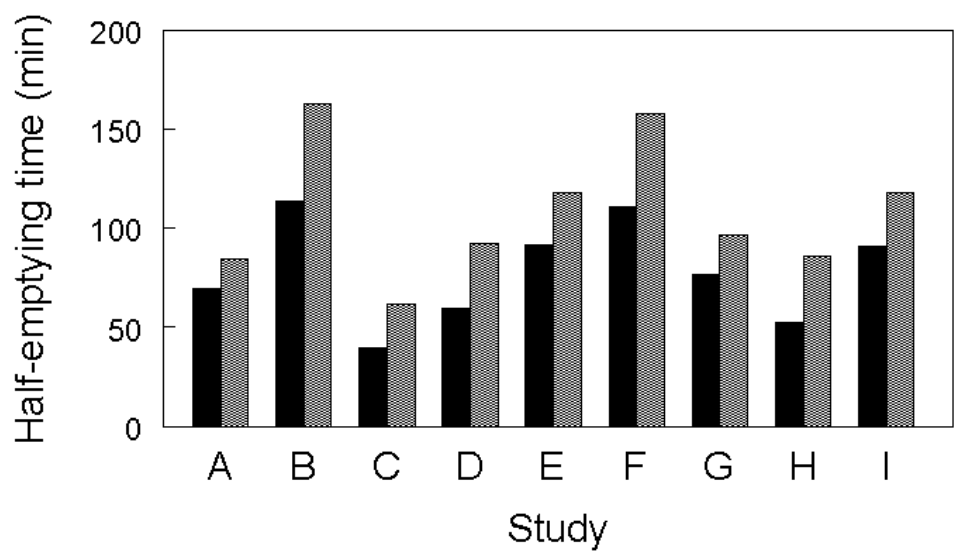

Figure 4.6. Comparison of gastric half-emptying times of solids in adult male and female subjects in nine studies

Gastric emptying times are altered by a number of diseases, including several diseases that affect the nervous system or alter energy requirements. Substantially altered rates are seen in cirrhosis and hypothyroidism. Emptying rates are not consistently increased or decreased in diabetics but appear to be considerably more variable in diabetics than in control subjects. The gastric emptying rate is altered by some pharmaceuticals.

\subsubsection{Small intestine}

Movement of material through the small intestine appears to be a nearly linear process. Measured transfer rates through the small intestine generally are reported as transit times.

The ileum, the distal part of the small intestine, acts as a reservoir and transfers boluses of variable sizes into the colon. Intake of a subsequent meal may stimulate transfer into the colon, but this appears to depend on the composition of the material in the ileum. Some studies suggest faster transfer of liquids than solids in the small intestine, while others indicate that liquids and solids transit the small bowel together, which is assumed in the present model. Fat in the small intestine induces faster transit than proteins but delays ileocolonic transit. Ingestion of oleic acid appears to slow the transit of material through the small intestine.

The transit time through the small intestine shows some variation with contents or other factors but appears to be much less variable than transit through the stomach or colon. Most reported values based on currently favored techniques (e.g., excluding estimates 
based on the frequently applied hydrogen breath test, which are regarded by many investigators as unreliable) fall in the relatively narrow range of 3-4 h. The range of reported central estimates is $1.8-8 \mathrm{~h}$ and the mean \pm standard deviation is $3.9 \pm 1.5 \mathrm{~h}$ (Figure 4.7). Limited comparisons of small intestinal transit in adult males and females and in adults and children have not revealed significant differences with gender or age in the rate of transit of material through the small intestine.

The rate of movement of material through the small intestine may be increased by diarrhea and decreased by constipation but appears from limited information to be much less affected by either condition than is the rate movement through the large intestine. The transit time through the small intestine appears to be altered by some pharmaceuticals. Limited data suggest that the transit time through the small intestine may be reduced by stress and physical exercise and increased during pregnancy.

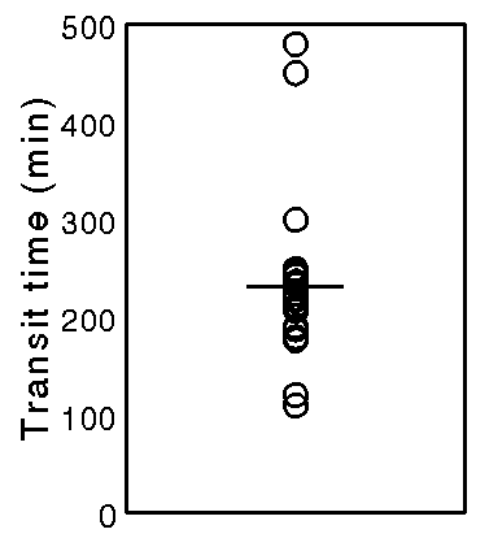

Figure 4.7. Reported small intestinal transit times in subjects without gastrointestinal disorders (circles represent central values determined in separate studies, and the bar represents the mean of these values)

\subsubsection{Colon and rectum}

Flow of material in the colon is slow and highly variable. Periods of contraction between longer periods of quiescence result in mass movements of colonic material a few times during the day. Fluids may move ahead of solids in the proximal right colon, but solids and fluids appear to move together throughout most of the colon.

The rectum serves mainly as a conduit but can also serve as a storage organ. Entry of material from the sigmoid colon usually evokes the recto-anal inhibitory reflex, signaling the need to defecate. The rectum serves as a storage organ when the amount of material received from the sigmoid colon is too small to evoke this reflex or when this reflex is 
neglected. The average residence time of material in the rectum cannot be estimated with much confidence.

Reported central transit times through the total colon vary by about a factor of 4 (range, $17-68 \mathrm{~h}$ ) but are typically in the range $24-48 \mathrm{~h}$. On average, transit times are $30-40 \%$ greater in adult females than in adult males and appear to be greater in adult males than in children. Figure 4.8 summarizes reported mean transit times through the total colon in men, women, and children.

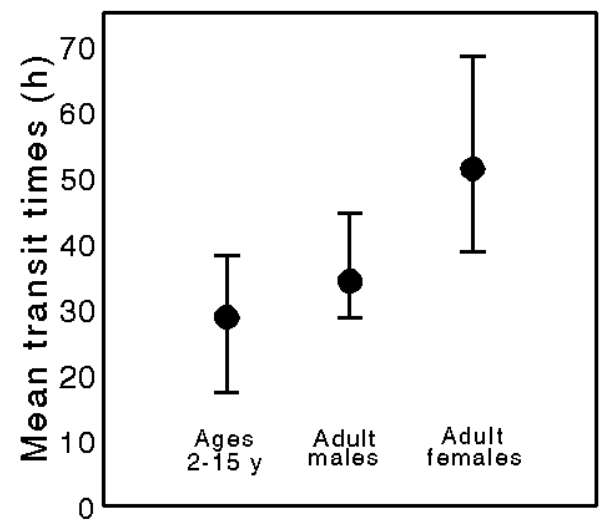

Figure 4.8. Ranges (vertical bars) and overall means (circles) of reported colonic transit times for normal children, adult males, and adult females

Reported central values for transit times in different portions of the colon are summarized in Figure 4.9. Measurements of segmental transit times in relatively large study groups indicate upper $95 \%$ values of roughly $24 \mathrm{~h}$ for the right colon, $36 \mathrm{~h}$ for the left colon, and $40 \mathrm{~h}$ for the rectosigmoid. Average oro-rectal transit times, expected to represent primarily colonic transit, vary substantially from one region of the world to another, presumably due mainly to differences in dietary fiber. For example, measured oro-rectal transit times were longer on average for populations whose diets consisted largely of refined, low-fiber foods (means, 48-83 h) than for populations that ate mainly unrefined, high-fiber foods (means, 34-36 h) or mixed diets (means, 41-47 h).

Colonic transit times may be decreased by a factor of 3 or more in persons with diarrhea and increased by a factor of 3 or more in constipated persons. A number of disease states affect colonic transit. Patients with progressive systemic sclerosis showed an increase in the estimated median gastric emptying time and colonic transit time compared with control subjects but no abnormality in the small intestine transit time. Diarrhea and decreased gastrointestinal transit times are common manifestations of hyperthyroidism, whereas constipation frequently occurs in hypothyroidism. The transit time through the colon may be altered substantially by drugs. 


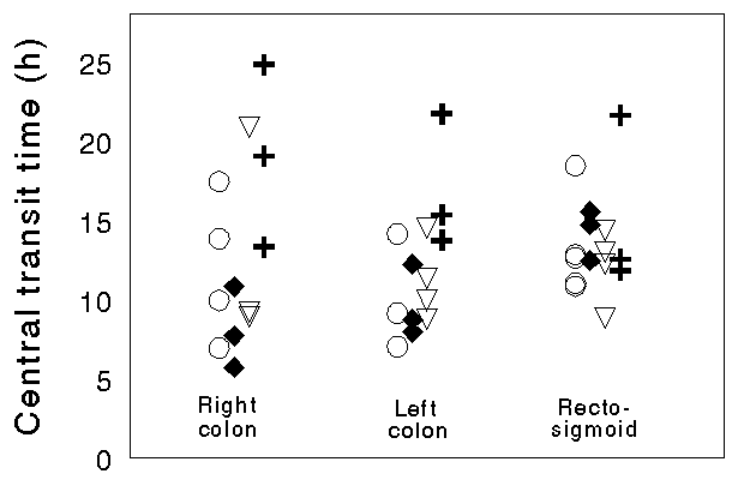

Figure 4.9. Summary of reported transit times in right colon, left colon, and rectosigmoid in normal human subjects (diamonds, children; triangles, adult males; plus signs, adult females; circles, data for adults not separated by gender or of unspecified gender)

\subsection{Contents of segments of the tract and fecal excretion rates}

Development of the HATM was coordinated with the revision (ICRP Publication 89, 2002b) of the ICRP's Reference Man (ICRP Publication 23, 1975). The ICRP's Task Group on Reference Man reviewed data on anatomical and physiological features of the alimentary tract and selected reference values for those features, which were carried over to the HATM. These features included the contents of the stomach and different segments of the intestines, and the mass of feces excreted per day. Reference values for these features are listed in Table 4.2.

Table 4.2. Reference values for contents of segments of the alimentary tract and daily fecal excretion (g) (ICRP Publication 89, 2002b)

\begin{tabular}{cccccccc}
\hline Age & Total & Stomach & $\begin{array}{c}\text { Small } \\
\text { intestine }\end{array}$ & $\begin{array}{c}\text { Right } \\
\text { colon }\end{array}$ & $\begin{array}{c}\text { Left } \\
\text { colon }\end{array}$ & $\begin{array}{c}\text { Recto- } \\
\text { sigmoid }\end{array}$ & $\begin{array}{c}\text { Daily } \\
\text { feces }\end{array}$ \\
\hline Newborn & 144 & 40 & 56 & 24 & 12 & 12 & 24 \\
1 y & 240 & 67 & 93 & 40 & 20 & 20 & 40 \\
5 y & 300 & 83 & 117 & 50 & 25 & 25 & 50 \\
$10 y$ & 420 & 117 & 163 & 70 & 35 & 35 & 70 \\
15 y male & 720 & 200 & 280 & 120 & 60 & 60 & 120 \\
15 y female & 720 & 200 & 280 & 120 & 60 & 60 & 120 \\
Adult male & 900 & 250 & 350 & 150 & 75 & 75 & 150 \\
Adult female & 830 & 230 & 280 & 160 & 80 & 80 & 120 \\
\hline
\end{tabular}




\subsection{Basis for the dosimetric model of the human alimentary tract}

\subsubsection{Limitations of the GI tract model of ICRP Publication 30}

The dosimetric aspects of the HATM represent an important departure from the approach taken by the ICRP in the current GI tract model (ICRP, 1979). In the case of beta radiation, it is assumed in the current model that the dose to the wall of the tract is equivalent to the dose at the surface of the contents. For beta particles of low energy this approach overestimates the dose to the wall and to the cells associated with maintaining the epithelial lining of the wall. For alpha radiations the dose to the wall has been taken as $1 \%$ of the dose at the surface of the contents. This value is not based on calculations of energy deposition but has been applied as a cautious assumption. As judged from limited studies on laboratory animals, it is expected to overestimate dose to sensitive cells in the walls of the gastrointestinal tract.

\subsubsection{A more direct approach taken in the HATM}

With the main exception that the gross morphometry of the GI tract is still represented as a set of idealized geometric figures, an attempt is made in the HATM to address these dosimetric limitations of the GI tract model of ICRP Publication 30. For example, retention of radionuclides in the walls can be specified when information is available (Figure

4.2), and the location of sensitive cells of different regions of the GI tract is modeled explicitly (Table 4.3). The summary of information for the small intestine given below illustrates the dosimetric approach used in the HATM.

\begin{tabular}{lcc}
\hline \multicolumn{3}{l}{ Table 4.3. Target cell depths and masses for regions of the } \\
HATM for adult males & & \\
\hline Region & $\begin{array}{c}\text { Target cell depth } \\
(\mu \mathrm{m})\end{array}$ & $\begin{array}{c}\text { Target cell mass } \\
(\mathrm{g})\end{array}$ \\
\hline Oral cavity & $190-200$ & 0.23 \\
Esophagus & $190-200$ & 0.091 \\
Stomach & $60-100$ & 0.62 \\
Small intestine & $130-150$ & 3.6 \\
Right colon & $280-300$ & 1.3 \\
Left colon & $280-300$ & 1.2 \\
Recto-sigmoid & $280-300$ & 0.73 \\
\hline
\end{tabular}

\subsubsection{Illustration of dosimetric data and assumptions: Small intestine}

The small intestine is used to illustrate the HATM approach for estimating dose to the walls of the intestines from non-penetrating radiations originating in the contents or emitted by radionuclides retained in the walls after uptake from the lumen.

The small intestine is represented as a right circular cylinder. The source within this cylinder is assumed to be uniformly distributed in water that fills the cylinder. Tissue 
comprising the walls of the small intestine is assumed to have a density of $1 \mathrm{~g} \mathrm{~cm}^{-3}$ and the composition of soft tissue defined by the ICRU (1989).

The values for the physiological length of the small intestine are as given in Publication 89 (ICRP, 2002b). Measurements of physiological length are available for newborn infants $(\sim 80 \mathrm{~cm})$ and adult males $(\sim 280 \mathrm{~cm})$, and the central estimates of the length of the small intestine were approximately 1.6 times body height for both ages. For purposes of developing reference values for physiological length of the small intestine, the authors of ICRP Publication 89 assumed the relation holds for ages 1-15 y and for adult females.

The diameter of the lumen of the small intestine changes with age and varies with location within the intestine. Reported data for newborn infants and adults were used to estimate average diameters at each age, assuming that reported values apply to the entire length of the small intestine. For intermediate ages these values were interpolated with average height. Derived values are 1, 1.2, 1.4, 1.6, 2, and $2 \mathrm{~cm}$ for infants and ages 1, 5, 10 , and $15 \mathrm{y}$, and adult, respectively.

The lining of the small intestine possesses gross and microscopic structures for increasing the surface area available for digestive and absorptive processes. The surface is covered with numerous projections, the villi, which serve as absorptive units and which are unique to this segment of the alimentary tract. At their bases are simple tubular invaginations, called crypts of Lieberkühn, that extend to the muscularis mucosae but do not penetrate it. The villi have a single columnar epithelial cover and a core of highly cellular reticular connective tissue. The villi vary in height and form in different regions of the human small intestine. In the HATM, the villus height is taken to be $500 \mu \mathrm{m}$ for age $10 \mathrm{y}$ or greater and $400 \mu \mathrm{m}$ for younger children, based on ultrastructural studies.

The epithelial stem cells responsible for continuous renewal of the epithelial layer are located towards the base of the crypts, immediately above a group of secretory cells, called Paneth cells, at the base of the crypts. Reported measurements of the depth from the intercryptal plate to the top of the Paneth cell zone are $144 \pm 8 \mu \mathrm{m}$ based on data for three adults. The target layer is taken to be 3 cells deep, assumed to be at a depth of $130-$ $150 \mu \mathrm{m}$ from the intercryptal plate. This depth is assumed to apply at all ages, although there is evidence that crypts are somewhat longer in young children.

The geometric model used to estimate energy deposition in sensitive regions of the walls of the intestines from emissions in the lumenal contents is shown in Figure 4.10. Target cells are assumed to form a continuous layer at a defined depth from the lumenal surface. In the case of the small intestine, the target cells in the epithelial lining are assumed to extend from 130 to $150 \mu \mathrm{m}$ below the intercryptal plate. Absorbed fractions of emitted energies for non-penetrating radiations from radionuclides in the lumenal contents are based on the assumption that activity is uniformly distributed throughout the contents of a cylinder. The presence of the villi along the small intestine wall is ignored. This is considered to be a reasonable assumption because gut contents penetrate between the villi as far as the intercryptal plate. It is assumed that gut contents do not penetrate into the 
crypts against the flow of secretions from crypt cells. The presence of mucus on the lumenal surface is ignored.

In considering retention of radionuclides in the wall of the small intestine, the general assumption is made in the HATM that retention is confined to the absorptive region of the villi. This is modeled as a uniform layer of tissue above the intercryptal plate, 500 $\mu \mathrm{m}$ thick in adults and children of 10 years and older and $400 \mu \mathrm{m}$ thick in younger children. Thus, the spaces between villi, occupied by gut contents, are ignored for this purpose. In a limited number of cases, information may be available suggesting retention in deeper mucosal tissues, within the lamina propria between crypts. To model retention in the wall of the small intestine, other than retention in villi, uniform distribution throughout the region of the mucosa beneath the villi is assumed, to a depth of $200 \mu \mathrm{m}$ (base of crypts).

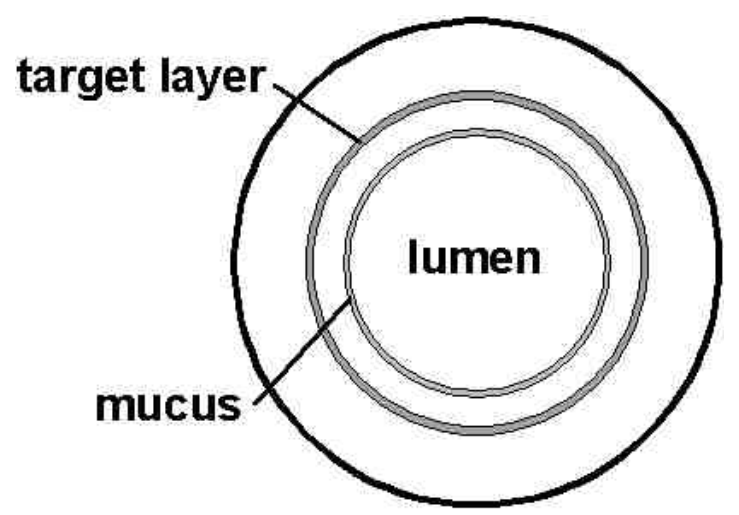

Figure 4.10. Schematic of the geometric model used to estimate energy deposition in radiosensitive cells in the walls of tubular regions of the alimentary tract, from decay of radionuclides in the lumen (presence of mucus on the lumenal surface is ignored; target cells assumed to form a continuous layer at a defined depth from the lumenal surface)

\subsubsection{Systemic activity in the walls of the GI tract}

Most of the ICRP's systemic biokinetic models include compartments with names such as "other tissues" or "other soft tissues" that represent retained activity not accounted for in explicitly identified tissues and fluids. The walls of the HATM regions are included as these non-explicit source regions, taking account of normal processes of circulation and distribution of systemic activity. Thus, the target regions of the HATM can receive doses from this activity as well as from activity in the contents of the lumen or retained in the walls during absorption. For non-penetrating radiation, including electrons and alpha particles, all tissues that constitute "other tissues" are assigned the same dose. This dose estimate is applied to the all target regions of the HATM. Systemic activity often contributes the main component of dose, particularly for compartments of relatively rapid transit, such as the mouth and esophagus, and for radionuclides with tenacious retention in soft tissues. 


\subsubsection{Implications of the HATM for alpha emitters}

When target cells are situated at a depth of greater than around 40 or $50 \mu \mathrm{m}$ in the wall, alpha particles emitted by important radionuclides cannot penetrate the wall to the depth of the target region. Thus, the dose to sensitive cells of the GI tract wall from alpha emissions in the contents is now estimated to be zero due to the depth of the target cells in relation to the range of an alpha particle in tissue.

In the simple geometric model of the stomach, some energetic alpha particles could penetrate to the target region $(60-100 \mu \mathrm{m})$. However, there is a layer of mucus on the lumenal surface of the stomach that is roughly $20 \mu \mathrm{m}$ thick, so that it seems reasonable to assume that alpha particles emitted in the stomach do not reach the sensitive cells.

\subsection{Expert judgments of potential errors in HATM features and predictions}

\subsubsection{The group of experts}

The HATM report discusses sources of error in the model as assessed by the ICRP task group that developed the model. The method of assessment by the task group is essentially expert judgment, considering the underlying information and, where applicable, the sensitivity of predictions to alternate plausible approaches or parameter values. The conclusions of the task group regarding some of the major sources of potential error in the HATM and the sensitivity of model predictions to those potential errors are summarized below.

\subsubsection{Potential errors associated with limitations in model structure}

Two main sources of potential error in the HATM associated with model structure are discussed in the HATM document: the division of the alimentary tract into compartments, and the use of first-order kinetics to describe transfer of material.

Except for the division of the colon, the compartments used to describe transfer of material through the lumen of the alimentary tract represent anatomically and functionally distinct regions of the tract. The colon has been divided in a number of different ways in different radiation protection models. The model of ICRP Publication 30 (1979) divides the colon into the upper large intestine (ULI) and the lower large intestine (LLI), where the ULI includes the ascending and transverse colons and the LLI includes the descending colon, sigmoid colon, and rectum. A model developed for use in nuclear medicine (NCRP, 1998) divides the colon into the ascending colon, transverse colon, and descending colon plus rectosigmoid. The HATM divides the colon into the right colon, left colon, and rectosigmoid, a division often used for diagnostic and experimental examinations of colonic transit. The HATM Task Group concluded that use of this division would allow best estimates of the time-dependent distribution of ingested, inhaled, or secreted activity in the colon, based on modern data. 
The HATM Task Group considered whether the rectum should be represented as a separate compartment, since it is commonly assumed to function mainly as conduit rather than a storage organ. Information found in the literature indicated, however, that the rectum can serve for extended periods as a storage organ and in some cases could contain a substantial portion of the total activity in the alimentary tract. In view of such findings, and because of the difficulties in determining a meaningful transit time separately for the rectum, the Task Group concluded that the rectum should not be treated as a separate compartment.

Representation of movement of material through the lumen of the alimentary tract as a series of first-order transfers between well-mixed pools is a considerable simplification of real events. For example, studies indicate that material traverses the human small intestine in a more or less linear fashion and is transferred to the ascending colon in multi-bolus form. Flow of material in the rectosigmoid region of the colon is slow, variable, and intermittent, with periods of contraction between longer periods of quiescence resulting in mass movements of material a few times during the day.

The assumption of first-order kinetics is made for computational convenience. The emptying half-time for a segment of the tract is set to reproduce central estimates of reported mean transit times of material through that segment, in the absence of radioactive decay. For relatively short-lived radionuclides, first-order kinetics can overestimate decays in the lower regions of the tract because it implies an immediate appearance of some ingested atoms in all regions of the tract. For example, few if any atoms of an ingested radionuclide with half-life $\leq 20 \mathrm{~min}$ are expected to reach the colon, but the HATM predicts on the basis of first-order kinetics that about 3\% of the total decays in the alimentary tract would occur in the colon.

The HATM or any other first-order gastrointestinal model will not yield meaningful predictions of the fecal excretion rate during the early hours after intake. For the case of ingestion of a radionuclide by an adult male, the HATM predicts that, in the absence of absorption to blood, total fecal excretion is about 3\% of the ingested amount after $12 \mathrm{~h}$. Studies indicate that the time to first appearance of ingested markers in feces of healthy adults is more than $12 \mathrm{~h}$ in most cases.

The HATM predicts that total fecal excretion of an unabsorbable marker would be about $22 \%$ of the ingested amount after $1 \mathrm{~d}, 69 \%$ after $2 \mathrm{~d}, 91 \%$ after $3 \mathrm{~d}, 98 \%$ after $4 \mathrm{~d}$, and $>99.5 \%$ after $5 \mathrm{~d}$. These predictions are broadly consistent with reported data for markers used in experimental studies (e.g., carmen red) and within the range of data from accidental intakes of radionuclides, although it appears that fecal excretion often is more nearly complete after about $3 \mathrm{~d}$ than the model predicts. It can be misleading to check the model against small numbers of individual cases due to substantial inter- and intrasubjects variability in the transit time. In some individual cases, nearly all the swallowed amount appears in feces during the first day. In other cases, a few days may elapse before there is any appreciable fecal excretion of ingested material. 


\subsubsection{Potential errors in transit times}

In the years since the development of the Publication 30 model (ICRP, 1979), numerous investigations of the kinetics of material in the gastrointestinal tract have been conducted by improved, non-invasive techniques such as external viewing of radio-labeled foods, liquids, or indigestible substances. While the uncertainties associated with measurement techniques have been substantially reduced, the difficulties involved in determining true transit times should not be underestimated. For example, the physical characteristics of markers used in modern studies may affect colonic transit times. Also, some methods still in common use, such as the hydrogen breath test used to estimate transit through the small intestine, do not appear to provide representative or reproducible results.

Uncertainties also are inherent in the assumptions and algorithms used to translate measurements into estimates of the mean transit time. For example, measurements of colonic transit frequently are based on counts of ingested radio-opaque markers in the regions of interest. Some investigators have argued that this technique may substantially underestimate actual transit times in many cases because the experimental methods may not closely approximate the underlying assumptions of continuous ingestion of markers and attainment of steady-state by the time of counting. The extent of underestimate may vary considerably from one study to another due to differences in numbers and patterns of administration of the marker and times of measurement.

Considering the limitations of measurement techniques and reported average residence times in different segments of the alimentary tract in healthy individuals, the HATM Task Group made judgments concerning the potential error in the transit time of material in major segments of the tract in the adult male. The transit time of material in the esophagus was judged to be known with a factor of 2 (UF $=2$; see definition of UF in Section 2). The transit time of material in the stomach, small intestine, right colon, left colon, or rectosigmoid colon in the adult male was judged to be known within a factor of about $1.5(\mathrm{UF}=1.5)$.

Based on the UFs for transit times, effective dose coefficients and equivalent dose coefficients to the colon were calculated for ingestion of ${ }^{90} \mathrm{Sr},{ }^{106} \mathrm{Ru}$ and ${ }^{239} \mathrm{Pu}$ by adult males. In the cases of ${ }^{90} \mathrm{Sr}$ and ${ }^{106} \mathrm{Ru}$ the resulting UFs for colon dose were 1.5 and 1.4 respectively, which are nearly the same as that for transit time, reflecting their close association. For ${ }^{239} \mathrm{Pu}$, colon dose arises solely from activity absorbed to blood, and variations in transit time have no effect on colon dose because colon dose arises almost entirely from activity absorbed to blood.

For ${ }^{106} \mathrm{Ru}$ the colon dose from activity in the contents makes an important contribution to effective dose, and the potential errors in transit times yield a UF for effective dose of about 1.2. For ${ }^{90} \mathrm{Sr}$ and ${ }^{239} \mathrm{Pu}$, however, colon dose contributes little to the effective dose, which is virtually unaffected by potential errors in estimated gastrointestinal transit times. 


\subsubsection{Simplifications of anatomical features of the alimentary tract}

The dimensions and geometrical configurations of the structures of the tract were formerly estimated from measurements on cadavers, and those estimates often did not closely reflect conditions in the living body. More accurate determination of the geometry of the gastrointestinal tract of a living person has become possible with the advent of external visualization techniques with high resolution. Nevertheless, it remains difficult to determine typical sizes, shapes, and relative positions of structures of the tract with high accuracy due to the considerable variability in these features from one person to another and from one body position to another in the same person.

The HATM Task Group assigned UFs of about 1.4 to the dimensions (e.g., length, width, or internal diameter of a segment of the intestines) of most structures within the alimentary tract. They noted that UF $=1.4$ for the length of a region would lead to the same UF for dose to the region from activity in the contents if all other parameters remain unchanged but pointed out that the length of a region may be correlated with the transit time. For example, an increase in length of a region could lead to an increase in transit time by the same factor with the result that the dose is unaltered. This would tend to reduce UF for dose to below 1.4.

The HATM Task Group examined the sensitivity of dose from beta emitters in the intestinal contents to the uncertainty in the diameter of the intestines. Strontium-90 $($ mean beta energy $=0.20 \mathrm{MeV})$ and ${ }^{90} \mathrm{Y}$ (mean beta energy $=0.94 \mathrm{MeV}$ ) were chosen to represent relatively low and relatively high beta energies. Based on an assigned UF of 1.4 for the diameter of the intestines, the resulting UF for dose to the wall is about 1.9. In contrast to most parameters, this is a case where UF for the result (dose) is larger than that in the parameter (diameter). This is because the UF in the cross-sectional area of the section, which is an indicator of the extent of energy absorption within the lumen, is 2 for

a UF of 1.4 in the diameter. However, such uncertainties in colon dose from ${ }^{90} \mathrm{Sr} /{ }^{90} \mathrm{Y}$ will have a negligible effect on committed effective dose which is dominated by doses to tissues from activity absorbed to blood.

\subsubsection{Uncertainties in the location of target regions for cancer induction}

Doses may be calculated separately for the mucosal layer of each region of the HATM. For penetrating radiations, the average dose to the walls of each region may be used as a measure of the dose to the mucosal layer. For non-penetrating alpha and beta particle emissions originating in the contents of the tract, the dose is dependent on assumptions regarding the location of target cells for cancer induction. For each region of the alimentary tract, the target is taken to be the stem cells that are located in the basal layer of the stratified squamous epithelia of the mouth and esophagus and within the crypts that penetrate the mucosal layer in the stomach and small and large intestines.

The HATM Task Group considered potential errors in estimates of dose to the colon due to uncertainties in the location of the target cells. They considered both the uncertainty in the depth of the stem cells and the uncertainty as to whether it is only the stem cells that 
should be regarded as targets. Table 4.4 compares colon doses for different assumptions of target location, normalized to the default assumption that the sensitive cells form a continuous layer at a depth of $280-300 \mu \mathrm{m}$ from the lumenal surface of the colon. The possibility addressed in the last column of Table 4.4, that target cells could be distributed over depths from 0-300 $\mu \mathrm{m}$, is an extreme situation based on observations in human subjects of preneoplastic cells on the lumenal surface but not in crypts.

\begin{tabular}{|c|c|c|c|c|}
\hline \multicolumn{5}{|c|}{$\begin{array}{l}\text { Table 4.4. Differences (\%) in dose coefficients for the colon, compared to the } \\
\text { default case }{ }^{\mathrm{a}} \text {, resulting from considerations of target depth in the mucosa, } \\
\text { considering ingestion by adult males }\end{array}$} \\
\hline \multirow[t]{2}{*}{ Nuclide } & \multicolumn{4}{|c|}{ Assumed location of the target region - depth from lumen, $\mu \mathrm{m}$} \\
\hline & $220-240$ & $340-360$ & $200-300$ & $0-300$ \\
\hline $\begin{array}{l}\text { Sr-90 } \\
\text { Ru-106 } \\
\text { U-234 } \\
\text { Pu-239 }\end{array}$ & $\begin{array}{l}\% \\
3 \% \\
0 \% \\
0 \%\end{array}$ & $\begin{array}{l}-6 \% \\
-2 \% \\
0 \% \\
0 \%\end{array}$ & $\begin{array}{l}5 \% \\
2 \% \\
0 \% \\
0 \%\end{array}$ & $\begin{array}{c}21 \% \\
8 \% \\
148 \% \\
317 \%\end{array}$ \\
\hline
\end{tabular}

${ }^{\mathrm{a}}$ Default case assumes a target depth of $280-300 \mu \mathrm{m}$

\subsubsection{Potential errors in element-specific parameter values of the HATM}

The above discussion addresses potential errors associated with generic features of the HATM. Errors in predictions of the HATM also arise from element- or radionuclidespecific parameter values, such as uptake from the contents of the tract to blood or retention in the walls of the tract. The databases for specific radionuclides are addressed in a later section. Due to the importance of gastrointestinal uptake on doses from intake of radionuclides, however, it is worthwhile to summarize the current state of information on absorption of essentially all elements as assessed by the HATM Task Group.

The potential error in fractional uptake from the gastrointestinal tract to blood varies considerably from one element to another. In a relative sense, errors in estimated in fractional uptake are likely to be smallest for elements that are known to be nearly completely absorbed, including hydrogen (as tritium), carbon, sodium, chlorine, potassium, bromine, rubidium, molybdenum, iodine, cesium, thallium, fluorine, sulfur, and germanium. A value UF in the range 1.1-1.5 might be appropriate for each of these elements, depending on the quality and completeness of the data base for individual elements. Average uptake from the gastrointestinal tract is also reasonably well established for several frequently studied elements whose absorption is incomplete but represents at least a few percent of intake, such as copper, zinc, magnesium, technetium, arsenic, calcium, strontium, barium, radium, lead, iron, manganese, cobalt, and uranium. UF for these elements would also vary with the element and generally would be greater than 1.5 but no more than about 3 . Relative errors generally are greater for the remaining elements due to the sparseness of direct observations on human subjects (e.g. ruthenium, silver), inconsistencies in reported absorption fractions (e.g. beryllium, antimony, silicon), or absorption too low to be determined with much precision under most conditions (e.g. most actinide and lanthanide elements). Absorption of a few poorly absorbed elements such as plutonium, americium, and curium has been studied under 
controlled conditions in human subjects, and average uptake in the adult may be known within a factor of $3-4$ for these elements. Relative errors may be greatest for several elements whose absorption has not been studied in man but for which animal data or other indirect evidence indicates absorption of at most a few hundredths of a percent, such as samarium, gadolinium, dysprosium, erbium, thulium, actinium, yttrium, and scandium. Absorption fractions for these elements are order-of-magnitude estimates. 


\section{DEMONSTRATION OF DIFFERENT LEVELS OF KNOWLEDGE OF THE BIOKINETICS AND DOSE PER UNIT INTAKE FOR SPECIFIC RADIONUCLIDES}

This section demonstrates different levels of information available to estimate tissue doses from exposure to different radionuclides, summarizes information on the variability of biokinetics and dose per unit intake or exposure in each case, and critically evaluates previously published uncertainty assessments on those cases. Five radionuclides are addressed in order of decreasing levels of $\mathrm{H} 1$ data (i.e., direct information on their behavior in humans): ${ }^{137} \mathrm{Cs},{ }^{90} \mathrm{Sr},{ }^{60} \mathrm{Co},{ }^{106} \mathrm{Ru}$, and ${ }^{252} \mathrm{Cf}$. Discussions of the two extreme cases, ${ }^{137} \mathrm{Cs}$ and ${ }^{252} \mathrm{Cf}$, illustrate how recent critical reviews of the literature have led to improved biokinetic models and in turn to reduction in the potential errors associated with predictions of models currently used by the ICRP.

\subsection{Cesium-137: Level 1 (Highest level of information)}

\subsubsection{Overview}

Cesium-137 is one of the most extensively studied and best understood radionuclides with regard to biological behavior and tissue doses following intake. Cesium is a chemical and physiological analogue of the essential element potassium. Most commonly encountered forms of cesium are highly soluble and nearly completely absorbed to blood from the gastrointestinal or respiratory tract. Whole-body retention of cesium has been studied in numerous human subjects, some exposed to elevated concentrations of radiocesium in the environment and others exposed for experimental purposes. Wholebody retention can be described reasonably well as a sum of two exponential terms, with the long-term component representing the preponderance of the systemic deposit. Autopsy studies on environmentally exposed humans as well as experimental studies on laboratory animals indicate that cesium is somewhat uniformly distributed in the body, with skeletal muscle gradually becoming the dominant repository. The half-time of the long-term component has been found to vary with age, gender, diet, race, muscle mass, pregnancy, and elevation above sea level (Lloyd et al., 1966, 1973; Zundel et al., 1969; Leggett, 1986; ICRP, 1989; Leggett et al., 2003). Cesium-137 is a beta emitter but is accompanied by the short-lived daughter ${ }^{137 \mathrm{~m}} \mathrm{Ba}$ that emits relatively energetic gamma radiation that allows external measurement of internal deposits.

\subsubsection{Biokinetics}

\subsubsection{Physiological analogues}

Cesium follows the movement of potassium in the body and competes with potassium and its chemical analogue rubidium for both active and passive membrane transport across cell membranes, but cesium is generally transported less readily than potassium or rubidium by these processes. Quantitative differences in transport rates across membranes result in a total-body retention time for cesium about three times greater than 
that for potassium and two times greater than that for rubidium. Cesium, potassium, and rubidium generally have much higher concentrations inside cells than in extracellular fluids, in contrast to the largely extracellular alkali metals, $\mathrm{Na}$ and Li (Eisenman, 1963; Kernan, 1972; Leggett et al., 2003).

Variability in cesium biokinetics in the population can be explained largely by differences in the mass of potassium in the body; the level of the hormone aldosterone in blood, which affects release of potassium and cesium from cells and the urinary excretion rate of cesium; and the level of potassium in diet (Leggett, 1986). The latter two factors are related, e.g., ingestion of a high-potassium diet increases aldosterone secretion.

\subsubsection{Behavior in the respiratory tract}

Information on the behavior of cesium in the respiratory tract was reviewed in ICRP Publication 71 (ICRP, 1995b) and recently by a task group of the ICRP (to be published in an ICRP document). Data on inhalation of cesium as chloride, nitrate, or sulfate, or as residues from a reactor fuel cooling pond indicate relatively rapid and nearly complete absorption from the respiratory tract, consistent with highly soluble material. Results of in vitro studies of the solubility of irradiated fuel fragments containing radiocesium suggest that the material was only moderately soluble. In animal studies of inhalation of cesium incorporated into fused aluminosilicate particles, only a small fraction of material was rapidly absorbed from the lungs, and the rest was absorbed at rates consistent with moderately soluble or relatively insoluble material, depending on the experiment. No data on variability of respiratory kinetics associated with age, gender, or other individual characteristics was found.

\subsubsection{Fractional uptake from the gastrointestinal tract}

Information on gastrointestinal uptake of cesium was reviewed by Harrison et al. (2001). Human volunteer studies using ${ }^{137} \mathrm{Cs}$ in soluble inorganic form (e.g., as ${ }^{137} \mathrm{CsCl}$ ) have shown virtually complete absorption ( 0.99). Cesium incorporated into relatively insoluble particles is less available for absorption. For example, absorption of ${ }^{137} \mathrm{Cs}$ from irradiated reactor fuel particles was less than 0.1 in rats. Estimated absorption after ingestion of real or simulated fallout by human volunteers has varied from less than 0.1 to nearly complete absorption. Measurements of uptake of ${ }^{137} \mathrm{Cs}$ in human volunteers who ingested meat (venison, mutton, caribou) contaminated after the Chernobyl accident have given values in the range $0.6-0.99$. No data on variability of gastrointestinal absorption of cesium associated with age, gender, or other individual characteristics was found.

In ICRP documents, complete absorption of cesium from the gastrointestinal tract is assumed, except after transfer of moderately soluble or insoluble cesium from the respiratory tract to the gastrointestinal tract. For Type M, fractional absorption of 0.2 is assumed for infants and 0.1 for ages 1 y and greater. For Type S, absorption of 0.02 is assumed for infants and 0.01 for ages $1 \mathrm{y}$ and greater. 


\subsubsection{Systemic biokinetics}

\subsection{ICRP model}

The ICRP's current systemic biokinetic model for cesium was originally developed for application to workers (ICRP, 1979). The model does not have a biologically realistic structure. It is essentially a "retention function", i.e., a sum of exponential terms that describe a net rate of movement of absorbed activity from tissues to excretion pathways. The implied model structure is shown in Figure 5.1.

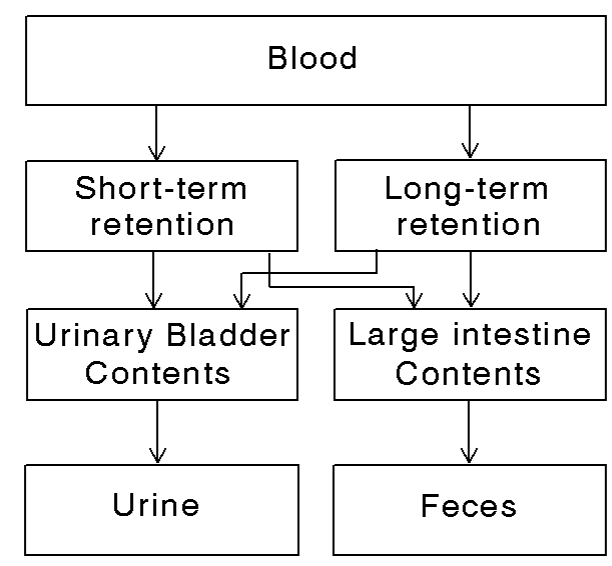

Figure 5.1. Structure of ICRP's current systemic biokinetic model for cesium

Whole-body retention at $t$ days after injection is described by a sum of two exponential terms:

$$
R(t)=a \exp \left(-0.693 t / T_{1}\right)+(1-a) \exp \left(-0.693 t / T_{2}\right) .
$$

Values a $=0.1, \mathrm{~T}_{1}=2 \mathrm{~d}$, and $\mathrm{T}_{2}=110 \mathrm{~d}$ were applied to the worker in Publication 30 . An age-specific version of the cesium model was adopted in ICRP Publication 56 (1989). The parameters a, $T_{1}$, and $T_{2}$ were assumed to vary with age, reflecting more rapid turnover of cesium in children than in adults. The parameter values for ages up to $15 \mathrm{y}$ were taken from a model of Leggett (1986) with the same formulation and with age- and gender-specific parameter values $\mathrm{a}, \mathrm{T}_{1}$, and $\mathrm{T}_{2}$ related to total-body potassium for ages $1 \mathrm{y}$ or greater and based on measured half-times in infants. For the adult, the parameter values used in ICRP Publication $56\left(\mathrm{a}=0.1, \mathrm{~T}_{1}=2 \mathrm{~d}\right.$, and $\left.\mathrm{T}_{2}=110 \mathrm{~d}\right)$ were carried over from the cesium model in ICRP Publication 30 (1979) but do not differ greatly from the parameter values derived for adult males by Leggett (1986). For all age groups, cesium is assumed to be uniformly distributed in the body.

A number of studies of ${ }^{137} \mathrm{Cs}$ retention in human subjects have been published since the development of the current ICRP model, and a detailed physiologically based systemic biokinetic model for Cs reflecting the current database has been developed (Leggett et al., 2003). The model has been tentatively selected by an ICRP task group for use in the revision of ICRP dose guidance for workers and will be referred to as the "ICRP's proposed model". 
The structure of the proposed model is shown in Figure 5.2. The model is constructed around a blood flow model (data type P) and, in contrast to the two-exponential model for cesium currently used by the ICRP, depicts a non-homogeneous distribution of activity the body soon after entry into blood. For derivation of parameter values, information on the biokinetics of cesium in the human body (H1) or laboratory animals (A1) was supplemented with data on potassium and rubidium and information on patterns of discrimination between these three elements by tissues (H2, A2). As illustrated in Figure 5.2 for whole-body retention data in adult males, model predictions are consistent with the database on the time-dependent distribution and retention of cesium in the human body at early, intermediate, and late times after exposure. The model depicts a small component of very long-term retention observed in subjects involved in the accident in Goiania, Brazil, and in some animal studies. This component does not represent an important contribution to dose per unit intake of radiocesium but can be important with regard to interpreting bioassay data collected long after exposure. The model can be used to describe the behavior of cesium during Prussian Blue treatment by reducing or eliminating transfer from the gastrointestinal contents back to blood.

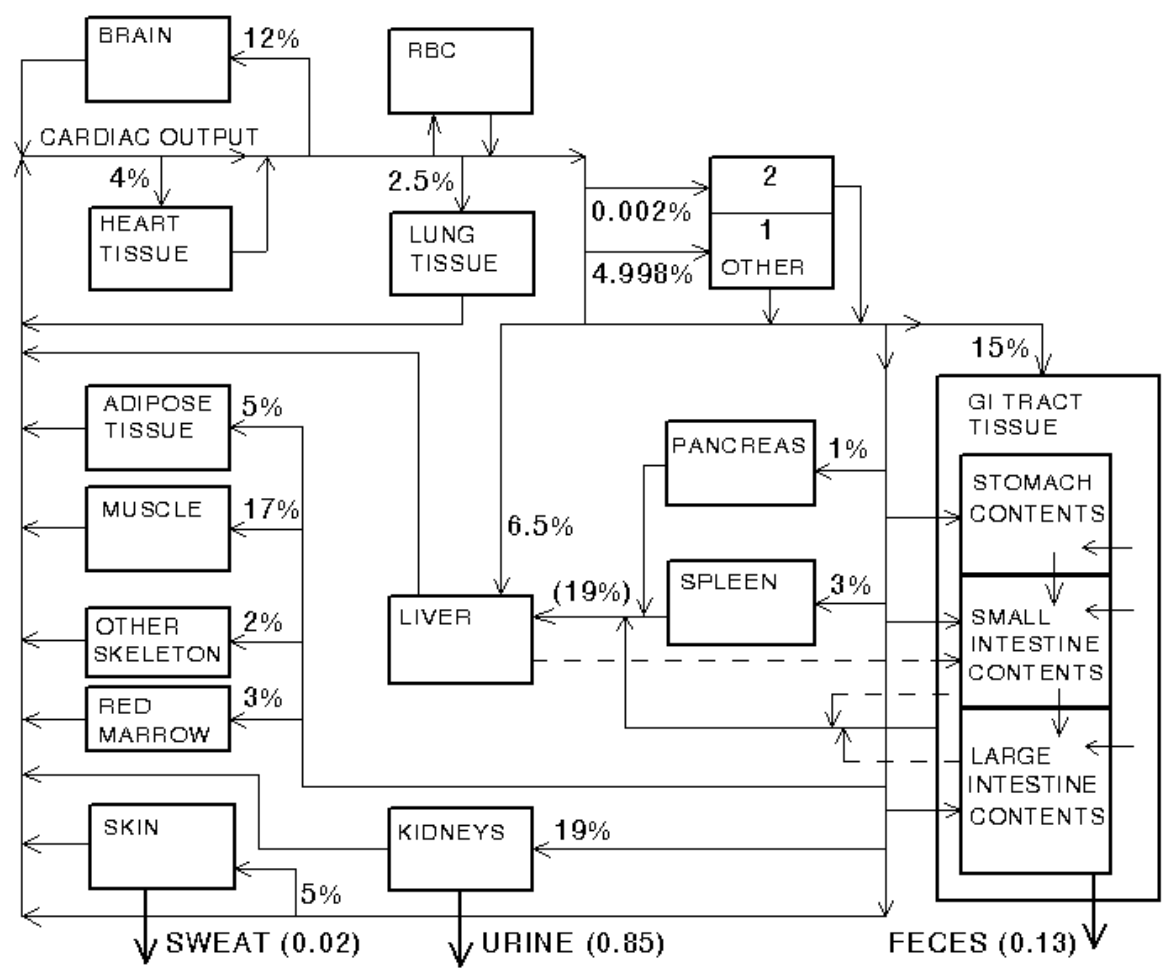

Figure 5.2. Structure of a physiologically based biokinetic model for Cs in the human body (Leggett et al., 2003) (solid arrows represent plasma flow and broken arrows represent flow not involving plasma; percentages indicate distribution of cardiac output; numbers beside SWEAT, URINE, and FECES are fractions of cumulative excretion) 


\subsection{Database for adult male humans}

The biokinetics of cesium in healthy adult males is well understood from experimental studies involving human volunteers, follow-up of subjects receiving occupational or environmental exposure to ${ }^{137} \mathrm{Cs}$, and autopsy measurements of the distribution of ${ }^{137} \mathrm{Cs}$ in the body. Whole-body retention of acutely ingested ${ }^{137} \mathrm{Cs}$ has been followed in a number of adult males until little of the intake remained in the body (Figure 5.3). A number of these studies had been completed by the mid-1970s and the results were considered in the development of the ICRP's current retention-function model for cesium.

Results of 14 studies of the long-term half-time in healthy adult males yield mean longterm half-times in the range 79-133 d with an overall mean of about $97 \mathrm{~d}$ (Figure 5.4). The number of subjects per study varied from 2 to 239 . Inter-subject variability within a given study generally was small, with a typical coefficient of variation of about $20 \%$ and a typical geometric standard deviation of about 1.2 (Lloyd et al., 1973; Leggett, 1986; ICRP, 1989; Leggett et al., 1998, 2003).

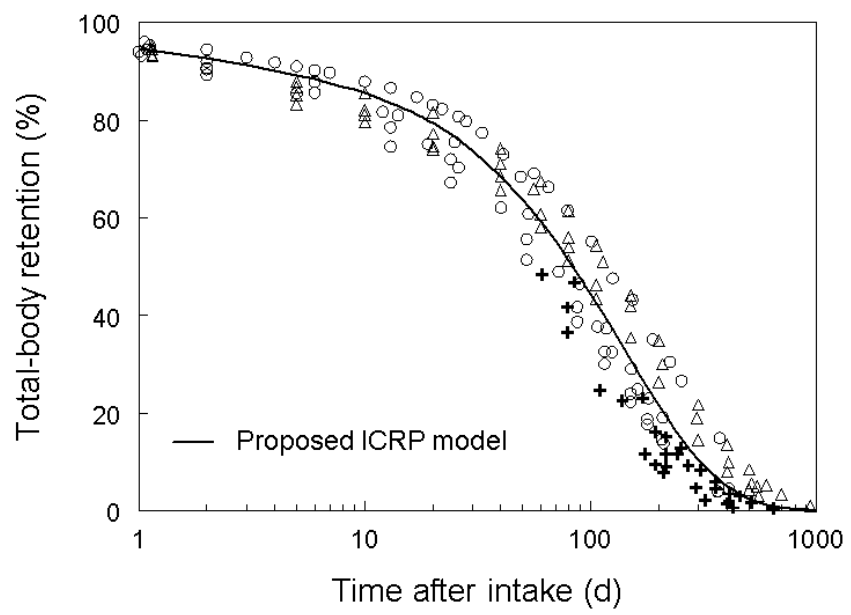

Figure 5.3. Whole-body retention of ingested cesium as predicted by the proposed cesium model update (Leggett et al., 2003) and measured in several adult male humans followed for an extended period

\subsection{Differences with gender}

In at least eight studies, retention half-times have been measured in adult female as well as adult male subjects (Figure 5.5). Although there is some overlap in individual halftimes for male and female subjects, the mean half-time for the female subjects is $15-35 \%$ lower than that for male subjects in each of these studies. The long-term half-time of cesium in the body usually is reduced during pregnancy to about two-thirds of the value when not pregnant, perhaps due to increased aldosterone levels in blood during 
pregnancy (Lloyd et al., 1966; Zundel et al., 1969; Leggett, 1986; ICRP, 1989; Melo et al., 1997; Thornberg and Mattsson, 2000).

Schwartz and Dunning (1982) collected data from the literature on the equivalent biological half-time of ${ }^{137} \mathrm{Cs}$ in the human body. The equivalent half-time is estimated from simultaneous measurement of the total body content and the excretion rate and assumes that total-body ${ }^{137} \mathrm{Cs}$ is a well-mixed pool. They determined means of $96 \pm 23 \mathrm{~d}$ (range 47-152 d) from data 116 adult males and $65 \pm 29 \mathrm{~d}$ (range 30-141 d) for 29 adult females.

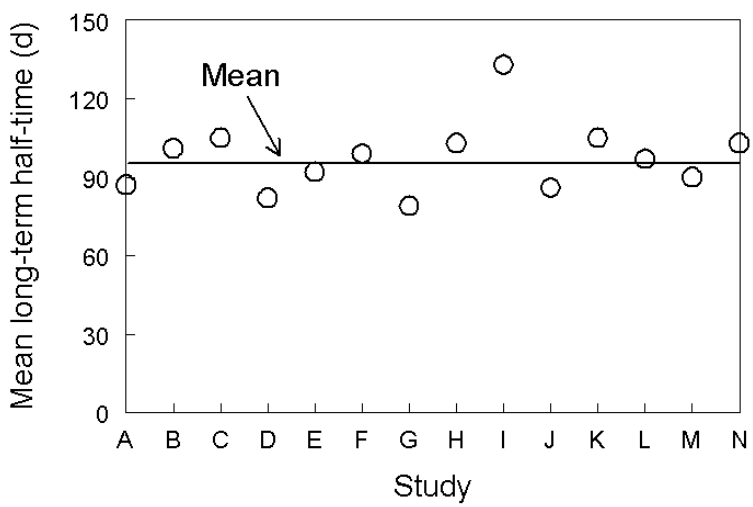

Figure 5.4. Mean long-term half-times (d) for total-body retention of cesium in 14 studies involving healthy adult male subjects. The number of subjects in individual studies ranged from 2 to 239.

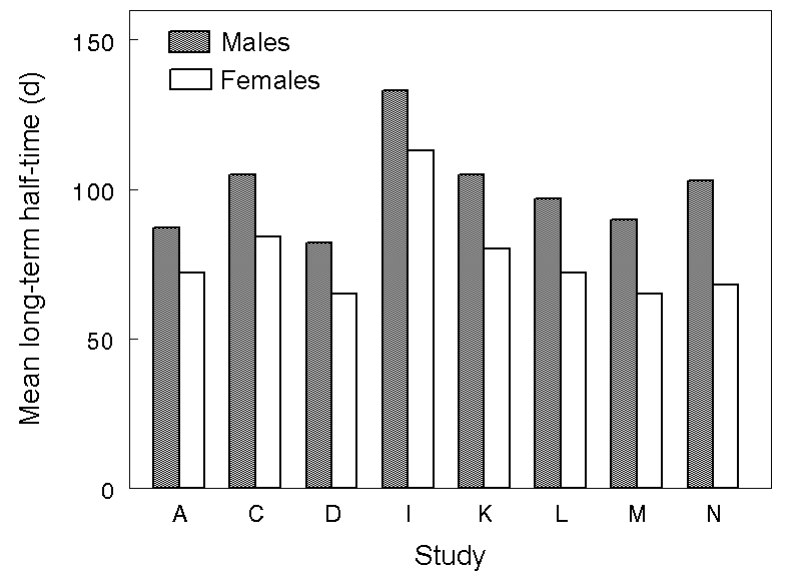

Figure 5.5. Comparative total-body half-times of cesium in adult males and adult females in eight studies 


\subsection{Variation with age}

Variation with age in the retention time of radiocesium in the human body has been investigated in controlled studies, in subjects exposed to contamination from the Chernobyl accident or other sources of environmental contamination, and in subjects exposed in the accident in Goiania, Brazil. Data from three studies (Lloyd et al., 1973; Lebedev and Yakovlev, 1993; Melo et al., 1997) are shown in Figure 5.6. Typical longterm half-times as a function of age and gender are given in Table 5.1 (McCraw, 1965; Lloyd et al., 1973; Leggett, 1986; Lebedev and Yakovlev, 1993; Melo et al., 1997; Leggett et al., 1998).

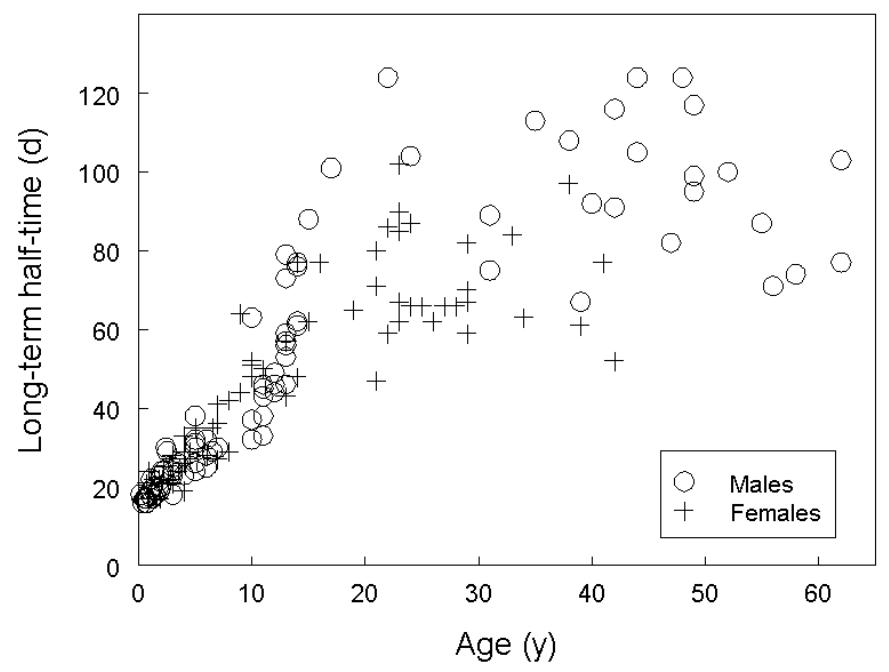

Figure 5.6. Measured ${ }^{137} \mathrm{Cs}$ whole-body retention half-times at different ages

Table 5.1. Typical age- and gender-specific long-term retention half-times for cesium in the human body

\begin{tabular}{ccc}
\hline & \multicolumn{2}{c}{ Long-term half-time (d) } \\
\cline { 2 - 3 } Age & Males & Females \\
\hline Infant (100 d) & 17 & 17 \\
1 y & 19 & 19 \\
$2 y$ & 22 & 22 \\
$5 y$ & 32 & 32 \\
$10 y$ & 46 & 46 \\
$15 y$ & 75 & 65 \\
$35 y$ & 100 & 75 \\
$60 y$ & 85 & 65 \\
\hline
\end{tabular}




\subsection{Effects of disease}

The biokinetics of cesium may be strongly affected by diseases that affect the behavior of potassium or its mass in the total body or in skeletal muscle. For example, persons with Duchenne dystrophy have low concentrations of potassium in skeletal muscle and also show rapid removal of ${ }^{137} \mathrm{Cs}$ from the body due to its limited uptake or retention by skeletal muscle, which is typically the main repository of cesium in the body (Lloyd et al., 1973; Leggett, 1986).

\subsection{Behavior of radioactive progeny}

In the estimation of dose from intake of radionuclides, it must be considered that radioactive progeny produced in the body may have different biokinetics from the parent radionuclide. Approximately $94.6 \%$ of decays of ${ }^{137} \mathrm{Cs}$ go to ${ }^{137 \mathrm{~m}} \mathrm{Ba}$. Despite its short half-life $(2.55 \mathrm{~min}),{ }^{137 \mathrm{~m}} \mathrm{Ba}$ apparently migrates to a large extent from sites of decay of its parent, ${ }^{137} \mathrm{Cs}$, to sites that accumulate barium when introduced directly into blood. For example, at 4-7 days after administration of ${ }^{137} \mathrm{Cs}$ to rats, ${ }^{137 \mathrm{~m}} \mathrm{Ba}$ was found to exceed equilibrium proportions in bone and plasma by factors of 3 and 14, respectively. Liver tissue was slightly deficient in ${ }^{137 \mathrm{~m}} \mathrm{Ba}$, whereas bone marrow and spleen showed little deviation from equilibrium (Wasserman et al., 1959). Sensitivity tests indicate that the standard assumption that ${ }^{137 \mathrm{~m}} \mathrm{Ba}$ decays at its site of production may overestimate doses from intake of ${ }^{137} \mathrm{Cs}$ to most tissues but could underestimate doses to barium repositories (e.g., bone surfaces and colon) by $50 \%$ or more. In the best estimates of ${ }^{137} \mathrm{Cs}$ doses from inhalation or ingestion given in this document, independent biokinetics of ${ }^{137 \mathrm{~m}} \mathrm{Ba}$ is assumed; i.e., ${ }^{137 \mathrm{~m}} \mathrm{Ba}$ produced in the body is assigned the current ICRP model for barium.

\subsubsection{Dosimetry}

The decay schemes for ${ }^{137} \mathrm{Cs}$ and its daughter ${ }^{137 \mathrm{~m}} \mathrm{Ba}$, including half-lives, modes of decay, and energy spectra, have been studied extensively. Cesium-137 is a beta emitter that decays $94.6 \%$ of the time to ${ }^{137 \mathrm{~m}} \mathrm{Ba}$, which emits penetrating gamma radiations. Per nuclear transformation of ${ }^{137} \mathrm{Cs}$, the energy of emitted gamma and $\mathrm{x}$ rays for the chain of decays is $0.664 \mathrm{MeV}$ and the average energy of emitted beta radiation is $0.249 \mathrm{MeV}$ (ICRP, 1983). The beta radiation generally will be absorbed in the organ in which it originates. Exceptions occur when source and target tissues are separated by only a few microns (e.g., when the source is trabecular bone and the target is trabecular marrow), in which case the dosimetry becomes an important source of uncertainty in estimates of dose to the target region. For emitted gamma radiations, energy absorbed fractions can be estimated with reasonable accuracy for different combinations of source and target organs, but some error arises from incomplete knowledge of shapes and relative geometries of organs in the living body. 


\subsubsection{Variability of dose per unit intake or per unit exposure}

As discussed earlier, the biokinetics of cesium varies with age, gender, muscle mass, diet, pregnancy, and other factors. Age has a particularly large effect on biokinetics, with retention time in infants typically less than one-fifth the retention time in adults. There are environmental factors that may affect the biokinetics of cesium such as ambient temperature or height above sea level, but these effects generally appear to be modest.

With regard to dose per unit intake, the effects of age, gender, and muscle mass on the retention time of cesium are largely offset by corresponding changes in body size, with infants being the only subgroup of the population with noticeably higher estimated dose per unit intake than the young adult male. This is illustrated in Table 5.2 for the case of ingestion of ${ }^{137} \mathrm{Cs}$. Note that this table addresses relatively large subpopulations rather than individuals, but none of the available data suggested that individuals within these subgroups might receive substantially higher doses than the average member of the subpopulation.

When differences in intake of ${ }^{137} \mathrm{Cs}$ in food or air are considered, the young adult male is estimated to receive higher doses from ingestion of ${ }^{137} \mathrm{Cs}$ than other members of the population (Table 5.2). The estimates in Table 5.2 are based on current information as interpreted by the authors of this report rather than the ICRP's current systemic model, but differences from ICRP model estimates are modest.

\begin{tabular}{|c|c|c|c|}
\hline \multirow[t]{2}{*}{ Group } & \multirow{2}{*}{$\begin{array}{l}\text { Dose per } \\
\text { unit activity } \\
\text { ingested }\end{array}$} & \multicolumn{2}{|c|}{$\begin{array}{c}\text { Dose per unit activity } \\
\text { in food supply }\end{array}$} \\
\hline & & $\begin{array}{l}\text { Based on } \\
\text { calorie intake }\end{array}$ & $\begin{array}{c}\text { Based on } \\
\text { potassium intake }\end{array}$ \\
\hline Infants & 1.6 & 0.3 & 0.7 \\
\hline 1 -y-old & 0.9 & 0.2 & 0.5 \\
\hline 5 -y-old & 0.8 & 0.4 & 0.5 \\
\hline 10 -y-old & 0.8 & 0.5 & 0.6 \\
\hline 15 -y-old male & 0.9 & 0.7 & 0.8 \\
\hline 15 -y-old female & 0.9 & 0.6 & 0.6 \\
\hline 25 -y-old male & 1.0 & 1.0 & 1.0 \\
\hline 25 -y-old female & 0.9 & 0.6 & 0.6 \\
\hline 65-y-old male & 0.8 & 0.6 & 0.8 \\
\hline 65-y-old female & 0.7 & 0.4 & 0.5 \\
\hline
\end{tabular}

\subsubsection{Existing uncertainty assessments for intake of ${ }^{137} \mathrm{Cs}$}

Uncertainty assessments have been performed for different aspects of the biokinetics or dosimetry of ${ }^{137} \mathrm{Cs}$, including fractional absorption from the gastrointestinal tract (Harrison et al., 2001), cumulative activity in the total body after acute uptake of a unit activity to blood (Leggett et al., 1998), and dose per unit of ingested or inhaled activity 
(Schwarz and Dunning, 1982; Bogen et al., 1997; NCRP, 1998; NRC-CEC, 1998; Apostoaei and Miller, 2004). Methods of assessment have included expert judgment based on a consensus of several national or international experts (e.g., Harrison et al., 2001; NCRP, 1998), collection of independent judgments of several international experts (e.g., NRC-CEC, 1998); and parameter uncertainty analyses by small groups of investigators (e.g., Schwarz and Dunning, 1982; Apostoaei and Miller, 2004). The general conclusion is that ${ }^{137} \mathrm{Cs}$ is well understood with regard both to its biological behavior and the dose per unit intake following intake of highly soluble forms of this radionuclide. Methods and conclusions of a typical parameter uncertainty analysis are described below.

Apostoaei and Miller used parameter uncertainty analysis to assess uncertainty in dose coefficients for ingestion of ${ }^{137} \mathrm{Cs}$. Their analysis was based on the age- and genderspecific retention-function model of Leggett (1986):

$\mathrm{R}(\mathrm{t})=\mathrm{a} \exp \left(-0.693 \mathrm{t} / \mathrm{T}_{1}\right)+(1-\mathrm{a})\left(-0.693 \mathrm{t} / \mathrm{T}_{2}\right)$.

As discussed earlier, the ICRP adopted this model in ICRP Publication 56 (1989) for ages $1-15$ y but kept the gender-independent model of ICRP Publication 30 to adults. In the model, each of the coefficients and the short- and long-term half-times $\mathrm{T}_{1}$ and $\mathrm{T}_{2}$ (days) is linearly related to the amount of potassium $\mathrm{K}_{\mathrm{tb}}$ in the total body (Leggett, 1986).

Apostoaei and Miller assigned an uncertainty distribution for $\mathrm{K}_{\mathrm{tb}}$ by propagating the variability in reported concentration of potassium and total-body mass. Based on reported values for measured absorption of ${ }^{137} \mathrm{Cs}$ in inorganic form or in food, the uncertainty in GI uptake of ${ }^{137} \mathrm{Cs}$ was assigned a triangular distribution having a minimum of $80 \%$ and a mode and maximum of $100 \%$. They assumed that ${ }^{137} \mathrm{Cs}$ is uniformly distributed in the body, the sites of decay of ${ }^{137 \mathrm{~m}} \mathrm{Ba}$ produced in vivo are the same as that of the parent ${ }^{137} \mathrm{Cs}$, and energy from beta radiation is completely absorbed in the organ in which the decay occurs. They noted that the fraction of energy from gamma emissions from ${ }^{137 \mathrm{~m}} \mathrm{Ba}$ absorbed by an organ is uncertain due to such factors as "the lack of knowledge about the precise position and shape of each target organ" and "statistical uncertainties introduced by the methods used to solve the radiation transport problem". "In the absence of better information, the uncertainty in the absorbed fraction for gamma radiation for all target organs was assumed to be 50\%" and a "log-uniform distribution" was assigned. Their uncertainty distributions for the mass of the total body or specific organs were estimates of inter-individual variability based on information from ICRP's Reference Man document (1975).

On the basis of their parameter uncertainty analysis, Apostoaei and Miller concluded that the probability distributions for ingestion dose coefficients for ${ }^{137} \mathrm{Cs}$ can be fitted reasonably well by either normal or lognormal distributions. The derived GSD was 1.24 for males and 1.40 for females, with the larger uncertainty for females due to the larger variation of the amount of potassium in the body. The magnitude of the uncertainty was judged to be similar among organs "because the main contributor to the uncertainty in the dose coefficients...is the biokinetic model, which affects all organs in the same way." The GI uptake fraction, mass of each organ, and absorbed fraction (gamma) for individual organ did not appear to contribute greatly to the total uncertainty. The 
estimated 95\% confidence intervals encompassed the ICRP's dose coefficients for adults, but the means and medians of the derived uncertainty distributions for specific organs were lower than the ICRP values by $2-18 \%$ for males and $2-27 \%$ for females. The main reason for these differences from ICRP was that the ICRP's long-term half-time of $110 \mathrm{~d}$ for adults, carried over from ICRP Publication 30 to Publication 89, is longer than those of the Leggett model for average adult males $(\sim 100 \mathrm{~d})$ and average adult females $(\sim 85 \mathrm{~d})$.

Apostoaei and Miller explained that their results reflect inter-individual variability as well as lack of knowledge about model parameters

"Dose coefficients ... are regarded as central estimates for large populations... Many physical and physiological parameters influencing absorption and movement of a radioactive substance in the human body vary from one individual to another, introducing uncertainties due to inter-individual variability. In addition, some parameters are not well known, and one must therefore deal with uncertainty due to lack of knowledge about the true means and variances of these parameters. The two types of uncertainty can be combined if the assessment end point is the lack of knowledge in the dose to a reference individual, but they must be separated if the assessment end point is variability of doses in a population..."

For example, Apostoaei and Miller used observed variability in total body potassium or organ masses as uncertainty distributions for these parameters, and they assigned an arbitrary uncertainty distribution to the absorbed fraction for gamma radiation mainly due to lack of knowledge. Thus, their results apparently represent statements concerning uncertainty in the dose to an individual from ingestion of ${ }^{137} \mathrm{Cs}$, which would indicate that their results overstate uncertainty as defined in the present report.

An issue related to the definition of uncertainty is that the level of confidence that should be placed in a dose coefficient may depend strongly on the nature of its application. The uncertainty distributions derived by Apostoaei and Miller for ingested ${ }^{137} \mathrm{Cs}$ depend on their relatively narrow uncertainty distribution for fractional uptake of ${ }^{137} \mathrm{Cs}$ from the GI tract. That distribution may be appropriate, for example, for ingestion of ${ }^{137} \mathrm{Cs}$ biologically incorporated in food or ingestion of soluble inorganic forms of ${ }^{137} \mathrm{Cs}$, but it would not be appropriate for application to ingestion of particulate ${ }^{137} \mathrm{Cs}$ deposited on raw fruit or vegetables following a reactor accident or nuclear bomb test, because uncertainty in gastrointestinal uptake of ${ }^{137} \mathrm{Cs}$ would be relatively large in this case.

An issue mentioned by Apostoaei and Miller but not considered in their analysis is model structure. The model that they applied has two retention components, and it is assumed that activity associated with each component is uniformly distributed in the body. This is generally considered to be a reasonable assumption because ${ }^{137} \mathrm{Cs}$ is fairly uniformly distributed compared with most radionuclides and because the short-lived daughter

${ }^{137 m} \mathrm{Ba}$ emits penetrating radiations that would help to correct for any error in the assumption of a uniform distribution. However, calculations based on the detailed cesium model shown in Figure 5.2 indicate that doses to some tissues may be overestimated by as much as $40 \%$ due to the assumption of a uniform distribution. The main reason is that ${ }^{137} \mathrm{Cs}$ accumulates largely in skeletal muscle, and beta emissions from 
decay of ${ }^{137} \mathrm{Cs}$ in skeletal muscle will not reach more sensitive tissues such as lung and colon.

Apostoaei and Miller discussed the possibility of migration of the short-lived daughter

${ }^{137 \mathrm{~m}} \mathrm{Ba}$ from the site of decay of ${ }^{137} \mathrm{Cs}$ but did not include this uncertainty in their analysis. In fact, it is not feasible to address this source of error within the model structure that they used, because a substantially different structure is required to model the biokinetics of barium. Barium- $137 \mathrm{~m}$ represents more than half of the energy involved in the decays of this chain, and experimental evidence indicates that it can migrate from ${ }^{137} \mathrm{Cs}$ to sites where absorbed Ba would normally be found in high concentration including the skeleton and the colon. Our computations indicate that the effective dose from ingestion of ${ }^{137} \mathrm{Cs}$ might be underestimated by as much as $30-40 \%$ due to the assumption that ${ }^{137 \mathrm{~m}} \mathrm{Ba}$ remains with ${ }^{137} \mathrm{Cs}$ in the body. While the problem of migration of progeny from the parent is not major for ${ }^{137} \mathrm{Cs}$ dosimetry, it is a major issue for some other important radionuclides.

\subsection{Strontium-90: Level 2}

\subsubsection{Overview}

Strontium-90 is used to illustrate a second level of information on biological behavior and dose per unit intake, that is, relatively good information but not as detailed or easily interpreted as that for ${ }^{137} \mathrm{Cs}$. As is the case for ${ }^{137} \mathrm{Cs}$, there is a large biokinetic database for ${ }^{90} \mathrm{Sr}$, including much $\mathrm{H} 1$ data. Also like ${ }^{137} \mathrm{Cs},{ }^{90} \mathrm{Sr}$ has a close chemical and physiological analogue (calcium) that has been studied extensively as an essential element. Nevertheless, dose per unit intake cannot be determined as narrowly for ${ }^{90} \mathrm{Sr}$ as for ${ }^{137} \mathrm{Cs}$ due to the less complete and more variable absorption of ${ }^{90} \mathrm{Sr}$ to blood, the more heterogeneous distribution of ${ }^{90} \mathrm{Sr}$ in the body, and the lack of penetrating radiations from the ${ }^{90} \mathrm{Sr}$ chain, which prevents measurement of ${ }^{90} \mathrm{Sr}$ or ${ }^{90} \mathrm{Y}$ in vivo and presents some dosimetric difficulties for some source and target regions that are narrowly separated such as in the lungs and skeleton.

\subsubsection{Biokinetics}

\subsubsection{Physiological analogues}

Strontium is an alkaline earth element that follows the biological behavior of its close chemical analogue calcium, but the biokinetics of calcium and strontium differ somewhat due to discrimination between these elements by biological membranes. Experimental data indicate that strontium is less effectively absorbed from the intestines, more effectively excreted by the kidney, and less readily removed from bone surfaces than calcium (see reviews in Leggett, 1992; ICRP, 1993). However, strontium is a better quantitative tracer for calcium than are the heavier alkaline earth elements, barium and radium. Calcium and strontium are excreted primarily in urine, while barium and radium are excreted mainly in feces, with the total excretion rate soon after injection being higher 
for the heavier alkaline earths than for calcium and strontium (Harrison et al. 1967). All four elements have similar skeletal uptake and distribution at early times after injection (Ellsasser et al., 1969; Wood et al., 1970; Liniecki, 1971; Stather, 1974; Lloyd et al., 1976). Within a few months after administration of isotopes of calcium, strontium, barium, or radium, nearly all of the remaining total-body activity is associated with bone mineral (ICRP, 1973; Schlenker, 1982).

\subsubsection{Behavior in the respiratory tract}

Information on the behavior of different forms of inhaled strontium in the respiratory tract was reviewed in ICRP Publication 71 (1995) and recently by an ICRP task group (to be published in an ICRP document). Data are available for workers accidentally exposed to various strontium compounds (H1) and for laboratory animals exposed to well characterized forms of strontium (A1). Data for accidental intake of strontium as chloride or carbonate indicate that most of the deposited activity is cleared to blood at a rate reasonably consistent with the ICRP's HRTM parameter values for Type F material (fast dissolution and absorption). Rapid clearance of strontium inhaled as chloride or sulfate also has been observed in laboratory animals. Measurements following the accidental inhalation of mixtures of fresh fission products indicate Type $\mathrm{M}$ behavior of most of the strontium present. Results of an in vitro study on airborne fission products are consistent with assignment to Type F. Strontium inhaled in fused aluminosilicate particles or polystyrene have shown low absorption to blood, consistent with Type S. Studies of the rate of clearance of inhaled strontium titanate (SrTiO3) from the lungs in laboratory animals and an accidentally exposed subject indicate slow absorption consistent with HRTM parameter values for Type S material (slow dissolution and low absorption to blood) or perhaps Type M material (moderate dissolution and absorption) in some cases.

\subsubsection{Fractional uptake from the gastrointestinal tract}

Information on gastrointestinal uptake of strontium was reviewed by Harrison et al. (2001). Data from several controlled studies on human subjects ingesting inorganic forms of radiostrontium in solution or known quantities of radiostrontium incorporated in food indicate that absorption is generally in the range $8-35 \%$ and averages about $20-25 \%$. Absorption of strontium from fallout was in the range $0-9 \%$ in 10 subjects and averaged about 3\%. For simulated fallout prepared as glass microspheres, average absorption was about $16 \%$ (range $6-25 \%$ ). A number of factors have been found to increase absorption, including fasting and low dietary levels of calcium, $\mathrm{Mg}$ and P. Milk diets and vitamin D may also increase absorption. Overnight fasting or a low calcium diet can increase absorption by a factor of 2 or more. Results from animal studies generally support the quantitative estimates based on data for human subjects. Animal data show substantially elevated absorption of strontium early in life. Data on uptake and retention of

environmental ${ }^{90} \mathrm{Sr}$ by humans suggest but do not firmly established elevated absorption during periods of rapid bone growth, including adolescence. Absorption of strontium administered as titanate $\left(\mathrm{SrTiO}_{3}\right)$ to rats show low levels of absorption, on the order of $1 \%$. 


\subsubsection{Systemic biokinetics}

\subsection{ICRP model}

The ICRP's systemic model for strontium was developed within a generic model structure (Figure 5.7) for a class of elements that follow the movement of calcium in the skeleton. This class includes the alkaline earth elements barium and radium and more distant physiological relatives of calcium such as lead and uranium. The structure and parameter values for the alkaline earth elements were developed in the early 1990s (Leggett, 1992) and was first used by the ICRP in its series on age-specific doses to members of the public (ICRP 1993, 1995). The following paragraphs explain the basis for the generic model structure for calcium-like elements and selection of the ICRP's parameter values for strontium.

Plasma is treated in the generic model structure as a uniformly mixed pool, although this is not strictly true for calcium and its close physiological analogues. For example, slightly more than half of plasma calcium is in ionic form and readily exchangeable with extravascular calcium, while the rest is bound to proteins and not "diffusible" (Borle 1981). Since the alkaline earth elements are known to have little affinity for red blood cells (RBC), the RBC compartment is not considered explicitly in applications to these elements, but it used in the models for lead and uranium.

Kinetic analysis of plasma disappearance curves for normal subjects intravenously injected with calcium or strontium tracers indicates that these elements initially leave plasma at a rate of several hundred plasma volumes $\mathrm{d}^{-1}$ and equilibrate rapidly with an extravascular compartment roughly three times the size of the plasma pool (Heaney 1964, Harrison et al. 1967, Hart and Spencer 1976). This extravascular compartment presumably consists largely of interstitial fluids. At times greater than a few minutes after injection, a transfer rate from plasma of about $15 \mathrm{~d}^{-1}$ yields a reasonable fit to plasma disappearance curves for strontium or calcium tracers. The models for strontium and calcium do not depict the extremely rapid removal of activity during the early minutes but assign a removal rate from plasma of $15 \mathrm{~d}^{-1}$.

The behavior of strontium in soft tissues was modeled by relating strontium-specific data on uptake and retention in soft tissues to the kinetics of calcium, for which more information was available ( $\mathrm{H} 2$ data). Kinetic analysis of data for human subjects administered radiocalcium indicate that readily exchangeable calcium in soft tissues represents an estimated $0.35 \%$ of total-body calcium in a middle-aged adult human (Heaney 1964, Borle 1981, Schlenker et al. 1982). Plasma contains about $0.03 \%$ of totalbody calcium in the adult. The rapid-turnover soft-tissue compartment ST0 shown in Figure 5.7 was used to represent the roughly threefold larger extracellular pool in exchange with plasma calcium. Thus, ST0 contains about $0.09 \%$ of total-body calcium. This leaves $0.26 \%$ for the intermediate-term compartment, ST1. If ST1 receives a fraction $\mathrm{F}$ of calcium leaving plasma and loses calcium to plasma with a half-time of $\mathrm{T} \mathrm{d}$, then $\mathrm{F}$ and $\mathrm{T}$ are related by the equation $0.0003 \times \mathrm{F} \mathrm{x} 15 \mathrm{~d}^{-1}=0.0026 \times(\ln (2) / \mathrm{T}) \mathrm{d}^{-1}$. In this equation, 0.0003 is the fraction of total-body calcium in plasma and $15 \mathrm{~d}^{-1}$ is the rate 
of removal of calcium from plasma. Thus, the product of $\mathrm{F}$ and $\mathrm{T}$ should be about 0.4 . A good fit to data on retention of ${ }^{45} \mathrm{Ca}$ in human soft tissues over the first $124 \mathrm{~d}$ after injection (Schulert et al., 1959) is obtained with $\mathrm{F}=0.1$ and $\mathrm{T}=4 \mathrm{~d}$.

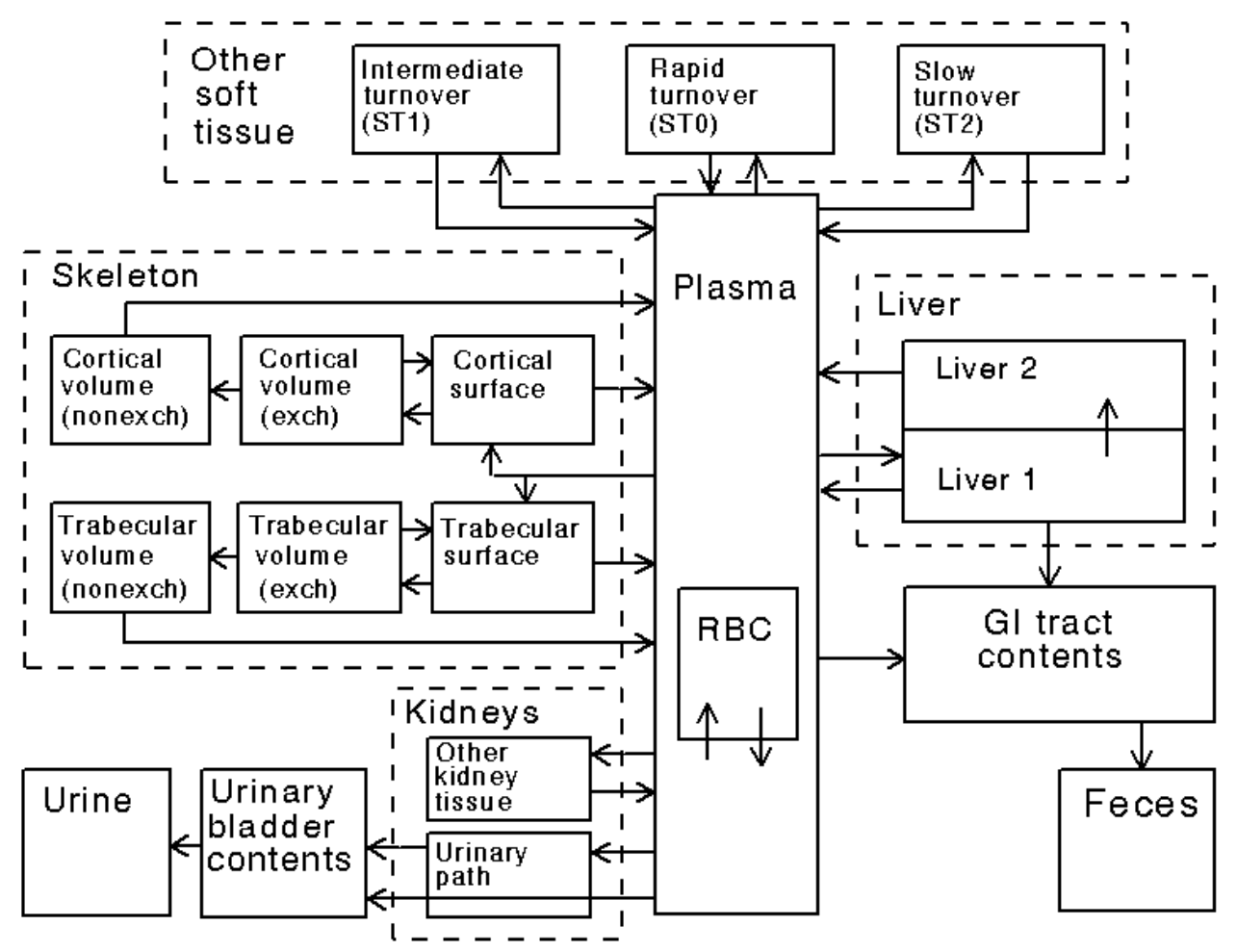

Figure 5.7. ICRP's generic model structure for calcium-like elements, introduced in ICRP Publication 67 (1993) and applied in that document or subsequent ICRP documents to calcium, strontium, barium, radium, lead, and uranium

A soft-tissue compartment ST2 with very low uptake from blood, thus having little influence on model predictions during the first $124 \mathrm{~d}$ after injection, was used to represent relatively non-exchangeable calcium resulting from dystrophic calcification of tissues such as arterial plaque and calcified nodes, a normal occurrence in humans. It has been estimated that older persons have 50-100 mg dystrophic calcium $\mathrm{kg}^{-1}$ soft tissue (Heaney, 1964). The deposition fraction and removal half-time for calcium in ST2 were set to give about $0.1 \%$ of total-body calcium in ST2 in older adults. A removal half-time from ST2 to blood plasma of $5 \mathrm{y}$ was estimated for calcium and applied to strontium, barium, and radium. Differences in accumulation of calcium, strontium, barium, and radium in soft tissues, as indicated for example by the fraction of total-body activity in soft tissues in chronically exposed persons, were modeled using element-specific transfers from plasma to ST2. 
Uptake and retention of radiostrontium in soft tissues has been measured in several human subjects, all seriously ill (Figure 5.8). For example, comparisons of ${ }^{85} \mathrm{Sr}$ and ${ }^{45} \mathrm{Ca}$ retention in soft tissues were made in several human subjects injected with these radionuclides 0.12-124 d before their deaths (Schulert et al., 1959). Data from this study indicate that soft tissues initially contain about as much strontium as bone, but the softtissue content falls off sharply after a few weeks while the bone content declines little over the first few months (Figure 5.9).

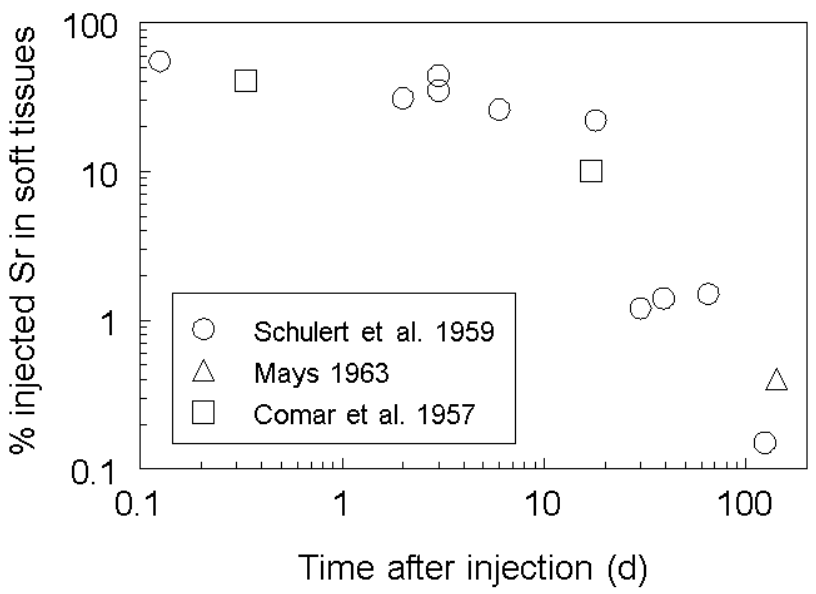

Figure 5.8. Retention of strontium in soft tissues as a function of time after intravenous injection of radiostrontium into human subjects

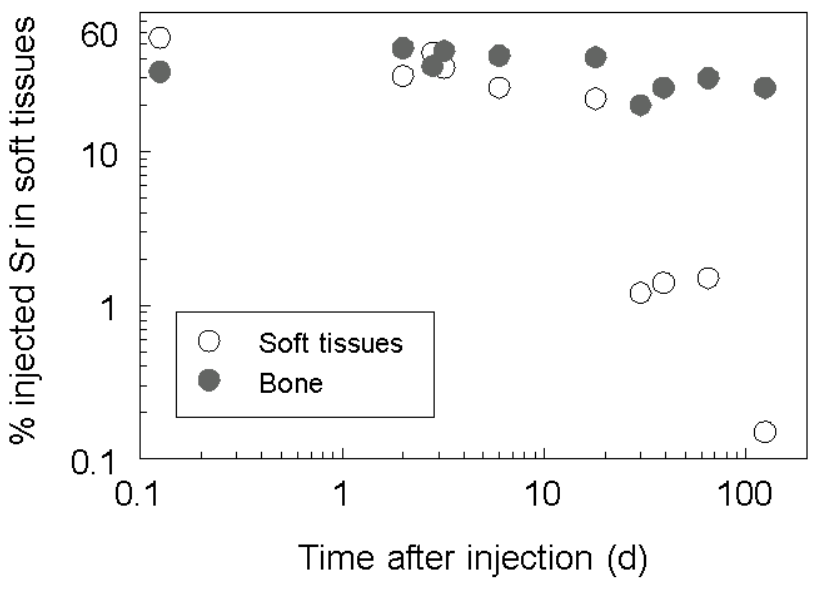

Figure 5.9. Division of activity between bone and soft tissues as a function of time after intravenous injection of radiostrontium into human subjects 
The soft-tissue retention data for strontium and comparisons with the more frequently studied element, calcium, were used to model the kinetics of strontium in soft tissues. Fractional deposition in soft tissues was assumed to be the same for strontium as for calcium. A longer removal half-time from ST1 for strontium than calcium was needed to obtain a good fit to measurements of injected ${ }^{85} \mathrm{Sr}$ in human soft tissues at about 1-4 mo after injection. Fractional deposition of strontium in the "tenacious-retention" compartment, ST2, was set to yield reasonable agreement with the estimate of Schlenker et al. (1982) that about $1 \%$ of the body's natural strontium resides in soft tissues in the average adult.

The pools ST0, ST1, and ST2 are defined on a kinetic basis but have a physical interpretation. Compartment ST0 may consist of interstitial fluids plus some rapidly exchangeable cellular calcium; compartment ST1 may be a composite of several pools with slower exchange rates, including mitochondrial calcium, cartilage calcium, and exchangeable "dystrophic" calcium (e.g., arterial plaque and calcified nodes); and compartment ST2 may be associated largely with relatively non-exchangeable dystrophic calcium that normally accumulates in the human body over a period of many years. Compartment ST2 is of limited importance for calcium but becomes more important for consideration of the heavier calcium-like elements, for which relatively immobile deposits may represent most of the soft-tissue burden at times remote from injection.

Provision is made in the generic model structure to treat the liver and kidneys separately from other soft tissues if information is available. In the case of strontium, no reason was found to distinguish the behavior in liver and kidneys from that in other soft tissues.

In the generic model, bone is divided into cortical and trabecular bone, and each of these bone types is further divided into bone surfaces and bone volume. Bone volume is viewed as consisting of two pools, one that exchanges with activity in bone surface for a period of weeks or months and a second, non-exchangeable pool from which activity can be removed only by bone restructuring processes. Activity depositing in the skeleton is assigned to bone surface, from which it is removed with an element-specific half-time on the order of days. Over a period of days a portion of the activity on bone surfaces moves to exchangeable bone volume and the rest returns to plasma. Activity leaves exchangeable bone volume with an element-specific half-time, on the order of a few weeks or months. Part of the activity leaving exchangeable bone volume is assumed to return to rapidly exchanging bone surfaces and part is assigned to non-exchangeable bone volume, from which it is assumed to be removed to plasma only by bone resorption. The rate of removal from non-exchangeable bone volume is assumed to be the (age-specific) rate of bone turnover and hence is independent of the element.

Uptake and retention of strontium by bone has been measured in terminally ill human subjects. Retention in bone can also be inferred from total-body retention following acute administration of radiostrontium, since this is nearly the same as bone retention at times greater than a few weeks after injection of strontium. Total-body retention of radiostrontium has been measured in many human subjects, including healthy subjects as 
well as hospital patients. Variability of total-body retention data is high, apparently due in large part to an age effect even during adulthood (Figure 5.10). The bone remodeling rate, which determines the level of deposition of calcium and related elements in bone, remains elevated until about age $25 \mathrm{y}$. This is reflected in measurements of total-body retention of strontium, which is generally much higher for young adults than for middleaged or elderly persons.

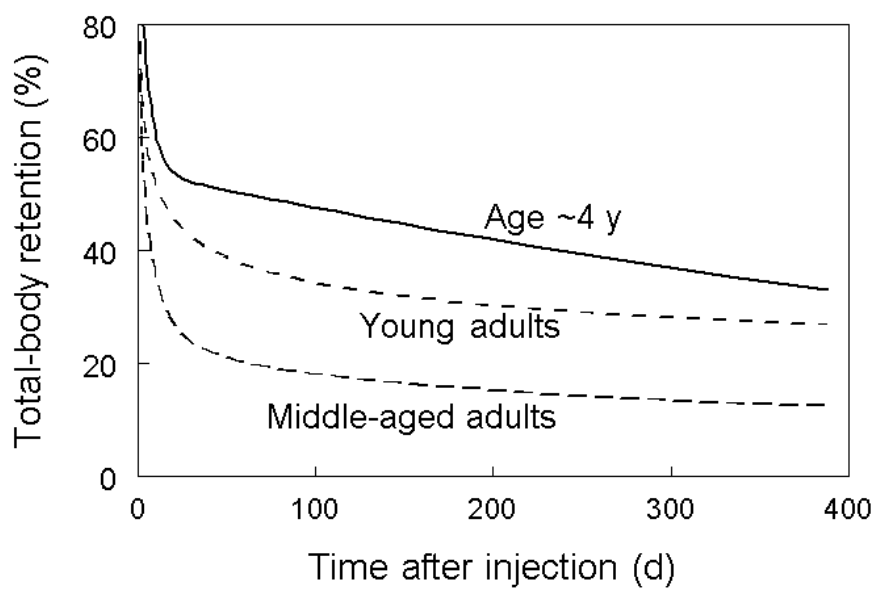

Figure 5.10. Differences with age in whole-body retention of strontium (references to original studies given in Leggett, 1992)

Data for laboratory animals indicate that fractional deposition on bone surfaces, as judged by the total skeletal content in the first few hours after injection, is similar for calcium, strontium, barium, and radium, despite the different excretion rates of these elements (Bligh and Taylor 1963; Kshirsagar et al. 1966; Domanski et al. 1969, 1980). Use of a common bone-surface deposition fraction for all four elements is also consistent with the limited human data, including: autoradiographic measurements of surface activity in bone samples taken at autopsy from subjects injected with radiocalcium at $0.6 \mathrm{~d}$ or longer before death (Riggs et al. 1971, ICRP 1973); calcium and strontium concentrations in autopsy samples of bone from subjects injected with radiocalcium and radiostrontium at 3 $\mathrm{h}$ or longer before death (Schulert et al. 1959); externally measured buildup and decline of injected radiocalcium over areas of the human body where bone activity is expected to predominate (Anderson et al. 1970, Heard and Chamberlain 1984); and external measurements of the peak skeletal content of intravenously injected ${ }^{133 \mathrm{~m}} \mathrm{Ba}$ in human subjects (Korsunskii et al. 1981). Based on these data, it is assumed that $25 \%$ of calcium, strontium, barium, or radium leaving plasma is deposited on bone surfaces of the mature adult.

The initial distribution between different bones of the skeleton and between the two bone types (cortical and trabecular) also appears to be similar for calcium, strontium, barium, and radium (Ellsasser et al. 1969, Wood et al. 1970, Liniecki 1971, Stather 1974, Lloyd et al. 1976). As discussed below, relative deposition of alkaline earth elements on 
trabecular and cortical bone surfaces is based on the estimated calcium turnover of each bone type. This approach agrees with measurements on laboratory animals (Kshirsagar et al. 1966, Norrdin and Arnold 1980). As an average over adult ages, deposition on trabecular bone is estimated to be 1.25 times that on cortical bone (Leggett et al. 1982).

The residence time on human bone surfaces has not been determined with much precision for any of the alkaline earth elements. A common removal half-time of $1 \mathrm{~d}$ is assigned to all four elements, based on the same data as considered in estimating the deposition fraction for bone surfaces, plus measurements of the early decline in whole-body retention of intravenously injected radioactive calcium, strontium, barium, or radium in human subjects (Spencer et al. 1960; Bishop et al. 1960; Heaney et al. 1964; Harrison et al. 1967; Phang et al. 1969; Carr et al. 1973; Likhtarev et al. 1975; Malluche et al. 1978; Henrichs et al. 1984; Newton et al. 1990, 1991) coupled with measurements of soft-tissue retention as described earlier. A removal half-time of $1 \mathrm{~d}$ refers to the half-time that one theoretically would observe if recycling of activity to bone surfaces could be stopped. Given the considerable amount of recycling from plasma to bone surfaces, the corresponding net or apparent half-time would be $3 \mathrm{~d}$ or more.

Parameter values for exchangeable bone volume are estimated from whole-body measurements using data for times after bone surfaces and soft tissues have largely cleared of activity but before loss from bone resorption becomes an important consideration. Based on data for human subjects injected with radioisotopes of calcium, strontium, barium, or radium (Spencer et al. 1960; Bishop et al. 1960; Heaney et al. 1964; Harrison et al. 1967; Phang et al. 1969; Carr et al. 1973; Likhtarev et al. 1975; Malluche et al. 1978; Henrichs et al. 1984; Newton et al. 1990, 1991), it was concluded that the fraction of injected activity released from bone over the intermediate term (and thus assigned to exchangeable bone volume) is roughly one-sixth of activity leaving bone surfaces for all four elements.

Removal half-times for radium, barium, strontium, and calcium from the exchangeable bone volume compartments are set at $30 \mathrm{~d}, 50 \mathrm{~d}, 80 \mathrm{~d}$, and $100 \mathrm{~d}$, respectively. These values are based in part on fits to intermediate-term retention data for human subjects injected with radioisotopes of these elements and in part on the observation that the halftime of the intermediate component for a given element appears to increase roughly in proportion to the likelihood of entering non-exchangeable sites in bone mineral, as judged either by in vitro experiments with hydroxyapatite crystals or by data on wholebody retention of alkaline earth elements in human subjects.

Discrimination between different alkaline earth elements by bone is accounted for by fractional transfer of activity from exchangeable bone volume to non-exchangeable bone volume. It is assumed, in effect, that calcium, strontium, barium, and radium are all equally likely to become temporarily incorporated in bone mineral after injection into blood but that the likelihood of reaching a non-exchangeable site in bone crystal decreases in the order calcium $>$ strontium $>$ barium $>$ radium. Fractional transfers of calcium, strontium, barium, and radium from exchangeable bone volume to deep bone volume are assumed to be $0.6,0.5,0.3$, and 0.2 , respectively, with the remainder 
(fractions $0.4,0.5,0.7$, and 0.8 , respectively) assumed to go from exchangeable bone volume to bone surface. Thus, with the probability of permanent fixation in bone crystal (i.e., removable only by bone resorption) used as a measure of discrimination, bone mineral is estimated to discriminate among the alkaline earths in the ratios calcium:strontium:barium:radium $=1.0: 0.83: 0.50: 0.33$ as an average over forming and existing bone in the adult. These values reproduce whole-body retention curves for humans injected with radioisotopes of calcium, strontium, barium, and radium, and are reasonably consistent with results of in vitro measurements. For example, under conditions approximating physiological, Neuman (1964) found that calcium incorporated into forming hydroxyapatite crystals is $65 \%$ nonexchangeable, and Stark (1968) determined discrimination ratios calcium:strontium:barium:radium $=1.0: 0.93: 0.56: 0.32$ in forming crystals. Such in vitro results have varied, however, with experimental conditions, length of aging of the crystals, and the definition of discrimination (Neuman 1964, Stark 1968).

Fractional transfer from exchangeable bone volume to deep bone volume is described above. Biological removal from deep bone volume is assumed to occur only by bone turnover. Reference age-specific bone turnover rates were taken from an earlier paper (Leggett et al., 1982). The turnover rates are based on histomorphometric measurements on human subjects as well as studies of retention of certain bone-seeking radionuclides in human subjects. Most histomorphometric measurements are on ribs and iliac crest, but there are also a few measurements for various long bones. It is assumed that there are no differences in turnover rates of cortical and trabecular bone early in life but differences gradually appear during childhood and eventually grow to about a factor of 5 in the mature adult (Leggett et al. 1982).

Clearance of alkaline earths from plasma to urine and feces has been studied in a large number of human subjects, many of them healthy. Clearance is usually expressed in terms of liters of plasma per day, but in the models for calcium and related elements, movement of activity to urine and feces is expressed in terms of deposition fractions in the urinary bladder and the GI tract contents. It is assumed that alkaline earth elements are secreted into the upper large intestine and are not reabsorbed to blood.

\subsection{Database for adult male humans}

Most of the $\mathrm{H} 1$ data for strontium summarized above represents measurements on adult males. For example, the whole-body retention curve for young adults given in Figure 5.10 is based on measurements on 44 males of median age 22 y (Likhtarev et al., 1975), and most of the data represented by the curve for middle-aged adults are for males.

Variability in whole-body retention of strontium in young adult males during the first few months after acute intake can be estimated from data of Likhtarev et al. (1975). Geometric standard deviations (GSDs) of whole-body retention at given times, the urinary excretion rate at early times, and the fecal excretion rate at $4 \mathrm{~d}$ did not exceed 1.4 for subjects receiving ${ }^{85} \mathrm{Sr}$ by injection. Calculated GSDs based on ingestion data from that study are in the range 1.6-1.7. Higher variability in retention or urinary excretion is 
to be expected in the case of ingestion due to additional variability associated with the gastrointestinal absorption fraction.

Schubert and coworkers (1967) concluded that the concentration of ${ }^{90} \mathrm{Sr}$ in bones of a large number of environmentally exposed human subjects was lognormally distributed when populations were suitably narrowed according to age, location (areas of the world), and time period. The GSD of the distribution was close to 1.6 for each sub-population considered. Since some of the variation in tissue concentrations in these subjects probably arose from variation in exposure to ${ }^{90} \mathrm{Sr}$ and hence could overstate variation in biokinetics of absorbed ${ }^{90} \mathrm{Sr}$.

\subsection{Differences with gender}

Around 1950, a plutonium production facility in Russia released large amounts of ${ }^{90} \mathrm{Sr}$ into the nearby Techa River. Shagina et al. (2003) measured whole-body content of ${ }^{90} \mathrm{Sr}$ over a 24-year period in thousands of persons living near the Techa River. They concluded that the rate of ${ }^{90} \mathrm{Sr}$ elimination is different for adult males and adult females. An increase with age in the ${ }^{90} \mathrm{Sr}$ elimination rate was indicated for adults of both genders but was much more pronounced in women than men. The results are consistent with a higher rate of bone loss in aging females than in aging males.

\subsection{Variation with age}

As illustrated in Figure 5.10, experimental and environmental studies have also revealed considerable variation with age in the biokinetics of strontium during growth. There is evidence of elevated skeletal uptake but also relatively rapid skeletal losses of strontium during periods of rapid bone growth and turnover.

A large data base related to the transfer of ${ }^{90} \mathrm{Sr}$ from food and milk to the human skeleton was developed in the 1950s and 1960s. Those data indicate that much higher transfer of ${ }^{90} \mathrm{Sr}$ from the environment to the skeleton occurs in growing children than in adults, with highest transfer occurring in infants and toddlers and a second phase of elevated uptake occurring during adolescence (Figure 5.11).

Conclusions regarding variation with age in strontium biokinetics indicated by $\mathrm{H} 1$ data are supported by studies on laboratory animals (ICRP, 1993). This illustrates the typical use of the large A1 database on strontium for purposes of biokinetic modeling, that is, as secondary, supporting information rather than as primary information. As discussed later for other cases (e.g., $\left.{ }^{106} \mathrm{Ru}\right), \mathrm{A} 1$ data is a primary source of biokinetic information for many radionulides.

\subsection{Effects of disease}

It seems likely that diseases affecting the rate of bone remodeling or bone loss could have a substantial effect on whole-body retention of strontium, but no data were found that strongly supports this hypothesis. 


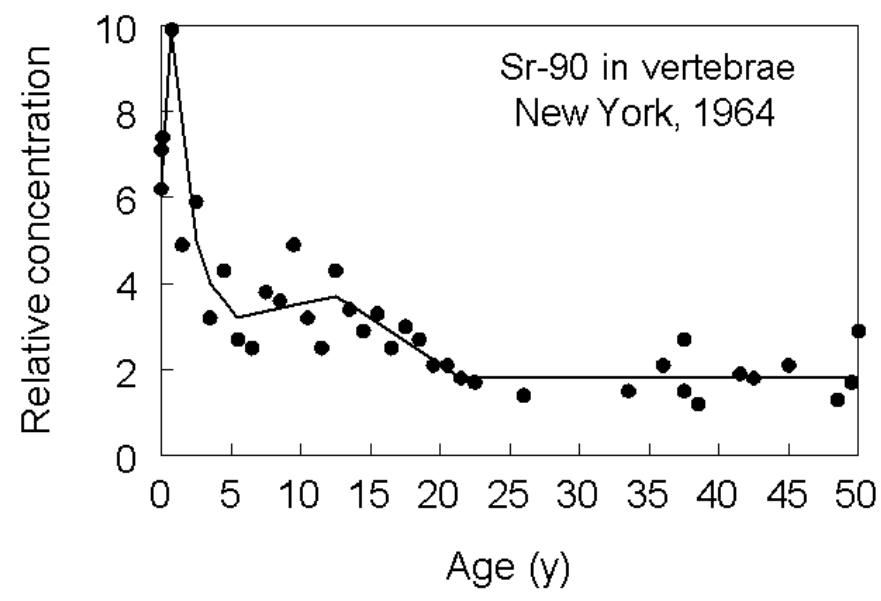

Figure 5.11. Differences with age in the concentration of ${ }^{90} \mathrm{Sr}$ in human vertebrae during a period of elevated fallout (Leggett et al., 1998)

\subsection{Biokinetics of radioactive progeny}

Strontium-90 $\left(\mathrm{T}_{1 / 2}=28.7 \mathrm{y}\right)$ decays to the short-lived radionuclide ${ }^{90} \mathrm{Y}\left(\mathrm{T}_{1 / 2}=64.1 \mathrm{~h}\right)$. Strontium and yttrium have much different chemical and biokinetic properties, although both are bone seekers. Strontium is deposited mainly on skeletal surfaces in areas of growth and remodeling but within a few days is distributed throughout the bone volume. Only a small portion of systemic strontium can be found in non-skeletal tissues within a few weeks after uptake to blood. Yttrium is deposited on resorbing and resting surfaces of bone and is likely to remain on bone surfaces for an extended period until removed to bone marrow, blood, or bone volume by bone remodeling processes. Yttrium also appears to be tenaciously retained in the liver.

Experimental evidence for laboratory animals indicates that ${ }^{90} \mathrm{Y}$ decays at its site of production in bone volume but migrates to a large extent from sites of production in soft tissues, except perhaps liver (Arnold et al., 1955; Lloyd, 1961). In experiments where rabbits were injected either with ${ }^{91} \mathrm{Y}$ or ${ }^{90} \mathrm{Sr}$ free from ${ }^{90} \mathrm{Y},{ }^{91} \mathrm{Y}$ showed greater uptake and more tenacious retention than ${ }^{90} \mathrm{Sr}$ in soft tissues. There was a secondary uptake of ${ }^{90} \mathrm{Y}$ in the liver, kidneys, and spleen due to escape from ${ }^{90} \mathrm{Sr}$ in various parts of the body. By measuring the relative activities of ${ }^{90} \mathrm{Sr}$ and ${ }^{90} \mathrm{Y}$ in various tissues of a beagle, Arnold and coworkers (1955) concluded that ${ }^{90} \mathrm{Y}$ does not escape from its parent in bone volume to any appreciable extent. 


\subsubsection{Dosimetry}

The decay schemes for ${ }^{90} \mathrm{Sr}$ and its daughter ${ }^{90} \mathrm{Y}$, including half-lives, modes of decay, and energy spectra, have been studied extensively. Both radionuclides are beta emitters. Strontium-90 emits a relatively weak beta (mean energy $=0.20 \mathrm{MeV}$ ) and ${ }^{90} \mathrm{Y}$ a relatively energetic beta (mean energy $=0.94 \mathrm{MeV}$ ). The beta radiation from both radionuclides generally will be absorbed in the organ in which it originates. Exceptions occur when source and target tissues are separated by only a few microns, such as when the source is trabecular bone and the target is trabecular marrow. In such cases the dosimetry model becomes an important source of error for ${ }^{90} \mathrm{Sr}$ dosimetry.

\subsubsection{Variability of dose per unit intake or per unit exposure}

Table 5.3 compares the estimated effective dose from ingestion of ${ }^{90} \mathrm{Sr}$ in different subgroups of the population. The estimates are based on a modification of the ICRP's age-specific biokinetic model for strontium that takes account of changes in bone remodeling rates during adulthood.

\begin{tabular}{|c|c|c|c|}
\hline \multicolumn{4}{|c|}{$\begin{array}{l}\text { Table 5.3. Estimated effective dose from ingestion of } 1 \mathrm{~Bq} \mathrm{of}{ }^{90} \mathrm{Sr} \\
\text { for different groups, relative to the estimated value for a young } \\
\text { adult male }\end{array}$} \\
\hline \multirow[t]{2}{*}{ Group } & \multirow{2}{*}{$\begin{array}{l}\text { Dose per } \\
\text { unit activity } \\
\text { ingested }\end{array}$} & \multicolumn{2}{|c|}{$\begin{array}{l}\text { Dose per unit activity } \\
\text { in food supply }\end{array}$} \\
\hline & & $\begin{array}{c}\text { Based on } \\
\text { calorie intake }\end{array}$ & $\begin{array}{c}\text { Based on } \\
\text { calcium intake }\end{array}$ \\
\hline Infants & 8.2 & 1.3 & 5.8 \\
\hline $1-y$-old & 2.6 & 0.7 & 2.0 \\
\hline 5-y-old & 1.7 & 0.9 & 1.3 \\
\hline 10 -y-old & 2.1 & 1.3 & 1.7 \\
\hline 15 -y-old male & 2.8 & 2.3 & 3.1 \\
\hline 15-y-old female & 3.0 & 1.9 & 2.1 \\
\hline 25 -y-old male & 1.0 & 1.0 & 1.0 \\
\hline 25 -y-old female & 1.2 & 0.8 & 0.8 \\
\hline 65 -y-old male & 0.9 & 0.7 & 0.7 \\
\hline 65-v-old female & 0.9 & 0.5 & 0.5 \\
\hline
\end{tabular}

\subsubsection{Existing uncertainty assessments for intake of ${ }^{90} \mathrm{Sr}$}

Uncertainties in the biokinetics of ${ }^{90} \mathrm{Sr}$ in humans and in doses from intake of ${ }^{90} \mathrm{Sr}$ have been addressed in several studies (NRC-CEC, 1998; Bogen et al., 1997; Leggett et al., 1998; EPA, 1999; Harrison et al., 2001; Apostoaei, 2002; Apostoaei and Miller, 2004). The general conclusion is that the doses from intake of ${ }^{90} \mathrm{Sr}$ are reasonably well understood, but an unstated assumption appears to be that ${ }^{90} \mathrm{Sr}$ is ingested in relatively soluble form. 
Harrrison et al. (2001) concluded from a literature review that high-confidence intervals for average fractional absorption of strontium from the gastrointestinal tract are relatively narrow compared with other incompletely absorbed elements: $0.1-0.4$ for adults, $0.1-0.5$ for age $10 \mathrm{y}$, and $0.15-0.75$ for infants. Apostoaei (2002) concluded that the uncertainty in the gastrointestinal absorption fraction for strontium in adults is well represented by a lognormal distribution with GSD of 1.44 and $95 \%$ confidence interval 0.11 to 0.46 . Apostoaei's conclusion apparently refers to uncertainty for an individual and results primarily from inter-individual variability, while Harrison and coworkers are referring to the uncertainty in the central value. On the basis of a literature review and limited sensitivity analysis, Leggett et al. (1998) concluded that the ICRP's systemic biokinetic model for strontium has moderate to high reliability (uncertainty factor of about 3 ) with regard to predicted cumulative activity of ${ }^{90} \mathrm{Sr}$ in bone volume.

Apostoaei and Miller (2004) used parameter uncertainty analysis to investigate uncertainties in tissue doses from ingestion of ${ }^{90} \mathrm{Sr}$. They collected data on strontium biokinetics in humans, on the energy deposited in each organ, and on the masses of the organs. They derived distributions from these data which they interpreted as representing uncertainties in parameter values, and they attempted to identify correlations between parameters. Monte Carlo methods were used to propagate uncertainties through the biokinetic and dosimetric models. According to the investigators:

"Throughout this work, the uncertainties represent, to the extent possible, interindividual variation of the given parameter. In those cases when data were insufficient to clearly describe the inter-individual variability, the uncertainty describes the range of possible values obtained by professional judgment."

The ICRP's current systemic biokinetic model for strontium (Figure 5.7) was used. Organ-specific dose factors were calculated for different cases: (1) assuming no agedependency in parameter values and assuming that ${ }^{90} \mathrm{Y}$ produced in vivo has the same kinetics as ${ }^{90} \mathrm{Sr}$; (2) using age-dependent parameter values and assuming that ${ }^{90} \mathrm{Y}$ produced in vivo has the same kinetics as ${ }^{90} \mathrm{Sr}$; (3) assuming no age-dependency in parameter values but assigning the ICRP's biokinetic model for $\mathrm{Y}$ to ${ }^{90} \mathrm{Y}$ produced in vivo. According to Apostoaei and Miller, "The analysis of data for deriving model parameter distributions follows the logic used by Leggett (1992) to select the default parameter values for the ICRP biokinetic model for ${ }^{90} \mathrm{Sr}$ ". They explained this as follows: Initial selections were made for individual parameter values based on the best available evidence for the pathway considered, and then, due to the interdependence of the parameter values, repeated tuning of initial selections was performed until satisfactory fits to available human data were obtained. Because the analysis by Apostoaei and Miller was an uncertainty analysis rather than model construction, "the parameters were derived as probability distribution functions, and comparisons with the experimental data consisted of two important aspects: a) the predicted central value should reproduce the observed central tendency of the data, and b) the range of the predicted values at any moment in time should cover inter-individual variation of the data for that moment." The investigators concluded that the uncertainties in ingestion dose coefficients for ${ }^{90} \mathrm{Sr}$ are described by lognormal distributions. The largest derived uncertainties are for bone 
surface $(\mathrm{GSD}=2.6)$ and bone marrow $(\mathrm{GSD}=2.4)$. They concluded that the contributions to organ dose uncertainties decreased in the following order of importance: variability in biokinetic model parameters $>$ mass of target organ $>$ energy deposition in target organ.

The methods of the analysis indicate that the results should apply to uncertainty in dose estimates for an individual, which would overstate the uncertainty in dose as defined in the present report. Also, the results of Apostoaei and Miller may overstate variability in the biokinetics of strontium for the adult as defined by the ICRP. The ICRP defines the adult to be $25 \mathrm{y}$ old or greater in the case of strontium biokinetics because of the sizable differences in kinetics between very early adulthood and the greater portion of adulthood (Figure 5.10).

\subsection{Cobalt-60 (Level 3)}

\subsubsection{Overview}

The biokinetic database for cobalt includes results of controlled studies on human subjects (H1), follow-up studies of accidentally exposed workers (H1), and data for different animal species (A1). There have been several human studies of the biokinetics of inhaled cobalt in the respiratory tract and of gastrointestinal uptake, but results vary considerably with the form of cobalt taken into the body and conditions of the study. Whole-body retention of inorganic cobalt in adults can be estimated with reasonably high confidence from results of controlled human studies (H1), but information on the internal distribution comes mainly from studies on laboratory animals (A1). The animal data cannot be extrapolated to humans with high confidence because of apparent species differences in the behavior of cobalt. For example, the long-term retention component is considerably larger in human subjects than other studied species, and the difference cannot be explained by metabolic rate or body size. Also, although $\mathrm{H} 1$ data on hepatic retention of cobalt are limited, it appears that the liver is a more important long-term repository for cobalt in humans than in other studied species. Model development is complicated by the fact that environmental cobalt may have substantially different biokinetic properties from the inorganic forms of cobalt generally used in experimental studies. Age-specific data for cobalt are available only for rats, which is not a preferred species for age-specific modeling. With regard to dose estimates for internally deposited ${ }^{60} \mathrm{Co}$, the fact that there is substantial cross-irradiation of tissues by the high-energy photon emissions from ${ }^{60} \mathrm{Co}$ tends to offset uncertainties in the systemic distribution of cobalt to some extent. Nevertheless, dose estimates for internally deposited ${ }^{60} \mathrm{Co}$ seem much less reliable than estimates for internally deposited ${ }^{90} \mathrm{Sr}$ or ${ }^{137} \mathrm{Cs}$.

\subsubsection{Biokinetics}

\subsubsection{Physiological analogues}

Cobalt is an integral component of vitamin $\mathrm{B}_{12}$, but humans are exposed to many forms of cobalt other than $\mathrm{B}_{12}$. Cobalt shows some chemical and physiological resemblance to 
iron but is not a close physiological analogue of iron. Absorption of cobalt in the small intestine appears to involve a transport mechanism that is partly shared with iron (Pollack et al. 1965, Schade et al. 1970a, 1970b). Iron deficiency leads to increased absorption of both iron and cobalt (Pollack et al. 1965). Iron absorption is reduced when it is administered together with cobalt (Schade et al. 1970a, 1970b), but absorption of cobalt is not diminished in iron-replete human subjects, indicating that competitive displacement of the elements is directed against iron but not against cobalt (Valberg et al., 1969, 1972).

\subsubsection{Behavior in the respiratory tract}

Information on the behavior of inhaled cobalt in the respiratory tract has been reviewed in ICRP Publication 71 (1995) and recently by an ICRP task group (to be published in an upcoming ICRP document). Recent experimental data on inhaled cobalt were also discussed in an ICRP guidance document on applications of the HRTM (ICRP, 2002a).

Extensive data are available on the fate of inhaled cobalt in the respiratory tract, in part due to frequent occupational exposures and in part because ${ }^{60} \mathrm{Co}$, with its easily measured gamma emissions, has been used frequently in experimental studies of the behavior of inhaled particles. Workers have been exposed to cobalt as metal dusts, oxides or soluble salts.

Although numerous studies have been carried out on the toxicity of inhaled cobaltcontaining alloys, the data do not reveal the clearance kinetics of cobalt. Data obtained from diamond polishers and experimental data for rats suggest long-term retention in the lungs indicative of Type $\mathrm{M}$ or $\mathrm{S}$ behavior after inhalation of alloys.

Studies in several animal species have shown that most of the cobalt deposited in the lungs after inhalation as chloride or nitrate is rapidly absorbed, but a few percent of the inhaled material is absorbed over several months. Overall, the absorption is consistent with assignment to Type F.

Detailed studies have been conducted of the lung clearance kinetics of various physical forms of cobaltosic oxide $\left(\mathrm{Co}_{3} \mathrm{O}_{4}\right)$, including comparisons of clearance from the lungs of human volunteers, baboon, dog, guinea pig, rat, hamster and, mouse. Lung retention was longer in humans and baboons than in the other species. The inhaled material was designed to be moderately soluble, and it was determined that the rate of absorption was reasonably consistent with Type $\mathrm{M}$ parameter values. When the test material was selected to be less soluble, absorption in baboons and dogs was consistent with assignment to Type S. Studies of lung retention in workers after accidental inhalation of radioactive cobalt oxide aerosols show slow removal from the lungs, broadly consistent with Type $\mathrm{S}$ parameter values.

Highly insoluble polystyrene or fused aluminosilicate particles (FAP) labeled with ${ }^{60} \mathrm{Co}$ have been used as relatively insoluble particles in inhalation studies of biokinetics and radiation effects. After inhalation of either material, a small fraction of the inhaled 
activity is rapidly absorbed, but the rest is retained within the particles and is absorbed slowly, from polystyrene at a rate of about $0.00005 \mathrm{~d}^{-1}$ and from FAP at a rate of about $0.0005 \mathrm{~d}^{-1}$. These values are broadly consistent with the long-term rate of $0.0001 \mathrm{~d}^{-1}$ for particle removal from AI assumed for Type S material.

\subsubsection{Fractional uptake from the gastrointestinal tract}

Information on gastrointestinal uptake of cobalt was reviewed by Harrison et al. (2001). Except where a specific study is cited, the following summary is taken from that review.

Human data on uptake are highly variable. Three studies involving relatively large study groups ( $\mathrm{n}=37$ in one study) have been performed using $\mathrm{CoCl}_{2}(\mathrm{H} 1)$. The results indicate mean absorption on the order of 0.02-0.08 with administration of tracer quantities, increasing to about $0.25-0.30$ with added carrier. In rats, only a few percent of cobalt is absorbed after ingestion of inorganic forms, but $30-70 \%$ is absorbed after ingestion in vitamin $B_{12}$. An absorption fraction of about 0.05 was estimated for ${ }^{60} \mathrm{Co}$ ingested in crab meat by human subjects.

Christensen (1995) studied gastrointestinal absorption of cobalt in adult males and females. Soluble cobalt (chloride) was absorbed much more than insoluble (oxide) cobalt, and females absorbed much more than males.

Studies on rats indicate that GI uptake of cobalt is highly elevated during the first few weeks of life, being nearly complete for organic forms and up to an order of magnitude greater than in adults for inorganic forms. Absorption was also elevated in newborn guinea pigs but lower than in rats. Human data are available from a balance study of girls aged between 6 and 10 years in which absorption was estimated as 0.57 . This is higher than most reported values for absorption of cobalt in adult humans, but absorption estimates based on balance data generally involve sizable uncertainties.

The ICRP's current absorption values for cobalt are 0.1 for adults, 0.3 for children from 1 $\mathrm{y}$ to 15 years of age and 0.6 for 3 month-old infants.

\subsubsection{Systemic biokinetics}

\subsection{ICRP model}

The ICRP's current systemic biokinetic model for cobalt (ICRP, 1993) does not have a physiologically meaningful structure. As in the model structure shown in Figure 5.1 for cesium, the cobalt model depicts one-directional movement of activity from blood to tissues to excretion pathways. The model divides systemic cobalt between liver and all other tissues combined, and activity in each of these repositories is assigned three retention components. Cobalt is assumed to leave blood with a half-time of $0.5 \mathrm{~d}$, with a fraction 0.5 going to excretion pathways, 0.05 going to the liver, and 0.45 uniformly distributed among the other organs and tissues. Fractions $0.6,0.2$, and 0.2 of activity translocated from blood to tissues are assumed to be retained with biological half-times 
of 6,60 , and $800 \mathrm{~d}$, respectively, and to be lost directly to excretion pathways. Activity moving to excretion pathways is divided between the urinary bladder contents and the upper large intestine contents in the ratio 6:1. The biokinetics of systemic cobalt is assumed to be independent of age.

\subsection{Summary of biokinetic data on absorbed cobalt}

Whole-body retention or other aspects of cobalt biokinetics have been investigated by external measurement in several human subjects after accidental intake or administration of inorganic forms (Taylor, 1959; Smith et al., 1972; Letourneau et al., 1972; Beleznay and Osvay, 1994; Jansen et al., 1996). The collective data indicate biological removal of perhaps three-fourths of the injected amount from the body over the first few days, another $10-15 \%$ over a period of weeks or months, and the remainder with a half-time on the order of $2 \mathrm{y}$. Whole-body measurements in two subjects over a period of 1000 days after intravenous injection of ${ }^{60} \mathrm{CoCl}_{2}$ indicated that retention could be described by a four component exponential function with the following average biological half-times (and component sizes): 0.54 days (44\%); 5.6 days (32\%); 63 days (13\%) and 800 days (11\%) (Smith et al., 1972). Based on whole-body counting of 16 male subjects over a $1-y$ period following intravenous injection of ${ }^{58} \mathrm{CoCl}_{2}$, Letourneau and coworkers (1972) determined a five-component retention function with half-times (and component sizes) 0.25 days (36\%); 1.8 days (24\%); 8 days (19\%) 47 days (13\%) and 608 days (9\%).

Beleznay and Osvay (1994) measured retention of ${ }^{60} \mathrm{Co}$ between 10 and $1850 \mathrm{~d}$ in six workers who accidentally inhaled ${ }^{60} \mathrm{Co}$ aerosols during manipulation of a high-activity source. A retention component of 25-78 d was interpreted as activity leaving the deep lungs. A long-term component of retention determined in five of the workers followed for extended periods was interpreted as the slowest component of systemic retention of cobalt. The half-time of the long-term component varied from about $500 \mathrm{~d}$ to about $1200 \mathrm{~d}$ and averaged about $900 \mathrm{~d}$ in the five subjects.

Plasma clearance of cobalt following intravenous injection of ${ }^{60} \mathrm{Co}$-chloride is rapid (Smith et al., 1972). A substantial portion of the uptake to blood is rapidly excreted in urine. Taylor (1959) measured the urinary excretion of ${ }^{57} \mathrm{Co}$ in 13 normal human subjects ( 9 male, 4 female) during the first 24 hours after intravenous injection of cobalt glycinate; the average excretion was $34 \%$ of the injected activity with a range of $23 \%$ to $47 \%$. There was no significant difference between the first day urinary excretion in males and females. Measurements of the excretion of ${ }^{60} \mathrm{Co}$ in the urine and feces of six subjects studied over 2 to 8 days showed that the greater proportion of the cobalt was eliminated by the kidneys, $22 \%$ being eliminated on the first day; fecal excretion during the study periods ranged from 5\% to $30 \%$, with an average of about $15 \%$. Taylor (1959) also reported a urinary to fecal excretion ratio of about $6: 1$ in one subject studied over a period of three days.

Smith et al. (1972) concluded from external measurements that the amount of cobalt present in the liver may represent roughly one-fifth of the total-body content as an average over 0-1000 d (Smith et al. 1972). More recently, Jansen et al. (1996) used 
positron emission tomography to study the retention of ${ }^{55} \mathrm{Co}$ in the liver in two male volunteers and concluded that $\sim 50 \%$ of ${ }^{55} \mathrm{Co}$ intravenously injected as ${ }^{55} \mathrm{CoCl}_{2}$ deposited in the liver.

The biokinetics of cobalt has been studied in a number of different animal species, including mice, rats, hamsters, guinea pigs, dogs, monkeys, and baboons (A1). These studies provide information on the time-dependent distribution of absorbed cobalt as well its retention time in the body. However, extrapolation of the data to man is fraught with uncertainty due to apparent species differences in the biokinetics of cobalt. For example, even though long-term retention times do not appear to vary greatly among species, the portion of administered activity that is retained for a long period is much greater in human subjects than in non-human species that have been studied (Figure 5.12). Also, comparison of external data for human subjects with findings for laboratory animals suggests that the liver is a much more important long-term repository for cobalt in man than in the studied animal species.

Characterization of the typical biokinetics of cobalt is further complicated by evidence that its biological behavior depends on the chemical form taken into the body. For example, marked differences were seen in uptake and tissue distribution of ${ }^{60} \mathrm{Co}$ in rats gavaged with inorganic ${ }^{60} \mathrm{CoCl}_{2}$ or with a homogenate of fish in which ${ }^{60} \mathrm{Co}$ had been biologically incorporated (Inaba et al., 1982).

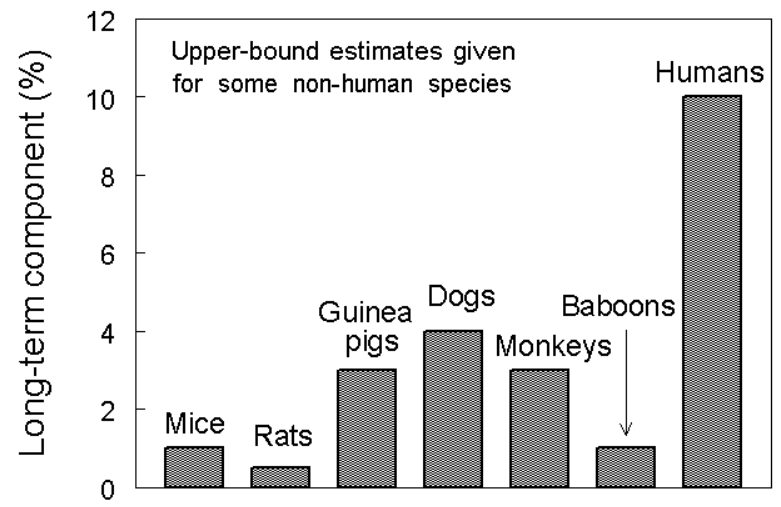

Figure 5.12. Species comparison of the size of the long-term retention component for inorganic cobalt (after Figure 8 of Leggett et al., 1998)

\subsection{Database for adult male humans}

Most of the H1 data for cobalt described above are for adult males. For example, a study of the biokinetics of intravenously administered ${ }^{58}$ Co by Letourneau et al. (1972) involved 16 adult males, a study by Smith et al. (1972) of the behavior of intravenously 
administered ${ }^{60} \mathrm{Co}$ involved 11 adult males. A related study of the behavior of orally administered ${ }^{60} \mathrm{Co}$ by Smith et al. (1972) involved 12 adult males and one adult female.

\subsection{Differences with gender}

Christensen (1995) observed that urinary cobalt was higher in females than in males after ingestion of cobalt and hypothesized that this was due to higher GI uptake of cobalt by females, possibly associated with iron deficiency. Comparative data on the systemic biokinetics of cobalt in adult males and females are limited but do not indicate important differences with gender. For example, the measured long-term retention half-time in a female subject was within the range determined in male subjects (Figure 5.12). After intravenous injection of ${ }^{57} \mathrm{Co}$ glycinate into nine adult males and four adult females, no significant difference between the first day urinary excretion in males and females (Taylor, 1959).

\subsection{Variation with age}

It is assumed in ICRP Publication 67 (1993) that the systemic biokinetics of cobalt is independent of age. This assumption was made partly on the weak basis that no evidence for age dependence was seen in a study on rats, and in part due to lack of a physiological basis for deriving age-specific parameter values.

\subsection{Effects of disease}

No data were found regarding effects of disease on the biokinetics of cobalt.

\subsection{Biokinetics of radioactive progeny}

Cobalt-60 does not have radioactive progeny.

\subsubsection{Dosimetry}

Decay properties of ${ }^{60} \mathrm{Co}$ including half-life, modes of decay, and energy spectra are well understood. It produces two gamma rays with energies of $1.17 \mathrm{MeV}$ and $1.33 \mathrm{MeV}$ and is also a beta emitter with maximum beta energy of $1.55 \mathrm{MeV}$. The dosimetric model is not an important source of uncertainty in dose estimates for ${ }^{60} \mathrm{Co}$.

\subsubsection{Variability of dose per unit intake or per unit exposure}

Adult females may receive higher tissue doses than adult males per unit ingestion of ${ }^{60} \mathrm{Co}$ due to elevated GI uptake of cobalt in adult females. Elevated absorption of cobalt in infants is suggested by animal data (A1) but has not been confirmed by human studies. Elevated GI uptake of cobalt in children and adolescents, particularly adolescent girls, may occur due to an apparent relation between cobalt and iron absorption. Also, results 
of a balance study on girls up to age $10 \mathrm{y}$ indicate relatively high uptake of cobalt. For the most part, however, the age-specific dose coefficients for ${ }^{60}$ Co provided in ICRP documents are based on assumptions rather than real information.

\subsubsection{Existing uncertainties assessments for ${ }^{60} \mathrm{Co}$}

Based on a critical review of the literature, Harrison et al. (2001) assigned the following high-confidence intervals for average fractional absorption of environmental cobalt from the GI tract: $0.02-0.2$ for adults, $0.03-0.5$ for age $10 \mathrm{y}$, and $0.04-0.8$ for infants. On the basis of a literature review and limited sensitivity analysis, Leggett et al. (1998) concluded that the ICRP's systemic biokinetic model for cobalt has relatively low reliability with regard to predicted cumulative activity of ${ }^{60} \mathrm{Co}$ in the liver. Apostoaei et al. (1998) used parameter uncertainty analysis to assess uncertainties in dose coefficients for ingestion of ${ }^{60} \mathrm{Co}$ on the basis of the ICRP's simplistic model structure and derived an uncertainty factor, which they defined as the ratio of the 97.5 th to 50th percentiles of the derived "probability distribution", of about 8 for all tissues. Sensitivity analyses performed during the development of FGR13 (EPA, 1999) suggest moderate to high uncertainty, depending on the tissue, in dose estimates from inhalation or ingestion of ${ }^{60} \mathrm{Co}$.

\subsection{Ruthenium-106 (Level 4)}

\subsubsection{Overview}

The biokinetics of ruthenium is not as well understood as that of cobalt, for example, due mainly to a paucity of $\mathrm{H} 1$ data. The behavior of ruthenium has been studied in several animal species, but a consistent picture of the fate of inhaled, ingested, or absorbed ruthenium has not developed. Dose estimates for ${ }^{106} \mathrm{Ru}$ are sensitive to gaps in the biokinetic information.

\subsubsection{Biokinetics}

\subsubsection{Physiological analogues}

Ruthenium is in Group VIII of the periodic chart, also referred to as the platinum metals. In addition to ruthenium and platinum, this group includes iron, osmium, cobalt, rhodium, iridium, nickel, and palladium. Available data do not reveal a close physiological analogue of ruthenium in this group. Ruthenium and rhodium have similar chemical properties and appear to have broadly similar biokinetics in rats, but comparative data for these two elements are limited and the database for rhodium is too sparse to be of much use in modeling the biokinetics of ruthenium. 


\subsubsection{Behavior in the respiratory tract}

Information on the behavior of inhaled ruthenium in the respiratory tract has been reviewed in ICRP Publication 71 (1995) and recently by an ICRP task group (to be published in an upcoming ICRP document). The following summary of inhalation data is abstracted from those reviews.

In seven persons who accidentally inhaled ${ }^{103} \mathrm{RuO}_{2}$ the effective half-life of the radioactivity in the chest was found to be 26.6 days. In five men who accidentally inhaled ${ }^{106} \mathrm{RuO}_{2}$ over a brief period, the mean biological half-time for retention in the chest was $206 \pm 30 \mathrm{~d}$ (range, 174-428 d). In beagles, inhaled ${ }^{106} \mathrm{RuO}_{2}$ was avidly retained in the lungs, with an estimated biological half-life of $\sim 2000$ days. Based on these studies and data for rats, the retention pattern for inhaled ${ }^{106} \mathrm{RuO}_{2}$ seems broadly consistent with Type S. Results from an in vitro dissolution study of samples of particles released from the Chernobyl accident indicate that ruthenium associated with irradiated fuel fragments should be assigned to default Type $\mathrm{M}$ in lieu of more specific information.

Ruthenium may exist in gas or vapor form as well as in particulate form. Measurements on a person accidentally exposed to ruthenium tetroxide vapor $\left(\mathrm{RuO}_{4}\right)$ indicated that deposition occurred only in the extrathoracic airways, where the compound was subsequently retained. Observations of the fate of inhaled ${ }^{106} \mathrm{RuO}_{4}$ in dogs and rats indicate that deposition was primarily in the nasopharyngeal and tracheobronchial regions and that most of the deposited activity was lost in feces over a period of days.

\subsubsection{Fractional uptake from the gastrointestinal tract}

The following summary of the database on GI uptake of ruthenium was abstracted from a review by Harrison et al. (2001).

Measurements of the absorption of ruthenium in male volunteers after ingestion of chloro-complexes of $\mathrm{Ru}(\mathrm{III})$ and $\mathrm{Ru}(\mathrm{IV})$ or absorption from contaminated clams gave values of about 0.01 . Values for nitrosyl $\mathrm{Ru}(\mathrm{III})$ were about three times greater. Results from studies of the absorption of ${ }^{106} \mathrm{Ru}$ administered as the chloride to mice, rats, rabbits, guinea pigs, chickens, cats, dogs and monkeys, including values for fasted animals, indicate fractional uptake in the range of $0.03-0.06$. Values for ${ }^{106} \mathrm{Ru}$ administered as the oxide to rats and rabbits were in the range of $0.003-0.03$. Absorption of ruthenium administered to rats and rabbits as a nitrato-nitrosyl complex was 0.06 and 0.13 , respectively. A value of 0.04 was reported for the absorption of ruthenium administered to rats as a nitro-nitrosyl. (Nitrato and nitro- complexes of nitrosyl ruthenium are formed during dissolution in nitric acid in the reprocessing or $\mathrm{U}$ fuels.)

Data on rats and mice indicate that fractional uptake of ruthenium from the GI tract may be as high as $0.07-0.08$ in newborn animals. The ICRP's currently recommended absorption values for ruthenium ingested by members of the public are 0.1 for infants and 0.05 for ages $1 \mathrm{y}$ or greater, both of which are cautiously high values. 


\subsubsection{Systemic biokinetics}

\subsection{ICRP model}

The ICRP's current systemic biokinetic model for ruthenium (ICRP, 1989, 1993) does not have a physiologically meaningful structure but depicts one-directional movement of activity from blood to tissues to excretion pathways. Activity is assumed to be removed from blood with a half-time of $0.3 \mathrm{~d}$, with $15 \%$ going to excretion pathways. The remainder is assumed to be uniformly distributed throughout all organs and tissues of the body and is divided into three retention components: $35 \%$ is removed to excretion pathways with a biological half-time of $8 \mathrm{~d}, 30 \%$ with a half-time of $35 \mathrm{~d}$, and $20 \%$ with a half-time of $1000 \mathrm{~d}$. Activity assigned to excretion pathways is divided between the urinary bladder contents and the upper large intestine contents in the ratio 4:1. The systemic biokinetics of ruthenium is assumed to be independent of age.

\subsection{Summary of biokinetic data on absorbed ruthenium}

Direct information on the systemic biokinetics of ruthenium in the human body (H1) is sparse. Yamagata et al. $(1969,1971)$ measured whole-body retention of ruthenium in a healthy adult male who ingested different chemical forms of ${ }^{103} \mathrm{Ru}\left(\mathrm{T}_{1 / 2}=39.3 \mathrm{~d}\right)$ or ${ }^{106} \mathrm{Ru}\left(\mathrm{T}_{1 / 2}=373.6 \mathrm{~d}\right)$ on different occasions. Results of the study using ${ }^{103} \mathrm{Ru}$ suggested the possibility of two retention components for absorbed activity, one with a biological half-life of $2.3 \mathrm{~d}$ and one with a half-time of about $30 \mathrm{~d}$. The estimate for the early component is not particularly useful for modeling purposes because it could reflect mainly unabsorbed activity. The longer-term behavior of ruthenium in the body could not be determined with much confidence due to the short half-life of ${ }^{103} \mathrm{Ru}$. Results from a later study on the same subject using ${ }^{106} \mathrm{Ru}$ suggested a retention component with halftime of about $9 \mathrm{~d}$ and a second component with half-life $32 \mathrm{~d}$. At longer times, the estimated biological "half-time" lengthened with the period of observation: $81 \mathrm{~d}$ based on observations in the period 40-80 d after intake, $122 \mathrm{~d}$ at 80-150 d after intake, $158 \mathrm{~d}$ at 150-350 d after intake, and $385 \mathrm{~d}$ at 350-660 d after intake.

Veronese et al. (2001) studied the blood kinetics of ruthenium in three healthy volunteers using the stable isotope ${ }^{101} \mathrm{Ru}$. The results depend on the mode of intake (intravenously injected or ingested) and the chemical form injected and add little to our understanding of the systemic biokinetics of ruthenium.

Due to the limitations of data on the biokinetics of ruthenium human subjects $(\mathrm{H} 1)$, the ICRP based its systemic biokinetic model on observations of the behavior of ruthenium in laboratory animals (A1). The studied animal species include mice, rats, guinea pigs, rabbits, cats, dogs, and monkeys (Burykina, 1962; Furchner et al., 1971; Stara et al., 1971; ICRP, 1989). Reported long-term retention half-times range from about $200 \mathrm{~d}$ to about $1600 \mathrm{~d}$ and show no trend with body mass. The longest "half-time" observed in the human subject studied by Yamagata (1971) is within this broad range of values (Figure 5.13). 
Data on the systemic distribution of ruthenium comes mainly from studies on rodents and suggest a somewhat uniform distribution, the main exception being an elevated concentration in the kidneys in the early weeks after injection. The ICRP's biokinetic model for ruthenium was based mainly on data for mice, rats, monkeys, and dogs (Furchner et al. 1971), which were interpreted as indicating that the systemic biokinetics of ruthenium is reasonably independent of species.

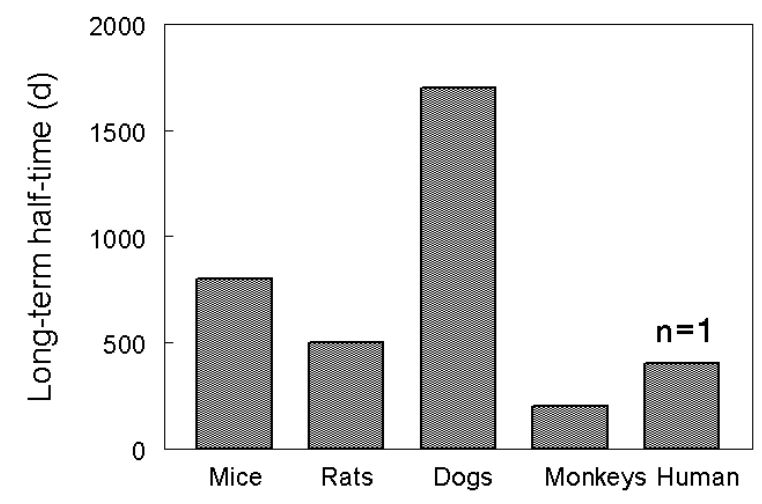

Figure 5.13. Species comparison of long-term retention of ruthenium in the total body

\subsection{Database for adult male humans}

With the exception of limited information on the blood kinetics of ruthenium, the only $\mathrm{H} 1$ data on the systemic behavior of ruthenium is the information described earlier for an adult male who ingested different forms of ruthenium on different occasions (Yamagata et al., 1969, 1971).

\subsection{Differences with gender}

No information was found on differences with gender in the biological behavior of ruthenium.

\subsection{Variation with age}

No information was found on age related changes in the systemic biokinetics of ruthenium. As discussed earlier, GI uptake of ruthenium appeared to be elevated in newborn rodents.

\subsection{Effects of disease}

No data were found relating to potential effects of disease on the biokinetics of ruthenium. 


\subsection{Biokinetics of radioactive progeny}

Ruthenium-106 decays to the short-lived ${ }^{106} \mathrm{Rh}(\mathrm{T} 1 / 2=29.9 \mathrm{~s})$. It seems unlikely that

${ }^{106} \mathrm{Rh}$ migrates to any appreciable extent from ${ }^{106} \mathrm{Ru}$ in view of the chemical similarity of ruthenium and rhodium and the short half-life of the ${ }^{106} \mathrm{Rh}$, but there is no direct information on the fate of ${ }^{106} \mathrm{Rh}$ produced in vivo.

\subsubsection{Dosimetry}

The dosimetry model for the ${ }^{106} \mathrm{Ru}$ chain does not appear to be an important source of error in dose estimates from intake of ${ }^{106} \mathrm{Ru}$. Decays of the short-lived daughter ${ }^{106} \mathrm{Rh}$ represent over $99 \%$ of the energy deposited in tissues. The decay scheme for ${ }^{106} \mathrm{Rh}$ is well established. It is a beta and gamma emitter with half life of $29.9 \mathrm{~s}$. Its maximum beta energy is $3.54 \mathrm{MeV}$. Its mean photon energy is $0.60 \mathrm{MeV}$.

\subsubsection{Variability of dose per unit intake or per unit exposure}

Available data provide little insight into the variability of dose in the population from intake of ${ }^{106} \mathrm{Ru}$. Age-specific dose coefficients for ${ }^{106} \mathrm{Ru}$ are provided in ICRP documents but are based on assumptions and suggestive data for rodents rather than real information for humans or preferred animal models.

\subsubsection{Existing uncertainties assessments for intake of ${ }^{106} \mathrm{Ru}$}

\subsubsection{Summary of published assessments}

Harrison et al. (2001) concluded from a literature review that high-confidence intervals for average fractional absorption of ruthenium from the gastrointestinal tract are 0.0050.1 for adults, $0.005-0.15$ for age $10 \mathrm{y}$, and $0.005-0.2$ for infants. On the basis of a literature review and limited sensitivity analysis, Leggett et al. (1998) concluded that the ICRP's systemic biokinetic model for ruthenium has relatively low reliability with regard to predicted cumulative activity of ${ }^{106} \mathrm{Ru}$ in the total body. Apostoaei et al. (1998) used parameter uncertainty analysis to assess uncertainties in dose coefficients for ingestion of

${ }^{106} \mathrm{Ru}$ on the basis of the ICRP's simplistic model structure for ruthenium and derived uncertainty factors (ratio of 97.5 th to 50th percentiles of the probability distribution) of about 3 for the intestines, 6 for stomach, and 9 for other tissues. Sensitivity analyses performed during the development of FGR13 (EPA, 1999) suggest that dose from inhalation or ingestion of ${ }^{106} \mathrm{Ru}$ cannot be determined with much confidence and that moderately high confidence can be placed in external dose coefficients for ${ }^{106} \mathrm{Ru}$.

\subsubsection{A preliminary assessment based on comparison of alternate models}

The authors of the present report performed limited calculations to examine the sensitivity of dose coefficients for ${ }^{106} \mathrm{Ru}$ to uncertainties in the biokinetics of ruthenium in the adult. A comparison was made of dose estimates for intravenously injected ${ }^{106} \mathrm{Ru}$ 
generated by substantially different but equally plausible biokinetic models for ruthenium. This procedure is illustrated here for three such models, called Models A, B, and $\mathrm{C}$, developed by different modelers. Model A is the ICRP's current biokinetic model for this element, described earlier. Model B, shown in Figure 5.14, was developed by Runkle et al. (1980) as a fit to their measurements of ${ }^{106} \mathrm{Ru}$ in tissues and excreta of rats exposed to ${ }^{106} \mathrm{RuO}_{4}$ by inhalation or ingestion.

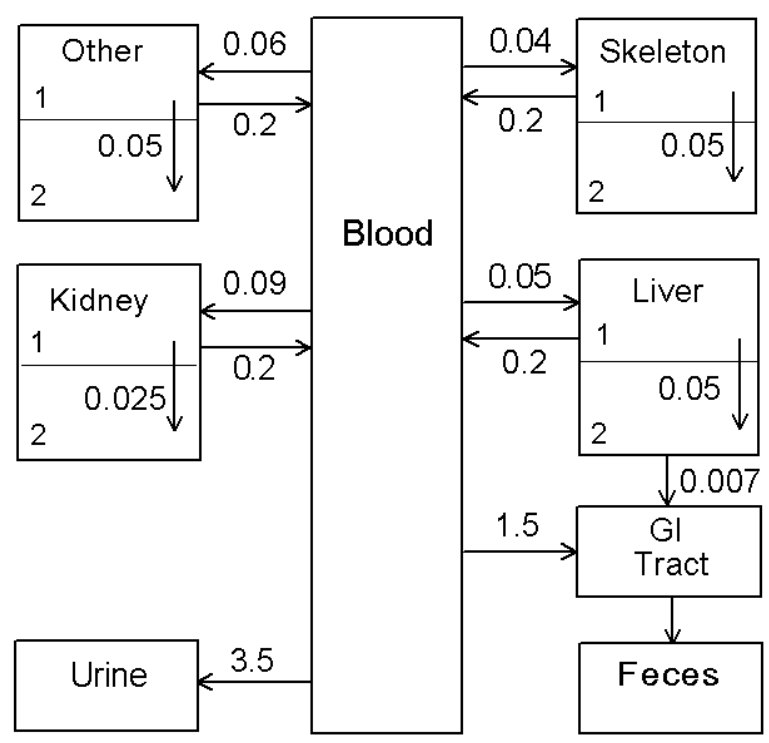

Figure 5.14. Systemic biokinetic for ruthenium developed by Runkle et al. (1980) (Model B in text) based on data for rats exposed to ${ }^{106} \mathrm{RuO}_{4}$ by inhalation or ingestion

Model C has a structure similar to that of Model B but differs substantially from Model B with regard to rates of movement of activity between compartments. Transfer rates for Model C were based on the broadly consistent whole-body retention data for dogs (Furchner et al., 1971) and a human subject (Yamagata et al., 1971) and on the distribution of systemic activity observed in guinea pigs (Burykina, 1962). Activity is assumed to be removed from blood with a half-time of $0.25 \mathrm{~d}$, with $20 \%$ depositing in the urinary bladder contents, $5 \%$ in the small intestine contents, $20 \%$ in liver (in a compartment called Liver 1), $10 \%$ in the kidneys (Kidney 1), 35\% in other soft tissues (Other 1), and $10 \%$ on bone surface (Skeleton 1). Activity leaves Liver 1, Kidney 1, Other 1, and bone surface with a half-time of $5 \mathrm{~d}$. Of activity leaving Liver 1 or Other 1, $90 \%$ returns to blood and $10 \%$ moves to a long-term compartment of the same tissue (Liver 2 or Other 2, respectively). Of activity leaving Kidney 1, 98\% returns to blood and $2 \%$ moves to a long-term compartment in the kidneys (Kidney 2). Of activity leaving bone surface, $75 \%$ returns to blood and $25 \%$ moves to bone volume (Skeleton 2). Activity moves from Liver 2 to the small intestine contents with a half-time of $100 \mathrm{~d}$, from Kidney 2 to Kidney 1 with a half-time of 100 d, from Other 2 to Other 1 with a halftime of $500 \mathrm{~d}$, and from bone volume to bone surface with a half-time of $1000 \mathrm{~d}$. 
Dose coefficients for ${ }^{106} \mathrm{Ru}$ based on the three different models are compared in Table 5.4. Relative to the ICRP's model for ruthenium, Model C yields substantially higher estimates of dose equivalent to liver, kidneys, and skeletal tissues, generally lower estimates for other tissues, and a reasonably similar (30\% greater) effective dose. Compared with the ICRP's model for ruthenium, Model B yields lower estimates of dose equivalent to all tissues except the kidneys, and estimates of the effective dose are reduced by a factor of 6 .

These comparisons of predictions of three equally plausible systemic models for ruthenium indicate the relatively large uncertainties in dose per unit activity of ${ }^{106} \mathrm{Ru}$ reaching blood. This analysis does not address the uncertainties associated with the behavior of inhaled ${ }^{106} \mathrm{Ru}$ in the respiratory tract or the level of absorption of inhaled or ingested ${ }^{106} \mathrm{Ru}$ to blood, and it provides an indication of only minimal uncertainties for absorbed ${ }^{106} \mathrm{Ru}$. Thus, it is evident that dose estimates for inhaled or ingested ${ }^{106} \mathrm{Ru}$ involve sizable uncertainties.

\begin{tabular}{lccc}
\hline $\begin{array}{l}\text { Table 5.4. Comparison of injection dose coefficients for } \\
\text { based on three different biokinetic models for ruthenium described in the text }\end{array}$ \\
\hline & $\begin{array}{c}\text { Dose coefficient } \\
\text { based on Model } A\end{array}$ & $\begin{array}{c}\text { Ratio of dose } \\
\text { coefficients }\end{array}$ & $\begin{array}{c}\text { Ratio of dose } \\
\text { coefficients }\end{array}$ \\
& $(\mathrm{Sv} \mathrm{Bq})$ & $B: A$ & $C: A$ \\
Tissue & $2.9 \mathrm{E}-08$ & 0.13 & 4.5 \\
\hline Bone surfaces & $2.9 \mathrm{E}-08$ & 0.020 & 0.54 \\
Stomach wall & $2.9 \mathrm{E}-08$ & 0.062 & 0.57 \\
Small intestine wall & $3.3 \mathrm{E}-08$ & 0.24 & 0.62 \\
Upper large intestine wall & $4.1 \mathrm{E}-08$ & 0.53 & 0.73 \\
Lower large intestine wall & $2.9 \mathrm{E}-08$ & 2.5 & 6.7 \\
Kidneys & $2.9 \mathrm{E}-08$ & 0.11 & 4.5 \\
Liver & $2.9 \mathrm{E}-08$ & 0.12 & 3.4 \\
Red marrow & $3.4 \mathrm{E}-08$ & 0.16 & 0.58 \\
Urinary bladder wall & $2.9 \mathrm{E}-08$ & $\sim 0.02$ & $\sim 0.55$ \\
Other tissues & $3.0 \mathrm{E}-08$ & 0.16 & 1.3 \\
Effective dose & &
\end{tabular}

\subsection{Californium-252 (Level 5)}

\subsubsection{Overview}

The biokinetic database for californium is sketchier than that for ruthenium. The only H1 data found for californium consists of limited measurements on two persons who accidentally inhaled ${ }^{252} \mathrm{Cf}$ and a person who accidentally inhaled ${ }^{249} \mathrm{Cf}$ together with its parent, ${ }^{249} \mathrm{Bk}$. Excretion data for the person exposed to ${ }^{249} \mathrm{Cf}$ are not consistent with any of the default absorption types used by the ICRP. Information on GI uptake comes from measurements on rats. Information on the systemic biokinetics of californium comes from studies on laboratory animals, including dogs. The dosimetric model for ${ }^{252} \mathrm{Cf}$ involves potentially large errors associated with the model of spontaneous fission and the radiation weighting factors for alpha particles and neutrons. For these reasons, dose 
estimates for internally deposited ${ }^{252} \mathrm{Cf}$ may involve even larger errors than estimates for internally deposited ${ }^{106} \mathrm{Ru}$.

\subsubsection{Biokinetics}

\subsubsection{Physiological analogues}

Biokinetic studies of californium in laboratory animals indicate that its behavior is qualitatively similar to that of other transuranium elements. Among the frequently studied transuranics, americium appears to be its closest physiological analogue. The microscopic distribution of californium in soft tissues of beagles 1-3 wk after intravenous injection of a citrate solution was found to be similar to that of americium (Taylor et al., 1972). The gross distribution of californium in the skeleton, expressed as the percentage of skeletal californium in a given bone, is similar to that of americium (Lloyd et al., 1972). The microscopic distribution of californium in the skeleton is also similar to that of americium in rats, with heaviest deposits on the trabeculae of the primary spongiosa and on epiphyseal and metaphyseal trabeculae (Durbin 1973).

\subsubsection{Behavior in the respiratory tract}

A case of accidental inhalation of a mixture of ${ }^{249} \mathrm{Cf}$ and its parent, ${ }^{249} \mathrm{Bk}$, by a chemist was studied by external measurements and excretion analysis over the first year after intake (Rundo and Sedlet, 1973). The inhaled material was ignited before intake and was presumably highly insoluble. Except for an initially rapid clearance via feces, the urinary and fecal excretion rate of both radionuclides increased with time for 2-3 months after intake and then declined. This pattern differs from the monotonically decreasing rates of transfer from lungs to blood and excretion in urine and feces depicted in models of the ICRP(1994a, 2002a).

In another case, a chemist and an analyst inhaled airborne ${ }^{252} \mathrm{Cf}$ while attempting to reprocess a medical source (Poda and Hall, 1975). Approximately $1 \mu \mathrm{g}^{252} \mathrm{Cf}_{2} \mathrm{O}_{3}$ was released when the end plug of the inner capsule was sheared during the removal of the outer capsule. Both persons left the work area when an alpha air monitor sounded shortly after the accident. An initial survey indicated contamination in the nostrils and on the clothing of the analyst (Subject 1), but no contamination was detected on the chemist (Subject 2). Both persons were treated with chelates. A rapid renal excretion of $\mathrm{Cf}$ was observed for the first 24-hour period in each subject but may have been strongly affected by DTPA treatment. Total urinary excretion of ${ }^{252} \mathrm{Cf}$ during the first month by the chemist was 1-2 orders of magnitude lower than that for the analyst. Urinary excretion patterns for the two subjects are shown in Figure 5.15, where excretion has been normalized to the percentage of the first day's excretion for the respective individual.

\subsubsection{Fractional uptake from the gastrointestinal tract}

Gastrointestinal absorption of californium has been measured in rats administered

${ }^{252} \mathrm{Cf}\left(\mathrm{NO}_{3}\right)_{3}$ intragastrically. Absorption is in the range $0.01-0.1 \%$ depending on the 
method of estimation (ICRP, 1979). The ICRP assumes absorption of $0.05 \%$ of ingested californium in an adult, which is consistent with values determined for plutonium, americium, and curium in human subjects (H2) as well as with the limited animal data for californium (A1).

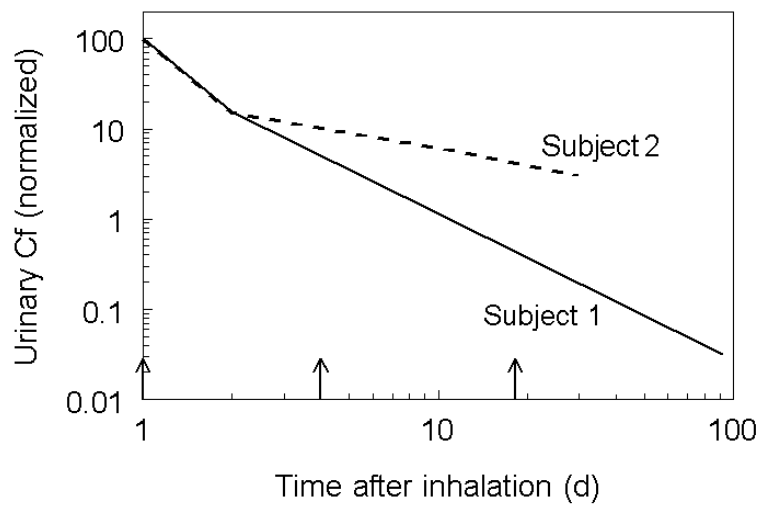

Figure 5.15. Observed patterns of urinary excretion of ${ }^{252} \mathrm{Cf}$ following acute inhalation. DTPA administered on Days 1, 4, and 18 (arrows) (data normalized to individual's Day 1 excretion)

\section{$\underline{\text { 5.5.2.4. Systemic biokinetics }}$}

\subsection{ICRP model}

The ICRP's current systemic biokinetic model for californium was introduced in ICRP Publication 30 (1979) and extended to a bioassay model in Publication 68 (1994). The model does not have a physiologically meaningful structure but depicts one-directional movement of activity from blood to tissues to excretion pathways. It is assumed that californium leaves blood with a half-time of $0.25 \mathrm{~d}$, with $65 \%$ depositing in the skeleton, $25 \%$ in the liver, $0.011 \%$ in ovaries, and $0.035 \%$ in testes. The rest $(9.954 \%)$ is promptly excreted. The removal half-time is $50 \mathrm{y}$ for skeleton, $30 \mathrm{y}$ for liver, and infinite for gonads. Activity lost by prompt excretion or through biological removal from tissues is equally divided between the urinary bladder contents for removal in urine and the upper large intestine contents for removal in feces.

\subsection{Summary of biokinetic data on absorbed californium}

The biokinetics of californium has been studied in mice, rats, Chinese and Syrian hamsters, and beagles (Parker et al., 1962; Mewhinney et al., 1971, 1972; Lloyd et al., 1971, 1972, 1974, 1976; Smith, 1972; Atherton and Lloyd, 1972; Bruenger et al., 1972; Stevens and Bruenger, 1972; Taylor et al., 1972; Durbin, 1973; Graham et al., 1978). Species differences have been observed. For example, Mewhinney et al. (1972) found significant differences in the behavior of ${ }^{252} \mathrm{Cf}$ in rats and Chinese hamsters over $64 \mathrm{~d}$ following intraperitoneal injection of the citrate complex, including lower uptake of 
activity by the liver and kidneys and higher uptake by the skeleton in rats and much faster removal from the liver in rats (Table 5.5). The behavior of californium in beagles receiving ${ }^{249} \mathrm{Cf}$ or ${ }^{252} \mathrm{Cf}$ by intravenous injection (Lloyd et al., 1971, 1972) was broadly similar to that in the hamster with regard to uptake and retention in major repositories. The fecal to urinary excretion ratio was much higher in rats than in dogs, probably due to a higher rate of biliary secretion of californium.

\begin{tabular}{|c|c|c|c|}
\hline \multicolumn{4}{|c|}{$\begin{array}{l}\text { Table 5.5. Species differences in the early distribution } \\
\text { of }{ }^{252} \text { Cf injected as citrate (Mewhinney et al., 1972; } \\
\text { Lloyd et al., 1972; Durbin, 1973) }\end{array}$} \\
\hline \multirow[b]{2}{*}{ Tissue or excreta } & \multicolumn{3}{|c|}{$\%$ injected activity at $7-8 \mathrm{~d}$} \\
\hline & Hamster & Rat & Dog \\
\hline Kidney & 2.9 & 1.2 & 0.9 \\
\hline Liver & 25.6 & 3.5 & 19.2 \\
\hline Skeleton & 25.3 & 65.7 & 44.1 \\
\hline Whole body & 66.3 & 69.3 & 78.3 \\
\hline Urine & -- & 7.8 & 15.1 \\
\hline Feces & -- & 11.0 & 6.9 \\
\hline
\end{tabular}

Measurements on rats and mice indicate a biological half-time for the whole body on the order of 2 y (400-1000 d). This reflects primarily skeletal retention on these animals because the removal half-time from the liver is short and other soft tissues do not retain much californium. In dogs or hamsters, whole-body retention of californium reflects tenacious retention of in both the liver and skeleton. For the beagle, half-times of $8.5 \mathrm{y}$ and $4.2 \mathrm{y}$ have been estimated for the whole body and liver, respectively.

Observed species differences in the retention time of californium in the liver is consistent with a pattern seen for other transuranic elements. That is, certain mammalian species show rapid removal of transuranics from the liver, while others show extremely slow removal. For example, rats, tree shrews, macaque monkeys, and baboons show rapid loss of plutonium from the liver, with half-times of 4-200 d, while another set of adult animals with an overlapping range of body weights, including hamsters, dogs, pigs, and humans, show tenacious retention of plutonium in the liver, with half-times measured in years or decades (Taylor, 1984).

In the skeleton, californium appears to be deposited most heavily about the trabeculae of the primary spongiosa and on epiphyseal and metaphyseal trabeculae. In soft tissues of dogs, relatively high concentrations are found in the hepatic cells of the liver, the glomeruli of the kidney, the interfollicular region of the thyroid, the cartilaginous tissues of the lung, and in the smaller arterioles of most organs. Intense but scattered "hot spots" were found in the renal papillae and the submucosa of the bronchioles. Except for deposition in hepatic cells, most of the deposition sites in soft tissues were extracellular, associated with connective tissue. 
The beagle is expected to be a reasonable laboratory model for the biokinetics of californium in humans due to qualitative similarities in the biokinetics of other transuranics (plutonium, americium, and curium) in dogs and humans, particularly for the liver and skeleton. In extrapolating biokinetic data for californium from beagles to humans, species differences in rates of apparently pertinent physiological processes must be taken into account. For example, the residence time of californium in bone may be substantially greater in adult humans than in adult beagles due to a slower rate of bone turnover in humans.

\subsection{Database for adult male humans}

The cases of accidental inhalation of californium isotopes described earlier involved adult males. No other H1 data for californium were found.

\subsection{Differences with gender}

No information was found on differences with gender in the biological behavior of californium.

\subsection{Variation with age}

No information was found on age related changes in the systemic biokinetics of californium. Based on findings for other transuranics in laboratory animals, it is expected that fractional deposition would be greater in the growing skeleton than the mature skeleton.

\subsection{Effects of disease}

No data were found relating to potential effects of disease on the biokinetics of californium.

\subsection{Biokinetics of radioactive progeny}

The decay chain beginning with ${ }^{252} \mathrm{Cf}$ includes 17 radionuclides, but the radioactive progeny of ${ }^{252} \mathrm{Cf}$ can be neglected in dose estimates from intake of pure ${ }^{252} \mathrm{Cf}$ due to the extremely long half-life of the next chain member, ${ }^{248} \mathrm{Cm}\left(\mathrm{T}_{1 / 2}=3.5 \times 10^{5} \mathrm{y}\right)$.

\subsubsection{Dosimetry}

The decay scheme for ${ }^{252} \mathrm{Cf}$ is reasonably well understood with regard to half-life, modes of decay, and decay energies. However, the dosimetric model used to convert ${ }^{252} \mathrm{Cf}$ decays to equivalent doses to tissues involves potentially large errors. These errors arise mainly from a simplistic representation of spontaneous fission and uncertain RBEs for alpha particles and neutrons. 
The dosimetry model takes account of the fission decay fraction, the number of neutrons emitted per fission, and the intensities and energies for spontaneous fission fragments, neutrons, beta particles, prompt gamma rays, and delayed gamma rays. The assumption is made, however, that the energies associated with all of these sources are emitted at the time and point of alpha emissions from ${ }^{252} \mathrm{Cf}$, which is a considerably simplified depiction of actual events.

The ICRP currently applies a radiation weighting factor of 20 for alpha radiation and 520 for spontaneous fission neutron radiation, depending on the energy. These weighting factors are representative values (perhaps on the cautiously high side of central estimates) of RBEs that have been estimated from studies of human populations or laboratory animals exposed to neutrons or alpha emitting radionuclides, in vitro data, and theoretical considerations (NCRP, 1990).

Ranges of estimated RBE for alpha particles or neutrons are wide, depending on both the biological system and the observed endpoint (NCRP, 1990; EPA, 1999). The uncertainty in the RBE estimated from an individual study also is usually large, primarily due to the uncertainty in extrapolation of low-LET data to low doses. At relatively high doses, the effectiveness of alpha emitters has been found to be 15 to 50 times that of beta emitters for the induction of bone sarcomas, liver chromosome aberrations, and lung cancers (NCRP, 1990). Since the LET of secondary protons produced by fission neutrons in living tissue is comparable to that for alpha particles, data on the RBE of fission neutrons provides ancillary information relevant to the estimation of alpha particle RBE. Experimental data as well as theoretical considerations indicate that the neutron RBE depends on energy. Where the dose response data on carcinogenic endpoints are adequate to derive an estimate, fission neutrons have been found to have an RBE of 6-60 times that of low dose gamma rays (NCRP, 1990). Overall, experimental data for solid tumor induction with alpha particles and fission neutrons suggest a central value for RBE between 10 and 30 and a range of roughly 5 to 60 for the RBE relative to low-dose, lowLET radiation (NCRP, 1990; EPA, 1999).

The problem also arises that the effective RBE may vary with cancer type or cancer site. Site-specific cancer risk estimates for alpha particles have been calculated using human epidemiological data on low-LET radiation such as in the Atomic Bomb Survivor Study and laboratory data on the RBE of high-LET radiation compared to a reference low-LET radiation (NCRP, 1990). There is evidence that the RBE of 20 commonly applied to alpha particles is much too high with regard to radiogenic leukemia. In FGR13 (EPA, 1999), an RBE of 1 was applied to alpha-particle irradiation of red marrow.

Another important source of uncertainty regarding dosimetry of alpha emitters such as ${ }^{252} \mathrm{Cf}$ is the potentially heterogeneous distribution of the radionuclide or the target cells, or both, in tissues. Because alpha particles have an extremely short range, the fraction of alpha emissions reaching radiosensitive cells is difficult to estimate if either the cells or the alpha emitter is not fairly uniformly distributed. Tissues known or thought to have heterogeneous distributions of radiosensitive cells include the lungs, bone, bone marrow, and gonads. 
As an illustration, the hematopoietic stem cells of bone marrow are known to be radiosensitive and to be heterogeneously distributed. It is not feasible to model the distribution of these cells in much detail, and the ICRP assumes simply that the cells are uniformly distributed within the marrow space of trabecular bone. If the sensitive cells were actually located more than $10 \mu \mathrm{m}$ from the bone mineral surface, the fraction of alpha energy emitted by a radionuclide on the surface of trabecular bone that is absorbed by these cells would be no more than about 0.3 for alpha energy in the range $5-8 \mathrm{MeV}$, compared with the value 0.5 assumed by the ICRP. For an alpha emitter uniformly distributed throughout the mineral of trabecular bone, the fraction of emitted energy reaching the red marrow depends on the energy of the alpha particle. Calculations for bone-volume-distributed alpha emitters ranging in energy from 5 to $8 \mathrm{MeV}$ indicate that the absorbed fraction in the marrow space ranges between 0.041 and 0.087 , which bracket the value of 0.05 recommended by the ICRP. If the sensitive cells were located more than $10 \mu \mathrm{m}$ from the bone mineral surface, the relevant absorbed fraction would be reduced to $0.015-0.055$. Additional uncertainties in the dosimetry arise from the fact that the distribution of alpha emitters such as ${ }^{252} \mathrm{Cf}$ on bone surfaces or within bone marrow is poorly characterized. For example, ${ }^{252} \mathrm{Cf}$ might accumulate in regions of bone marrow that contain relatively few radiosensitive cells, in which case alpha emissions from ${ }^{252} \mathrm{Cf}$ in bone marrow would present little risk.

\subsubsection{Variability of dose per unit intake or per unit exposure}

There is no direct information on the variability of dose in the population from exposure to ${ }^{252} \mathrm{Cf}$. On the basis of animal data for transuranium elements, it is expected that gastrointestinal absorption of ${ }^{252} \mathrm{Cf}$ would be elevated in infants, and that the deposition ratio skeleton:liver would be greater during growth than after maturity.

\subsubsection{Existing uncertainties assessments for intake of ${ }^{252} \mathrm{Cf}$}

\section{$\underline{\text { 5.5.5.1. Published assessments }}$}

No previous uncertainty assessments concerning the biokinetics of californium or dose from intake of ${ }^{252} \mathrm{Cf}$ were found.

\subsubsection{Comparison with an alternate model}

It is evident that the ICRP's current systemic biokinetic model for californium (described above) is not physiologically meaningful and does not fully reflect available information on the biokinetics of californium. In fact, the model does not reflect specific data for californium but is based on the assumption that californium behaves the same as plutonium (ICRP, 1979). The ICRP's systemic biokinetic model for plutonium has been updated several times since the appearance of ICRP Publication 30, but the model for californium remains the same as originally formulated, although explicit excretion pathways were added in ICRP Publication 68 (1994). 
A more detailed model based on current data for californium has been proposed (Leggett, 2001). The ICRP's generic model for bone-surface-seeking radionuclides is applied (Figure 5.16). Parameter values are based on the relatively detailed information on californium biokinetics in laboratory animals, particularly dogs, and the comparative biological behavior of californium and americium. The rationale is that there is relatively good information on the behavior of americium in human subjects and comparative data on americium and californium in animals. It seems reasonable to use the ICRP's model for americium as a starting point for modeling the biokinetics of californium and adjust the parameter values for americium as indicated by comparative data on americium and californium in beagles.

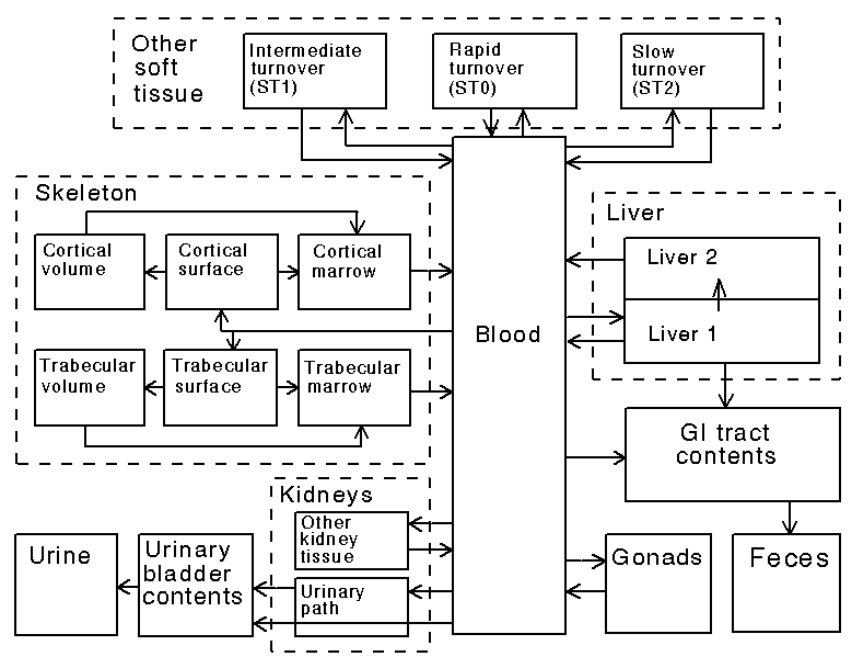

Figure 5.16. Structure of the proposed model for californium (based on the ICRP's generic model structure for bone-surface-seeking radionuclides - ICRP, 1993)

The following adjustments to the ICRP's systemic biokinetic model for americium were made (Leggett, 2001): the removal half-time from blood is $1 \mathrm{~h}$, compared with $30 \mathrm{~min}$ for americium; deposition in liver plus skeleton is $70 \%$ (i.e., $70 \%$ of activity leaving the circulation), compared with $80 \%$ for americium; the division between liver and skeleton is $20 \%-50 \%$, compared with $50 \%-30 \%$ for americium; deposition in urinary bladder contents is $11 \%$, compared with $7 \%$ for americium; deposition in the contents of the GI tract is $6 \%$, compared with $1.3 \%$ for americium; deposition in the urinary path (Kidneys 1 ) is $2 \%$ and in other kidney tissues (Kidneys 2 ) is $1 \%$, compared with $2 \%$ and $0.5 \%$, respectively, for americium; the removal half-time from Kidneys 2 to blood is $5 \mathrm{y}$, compared with $500 \mathrm{~d}$ for americium; the removal half-time from the intermediate-term soft-tissue compartment to blood is $100 \mathrm{~d}$, compared with $50 \mathrm{~d}$ for americium. Otherwise, the biokinetics of californium is assumed to be the same as that of americium.

The proposed model for californium was applied to estimate the rate of urinary excretion of californium and dose per unit intake of californium isotopes. Predicted urinary excretion rates following introduction of californium to blood are compared in Figure 
5.17 with predictions of the current ICRP model. Predictions of the ICRP model are 1-2 orders of magnitude lower than those of the proposed model from a few days to a few weeks after uptake of californium to blood.

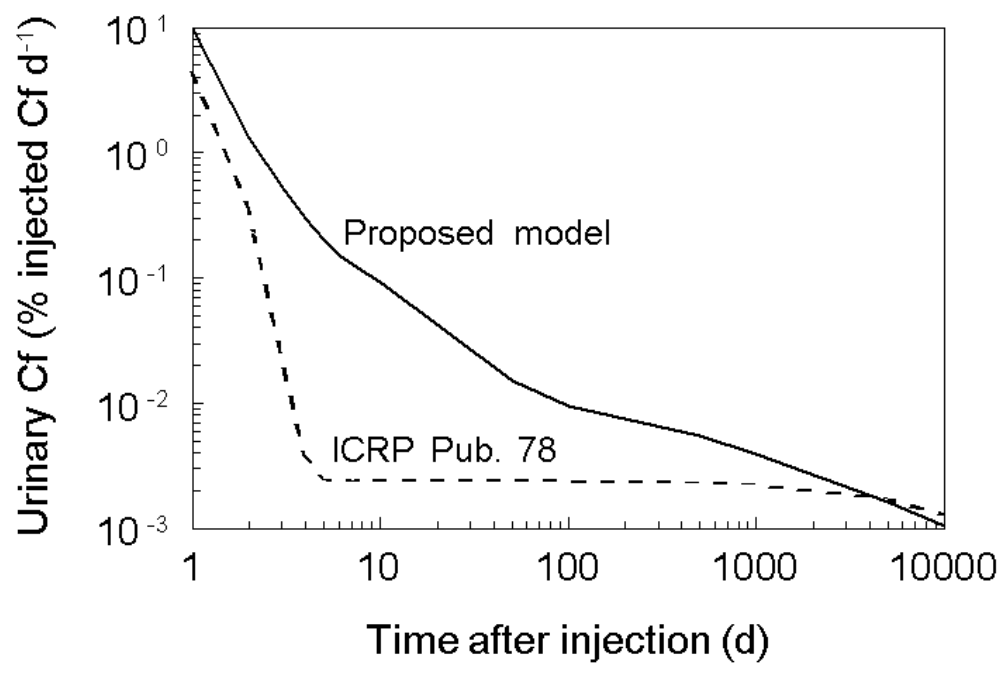

Figure 5.17. Comparison of predictions of urinary californium based on the systemic biokinetic model for californium given in ICRP Publication 68 and Publication 78 (1997) and a proposed model (Leggett, 2001), assuming intravenous injection of californium at time 0 


\section{DEMONSTRATION OF DIFFERENT LEVELS OF POTENTIAL ERROR IN EXTERNAL DOSE COEFFICIENTS}

This section discusses the basis for the external dose coefficients given in FGR12. For selected radionuclides, potential errors associated with applications of those dose coefficients are examined.

\subsection{The external dose model of FGR12}

The dose coefficients in FGR12 (EPA, 1993) are based on idealized exposure geometries and a mathematical phantom representing a reference adult male standing in an upright position at the air-ground interface. The calculation of external dose rates from radionuclides distributed in air, soil, or ground surfaces was divided into two steps: (1) calculation of the photon radiation field incident on the surface of the body and (2) calculation of organ dose rates due to a body surface source. With the exposure geometry considered, an error in an external dose coefficient for a specific radionuclide, exposure scenario (e.g., standing on a contaminated ground surface), and tissue would reflect the error in the estimate of the radiation field incident upon the body and the error in the estimated dose rate to specific tissues due to a body surface source. The latter errors could arise in part from the simplified representation of the human body as a mathematical phantom. In the adult phantom used in FGR12, the size and shape of an organ or tissue are approximated by a three-dimensional figure such as a portion of a sphere, cylinder, or cone, that can be represented by a relatively simple mathematical expression involving positional variables $\mathrm{x}, \mathrm{y}$, and $\mathrm{z}$.

Checks of external radiation fields derived in FGR12 against actual measurements and alternate theoretical methods suggest that, for photon energies above $200 \mathrm{keV}$, the methods of FGR12 generally are accurate within a few percent for the simple geometries considered. Also, estimated organ dose rates due to a body surface source are expected to be accurate within a few percent in most cases for photon energies above $200 \mathrm{keV}$. Errors in estimated external dose increase with decreasing photon energy and can be greater than $100 \%$ for photon energies of a few tens of $\mathrm{keV}$.

Voxel phantoms have been used to investigate variability in external dose and provide a check on the accuracy of external dose estimates based on mathematical phantoms. A voxel phantom is based on computed tomographic data for a real person, with each organ, tissue, or fluid represented as a set of points describing its volume and position as determined in the scan. A voxel phantom is typically based on a living adult who is undergoing a scan for medical purposes but sometimes is based on a cadaver.

Zankl et al. (2002) used seven adult male and female voxel models to examine intersubject variability of tissue doses for external whole body irradiation by photons of energies between $10 \mathrm{keV}$ and $10 \mathrm{MeV}$. The idealized geometries considered were broad parallel photon beams in anterior-posterior, posterior-anterior, left- and right-lateral direction and a full 360 degree rotation around the body length axis. Dose differences 
between the different voxel models were below $30 \%$ for some organs and geometries for photon energy between 60 and $200 \mathrm{keV}$ but were $100 \%$ or more in some cases due to differences in stature and individual anatomical details. According to Zankl and coworkers, the inter-individual differences in body size and organ geometry could amount to several hundred per cent for low photon energies. Comparison with estimates based on mathematical models indicated differences of several tens of per cent for some organs.

\subsection{Quality of the decay data}

The quality of current nuclear decay data was discussed briefly in previous sections in connection with conversion from internally deposited activity to tissue dose for selected radionuclides. For most radionuclides the quality of the nuclear decay data is expected to be a minor source of uncertainty. Some exceptional cases are discussed in Section 7.

The same nuclear decay data and anthropomorphic phantoms applied to internally deposited radionuclides are used to derive dose coefficients for external exposure. In particular, the decay database described in ICRP Publication 38 (1993) and mathematical phantoms used to develop the inhalation and ingestion dose coefficients currently recommended by the ICRP were used to derive the external dose coefficients tabulated in FGR12. The photon radiation field incident on the body depends on the energies and intensities of the photon emissions summarized in Publication 38.

Uncertainties in the intensities of photon emissions are higher for x-rays where the intensities are based, in part, on the fluorescence yield in the electron shells. Differences between theoretical and experimental fluorescent yields increase with increasing electron shell and atomic number. However, the energies of the x-rays from the higher electron shells are low and thus their contribution to organ dose is reduced.

\subsection{Potential errors in external dose coefficients for selected radionuclides}

In occupational and public health applications of external dose coefficients, the largest uncertainties usually arise from differences between the actual distribution of the radionuclide in the environment and the ideal geometries assumed in the derivation of the dose coefficients. For example, differences between the assumed infinite exposure geometries and real, finite exposure geometries often are significant. An example is exposure to contaminated ground surface for which the source region is assumed to be a smooth plane. In reality, external dose rates from sources on the ground surface generally are reduced by shielding due to surface vegetation and irregularities in the terrain. In the present and proposed studies, statements concerning the potential error in an external dose coefficient are not intended to account for the differences between the real exposure geometry and the idealized geometry assumed in FGR12.

If the decay data for a radionuclide are reasonably well established and fully reflected in the dose coefficients in FGR12, the potential errors associated with the dose coefficients are expected to be relatively low for high-energy photons and to increase with decreasing 
photon energy. For some of the radionuclides addressed in FGR12, however, available decay data are not fully reflected in the dose coefficients. For example, the coefficients for ${ }^{252} \mathrm{Cf}$ do not reflect radiations associated with spontaneous fission. Preliminary estimates that include radiations associated with spontaneous fission other than neutrons raise the dose coefficient for exposure to ${ }^{252} \mathrm{Cf}$ on the ground surface by three orders of magnitude. When neutron dose is added (calculations are not yet complete), the dose per unit concentration of ${ }^{252} \mathrm{Cf}$ on the ground surface may approach that for high-energy photon emitters such as ${ }^{60} \mathrm{Co}$.

For the five radionuclides addressed in Section 5, external dose coefficients are given in Table 6.1 for the case of exposure to contaminated ground surface. A qualitative assessment of the potential error associated with each dose coefficient is given in the third column of the table. The potential error is judged to be small for ${ }^{60} \mathrm{Co}$, slightly larger for ${ }^{137} \mathrm{Cs} /{ }^{137 \mathrm{~m}} \mathrm{Ba}$ or ${ }^{106} \mathrm{Ru} /{ }^{106} \mathrm{Rh}$ than for ${ }^{60} \mathrm{Co}$, and large for the low-energy photons associated with ${ }^{90} \mathrm{Sr} /{ }^{90} \mathrm{Y}$. The listed value for ${ }^{252} \mathrm{Cf}$ is orders of magnitude lower than indicated by current information. The potential error is large for ${ }^{252} \mathrm{Cf}$ because the main sources of external dose from ${ }^{252} \mathrm{Cf}$ are photons and neutrons associated with spontaneous fission, and spontaneous fission is not reflected in dose coefficients given in FGR12.

\begin{tabular}{|c|c|c|}
\hline \multicolumn{3}{|c|}{$\begin{array}{l}\text { Table 6.1. Comparative external dose coefficients from FGR12 (EPA, } 1993) \\
\text { for exposure to contaminated ground surface }\left(\mathrm{Sv} \text { per } \mathrm{Bq} \mathrm{s} \mathrm{m}^{-2}\right)\end{array}$} \\
\hline Radionuclide & $\begin{array}{l}\text { Dose coefficient } \\
\text { for red marrow } \\
\text { given in FGR12 } \\
\left(\mathrm{Sv} \text { per Bq } \mathrm{m}^{-2} \text { ) }\right.\end{array}$ & $\begin{array}{l}\text { Potential relative error for the } \\
\text { geometry used in FGR12 }\end{array}$ \\
\hline $\begin{array}{l}{ }^{60} \mathrm{Co} \\
{ }^{137} \mathrm{Cs}+{ }^{137 m} \mathrm{Ba} \\
{ }^{106} \mathrm{Ru}+{ }^{106} \mathrm{Rh} \\
{ }^{90} \mathrm{Sr}+{ }^{90} \mathrm{Y} \\
{ }^{952} \mathrm{Cf}\end{array}$ & $\begin{array}{l}2.3 \mathrm{E}-15 \\
5.7 \mathrm{E}-16 \\
2.1 \mathrm{E}-16 \\
4.8 \mathrm{E}-18 \\
1.9 \mathrm{E}-19\end{array}$ & $\begin{array}{c}\text { Small } \\
\text { Modest } \\
\text { Modest } \\
\text { Large but usually inconsequential } \\
\text { Too low by at least } 3 \text { orders of magnitude }\end{array}$ \\
\hline
\end{tabular}

\subsection{Variation of external dose with body size}

Calculations indicate that the dose to organs of the body from external radiation generally increases with decreasing body size since the shielding from the thickness of overlaying tissue decreases with decreasing body size. Thus, external doses to tissues are expected to be somewhat higher in children than in adults. The dose coefficients in FGR12 are based on an anthropomorphic phantom representing a typical adult male and are expected to underestimate doses received by children to an extent that depends on photon energies. The dependence on body size is more pronounced at low photon energy than at high energy and is also more pronounced for organs located deep in the body than for more shallow organs with less shielding by overlying tissues. Variation of external dose with body size also depends on the nature of the radiation field. 
As an extreme example, consider the dose to the stomach from a broad beam of photons incident on the right side of the body. Under this exposure geometry, the dose to the stomach, most of which is on the left side of the body, is highly sensitive to body size and hence age (Figure 6.1, left panel). The dose to the stomach of a child is estimated to be about 8 times that in the adult. If the photons are incident on the front of the body (Figure 6.1, right panel), the dependence on body size and hence age is reduced and the estimated child-to-adult ratio is about 2.
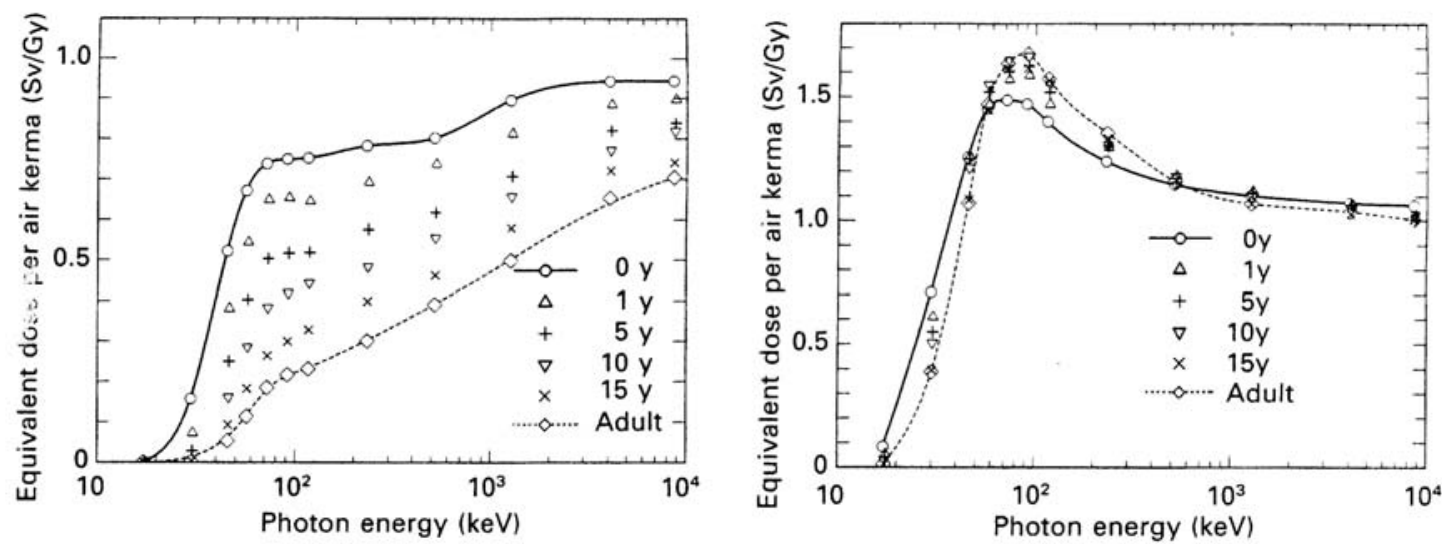

Figure 6.1. Age-specific equivalent dose to the stomach from monoenergetic photons incident on the body (graph on left based on the assumption that the photons are incident on the right side of the body; graph on right is for photons incident on the front of the body) (estimates by Yamaguchi, 1994)

The dependence on body size is further reduced for exposure to a more uniform radiation field. Estimated doses to the liver of the adult male and female are shown in Fig. 6.2 for a monoenergetic photon source uniformly distributed in the air or on the ground surface. In this case, little difference is evident between the male and female at photon energies less than approximately $0.07 \mathrm{MeV}$ for this non-directional exposure. In addition to the nondirectional field, the scatter of photons in air results in a broad spectrum of energies incident on the body. Thus, integration over the energy and angular distribution of the incident radiation field has the effect of reducing the dependence of organ dose on body size.

The effective dose exhibits less dependence on body size than seen in selected organs because it weights the dose to tissues at various depths in the body. This is evident from Figures 6.3, based on the exposure geometries of Figure 6.1, and from Figure 6.4, based on an isotropic radiation field. 


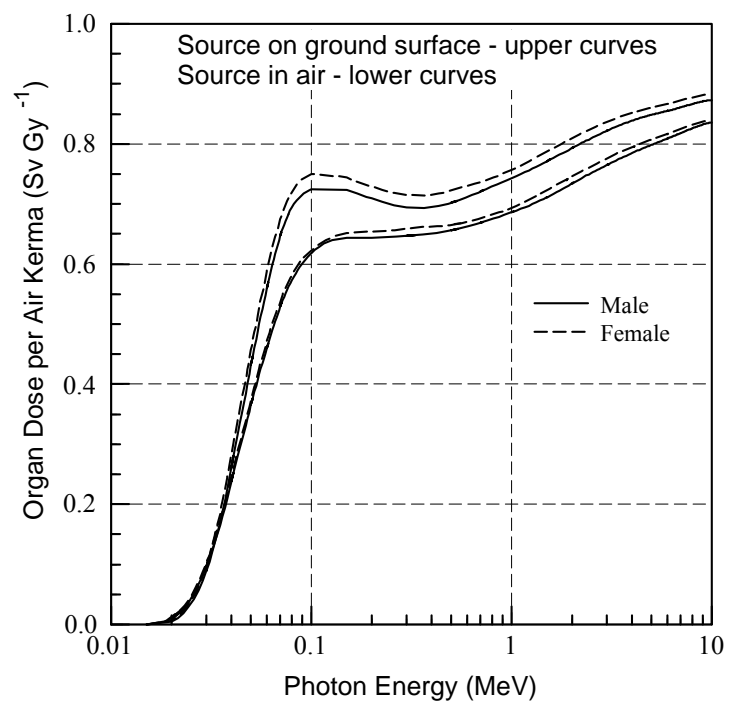

Figure 6.2. The equivalent dose to the liver of an adult male or female for a mononergetic photon source uniformly distributed in the air or on the ground surface (estimates by Zankl et al., 1997)
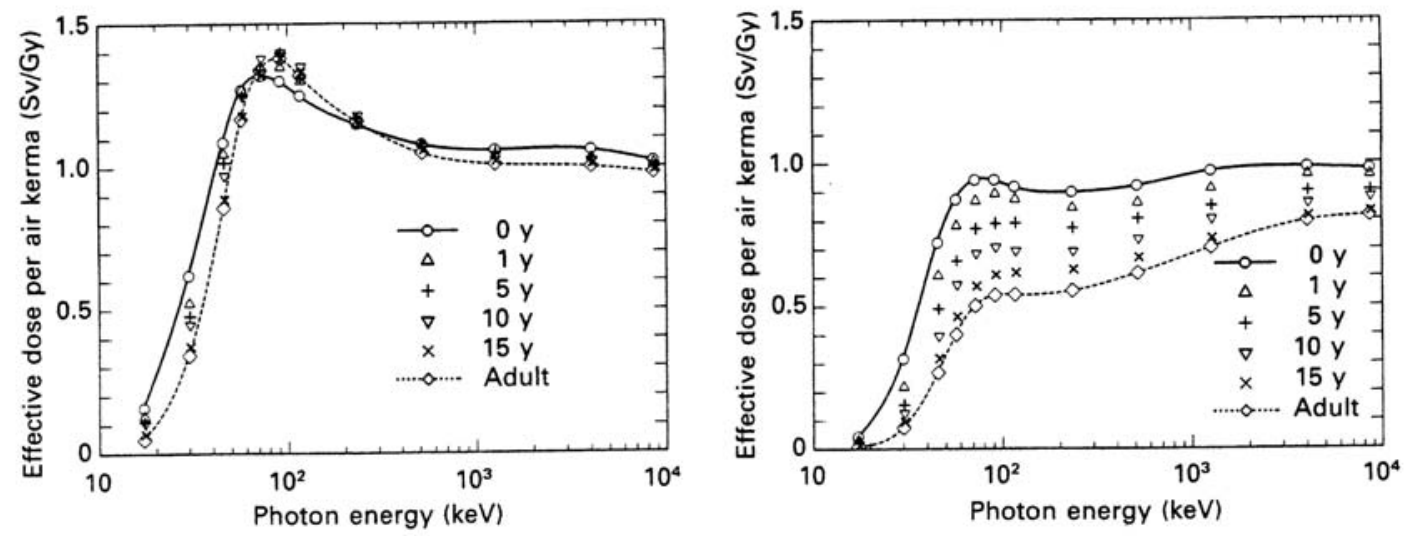

Figure 6.3. Age-specific values of the effective dose per unit air kerma for monoenergetic photons incident on the front of the body (left panel) and on the right side of the body (right panel) (estimates by Yamaguchi, 1994)

The largest uncertainty in application of external dose coefficients for individual organs is associated with assumptions regarding the exposure geometry. In a highly directional radiation field such as that arising from a collimated source, differences in body size lead to substantial uncertainties in the dose to deep organs (Figure 6.1). The dose coefficients indicated in Figures 6.1-6.4 were derived using anatomical models constructed similarly to the model for the adult used in FGR 12. In that model the surface of the body is not represented in a realistic manner, and that may result in greater sensitivity to body size than the voxel models might indicate. As noted above, however, Zankl et al (2002) have 
observed dose differences as large as $100 \%$ among voxel models of adults. Calculations have not been reported for a sufficient number of voxel models of children to quantify the contribution of these anatomical models to uncertainties in the dose coefficients.

Differences between the actual exposure geometry and the idealized geometry used in the computation of dose coefficients remain a source of potential error in assessment of organ dose from external irradiation.

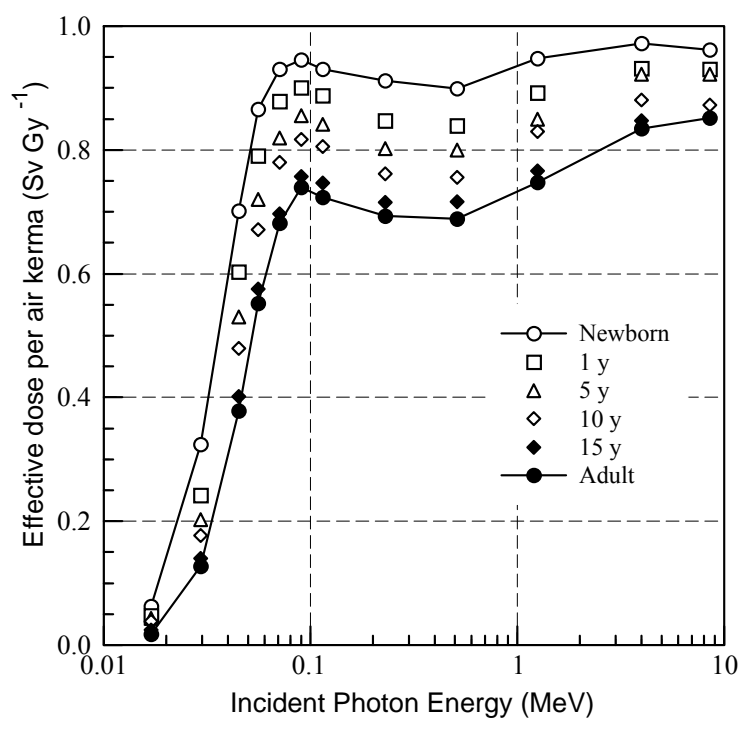

Figure 6.4. Age-specific values of the effective dose per unit air kerma for monoenergetic photons incident on the body in an isotropic geometry (estimates by Yamaguchi 1994) 


\section{DEMONSTRTION OF LEVELS OF POTENTIAL ERROR IN NUCLEAR DECAY DATA USED IN COMPUTATION OF DOSE COEFFICIENTS}

This section discusses the nuclear decay data used to develop current dose coefficients for radionuclides. Although nuclear decay data typically represent a minor source of error in dose estimates, examples are given to illustrate that these data can be an important source of error for some infrequently studied radionuclides.

\subsection{Nuclear decay data tabulations}

Dose coefficients for inhaled and ingested radionuclides published by the ICRP since the issuance of Part 1 of Publication 30 (ICRP, 1979) are based on the nuclear decay data tabulated in ICRP Publication 38 (1983). External dose coefficients tabulated in FGR12 (EPA, 1993) for radionuclides distributed in environment media are also based on ICRP Publication 38.

The data of ICRP Publication 38 were computed by the ORNL code EDISTR (Dillman, 1980) which analyzes information on the nuclear structure assembled as part of the National Nuclear Data Project. The EDISTR code was designed to provide a compilation of the radiations accompanying the nuclear and atomic processes associated with radioactive decay, thus sparing the dosimetrist the tedious task of deriving the energies and intensities of the radiations. The original stimulus for such compilations was the need within the field of nuclear medicine to compute doses from diagnostic radionuclides and radiopharmaceuticals (Dillman, 1969-1970). Following the appearance of ICRP Publication 38, the current MIRD monograph on nuclear decay data was published (Weber et al, 1999). Electronic files of the nuclear decay data tabulated in ICRP Publication 38 have been issued by Eckerman et al. (1994).

The EDISTR code takes basic radioactive decay information as input and calculates intensities and energies of alpha, beta, gamma, internal-conversion electron, x-ray, Auger-electron, and bremsstrahlung radiations emitted as a result of nuclear decay. If spontaneous fission is indicated, the yields of radiations concomitant with spontaneous fission are also computed. Beta spectral shapes are calculated as needed in beta depthdose calculations, e.g., for skin dose. Although the beta spectra were not given in ICRP Publication 38, they were used in the calculations of the skin dose from submersion for noble gas radionuclides and were made available in electronic form by Eckerman et al. (1994). They were also used in calculations done for FGR12 (EPA, 1993). Detailed documentation of the EDISTR code is provided in a report by Dillman (1980).

The mission of the National Nuclear Data Center (NNDC) includes collection, evaluation and dissemination of nuclear physics data for basic nuclear research and application. EDISTR uses data from the Evaluated Nuclear Structure Data File (ENSDF) from NNDC as input. 


\subsection{Selection of radionuclides}

In ICRP Publication 30, the ICRP limited consideration to parent radionuclides with physical half-life $\left(\mathrm{T}_{1 / 2}\right)$ greater than $10 \mathrm{~m}$. ICRP Publication 38 includes decay data for 820 radionuclides, consisting of 764 radionuclides with $T_{1 / 2}$ greater than $10 \mathrm{~m}$ and 56 radionuclides with $\mathrm{T}_{1 / 2}$ less than $10 \mathrm{~m}$ that are either decay chain members subsequent to one or more of those 764 radionuclides, or of interest in medical applications (e.g., $\left.{ }^{13} \mathrm{~N}\right)$. It was also required that the ENSDF for the radionuclide be sufficiently complete to enable EDISTR to indicate a reasonable balance in energy. No substantial effort was undertaken to investigate potential errors in the ENSDF input, although obvious missing data fields were corrected (for example, for identification of chain members). These criteria limited ICRP Publication 38 to 820 radionuclides. The most recent version of NUBASE $^{1}$ (Audi et al, 2003) identifies 3177 radionuclides. The number of radionuclides with $\mathrm{T}_{1 / 2}>10 \mathrm{~m}$ is 1052 , for mass numbers up to 293 .

The decay of a radionuclide generally proceeds promptly to the ground state energy level of the newly formed daughter nucleus. However, nuclear transformation may lead to an energy level in the daughter nucleus, referred to as an isomeric state, from which a transition to the ground state is delayed. The half-lives of isomeric levels observed experimentally can range from $100 \mathrm{~ns}$ up to years. It is necessary to establish criteria as to whether the radiations associated with transitions from the isomeric level are to be included with those of the parent or listed separately. In the development of ICRP Publication 38, an effort was made to identify isomeric states and list the radiations separately if the half-life of the state exceeded $1 \mathrm{~m}$. The rationale was that isomeric states of half-life less than $1 \mathrm{~m}$ would be in secular equilibrium with the parent radionuclide with half-life of $10 \mathrm{~m}$ or more, so that inclusion of the emission with those of the parent seemed justified.

There was at least one inadvertent deviation from this scheme in ICRP Publication 38. Emissions of ${ }^{190 \mathrm{~m}}$ Os were included with those of the parent ${ }^{190 \mathrm{~m}} \operatorname{Ir}\left(\mathrm{T}_{1 / 2}=3.1 \mathrm{~h}\right)$, despite the daughter's half-life of $9.9 \mathrm{~min}$. The emissions of ${ }^{190 \mathrm{~m}}$ Os were separately tabulated; however, it was not identified as a daughter of ${ }^{190 \mathrm{~m}} \mathrm{Ir}$ in the publication. If a user elected to include the ${ }^{190 \mathrm{~m}}$ Os emission with those of ${ }^{190 \mathrm{~m}} \mathrm{Ir}$, then an overestimate of dose would occur because energy from the daughter would be counted twice.

ICRP Publication 38 included a number of isomers for which the ground state had not been clearly identified at the time of publication. For example, data for two isomers of ${ }^{236} \mathrm{~Np}$ were tabulated, one with a half-life of $22.5 \mathrm{~h}$ and the other with a half-life of $1.15 \times 10^{5} \mathrm{y}$. The 22.5 -h isomer is now identified to be an isomeric state and hence designated as ${ }^{236 \mathrm{~m}} \mathrm{~Np}$ (Audi et al. 2003), with the longer lived isomer being the ground state. Similar situations existed for isomers of ${ }^{89} \mathrm{Nb},{ }^{110} \mathrm{In},{ }^{150} \mathrm{Eu},{ }^{186} \mathrm{Ir},{ }^{190 \mathrm{~m} / \mathrm{n}} \mathrm{Ir},{ }^{182} \mathrm{Re}$, ${ }^{120} \mathrm{Sb},{ }^{124 \mathrm{~m} / \mathrm{n}} \mathrm{Sb},{ }^{128} \mathrm{Sb},{ }^{178} \mathrm{Ta}$, and ${ }^{156 \mathrm{~m} / \mathrm{n}} \mathrm{Tb}$.

\footnotetext{
${ }^{1}$ NUBASE is a database which contains values for the basic nuclear properties such as masses, excitation energies of isomers, half-lives, spins and parities, decay models and their intensities.
} 


\subsection{Physical half-life of the radionuclides}

The physical half-life of the radionuclide is often an important parameter in the calculation of dose. For most radionuclides the values of $\mathrm{T}_{1 / 2}$ in ICRP Publication 38 are in good agreement with updated values in NUBASE (Audi et al, 2003) (within 25\%). However, for some long-lived radionuclides, where accurate determination is difficult, the differences can be large. Table 7.1 list radionuclides for which the NUBASE values differ substantially from the values of ICRP Publication 38.

\begin{tabular}{|c|c|c|c|}
\hline \multirow[b]{2}{*}{ Nuclide } & \multirow{2}{*}{\multicolumn{2}{|c|}{ Half-life $T_{1 / 2}$}} & \multirow{2}{*}{$\begin{array}{c}\text { Deviation }^{a} \\
(\%)\end{array}$} \\
\hline & & & \\
\hline In-115 & $5.1 \mathrm{Py}$ & $0.441 \mathrm{Py}$ & $\begin{array}{c}(\%) \\
1100\end{array}$ \\
\hline $\mathrm{Pb}-202$ & 300 ky & $52.5 \mathrm{ky}$ & 470 \\
\hline $\mathrm{Si}-32$ & $450 y$ & $132 \mathrm{y}$ & 240 \\
\hline Tb-157 & $150 y$ & $71 \mathrm{y}$ & 110 \\
\hline $\mathrm{Fe}-60$ & $100 \mathrm{ky}$ & $1.5 \mathrm{My}$ & 93 \\
\hline Se-79 & 65 ky & $295 \mathrm{ky}$ & 78 \\
\hline Es-250 & $2.1 \mathrm{~h}$ & $8.6 \mathrm{~h}$ & 76 \\
\hline $\mathrm{Ag}-108 \mathrm{~m}$ & $127 y$ & $418 \mathrm{y}$ & 70 \\
\hline Sn-126 & $100 \mathrm{ky}$ & 230 ky & 57 \\
\hline $\mathrm{Hg}-194$ & $260 y$ & $440 y$ & 41 \\
\hline Ca-41 & $140 \mathrm{ky}$ & 102 ky & 37 \\
\hline At-218 & $2 \mathrm{~s}$ & $1.5 \mathrm{~s}$ & 33 \\
\hline La-138 & $135 \mathrm{~Gy}$ & $102 G y$ & 32 \\
\hline $\mathrm{Ni}-59$ & 75 ky & $101 \mathrm{ky}$ & 26 \\
\hline Sn-121m & $55 \mathrm{y}$ & $43.9 \mathrm{y}$ & 25 \\
\hline
\end{tabular}

The National Institute of Standards and Technology (NIST) has carried out measurements of $T_{1 / 2}$ for a set of radionuclides of importance in industrial applications. Table 7.2 compares the values observed at NIST with the values in ICRP Publication 38. For most of these radionuclides the values are in reasonable agreement, with discrepancies less that $1 \%$. However, discrepancies beyond reported statistical error are seen for several nuclides as listed in the table, the largest being for ${ }^{207} \mathrm{Bi}$. 


\begin{tabular}{|c|c|c|c|}
\hline \multirow[b]{2}{*}{ Nuclide } & \multicolumn{2}{|c|}{ Half-life $T_{1 / 2}$} & \multirow{2}{*}{$\begin{array}{c}\text { Deviation }^{\text {a }} \\
\text { (\%) }\end{array}$} \\
\hline & ICRP 38 & NIST & \\
\hline $\mathrm{H}-3$ & $4511 d$ & $4500 \pm 8 d$ & $<1$ \\
\hline F-18 & $1.8295 \mathrm{~h}$ & $1.82951 \pm 0.00034 h$ & $<1$ \\
\hline $\mathrm{Na}-22$ & $950.4 \mathrm{~d}$ & $950.97 \pm 0.15 d$ & $<1$ \\
\hline $\mathrm{Na}-24$ & $15 \mathrm{~h}$ & $14.9512 \pm 0.0032 h$ & $<1$ \\
\hline P-32 & $14.29 \mathrm{~d}$ & $14.263 \pm 0.003 d$ & $<1$ \\
\hline Sc-46 & $83.83 \mathrm{~d}$ & $83.831 \pm 0.066 d$ & $<1$ \\
\hline Cr-51 & $27.704 d$ & $27.7010 \pm 0.0012 d$ & $<1$ \\
\hline Mn-54 & $312.5 \mathrm{~d}$ & $312.028 \pm 0.034 d$ & $<1$ \\
\hline Co-57 & $270.9 d$ & $272.11 \pm 0.26 d$ & $<1$ \\
\hline Fe-59 & $44.529 \mathrm{~d}$ & $44.5074 \pm 0.0072 d$ & $<1$ \\
\hline Co-60 & $1925 \mathrm{~d}$ & $1925.20 \pm 0.25 d$ & $<1$ \\
\hline Cu-62 & $9.74 \mathrm{~m}$ & $9.6725 \pm 0.0080 \mathrm{~m}$ & $<1$ \\
\hline Zn-65 & 243.9 & $244.164 \pm 0.099 d$ & $<1$ \\
\hline Ga-67 & $3.261 \mathrm{~d}$ & $3.26154 \pm 0.00054 d$ & $<1$ \\
\hline Se-75 & $119.8 \mathrm{~d}$ & $119.809 \pm 0.066 d$ & $<1$ \\
\hline Kr-85 & $3915 \mathrm{~d}$ & $3935.7 \pm 1.2 \mathrm{~d}$ & $<1$ \\
\hline Sr-85 & $64.84 \mathrm{~d}$ & $64.8530 \pm 0.0081 d$ & $<1$ \\
\hline Y-88 & $106.64 \mathrm{~d}$ & $106.626 \pm 0.044 d$ & $<1$ \\
\hline Mo-99 & $66.0 \mathrm{~h}$ & $65.9239 \pm 0.0058 h$ & $<1$ \\
\hline Tc-99m & $6.02 \mathrm{~h}$ & $6.0123 \pm 0.0032 h$ & $<1$ \\
\hline Ru-103 & $39.28 \mathrm{~d}$ & $39.310 \pm 0.044 d$ & $<1$ \\
\hline Cd-109 & $464 d$ & $463.26 \pm 0.63 d$ & $<1$ \\
\hline $\mathrm{Ag}-110 \mathrm{~m}$ & $249.9 d$ & $249.950 \pm 0.024 d$ & $<1$ \\
\hline $\ln -111$ & $2.83 \mathrm{~d}$ & $2.80477 \pm 0.00053 d$ & $<1$ \\
\hline Sn-113 & $115.1 d$ & $115.079 \pm 0.080 d$ & $<1$ \\
\hline Sn-117m & $13.61 \mathrm{~d}$ & $14.00 \pm 0.05 d$ & 2.8 \\
\hline $\mathrm{I}-123$ & $13.2 \mathrm{~h}$ & $13.2235 \pm 0.0019 h$ & $<1$ \\
\hline Sb-125 & $1010 d$ & $1007.56 \pm 0.10 \mathrm{~d}$ & $<1$ \\
\hline |-125 & $60.14 \mathrm{~d}$ & $59.49 \pm 0.13 d$ & 1.1 \\
\hline Xe-127 & $36.41 \mathrm{~d}$ & $36.3446 \pm 0.0028 d$ & $<1$ \\
\hline |-131 & $8.04 d$ & $8.0197 \pm 0.0022 d$ & $<1$ \\
\hline$X e-131 m$ & $11.9 \mathrm{~d}$ & $11.934 \pm 0.021 d$ & $<1$ \\
\hline Ba-133 & $3923 d$ & $3854.7 \pm 2.8 d$ & 1.8 \\
\hline Xe-133 & $5.245 \mathrm{~d}$ & $5.24747 \pm 0.00045 d$ & $<1$ \\
\hline Cs-134 & $753.1 \mathrm{~d}$ & $753.88 \pm 0.15 d$ & $<1$ \\
\hline Cs-137 & $10960 \mathrm{~d}$ & $11018.3 \pm 0.5 d$ & $<1$ \\
\hline Ce-139 & $137.66 \mathrm{~d}$ & $137.734 \pm 0.091 d$ & $<1$ \\
\hline Ba-140 & $12.74 \mathrm{~d}$ & $12.7527 \pm 0.0023 d$ & $<1$ \\
\hline La-140 & $40.272 \mathrm{~h}$ & $40.293 \pm 0.012 h$ & $<1$ \\
\hline Ce-141 & $32.501 \mathrm{~d}$ & $32.510 \pm 0.024 d$ & $<1$ \\
\hline Ce-144 & $284.3 \mathrm{~d}$ & $284.534 \pm 0.032 d$ & $<1$ \\
\hline Eu-152 & $4869 d$ & $4947.2 \pm 1.1 \mathrm{~d}$ & 1.6 \\
\hline Gd-153 & $242 d$ & $239.472 \pm 0.069 d$ & 1.1 \\
\hline Sm-153 & $46.7 \mathrm{~h}$ & $46.2853 \pm 0.0014 h$ & $<1$ \\
\hline Eu-154 & $3210 d$ & $3145.2 \pm 1.1 \mathrm{~d}$ & 2.1 \\
\hline Eu-155 & $1810 \mathrm{~d}$ & $1739.06 \pm 0.45 d$ & 4.1 \\
\hline Но-166 & $26.80 \mathrm{~h}$ & $26.794 \pm 0.023 h$ & $<1$ \\
\hline Yb-169 & $32.01 \mathrm{~d}$ & $32.0147 \pm 0.0093 d$ & $<1$ \\
\hline Lu-177 & $6.71 \mathrm{~d}$ & $6.64 \pm 0.01 d$ & $<1$ \\
\hline W-181 & $121.2 \mathrm{~d}$ & $121.095 \pm 0.064 d$ & $<1$ \\
\hline
\end{tabular}




\begin{tabular}{lccc}
\hline \multicolumn{4}{c}{ Table 7.2 (cont'd) } \\
$\mathrm{Re}-186$ & $90.64 \mathrm{~h}$ & $89.248 \pm 0.069 \mathrm{~h}$ & 1.6 \\
$\mathrm{Re}-188$ & $16.98 \mathrm{~h}$ & $17.001 \pm 0.022 \mathrm{~h}$ & $<1$ \\
$\mathrm{Ir}-192$ & $74.02 \mathrm{~d}$ & $73.810 \pm 0.019 \mathrm{~d}$ & $<1$ \\
$\mathrm{Au}-195$ & $183 \mathrm{~d}$ & $186.098 \pm 0.047 \mathrm{~d}$ & 1.7 \\
$\mathrm{Au}-198$ & $2.696 \mathrm{~d}$ & $2.69517 \pm 0.00021 \mathrm{~d}$ & $<1$ \\
$\mathrm{Tl}-201$ & $3.044 \mathrm{~d}$ & $3.0456 \pm 0.0015 \mathrm{~d}$ & $<1$ \\
$\mathrm{Tl}-202$ & $12.23 \mathrm{~d}$ & $12.466 \pm 0.081 \mathrm{~d}$ & $<1$ \\
$\mathrm{Hg}-203$ & $46.60 \mathrm{~d}$ & $46.619 \pm 0.027 \mathrm{~d}$ & $<1$ \\
$\mathrm{~Pb}-203$ & $52.05 \mathrm{~h}$ & $51.923 \pm 0.037 \mathrm{~h}$ & $<1$ \\
$\mathrm{Bi}-207$ & $13880 \mathrm{~d}$ & $11523 \pm 15 \mathrm{~d}$ & 20 \\
$\mathrm{Th}-228$ & $698.7 \mathrm{~d}$ & $698.60 \pm 0.36 \mathrm{~d}$ & $<1$ \\
\hline${ }^{\mathrm{a}} 100$ (absolute value of difference)/ NIST \\
\hline \multicolumn{4}{l}{} \\
\hline
\end{tabular}

\subsection{Total energy of emitted radiations}

Uncertainty in the total energy emitted by the various types of radiations accompanying radioactive decay translates directly to uncertainty in the computed tissue dose. Revised information on the decay process (i.e., a change in the decay scheme) can have a substantial effect on the partitioning of the available energy. For example ${ }^{80} \mathrm{Sr}$ decays by electron capture. In ICRP Publication 38, 100\% of the transitions were depicted as leading to the ground state of ${ }^{80} \mathrm{Rb}$ (Figure 7.1, left). Recent information (Endo et al., 2005) indicates that transitions occur to four excited states in ${ }^{80} \mathrm{Rb}$ as well as the ground state and that decay by positron emission is energetically possible (Figure 7.1, right). The identified gamma emissions from the excited states and associated radiations substantially increase the estimate of total energy emissions (Table 7.3).

Other examples of potentially important updates in nuclear decay data are changes in the branching fraction for alpha decay in ${ }^{202} \mathrm{~Pb}$, and the revision of the electron capture to positron ratio for ${ }^{135} \mathrm{Ce}$. The main consideration regarding the total energy emission is the quality of the decay scheme information.

\begin{tabular}{lcc}
\hline \multicolumn{3}{c}{ Table 7.3. Energy of radiations emitted by Sr-80 } \\
\hline \multicolumn{3}{c}{ Emitted Energy $\left(\mathrm{MeV} \text { nt }^{-1}\right)^{\mathrm{a}}$} \\
\hline Radiation & ICRP 38 & JAERI - 1347 \\
\hline$\gamma$-rays & - & 0.3278 \\
X-rays & 0.0080 & 0.0073 \\
$Y^{ \pm}$ & - & 0.1020 \\
$\beta+$ & - & 0.0357 \\
IC electrons & - & 0.0013 \\
Auger & 0.0055 & 0.0049 \\
Total & 0.0135 & 0.4789 \\
\hline${ }^{a}$ nt = nuclear transformation \\
\hline
\end{tabular}




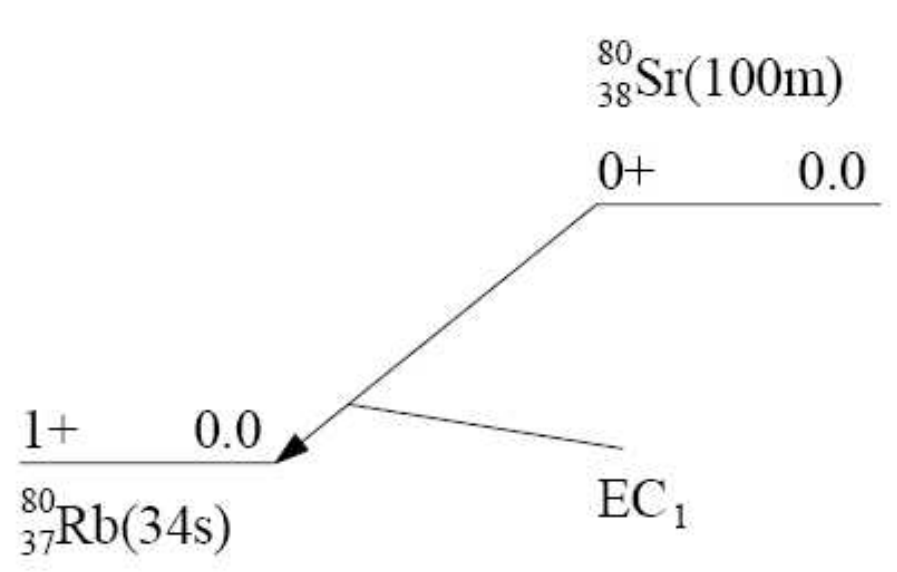

ICRP Publication 38 decay scheme for ${ }^{80} \mathrm{Sr}$

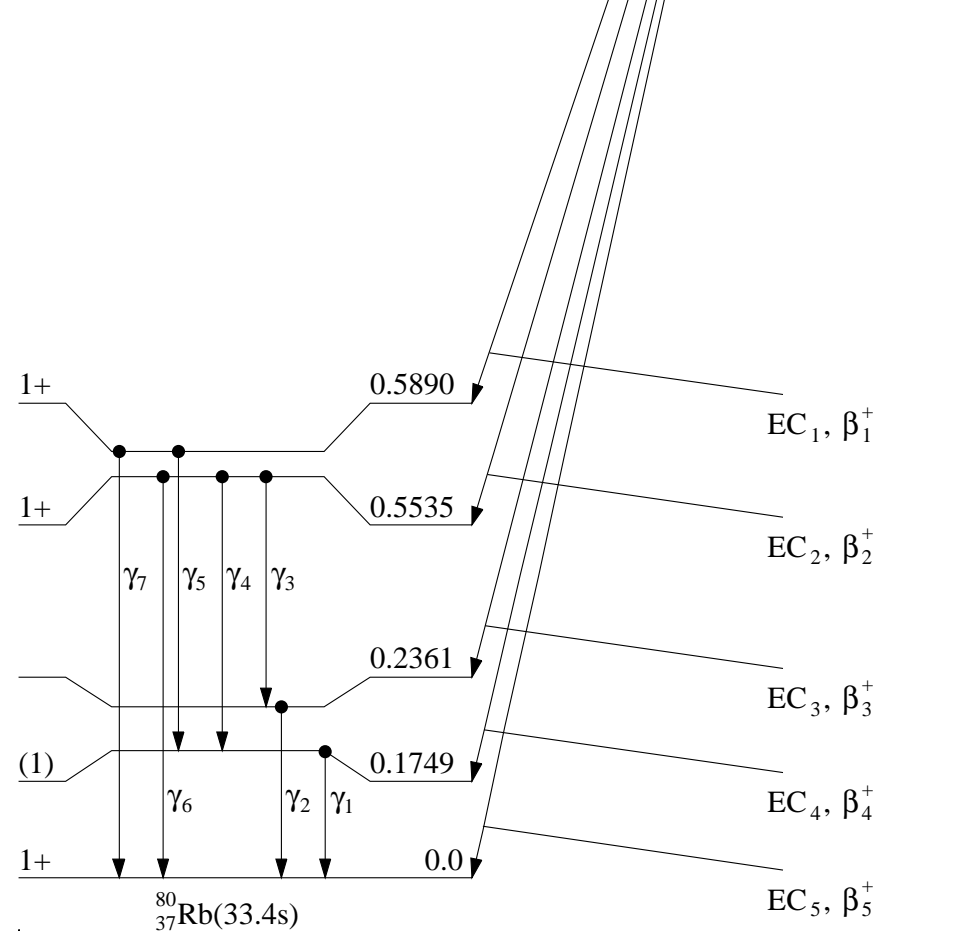

Updated decay scheme for ${ }^{80} \mathrm{Sr}$ (Endo et al., 2005)

Figure 7.1. Decay scheme for ${ }^{80} \mathrm{Sr}$ given in ICRP Publication 38 (1983) compared with JAERI 1347 (Endo et al., 2005) 


\subsection{Radiations from spontaneous fission}

Spontaneous fission is a decay mode of some heavy nuclei where the nucleus breaks into two intermediate-mass fragments and several neutrons. It has been observed only for nuclei with atomic numbers greater than 230 . The large amount of energy involved in this process is distributed among fission fragments and prompt neutrons and gamma emissions. In addition, delayed beta and gamma emissions occur from the newly formed fission fragments. EDISTR adopted the methods of Dillman and Jones (1975) for calculating the energies and intensities of spontaneous fission emissions.

The energy of the fission fragments is an important issue for internally deposited radionuclides, because $\sim 80 \%$ of the available energy is associated with the fragments. It has been shown that total kinetic energy of the fragments as described by a simple Coulomb repulsion model agrees within about 5\% with the experimental data.

In EDISTR the Maxwellian distribution was used to calculate the average energy of prompt neutrons. The widely used radiation transport code MCNP represents the neutron spectrum shape by the Watt spectrum as do some other Monte Carlo transport codes. The total energy of the prompt neutron spectrum is proportional to the number of prompt neutrons emitted per fission. These data have been updated to some extent since the preparation of ICRP Publication 38, but changes are generally minor. For example, Dillman and Jones (1975) suggested values of 2.07, 2.24, 3.14 and 3.73 for ${ }^{238} \mathrm{U},{ }^{242} \mathrm{Pu}$,

${ }^{248} \mathrm{Cm}$, and ${ }^{252} \mathrm{Cf}$, compared with recent estimates of 2.01, 2.15, 3.11 and 3.765 respectively (Wilson et al, 2002).

Shortly after fissioning and the prompt emission of neutrons, the remaining energy of the fission fragments is released as prompt gamma rays. EDISTR assigned a gamma-ray energy value of $7.64 \mathrm{MeV}$ per fission for all spontaneous fission nuclides. A set of equations developed by Valentine (2001) yield estimates that are somewhat different from the EDISTR value but are in close agreement with updated experimental data. These equations indicate a total energy of prompt gamma ray emissions per fission of 6.61, 6.54, 6.78 and 6.94 for ${ }^{233} \mathrm{U},{ }^{235} \mathrm{U},{ }^{239} \mathrm{Pu}$, and ${ }^{252} \mathrm{Cf}$, respectively, compared with the value of 7.64 assumed in EDISTR.

After the fission fragments reach ground state or isomeric states, further energy is emitted by beta decay with accompanying gamma emissions. Exact treatment of these emissions would require consideration of the yield of fission fragments and tracking the change in the composition of these products over time. For an internal source, this would also entail consideration of the biokinetics of each individual member. For example, spontaneous fission of ${ }^{252} \mathrm{Cf}$ yields over 700 radionuclides of about 50 elements. Dillman and Jones (1975) developed equations for predicting the energy of delayed beta and gamma emissions based on the number of beta decays to reach a stable nuclide. The empirical equations are based on experimental data on spontaneous fission of ${ }^{236} \mathrm{U}$. The predictions of these equations are reasonably consistent with experimental data. 


\subsection{Conclusions}

For most common radionuclides the nuclear decay processes are sufficiently well understood that uncertainties in the energies and intensities of the emission accompanying nuclear decay are minor. Considerable uncertainty often exists in the physical half-life of long-lived radionuclides, which can be important in issues regarding expected environmental behavior as in the case of ${ }^{79} \mathrm{Se}$. Uncertainty in the electron capture to positron ratio can result in substantial uncertainty in the total energy emission for this decay mode. Increased use of accelerators in production of isotopes for medical use and for neutron studies (e.g., spallation neutron sources) can result in production of radionuclides for which experimental data are weak or missing. 


\section{RECOMMENDATIONS FOR A COMPREHENSIVE STUDY}

This section describes a general approach for assessing and reducing potential errors in dose estimates for radionuclides. The approach consists of a series of steps to be followed for each exposure case, where an exposure case is defined as a given combination of radionuclide, exposure mode, and (when pertinent) specified form of the radionuclide and level of knowledge of that form. A list of 50 radionuclides is proposed for consideration, based on their importance as indicated by past experience or the potential for elevated exposures in the future. Estimates of the time, funds, and other resources required to carry out a detailed assessment for this set of radionuclides are provided.

\subsection{Definitions and practical bounds for a comprehensive study}

\subsubsection{Definitions of uncertainty and variability}

Publications addressing uncertainties in biokinetic and dosimetric quantities for radionuclides generally do not clearly define what is meant by "uncertainty". In many cases, it appears from the discussions and analytical methods that the reference may be to an unknown individual. In the present pilot study and for purposes of the proposed comprehensive study, uncertainty refers to knowledge concerning a central value (usually, an arithmetic or geometric mean) for the population of interest. The uncertainty in the central value of a quantity in the population should not be confused with the "variability" of that feature in the population. Variability refers to quantitative differences between different members of a population under similar conditions (interindividual variability) or within an individual under different conditions (intra-individual variability).

\subsubsection{Potential error compared with uncertainty and variability}

The discussions and analyses in this report have emphasized the importance of evaluating the potential error associated with a dose estimator rather than simply focusing on uncertainty and variability, which are contributing factors to the potential error but often are not the only contributing factors. The potential error in an application of a dose coefficient stems in part from limitations in the information needed to construct the biokinetic and dosimetric models used to develop the coefficient, but the additional problem arises in many cases that the models do not fully or accurately reflect available information. The latter problem may reflect expansion of the database after the construction of the models but can also arise from poor modeling practices such as selective or uncritical use of data or inadequate review of the database. One of the early steps in assessing the uncertainties in dose estimates should be to attempt to differentiate between fixable errors in the underlying models, stemming from incomplete or improper use of the database, and actual uncertainties in model estimates arising from inadequate data. Where feasible, models should be modified or replaced to adequately reflect the full, critically reviewed database before an uncertainty assessment is initiated. 


\subsubsection{The need to avoid new dosimetric frontiers}

For the comprehensive study to be completed in a reasonable length of time at a reasonable cost, attention should be limited to the following topics:

o potential errors in equivalent dose to tissues per unit intake or per unit external exposure to radionuclides;

0 the sensitivity of the effective dose to potential errors in equivalent doses to tissues.

It is not feasible to address issues such as the appropriateness of the ICRP's definition of tissue dose or effective dose as measures of radiogenic risk.

\subsubsection{Exposure modes to be addressed}

For a given radionuclide, the analysis should focus on potential errors for:

0 ingestion of a unit activity of the radionuclide; where necessary, different characterizations should be given for different forms of the radionuclide or different levels of knowledge of the form, e.g. ${ }^{137} \mathrm{Cs}$ known to be in highly absorbable form, and ${ }^{137} \mathrm{Cs}$ ingested in an unknown form

o inhalation of a unit activity of different general forms (e.g., known to be highly soluble, known to be moderately soluble, or form unknown)

0 any other mode of uptake to blood that has been shown to be important for the radionuclide of interest, e.g., absorption of ${ }^{3} \mathrm{H}$ or ${ }^{131} \mathrm{I}$ through the skin or intake of ${ }^{239} \mathrm{Pu}$ or ${ }^{241} \mathrm{Am}$ through wounds

0 external exposure to the radionuclide on the ground surface, based on the idealized exposure geometry (infinite extent) considered in FGR12

o external exposure due to submersion in air, based on the idealized exposure geometry (infinite extent) considered in FGR12

\subsubsection{Radionuclides to be addressed}

ICRP documents provide dose coefficients for ingestion or inhalation of over 800 radionuclides. For the case of inhalation, different dose coefficients are provided for different absorption types and sometimes for different particle sizes. Federal Guidance Report No. 12 tabulates thousands of dose coefficients covering a number of different external exposure scenarios.

It is not feasible to provide careful uncertainty assessments for all of these thousands of cases. The full uncertainty study should focus on radionuclides for which elevated exposures to human beings seem most likely on the basis of past experience and consideration of potential uses of radionuclides in the near future. For example, the full study should address radionuclides frequently encountered in occupational settings, released in potentially significant quantities due to reactor operations or accidents (Table 8.1), or mentioned frequently in the NRC's database of reportable events, or radionuclides that may be dispersed due to terrorist events involving radiological dispersal devices or nuclear explosions. 


\begin{tabular}{|c|c|c|c|c|c|}
\hline \multirow{2}{*}{$\begin{array}{l}\text { Exposed } \\
\text { persons }\end{array}$} & \multirow{2}{*}{$\begin{array}{l}\text { Time after } \\
\text { accident (d) }\end{array}$} & \multicolumn{2}{|c|}{ Internal exposure ${ }^{a, b}$} & \multicolumn{2}{|c|}{ External exposure $^{a}$} \\
\hline & & Inhalation & Ingestion & Gamma & Beta \\
\hline \multirow{3}{*}{$\begin{array}{l}\text { Plant staff, } \\
\text { emergency } \\
\text { workers, } \\
\text { mitigators }\end{array}$} & $1-10$ & $\begin{array}{l}{ }^{131} \mathrm{I},{ }^{133} \mathrm{I}, \\
{ }^{132} \mathrm{Te}, \mathrm{TUE}\end{array}$ & - & $\begin{array}{l}{ }^{132} \mathrm{Te},{ }^{131} \mathrm{I}, \\
\text { noble gases }\end{array}$ & $\begin{array}{l}{ }^{106} \mathrm{Ru},{ }^{144} \mathrm{Ce}, \\
{ }^{132} \mathrm{Te}\end{array}$ \\
\hline & $11-100$ & TUE, ${ }^{144} \mathrm{Ce}$ & - & $\begin{array}{l}{ }^{95} \mathrm{Zr},{ }^{106} \mathrm{Ru}, \\
{ }^{134} \mathrm{Cs},{ }^{137} \mathrm{Cs}\end{array}$ & ${ }^{106} \mathrm{Ru},{ }^{144} \mathrm{Ce}$ \\
\hline & $>100$ & TUE, ${ }^{144} \mathrm{Ce}$ & - & $\begin{array}{l}{ }^{106} \mathrm{Ru},{ }^{134} \mathrm{Cs}, \\
{ }^{137} \mathrm{Cs}\end{array}$ & $\begin{array}{l}{ }^{106} \mathrm{Ru},{ }^{144} \mathrm{Ce}, \\
{ }^{90} \mathrm{Sr}\end{array}$ \\
\hline $\begin{array}{l}\text { Evacuated } \\
\text { population }\end{array}$ & $1-11$ & $\begin{array}{l}{ }^{131} \mathrm{I},{ }^{133} \mathrm{I}, \\
{ }^{132} \mathrm{Te}, \mathrm{TUE}\end{array}$ & 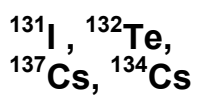 & $\begin{array}{l}{ }^{132} \mathrm{Te},{ }^{131} \mathrm{I}, \\
\text { noble gases }\end{array}$ & $\begin{array}{l}{ }^{106} \mathrm{Ru},{ }^{144} \mathrm{Ce}, \\
{ }^{132} \mathrm{Te}\end{array}$ \\
\hline \multirow[t]{2}{*}{$\begin{array}{l}\text { Rest of } \\
\text { population }\end{array}$} & $<100$ & ${ }^{131}$ I, TUE & $\begin{array}{l}{ }^{131} \mathrm{I},{ }^{137} \mathrm{Cs}, \\
{ }^{134} \mathrm{Cs},{ }^{89} \mathrm{Sr}\end{array}$ & $\begin{array}{l}{ }^{132} \mathrm{Te},{ }^{131} \mathrm{I},{ }^{134} \mathrm{Cs} \\
{ }^{137} \mathrm{Cs}\end{array}$ & ${ }^{106} \mathrm{Ru},{ }^{132} \mathrm{Te}$ \\
\hline & $>100$ & ${ }^{{ }_{144} \mathrm{Ce}}{ }^{{ }^{106} \mathrm{Ru},}$ & $\begin{array}{l}{ }^{137} \mathrm{Cs},{ }^{134} \mathrm{Cs}, \\
{ }^{90} \mathrm{Sr}\end{array}$ & $\begin{array}{l}{ }^{134} \mathrm{Cs},{ }^{137} \mathrm{Cs}, \\
{ }^{106} \mathrm{Ru}\end{array}$ & - \\
\hline
\end{tabular}

${ }^{a}$ Short-lived daughters not listed; e.g., ${ }^{137} \mathrm{Cs}$ as a source of gamma radiation refers to its short-lived daughter ${ }^{137 \mathrm{~m}} \mathrm{Ba}$.

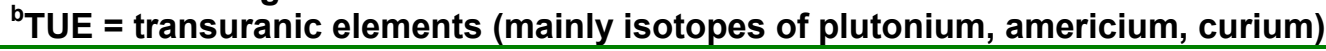

Based on these considerations, we have developed a list of 50 radionuclides to be addressed in a comprehensive study. These have been divided into Group I, Group II, and Group III, to be addressed in the same order. It is expected that the full list will be modified somewhat and the suggested order of treatment of radionuclides may be changed to address specific needs of funding agencies.

Group I: ${ }^{32} \mathrm{P},{ }^{60} \mathrm{Co},{ }^{63} \mathrm{Ni},{ }^{85} \mathrm{Sr},{ }^{89} \mathrm{Sr},{ }^{90} \mathrm{Sr},{ }^{134} \mathrm{Cs},{ }^{137} \mathrm{Cs},{ }^{125} \mathrm{I},{ }^{131} \mathrm{I},{ }^{192} \mathrm{Ir},{ }^{238} \mathrm{U},{ }^{238} \mathrm{Pu},{ }^{241} \mathrm{Am}$, ${ }^{252} \mathrm{Cf}$

Group II: ${ }^{3} \mathrm{H},{ }^{14} \mathrm{C},{ }^{35} \mathrm{~S},{ }^{91} \mathrm{Y},{ }^{99 \mathrm{~m}} \mathrm{Tc},{ }^{103} \mathrm{Pd},{ }^{103} \mathrm{Ru},{ }^{106} \mathrm{Ru},{ }^{109} \mathrm{Cd},{ }^{147} \mathrm{Pm},{ }^{210} \mathrm{Po},{ }^{226} \mathrm{Ra},{ }^{227} \mathrm{Ac}$, ${ }^{239} \mathrm{Pu},{ }^{240} \mathrm{Pu},{ }^{241} \mathrm{Pu},{ }^{242} \mathrm{Pu}$

Group III: ${ }^{55} \mathrm{Fe},{ }^{59} \mathrm{Fe},{ }^{86} \mathrm{Rb},{ }^{95} \mathrm{Zr},{ }^{95} \mathrm{Nb},{ }^{99} \mathrm{Mo},{ }^{110 \mathrm{~m}} \mathrm{Ag},{ }^{125} \mathrm{Sb},{ }^{132} \mathrm{Te},{ }^{140} \mathrm{Ba},{ }^{140} \mathrm{La},{ }^{141} \mathrm{Ce}$, ${ }^{144} \mathrm{Ce},{ }^{197} \mathrm{Hg},{ }^{203} \mathrm{Hg},{ }^{207} \mathrm{Bi},{ }^{237} \mathrm{~Np},{ }^{239} \mathrm{~Np},{ }^{242} \mathrm{Cm}$

Group I includes several radionuclides that appear frequently in the NRC's history of reportable events $\left({ }^{137} \mathrm{Cs}>{ }^{241} \mathrm{Am}>{ }^{192} \mathrm{Ir}>{ }^{131} \mathrm{I}>{ }^{238} \mathrm{U}>{ }^{63} \mathrm{Ni}>{ }^{60} \mathrm{Co}>{ }^{125} \mathrm{I}>{ }^{90} \mathrm{Sr}>{ }^{32} \mathrm{P}\right)$, some other important isotopes of the same elements (e.g., ${ }^{85} \mathrm{Sr},{ }^{89} \mathrm{Sr},{ }^{134} \mathrm{Cs}$ ), and some radionuclides of concern with regard to potential use in radiological dispersal devices $\left({ }^{238} \mathrm{Pu}\right.$ and ${ }^{252} \mathrm{Cf}$, in addition to some radionuclides already included due to the frequency of occurrence in the NRC database $-{ }^{60} \mathrm{Co},{ }^{90} \mathrm{Sr},{ }^{137} \mathrm{Cs},{ }^{192} \mathrm{Ir}$, and ${ }^{241} \mathrm{Am}$ ). In this list, ${ }^{238} \mathrm{U}$ represents natural uranium isotopes; the biokinetics, dosimetry, uncertainty, and variability are expected to be virtually the same for ${ }^{238} U$ as for ${ }^{234} U$ or ${ }^{235} U$. Five of the 
radionuclides in Group I represent about $75 \%$ of the leaking source events $\left({ }^{63} \mathrm{Ni}>{ }^{137} \mathrm{Cs}>\right.$ $\left.{ }^{241} \mathrm{Am}>{ }^{125} \mathrm{I}>{ }^{90} \mathrm{Sr}\right)$, and seven represent about $85 \%$ of the reported medical events $\left({ }^{131} \mathrm{I}>\right.$ ${ }^{192} \mathrm{Ir}>{ }^{137} \mathrm{Cs}={ }^{60} \mathrm{Co}>{ }^{90} \mathrm{Sr}>{ }^{125} \mathrm{I}>{ }^{32} \mathrm{P}$ ) listed in the NRC database. Seven of the radionuclides, ${ }^{60} \mathrm{Co},{ }^{90} \mathrm{Sr},{ }^{137} \mathrm{Cs},{ }^{192} \mathrm{Ir},{ }^{241} \mathrm{Am},{ }^{252} \mathrm{Cf}$, and ${ }^{238} \mathrm{Pu}$, are the radionuclides of greatest concern as components of dirty bombs according to some assessments because of their portability, dispersibility, and widespread use in commercial sources, as well as their potential hazards as internal or external emitters.

Group II includes several other radionuclides that appear frequently in the NRC database of reportable events: ${ }^{3} \mathrm{H}>{ }^{99 m} \mathrm{Tc}>{ }^{210} \mathrm{Po}>{ }^{35} \mathrm{~S}>{ }^{109} \mathrm{Cd}>{ }^{14} \mathrm{C}>{ }^{147} \mathrm{Pm}>{ }^{226} \mathrm{Ra}>{ }^{103} \mathrm{Pd}$. Other radionuclides are included in Group II because they are potentially important sources of dose following reactor accidents and are sometimes mentioned in the literature with regard to occupational exposure or exposure to fallout (e.g., ${ }^{106} \mathrm{Ru}$ ). Actinium-227 is included mainly because fixable errors in its published dose coefficients have important implications with regard to transport regulations for radioactive material containing unknown radionuclides.

Group III includes several other radionuclides that appear a number of times in the NRC database of reportable events. This group also includes a few remaining radionuclides such as ${ }^{95} \mathrm{Zr},{ }^{95} \mathrm{Nb},{ }^{132} \mathrm{Te}$, and ${ }^{144} \mathrm{Ce}$, that have been addressed frequently in the literature, mainly because of their potential importance in occupational settings, releases following reactor accidents, or fallout.

\subsection{Proposed methods of assessment of potential error, uncertainty, and variability}

\subsubsection{Inhalation or ingestion of radionuclides}

Step 1: Specify the case (radionuclide, intake mode, and form of the radionuclide or level of knowledge concerning the form)

As discussed in Section 2, a number of papers and reports address "the uncertainty in a dose coefficient" for an internal emitter, but this is not a well defined concept. The level of confidence that can be placed in a dose coefficient for ingestion or inhalation may vary considerably from one application to another, depending on the form of the radionuclide and the level of knowledge concerning that form. While one could assign either a minimal uncertainty or a worst-case uncertainty to an ingestion or inhalation dose coefficient, either approach could be misleading in many applications.

A workable solution is to develop different uncertainty statements for different forms of radionuclides or different levels of knowledge of the form. For example, an uncertainty statement could be provided for an ingestion dose coefficient for ${ }^{137} \mathrm{Cs}$ under the assumption that the radionuclide is incorporated in food or ingested in soluble inorganic form, and an alternate uncertainty statement could be derived for arbitrary use of the dose coefficient, i.e., in the absence of knowledge of the form of ${ }^{137} \mathrm{Cs}$ ingested. 
The number of different cases to be considered for inhalation or ingestion of a radionuclide will vary from one radionuclide to another. For inhalation of some radionuclides, it may be necessary to address several different forms or levels of knowledge concerning the form.

\section{Step 2: Critically review the biokinetic and dosimetric database for the radionuclide and its physiological analogues.}

Usually, this is most efficiently done by element rather than by radionuclide. For some radionuclides it may be necessary to address specific compounds; e.g., for ${ }^{14} \mathrm{C}$, it is useful to address $\mathrm{CO}_{2}$ separately from most other carbon compounds.

\section{Step 3: Evaluate current dose models for a radionuclide against the critically reviewed data.}

If current models are supported by the critically reviewed data, go to Step 4 . If inconsistencies between the models and database are judged to contribute substantially to potential errors in dose estimates, modify or replace the models insofar as feasible within the time constraints of the project.

Several examples were given in Sections 3 and 5 in which comparison of critically reviewed data with current ICRP models revealed major problems in the models. For example, in Section 3.3.7 it is pointed out that a review of the database for inhaled mercury vapor revealed that the ICRP's recommended half-time for mercury vapor deposited in the lungs $(1.7 \mathrm{~d})$ was based on misinterpretation of the original data, and revised parameter values were derived. Reassessment of the data indicates that the ICRP's current model for inhaled mercury vapor may overestimate lung dose by an order of magnitude for inhalation of most mercury isotopes. Of course, some uncertainty remains in the revised model for mercury vapor, but the potential error in applying the model has been reduced.

\section{Step 4: Assess the sensitivity of dose estimates to uncertainties in model structures, parameter values, and assumptions of the underlying models}

The "underlying models" refer to current ICRP models if no improvements were indicated in Step 3 and to the modified models if improvements were made.

The method commonly used to examine sensitivity of dose estimates to uncertainties in underlying data is parameter uncertainty analysis. This method was discussed in Sections 3 and 5 in connection with various case studies. The method can be instructive, but it is not well suited to most biokinetic models used in radiation protection. These models generally are not process models, or not entirely process models, and many or all of their parameter values may not be observable quantities. Moreover, an analysis of the sensitivity of dose estimates to gaps in knowledge must take into account that the model structure may be incomplete, incorrect, or misleading, and that model assumptions (e.g., regarding the biokinetics of radioactive progeny produced in vivo) that are not generally 
treated in parameter uncertainty analyses may be important sources of uncertainty in dose estimates.

An approach to analyzing sensitivity of dose to gaps in information that has stronger logical support is comparison of predictions of different but equally plausible models. This approach was illustrated in Sections 3-5.

The approach of comparing equally plausible models was used by the authors and coworkers at EPA to assess uncertainty in each of the thousands of risk coefficients given in FGR13 (the full uncertainty analysis is not yet published). For each radionuclide and exposure mode, up to 54 different models were compared. A difficulty with that analysis was that the number of cases to be considered was so large that it was difficult to ensure that the different models were indeed equally plausible and that the investigators had not overlooked modeling approaches that would produce a wider range of plausible dose or risk estimates. It is for such reasons that we recommend that attention be restricted to about 50 radionuclides and a small number of exposure modes (see Section 8.2.5) in the full project proposed here.

The specific quantities for which uncertainties are to be quantified should include estimates of the 50-y committed dose equivalent. Also, it would be useful to quantify uncertainties in urinary excretion rates, because urinary data are commonly used in dose reconstructions.

Finally, it may be prudent to avoid very specific quantitative statements of uncertainty and instead summarize uncertainty results in terms of relatively broad uncertainty categories. These categories could represent a range of knowledge, e.g., well known, moderately well known, poorly known, and very poorly known. Such an approach was used in FGR13 (see Table 2.4 of that report). Of course, documentation of the analysis should include quantitative results on which the assignment of exposure cases to uncertainty categories is based. Such quantitative results are illustrated in Table 8.2 for ingestion or inhalation of ${ }^{137} \mathrm{Cs}$, either in soluble form or in an unknown form.

\section{Step 5. Characterize variability}

Variability of dose from intake of a radionuclide should be characterized only insofar as relevant observations are available for the radionuclide of interest or for its established physiological analogues or governing physiological processes. For example, sufficient measurements on human subjects are available to characterize variation of biokinetics and dose from ingestion of soluble ${ }^{137} \mathrm{Cs}$ or ${ }^{90} \mathrm{Sr}$ with age, gender, and certain states of health. Although direct observations on the variability in the biokinetics of ${ }^{3} \mathrm{H}$ taken in as tritiated water are sparse, the behavior of ${ }^{3} \mathrm{H}$ is known to be tied closely to water replacement in the body, and variability with age, gender, and certain states of health in water replacement is reasonably well understood. On the other hand, no particularly useful information is available on differences with age or gender in the biokinetics of inhaled ${ }^{106} \mathrm{Ru}$, and there are no known close physiological analogues of ruthenium. 
Therefore, variability in the biokinetics or dose per unit intake of ${ }^{106} \mathrm{Ru}$ might be characterized simply as "unknown".

\begin{tabular}{|c|c|c|c|c|c|c|}
\hline $\begin{array}{l}\text { Intake } \\
\text { mode }\end{array}$ & Form & Tissue & $\begin{array}{c}\text { Best } \\
\text { estimate } \\
\left(\mathrm{Sv} \mathrm{Bq}^{-1}\right)\end{array}$ & $\begin{array}{l}\text { Lower } \\
\text { bound } \\
\left(\mathrm{Sv} \mathrm{Bq}^{-1}\right)\end{array}$ & $\begin{array}{l}\text { Upper } \\
\text { bound } \\
\left(\mathrm{Sv} \mathrm{Bq}^{-1}\right)\end{array}$ & $\begin{array}{l}\text { Ratio of } \\
\text { upper to } \\
\text { lower } \\
\text { bound }\end{array}$ \\
\hline Ingestion & $\begin{array}{l}\text { Soluble } \\
\text { inorganic or } \\
\text { incorporated } \\
\text { in food }\end{array}$ & $\begin{array}{l}\text { Lung } \\
\text { Colon } \\
\text { Red Marrow } \\
\text { Effective dose }\end{array}$ & $\begin{array}{l}8.0 \mathrm{E}-9 \\
1.8 \mathrm{E}-8 \\
1.5 \mathrm{E}-8 \\
1.1 \mathrm{E}-8\end{array}$ & $\begin{array}{l}\text { 6.0E-9 } \\
9.0 \mathrm{E}-9 \\
8.0 \mathrm{E}-9 \\
8.0 \mathrm{E}-9\end{array}$ & $\begin{array}{l}1.8 \mathrm{E}-8 \\
2.7 \mathrm{E}-8 \\
2.4 \mathrm{E}-8 \\
2.4 \mathrm{E}-8\end{array}$ & $\begin{array}{l}3.0 \\
3.0 \\
3.0 \\
3.0\end{array}$ \\
\hline Ingestion & Unknown form & $\begin{array}{l}\text { Lung } \\
\text { Colon } \\
\text { Red Marrow } \\
\text { Effective dose }\end{array}$ & $\begin{array}{l}8.0 \mathrm{E}-9 \\
1.8 \mathrm{E}-8 \\
1.5 \mathrm{E}-8 \\
1.1 \mathrm{E}-8\end{array}$ & $\begin{array}{l}0.6 \mathrm{E}-9 \\
9.0 \mathrm{E}-9 \\
0.8 \mathrm{E}-9 \\
1.5 \mathrm{E}-9\end{array}$ & $\begin{array}{l}1.8 \mathrm{E}-8 \\
3.6 \mathrm{E}-8 \\
2.4 \mathrm{E}-8 \\
2.4 \mathrm{E}-8\end{array}$ & $\begin{array}{l}30 \\
4.0 \\
30 \\
16\end{array}$ \\
\hline Inhalation & Soluble & $\begin{array}{l}\text { Lung } \\
\text { Colon } \\
\text { Red Marrow } \\
\text { Effective dose }\end{array}$ & $\begin{array}{l}2.7 \mathrm{E}-9 \\
6.2 \mathrm{E}-9 \\
5.2 \mathrm{E}-9 \\
3.9 \mathrm{E}-9\end{array}$ & $\begin{array}{l}2.0 \mathrm{E}-9 \\
2.0 \mathrm{E}-9 \\
2.0 \mathrm{E}-9 \\
2.0 \mathrm{E}-9\end{array}$ & $\begin{array}{l}1.4 \mathrm{E}-8 \\
7.0 \mathrm{E}-9 \\
7.0 \mathrm{E}-9 \\
7.0 \mathrm{E}-9\end{array}$ & $\begin{array}{l}7.0 \\
3.5 \\
3.5 \\
3.5\end{array}$ \\
\hline Inhalation & Unknown form & $\begin{array}{l}\text { Lung } \\
\text { Colon } \\
\text { Red Marrow } \\
\text { Effective dose }\end{array}$ & $\begin{array}{l}2.7 \mathrm{E}-9 \\
6.2 \mathrm{E}-9 \\
5.2 \mathrm{E}-9 \\
3.9 \mathrm{E}-9\end{array}$ & $\begin{array}{l}2.0 \mathrm{E}-9 \\
2.0 \mathrm{E}-9 \\
2.0 \mathrm{E}-9 \\
2.0 \mathrm{E}-9\end{array}$ & $\begin{array}{l}4.0 \mathrm{E}-7 \\
8.0 \mathrm{E}-9 \\
1.2 \mathrm{E}-8 \\
5.0 \mathrm{E}-8\end{array}$ & $\begin{array}{l}200 \\
4.0 \\
6.0 \\
25\end{array}$ \\
\hline
\end{tabular}

As illustrated in Table 8.3, statistical analysis of reported biokinetic data for some frequently studied radionuclides will allow estimates of variation of certain biokinetic or dosimetric properties between subjects of the same age and gender. Such information should be documented as part of this project as an indication of the status of knowledge of dose per unit intake for individuals or subgroups of the population.

\subsubsection{External dose}

Characterization of the uncertainty in an external dose coefficient for a radionuclide, assuming an idealized exposure geometry as defined in FGR12, is essentially a propagation of errors associated with three sources of uncertainty: the nuclear decay data for the radionuclide; the radiation field incident upon the body for the specified exposure geometry; and the dose rate to specific tissues due to a body surface source, taking body size into account.

The uncertainties in nuclear decay data are negligible for most radionuclides; some exceptions are discussed in Section 7. The error in the estimated radiation field incident upon the body depends on the energy of the radiations, and error levels have been 
determined by comparison of FGR12 estimates with actual measurements and with other theoretical models. Errors in estimated dose rates to tissues due to a body surface source arise in large part from the simplified representation of the human body as a mathematical phantom and generally increase with decreasing energy. Comparisons with voxel phantoms provide an indication of energy-specific levels of error associated with these dose rates.

\begin{tabular}{|c|c|c|c|c|c|}
\hline Quantity & $\begin{array}{c}\text { No. of } \\
\text { subjects }\end{array}$ & $\begin{array}{c}\text { Geometric } \\
\text { mean } \\
(\%)\end{array}$ & $\begin{array}{c}\text { Geometric } \\
\text { S.D. }\end{array}$ & $\begin{array}{c}\text { Observed } \\
\text { maximum } \\
(\%)\end{array}$ & $\begin{array}{c}\text { Estimated } \\
\text { 98th } \\
\text { percentile }\end{array}$ \\
\hline Retention at $5 \mathrm{~d}$ (injection) & 6 & $65 \%$ & 1.1 & $77 \%$ & $83 \%$ \\
\hline Retention, at $50 \mathrm{~d}$ (injection) & 7 & $25 \%$ & 1.4 & $38 \%$ & $48 \%$ \\
\hline Retention at $200 \mathrm{~d}$ (injection) & 5 & $4.3 \%$ & 1.4 & $5.7 \%$ & $8.3 \%$ \\
\hline Retention at $6 \mathrm{~d}$ (ingestion) & 8 & $14 \%$ & 1.6 & $28 \%$ & $39 \%$ \\
\hline Retention at $20 \mathrm{~d}$ (ingestion) & 9 & $9.3 \%$ & 1.7 & $22 \%$ & $28 \%$ \\
\hline Retention at $50 \mathrm{~d}$ (ingestion) & 8 & $5.0 \%$ & 1.6 & $10 \%$ & $13 \%$ \\
\hline Urinary Sr on day 4 (injection) & 5 & $1.7 \%$ & 1.2 & $2.3 \%$ & $2.5 \%$ \\
\hline Urinary Sr on day 4 (ingestion) & 9 & $0.41 \%$ & 1.6 & $0.57 \%$ & $1.2 \%$ \\
\hline Fecal Sr on day 4 (injection) & 4 & $0.52 \%$ & 1.4 & $0.68 \%$ & $1.1 \%$ \\
\hline
\end{tabular}

\subsection{Estimates of resources required for a comprehensive project}

It is expected that the project as described in this section could be completed in three years at a level of effort of 1.4 man-years per year. Group I, Group II, and Group III radionuclides would be addressed during the first, second, and third years of the project, respectively. Approximately $\$ 18 \mathrm{~K}$ would be needed for library costs (ordering papers and reports) and equipment. The equipment would include two relatively high-speed desktop computers and software such as the latest version of Crystal Ball, to be used mainly for quality assurance of the software developed by the investigators. 


\section{REFERENCES}

Aden, J.; Scott, B. R. (2003). Modeling variability and uncertainty associated with inhaled weapons-grade $\mathrm{PuO}_{2}$. Health Phys.84:726-736.

Anderson, J.; Tomlinson, R. W. S.; Ramsay, I. D.; Clarke, M. B.; Osborn, S. B. (1970). Radiocalcium uptake in the bones of the foot. Br. J. Radiol. 43:168-172.

Apostoaei, A. I. Absorption of strontium from the gastrointestinal tract into plasma in healthy adult humans. Health Phys. 83:56-65; 2002.

Apostoaei, A. I.; Lewis, C. J.; Hammonds, J. H.; Hoffman, F. O. (1998). Uncertainties in doses from ingestion of Cs-137, Sr-90, Co-60, Ru-106, and I-131. Health Phys. 74:S14S15.

Apostoaei, A. I.; Miller, L. F. (2004). Uncertainties in dose coefficients from ingestion of ${ }^{131} \mathrm{I},{ }^{137} \mathrm{Cs}$, and ${ }^{90}$ Sr. Health Phys. 86:460-482.

Arnold, J. S.; Stover, B. J.; Van Dilla, M. B. (1955). Failure of Y-90 to escape from skeletally-fixed Sr-90. Soc. Exp. Biol. Med. 90:260-263.

Atherton, D. R.; Lloyd, R. D. (1972). The distribution and retention of ${ }^{249} \mathrm{Cf}$ in beagle soft tissue. Health Phys. 22:675-677.

Audi, G.; Bersillon, O.; Blachot, J.; Wapstra A. H. (2003). The NUBASE evaluation of nuclear and decay properties. Nucl. Phys. A729:3-128.

Bailey, M. R. (1994). The new ICRP model for the respiratory tract. Radiat. Prot. Dosim. 53:107-114.

Bailey, M. R.; Ansoborlo, E.; Guilmette, R. A.; Paquet, F. (2003). Practical application of the ICRP human respiratory tract model. Radiat. Prot. Dosim. 105:71-76.

Balonov, M. I. (1993). Overview of doses to the Soviet population from the Chernobyl accident and the protective actions applied. (S. E. Merwin and M. I. Balonov, eds.) The Chernobyl papers. Vol. I. Doses to the Soviet population and early health effects studies, pp. 47-70.

Beleznay, E.; Osvay, M. (1994). Long-term clearance of accidentally inhaled 60Co aerosols in humans. Health Phys. 66:392-399.

Birchall, A.; James, A. C. (1994). Uncertainty analysis of the effective dose per unit exposure from radon progeny and implications for ICRP risk-weighting factors. Radiat. Prot. Dosim. 133-140; 1994. 
Bishop, M.; Harrison, G. E.; Raymond, W. H. A.; Sutton, A.; Rundo, J. (1960). Excretion and retention of radioactive strontium in normal man following a single intravenous injection. Int. J. Radiat. Biol. 2:125-142.

Bligh, P. H.; Taylor, D. M. (1963). Comparative studies of the metabolism of strontium and barium in the rat. Biochem. J. 87:612-618.

Bogen, K. T.; Conrado, C. L.; Robison, W. L. (1997). Uncertainty and variability in updated estimates of potential dose and risk at a U.S. nuclear test site--Bikini Atoll. Health Phys. 73:115-126.

Bolch, W. E.; Farfan, E. B.; Huh, C. H.; Huston, T. E.; Bolch, W. E. (2001). Influences of parameter uncertainties within the ICRP-66 respiratory tract model: Particle deposition. Health Phys. 81:378-394.

Bolch, W. E.; Huston, T. E.; Farfan, E. B.; Vernetson, W. G.; Bolch, W. E. (2003). Influences of parameter uncertainties within the ICRP-66 respiratory tract model: Particle clearance. Health Phys. 84:421-435.

Borle, A. B. (1981). Control, modulation, and regulation of cell calcium. Rev. Physiol. Biochem. Pharmacol. 9:13-153.

Bruenger, F. W.; Atherton, D. R.; Stevens, W. (1972). Intracellular distribution of ${ }^{249} \mathrm{Cf}$ in canine liver. Health Phys. 22:685-689.

Burykina, L. N. (1962). The metabolism of radioactive ruthenium in the organism of experimental animals. In: The toxicology of radioactive substances, Vol. 1 (A. A. Letavet and E. B. Kurlyandskaya, eds.) Oxford: Pergamon Press; 60-76.

Campbell, J. E.; Robajdek, E. S.; Anthony, D. S. The metabolism of ${ }^{227}$ Ac and its daughters ${ }^{227}$ Th and ${ }^{223}$ Ra by rats. Radiat. Res. 4:294-302; 1956.

Carr, T. E. F.; Harrison, G. E.; Nolan, J. (1973). The long-term excretion and retention of an intravenous dose of Ca-45 in two healthy men. Calcif. Tissue Res. 12:217-226.

Christensen, J. M. (1995). Human exposure to toxic metals: factors influencing interpretation of biomonitoring results. Sci. Total Environ. 166:89-135.

Comar, C. L.; Wasserman, R. H.; Ullberg, S.; Andrews, G. A. (1957). Strontium metabolism and strontium-calcium discrimination in man. Soc. Exp. Biol. Med. 95:386391.

Dillman, L. T. (1969). Radionuclide decay schemes and nuclear parameters for use in radiation-dose estimation. MIRD Pamphlet No. 4, J Nucl Med 10:Suppl No 2. 
Dillman, L. T. (1970). Radionuclide decay schemes and nuclear parameters for use in radiation-dose estimation, part 2. MIRD Pamphlet No. 6, J Nucl Med 11:Suppl No 4.

Dillman, L. T. (1980). EDISTR- A computer program to obtain a nuclear decay data bases for radiation dosimetry. ORNL/TM6689.

Dillman, L. T.; Jones, T. D. (1975). Internal dosimetry of spontaneously fissioning nuclides, Health Phys. 29:111-123.

Domanski, T.; Liniecki, J.; Witkowska, D. (1969). Kinetics of calcium, strontium, barium, and radium in rats. In: Delayed Effects of Bone-Seeking Radionuclides; ed. C. W. Mays, Univ. Utah Press, Salt Lake City pp. 79-.

Domanski, T.; Witkowska, D.; Garlicka, I. (1980). Influence of age on the discrimination of barium in comparison with strontium during their incorporation into compact bone. Acta Physiol. Pol. 31:289-296.

Durbin, P. W. (1960). Metabolic characteristics within a chemical family. Health Phys. 2:225-238; 1960.

Durbin, P. W. (1973). Metabolism and Biological Effects of the Transuranium Elements. In: Uranium, Plutonium and Transplutonic Elements. Eds. H. C. Hodge, J. N. Stannard and J. B. Hursh. Handbook of Experimental Pharmacology. (Berlin, Springer-Verlag) pp.739-896.

Eckerman, K. F.; Westfall, R. J.; Ryman, J. C.; Cristy, M. (1994). Availability of nuclear decay data in electronic form, including beta spectra not previously published. Health Phys. 67:338-345.

Eisenman, G. (1963). The influence of $\mathrm{Na}, \mathrm{K}, \mathrm{Li}, \mathrm{Rb}$, and $\mathrm{Cs}$ on cellular potentials and related phenomena. Bol. Inst. Estud. Med. Biol. (Mex.); 21:155-183.

Ellsasser, J. C.; Farnham, J. E.; Marshall, J. H. (1969). Comparative kinetics and autoradiography of Ca-45 and Ba-133 in ten-year-old beagle dogs. J. Bone Jt. Surg. 51A:1397-1412.

Endo, A.; Yamaguchi, Y.; Eckerman, K. F. (2005). Nuclear decay data for dosimetry Calculation, JAERI 1347.

EPA (1988). FGR11. Limiting values of radionuclide intake and air concentration and dose conversion factors for inhalation, submersion, and ingestion, Federal Guidance Report No. 11, EPA-520/1-88-020 (Oak Ridge National Laboratory, Oak Ridge, TN; U. S. Environmental Protection Agency, Washington, DC). 
EPA (1993). FGR12. External exposure to radionuclides in air, water, and soil, Federal Guidance Report No. 12, EPA-402-R-93-081 (Oak Ridge National Laboratory, Oak Ridge, TN; U. S. Environmental Protection Agency, Washington, DC).

EPA (1999). FGR13. Cancer risk coefficients for environmental exposure to radionuclides. Federal Guidance Report No. 13, EPA 402-R-99-001. Prepared by Eckerman, K. F.; Leggett, R. W.; Nelson, C. B.; Puskin, J. S.; Richardson, A. C. B.

Farfan, E. B.; Huston, T. E.; Bolch, W. E.; Vernetson, W. G.; Bolch, W. E. (2003). Influences of parameter uncertainties within the ICRP-66 respiratory tract model: Regional tissue doses for ${ }^{239} \mathrm{PuO}_{2}$ and ${ }^{238} \mathrm{UO}_{2} /{ }^{238} \mathrm{U}_{3} \mathrm{O}_{8}$. Health Phys. 84:436-450.

Furchner, J. E.; Richmond, C. R.; Drake, G. A. (1971). Comparative metabolism of radionuclides in mammals. VII. Retention of $106 \mathrm{Ru}$ in the mouse, rat, monkey and dog. Health Phys. 21:355-365.

Graham, B. W.; Ziemer, P. L.; Landolt, R. R.; Shaw, S. M. (1978). The metabolism of

${ }^{252} \mathrm{CfCl}_{3}$ in the rat and the effect of in vivo DTPA chelation therapy. Health Phys. 34:635641.

Harrison, G. E.; Carr, T. E. F.; Sutton, A. (1967). Distribution of radioactive calcium, strontium, barium and radium following intravenous injection into a healthy man. Int. J. Radiat. Biol. 13:235-247.

Harrison, J. D., Metivier, H., Leggett, R. W., Nosske, D., Paquet, F., Phipps, A. and Taylor, D. (2001). Reliability of the ICRP's dose coefficients for members of the public. II. Uncertainties in the absorption of ingested radionuclides and the effect on dose estimates. Radiat. Prot. Dosim. 95, 295-308.

Hart, H. E.; Spencer, H. (1976). Vascular and extravascular calcium interchange in man determined with radioactive calcium. Radiat. Res. 67:149-161; 1976.

Healy, J. W. (1957). Estimation of plutonium lung burden by urine analysis. Am. Ind. Hyg. Assoc. Quarterly 18:261-266.

Heaney, R. P. (1964). Evaluation and interpretation of calcium-kinetic data in man. Clin. Orthop. Rel. Res. 31:153-183.

Heard, M. J.; Chamberlain, A. C. (1984). Uptake of Pb by human skeleton and comparative metabolism of $\mathrm{Pb}$ and alkaline earth elements. Health Phys. 47:857-865.

Henrichs, K.; Werner, E.; Schmitt, A. (1984). Re-evaluation of radiation exposure due to administration of radioactive calcium-47. In: IRPA, 6th Congress, Berlin, May 7-12, $1984 ; 458-461$. 
Huston, T. E.; Farfan, E. B.; Bolch, W. Emmett; Bolch, Wesley. E. (2003). Influences of parameter uncertainties within the ICRP-66 respiratory tract model: a parameter sensitivity analysis. Health Phys. 85:553-66.

ICRP (1973). International Commission on Radiological Protection, Publication 20, Alkaline Earth Metabolism in Adult Man. Oxford: Pergamon Press.

ICRP (1975). International Commission on Radiological Protection, Report of the Task Group on Reference Man, ICRP Publication 23. Oxford: Pergamon Press.

ICRP (1979). Limits for intakes of radionuclides by workers. ICRP Publication 30, Part 1. Oxford: Pergamon Press.

ICRP (1980). International Commission on Radiological Protection. Limits for intakes by workers. ICRP Publication 30, Part 2. Oxford: Pergamon Press.

ICRP (1983). International Commission on Radiological Protection. Radionuclide transformations energy and intensity of emissions. ICRP Publication 38. Oxford: Pergamon Press.

ICRP (1989). International Commission on Radiological Protection. Age-dependent doses to members of the public from intake of radionuclides, Part 1, ICRP Pub. 56. Oxford: Pergamon Press.

ICRP (1993). International Commission on Radiological Protection. Age-dependent doses to members of the public from intake of radionuclides: Part 2. ICRP Publication 67. Oxford: Pergamon Press.

ICRP (1994a). International Commission on Radiological Protection. Human respiratory tract model for radiological protection, ICRP Publication 66. Oxford: Pergamon Press.

ICRP (1994b). International Commission on Radiological Protection. Dose coefficients for intakes of radionuclides by workers, ICRP Publication 68. Oxford: Pergamon Press.

ICRP (1995a). International Commission on Radiological Protection. Age-dependent doses to members of the public from intake of radionuclides, Part 3, ICRP Pub. 69. Oxford: Pergamon Press.

ICRP (1995b). International Commission on Radiological Protection. Age-dependent doses to members of the public from intake of radionuclides, Part 4, ICRP Pub. 71. Oxford: Pergamon Press.

ICRP (1997). International Commission on Radiological Protection. Individual monitoring for internal exposure of workers. Replacement of ICRP Publication 54. ICRP Publication 78. Oxford: Pergamon Press. 
ICRP (1998). International Commission on Radiological Protection (ICRP). Radiation dose to patients from radiopharmaceuticals. Addendum to ICRP 53. ICRP Publication 80. Oxford: Pergamon Press.

ICRP (2002a). International Commission on Radiological Protection. Supporting guidance 3. Guide for the practical application of the ICRP Human Respiratory Tract Model. Oxford: Pergamon Press.

ICRP (2002b). International Commission on Radiological Protection. Basic anatomical and physiological data for use in radiological protection: Reference values. ICRP Pub. 89. Oxford: Pergamon Press.

Inaba, J., Nishimura, Y., Kimura, K.-I., Ichikawa, R. (1982). Whole-body retention and tissue distribution of ${ }^{60} \mathrm{Co}$ in rats after oral administration of freshwater fish contaminated with ${ }^{60} \mathrm{Co}$. Health Phys. 43:247-250.

Jansen, H. M; Knollema, S.; van der Duin, L. V.; Willemsen, A. T.; Wiersma, A.; Franssen, E. J.; Russel, F. G.; Korf, J.; Paans, A. M. (1996). Pharmacokinetics and dosimetry of cobalt-55 and cobalt-57. J Nucl Med. 37:2082-2086.

Kernan, R. P. (1972). Studies of cesium uptake by rat soleus and vastus lateralis muscles in vivo and of its efflux rate relative to potassium in vitro. Pflug. Arch.; 333:95-110.

Khokhryakov, V. F.; Menshikh, Z. S.; Suslova, K. G.; Kudryavtseva, T. I.; Tokarskaya, Z. B.; Romanov, S. A. (1994). Plutonium excretion model for the healthy man. Radiat. Prot. Dosim. 53:235-239.

Khokhryakov, V. F.; Suslova, K. G.; Filipy, R. E.; Alldredge, J. R.; Aladova, E. E.; Glover, S. E.; Vostrotin, V. V. (2000). Metabolism and dosimetry of actinide elements in occupationally exposed personnel of Russia and the United States: A summary progress report. Health Phys. 79:63-71.

Khokhryakov, V. F.; Suslova, K. G.; Vostrotin, V. V.; Romanov, S. A.; Eckerman, K. F.; Krahenbuhl, M. P.; Miller, S. C. Adaptation of the ICRP Publication 66 respiratory tract model to data on plutonium biokinetics for Mayak workers. Health Phys. 88:125-132.

King, W. C. (1980). Use of AERIN code for determining internal doses of transuranic isotopes. Paper prepared for presentation at the Health Physics Society Meeting at Seattle, Washington, July 21-25, 1980; UCRL-84520, CONF-800731--2:1-15.

Korsunskii, V. N.; Tarasov, N. F.; Naumenko, A. Z. (1981). Clinical evaluation of Ba$133 \mathrm{~m}$ as an osteotropic agent. ORNL/TR-86/30 (7 pgs); Translated from the Russian UDC 616.71-006-073.916, Meditsinskaya Radiologiya No. 10, pp. 45-48.

Kosako T, Nakamura T, Ohkubo T. (1981) [An urgent radioactivity detection on ${ }^{252} \mathrm{Cf}$ contamination accident] Radioisotopes 30:352-9. [Article in Japanese] 
Kreyling, W. G., Ferron, G. A., Haider, B. (1986). Metabolic fate of inhaled Co aerosols in beagle dogs. Health Phys. 51:773-795; 1986.

Kshirsagar, S. G.; Lloyd, E.; Vaughan, J. (1966). Discrimination between strontium and calcium in bone and the transfer from blood to bone in the rabbit. Br. J. Radiol. 39:131140 .

Layton, D. W. (1993). Metabolically consistent breathing rates for use in dose assessments, Health Phys. 64:23-36.

Lebedev, O. V., Yakovlev, V. A. (1993). The correlation between 137Cs half-time and age, body mass, and height in individuals contaminated from the Chernobyl accident. (S. E. Merwin and M. I. Balonov, eds.) The Chernobyl papers. Vol. I. Doses to the Soviet population and early health effects studies, pp. 219-243.

Leggett, R. W. (1986). Predicting the retention of cesium in individuals. Health Phys. 50:747-759.

Leggett, R. W. (1992). A generic age-specific biokinetic model for calcium-like elements, Radiat. Prot. Dosim. 41:183-198.

Leggett, R. W. (2001). Consistent biokinetic models for the actinide elements. Rad. Prot. Env. 24:616-622.

Leggett, R. W.; Bouville, A.; Eckerman, K. F. (1998). Reliability of the ICRP's systemic biokinetic models. Radiat. Prot. Dosim. 79:335-342.

Leggett, R. W.; Eckerman, K. F.; Boice, J. D. (2005). A respiratory model for uranium aluminide based on occupational data. J. Radiol. Prot. 25:1-12.

Leggett, R. W.; Munro, N. B.; Eckerman, K. F. (2001). Proposed revision of the ICRP model for inhaled mercury vapor. Health Phys. 81:450-455.

Leggett, R. W.; Williams, L. R.; Melo, D. R.; Lipsztein, J. L. (2003). A physiologically based biokinetic model for cesium in the human body. Sci. Total Environ. 317:235-255.

Letourneau, E. G.; Jack, G. C.; McCullough, R. S.; Hollins, J. G. (1972). The metabolism of cobalt by the normal human male: Whole body retention and radiation dosimetry. Health Phys. 22:451-459.

Likhtarev, I. A.; Dobroskok, I. A.; Ilyin, L. A.; Krasnoschekova, G. P.; Likhtareva, T. M.; Smirnov, B. I.; Sobolev, E. P.; Shamov, V. P.; Shapiro, E. L. (1975). A study of certain characteristics of strontium metabolism in a homogeneous group of human subjects. Health Phys. 28:49-60. 
Liniecki, J. (1971). Kinetics of calcium, strontium, barium and radium in rabbits. Health Phys. 21:367-376.

Lloyd, R. D.; Mays, C. W.; McFarland, S. S.; Zundel, W. S.; Tyler, F. H. (1973). Metabolism of Rb-83 and Cs-137 in persons with muscle disease. Radiat. Res. 54:463-478.

Lloyd, E. (1961). The relative distributions of radioactive yttrium and strontium and the secondary deposition of ${ }^{90} \mathrm{Y}$ built up from ${ }^{90} \mathrm{Sr}$; Int. J. Rad. Biol. 3:475-492.

Lloyd, R. D.; Mays, C. W.; Atherton, D. R. (1976). Distribution of injected Ra-226 and Sr-90 in the beagle skeleton. Health Phys. 30:183-189; 1976.

Lloyd, R. D.; Mays, C. W.; McFarland, S. S.; Atherton, D.R.; Williams, J. L. Californium studies in beagles. Radiat. Res. 65:462-473; 1976.

Lloyd, R. D.; Mays, C. W.; Taylor, G. N.; Williams, J. L. Californium excretion and retention by beagles injected with ${ }^{249} \mathrm{Cf}$ or ${ }^{252} \mathrm{Cf}$. Health Phys. 22:667-673; 1972.

Lloyd, R. D.; Zundel, W. S.; Mays, C. W.; Wagner, W. W.; Pendelton, R. C. (1966). Enhanced Cs-137 elimination in pregnant and dystrophic humans. Radiat. Res. 27:548.

Malluche, H. H.; Werner, E.; Ritz, E. (1978). Intestinal absorption of calcium and wholebody calcium retention in incipient and advanced renal failure. Miner. Electrolyte Metab. 1:263-270.

Marsh, J. W.; Birchall, A.; Butterweck, G.; Dorrian, M. D.; Huet, C.; Ortega, X.; Reineking, A.; Tymen, G.; Schuler, Ch.; Vargas, A.; Vezzu, G.; Wendt, J. Uncertainty analysis of the weighted equivalent lung dose per unit exposure to radon progeny in the home. Radiat Prot Dosimetry 102:229-248; 2002.

Martin, R. C.; Knauer, J. B.; Balo, P. A. (2000). Production, distribution and applications of californium-252 neutron sources. Appl. Radiat. Isot. 53:785-792.

Mays, C. W.; Lloyd, R. D.; Christensen, W. R.; Atherton, D. R.; Pitchford, G. S. (1963). Radium metabolism in a man. Radiat. Res. 19:210.

McCraw, T. F. (1965). The half-time of cesium-137 in man. Radiol. Health Data 6:711718.

Melo, D. R.; Lipsztein, J. L.; Oliveira, C. A.; Lundgren, D. L.; Muggenburg, B. A.; Guilmette, R. A. (1997). A biokinetic model for ${ }^{137}$ Cs. Health Phys. 73:320-332.

Mewhinney, J. A.; Brooks, A. L.; McClellan, R. O. Comparison of the retention and distribution of injected ${ }^{252} \mathrm{Cf}$ in rats and chinese hamsters. Health Phys. 22:695-700; 1972. 
Mewhinney, J. A.; Diel, J. H. Retention of inhaled ${ }^{238} \mathrm{PuO}_{2}$ in beagles: A mechanistic approach to description. Health Phys. 45: 39-60; 1983.

Mewhinney, J. A.; Ziemer, P. L.; Landolt, R. R. Retention and distribution of injected californium-252 in the rat. Health Phys. 21:860-862; 1971.

Morin, M.; Nenot, J. C.; Lafuma, J. (1974). Distribution and removal by DTPA of injected ${ }^{252} \mathrm{Cf}$ in the rat. Health Phys. 26:323-326.

NCRP (1997). Deposition, Retention and Dosimetry of Inhaled Radioactive Substances, NCRP Report No. 125 (National Council on Radiation Protection and Measurements, Bethesda, MD).

NCRP (1998). Evaluating the reliability of biokinetic and dosimetric models and parameters used to assess individual doses for risk assessment purposes, NCRP Commentary No. 15 (National Council on Radiation Protection and Measurements, Bethesda, MD).

Neuman, W. F. (1964). Blood-bone exchange. In: Bone biodynamics, ed. by H. M. Frost. Boston: Little, Brown, and Co; 393-408.

Newton, D.; Eagle, M. C. (1972). The measurement of californium-252 in vivo. Health Phys. 23:818-825.

Newton, D.; Harrison, G. E.; Kang, C.; Warner, A. J. (1991). Metabolism of injected barium in six healthy men. Health Phys. 61:191-201.

Newton, D.; Harrison, G. E.; Rundo, J.; Kang, C.; Warner, A. J. (1990). Metabolism of $\mathrm{Ca}$ and $\mathrm{Sr}$ in late adult life. Health Phys. 59:433-442.

Norrdin, R. W.; Arnold J. S. (1980). Comparison of ${ }^{47}$ Ca accumulation in samples of trabecular and cortical bone and its retention in ribs, humerus, and femur. In: Bone Histomorphometry, edited by W. S. S. Jee and A. M. Parfitt; pp. 499-500 (abstract).

NRC-CEC (1997). Probabilistic Accident Consequence Uncertainty Analysis. Late Health Effects Uncertainty Assessment, NUREG/CR-6555; EUR 16774; SAND97-2322 (U.S. Nuclear Regulatory Commission, Washington, DC; Office for Publications of the European Communities, Luxembourg).

NRC-CEC (1998). Probabilistic Accident Consequence Uncertainty Analysis. Uncertainty Assessment for Internal Dosimetry, NUREG/CR-6571; EUR 16773; SAND98-0119 (U.S. Nuclear Regulatory Commission, Washington, DC; Office for Publications of the European Communities, Luxembourg). 
Parker, H. G.; de Low-Beer, A.; Isaac, E. L. (1962). Comparison of retention and organ distribution of Am-241 and Cf-252 in mice: The effect of in vivo DTPA chelation. Health Phys. 8:679-684.

Phang, J. M.; Berman, M.; Finerman, G. A.; Neer, R. M.; Rosenberg, L. E; Hahn, T. J. (1969). Dietary Perturbation of calcium metabolism in normal man: compartmental analysis. J. Clin. Invest. 48:67-77.

Poda, G. A.; Hall, R. M. (1975). Two californium-252 inhalation cases. Health Phys. 29:407-409; 1975.

Pollack, S., George, J.N., Reba, R.C., Kaufman, R.M. and Crosby, W.H. (1965) The absorption of non-ferrous metals in iron deficiency. J. Clin. Invest. 44:1470-1473.

Riggs, B. L.; Marshall, J. H.; Jowsey, J.; Heaney, R. P.; Bassingthwaighte, J. B. (1971). Quantitative Ca-45 autoradiography of human bone. J. Lab. Clin. Med. 78:585-598.

Rundo, J.; Sedlet, J. (1973). Retention and elimination of berkelium-249-californium-249 following acute accidental inhalation. In: Proceedings of the 3rd International Congress of the International Radiation Protection Association. Washington, D.C. September 9-14, 1973. IRPA CONF-730907-P1.

Runkle, G. E.; Snipes, M. B.; McClellan, R. O.; Cuddihy, R. G. (1980). Metabolism and dosimetry of inhaled ${ }^{106} \mathrm{RuO}_{4}$ in Fischer-344 rats. Health Phys. 39:543-553.

Sabau MN, Lanzi LH, Rozenfeld M. (1980) Californium-252 dosimetry in phantoms of various dimensions. Radiology. 1980 Dec;137(3):789-93.

Schade, S.G., Felsher, B.F., Bernier, G.M. and Conrad, M.E. (1970a) Interrelationship of cobalt and iron absorption. J. Lab. Clin. Med. 75:435-441.

Schade, S.G., Felsher, B.F., Glade, B.E. and Conrad, M.E. (1970b) Effect of cobalt upon iron absorption. Proc. Soc. Exp. Biol. Med. 75:741-743.

Schanker, L. S.; Mitchell, E. W.; Brown, R. A., Jr. (1986). Species comparison of drug absorption from the lung after aerosol inhalation or intratracheal injection. Drug Metab. Dispos. 14:79-88.

Schlenker, R. A.; Keane, A. T.; Holtzman, R. B. (1982). The retention of Ra-226 in human soft tissue and bone; implications for the ICRP 20 alkaline earth model. Health Phys. 42:671-693.

Schubert, J.; Brodsky, A.; Tyler, S. (1967). The log-normal distribution function as a stochastic model of the distribution of strontium- 90 and other fission products in humans. Health Phys. 13:1187-1204. 
Schulert, A. R.; Peets, E. A.; Laszlo, D.; Spencer, H.; Charles, M.; Samachson, J. (1959). Comparative metabolism of strontium and calcium in man. Int. J. Appl. Radiat. Isotop. 4:144-153.

Schwarz, G.; Dunning, D. E. (1982). Imprecision in estimates of dose from ingested ${ }^{137} \mathrm{Cs}$ due to variability in human biological characteristics. Health Phys. 43:631-645.

Shagina, N. B.; Tolstykh, E. I.; Zalyapin, V. I.; Degteva, M. O.; Kozheurov, V. P.; Tokareva, E. E.; Anspaugh, L. R.; Napier, B. A. (2003). Evaluation of age and gender dependencies of the rate of strontium elimination 25-45 years after intake: Analysis of data from residents living along the Techa River. Radiat. Res. 159:239-246.

Smith T., Edmonds C. J., Barnaby C. F. (1972). Absorption and retention of cobalt in man by whole-body counting. Health Phys. 22:359-367.

Smith T.; Thompson, B. D.; Barnaby C. F. (1971). Measurement of ${ }^{60}$ Co organ burdens in rats and their use in calculations of equilibrium dose rates to various organs of man. Health Phys. 20:195-204.

Smith, V. H. (1972). Therapeutic removal of internally deposited transuranium elements. Health Phys. 22:765-778; 1972.

Spencer, H.; Li, M.; Samachson, J.; laszlo, D. (1960). Metabolism of Strontium-85 and Calcium-45 in Man. Metabolism; 9:916-925.

Stara, J. F.; Nelson, N. S.; Della Rosa, R. J.; Bustad, L. K. (1971). Comparative metabolism of radionuclides in mammals: a review. Health Phys. 20:113-137.

Stark, G. (1968). Studies on synthetic hydroxyapatite crystals with regard to metabolism of calcium, strontium, barium and radium in bone. I. The discrimination against calcium. Biophysik 5:42-54.

Stather, J. W. (1974). Distribution of P-32, Ca-45, Sr-85 and Ba-133 as a function of age in the mouse skeleton. Health Phys. 26:71-79.

Stevens, W.; Bruenger, F. W. Interaction of ${ }^{249} \mathrm{Cf}$ and ${ }^{252} \mathrm{Cf}$ with constituents of dog and human blood. Health Phys. 22:679-683; 1972.

Stevenson, A. F. G. (1975). The influence of age and sex on the activity ratio of yttrium-90 to strontium-90 in the rat skeleton after incorporation of strontium-90. Health Phys. 29:285-290; 1975.

Taylor, D. M. (1959). Metabolism of some trace metals in animals and man. PhD Thesis, University of London; 1959. 
Taylor, D. M.; Lehmann, M.; Planas-Bohne, F.; Seidel, A. The metabolism of radiohafnium in rats and hamsters: A possible analog of plutonium for metabolic studies. Radiat. Res. 95:339-358; 1983.

Taylor, G. N.; Jee, W. S. S.; Mays, C. W.; Dell, R. B.; Williams, J. L.; Shabetari, L. Microscopic distribution of californium-249 and berkelium-249 in the soft tissues of beagles. Health Phys. 22:691-693; 1972.

Thomas, R. G.; Furchner, J. E.; London, J. E.; Drake, G. A.; Wilson, J. S.; Richmond, C R. (1976). Comparative metabolism of radionuclides in mammals--X. Retention of tracer-level cobalt in the mouse, rat, monkey and dog. Health Phys. 31:323-333.

Thompson, R. C.; Weeks, M. H.; Hollis, L.; Ballou, J. F.; Oakley, W. D. (1958). Metabolism of radioruthenium in the rat. Am. J. Roentgen. 79:1026-1044.

Thornberg, C.; Mattsson, S. (2000). Increased ${ }^{137}$ Cs metabolism during pregnancy. Health Phys. 78:502-506.

Valberg, L.S., Ludwig, J. and Olatunbosun, D. (1969) Alteration in cobalt absorption in patients with disorders of iron metabolism. Gastroenterology 56:241-251.

Valberg, L.S., Sorbie, J., Corbett, W.E.N. and Ludwig, J. (1972) Cobalt test for the detection of iron deficiency anaemia. Ann. Int. Med. 77:181-187.

Valentine, T. E. (2001). Evaluation of prompt gamma rays for use in simulating nuclear safeguard measurements. Ann. Nucl. Energy 28:191-201.

Veronese, I.; Giussani, A.; Cantone, M.C.; De Bartolo, D.; Roth, P.; Werner, E. (2001). Kinetics of systemic ruthenium in human blood using a stable tracer. J. Radiol. Prot. 21:31-38.

Wasserman, R. H.; Wardock, A. R.; Comar, C. L. (1959). Metabolic dissociation of short-lived barium-137m from its cesium-137 parent. Science 129:568-569.

Weber, D. A.; Eckerman, K. F.; Dillman, L. T.; Ryman, J. C. (1989). MIRD:

Radionuclide data and decay schemes. Society of Nuclear Medicne, New York, NY.

Wilson, W. B.; Perry, R. T.; Shores, E. F.; Charlton, W. S.; Parish, T. A.; Estes, G. P.; Brown, T. H.; Arthur, E. D.; Bozoian, M.; England, T. R.; Madland, D. G.; Stewart, J. E. (2002). SOURCES 4C: A code for calculation $(\alpha, n)$, spontaneous fission, and delayed neutron sources and spectra. LA-UR-02-1839.

Wood, Jr., W. R., Sheehan, W. E. (1971). Evaluation of the PUQFUA method of calculating systemic burdens. Am. Ind. Hyg. Assoc. J. 32:58-63. 
Wood, S. K.; Farnham, J. E.; Marshall, J. H. (1970). Ca-45, Ba-133, and Ra-226 in 6-to 10-year-old beagle dogs: A 100-day study. In: Radiological Physics Division annual report, Center for Human Radiobiology, July 1969 through June 1970; Argonne, IL: Argonne National Laboratory; ANL-7760, Part II, Biology and Medicine; 110-132.

Yamagata, N.; Iwashima, K.; Iinuma, T. A.; Ishihara, T.; Watari, K. (1971). Long-term retention of radioruthenium in man. Health Phys. 21:63.

Yamagata, N.; Iwashima, K.; Iinuma, T. A.; Watari, K.; Nagai, T. (1969). Uptake and retention experiments of radioruthenium in man - I. Health Phys. 16:159-166.

Yamaguchi, Y. (1994). Age-dependence effective doses for external photons. Radiat. Prot. Dosim. 55(2):123-129.

Zankl, M. ; Drexler, G. ; Petoussi-Hen $\beta$, N. ; Saito, K. (1997). The calculation of dose from external photon exposures using reference human phantoms and Monte Carlo Methods : Part VII : Organ dose due to parallel and environment exposure geometries. GSF-Report 8/97.

Zankl, M.; Fill, U.; Petoussi-Henss, N; Regulla, D. (2002). Organ dose conversion coefficients for external photon irradiation of male and female voxel models. Phys. Med. Biol. 47:2367-2385.

Zapletal, A.; Samanek, M; Paul, T. (1987). Lung function in children and adolescents, methods, reference values (Karger, Basel, Switzerland).

Zundel, W. S.; Tyler, F. H.; Mays, C. W.; Lloyd, R. D.; Wagner, W. W.; Pendleton R. C. (1969). Short half-times of caesium-137 in pregnant women. Nature 221:89-90. 